\title{
ENERGY LABORATORY
}

MASTER

MASSACHUSETTS INSTITUTE OF TECHNOLOGY

\section{DO NOT MICROFILM COVER}

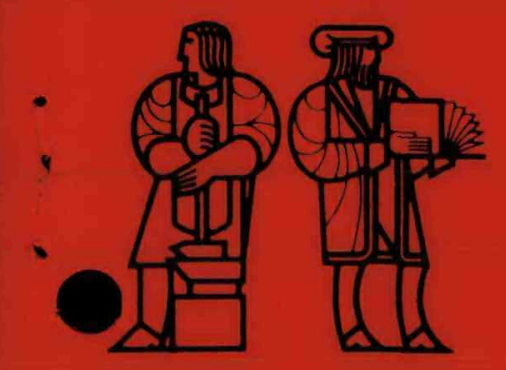




\section{DISCLAIMER}

This report was prepared as an account of work sponsored by an agency of the United States Government. Neither the United States Government nor any agency Thereof, nor any of their employees, makes any warranty, express or implied, or assumes any legal liability or responsibility for the accuracy, completeness, or usefulness of any information, apparatus, product, or process disclosed, or represents that its use would not infringe privately owned rights. Reference herein to any specific commercial product, process, or service by trade name, trademark, manufacturer, or otherwise does not necessarily constitute or imply its endorsement, recommendation, or favoring by the United States Government or any agency thereof. The views and opinions of authors expressed herein do not necessarily state or reflect those of the United States Government or any agency thereof. 


\section{DISCLAIMER}

Portions of this document may be illegible in electronic image products. Images are produced from the best available original document. 


$$
M I T-E L--81-030
$$

DE82 018221

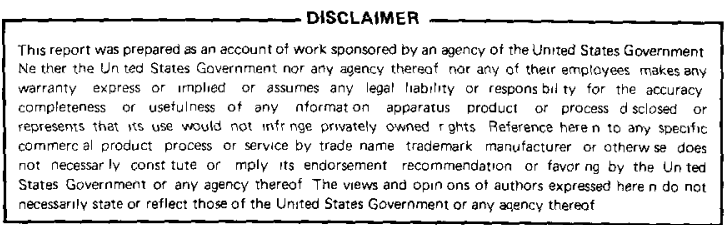

9500636

DEVELOPMENT OF MODELS FOR THE TWO-DIMENSIONAL, TWO-FLUID CODE FOR SODIUM BOILING NATOF-2D

$$
\text { by }
$$

R. G. Zielinski and M. S. Kazimi Energy Laboratory Report No. MIT-EL 81-030

September 1981

$$
\text { AMO1-76EIO2295 }
$$




$$
\begin{gathered}
\text { Energy Laboratory } \\
\text { and } \\
\text { Department of Nuclear Engineering }
\end{gathered}
$$

Massachusetts Institute of Technology

Cambridge, Mass. 02139

\author{
DEVELOPMENT OF MODELS FOR THE \\ TWO-DIMENSIONAL, TWO-FLUID CODE FOR \\ SODIUM BOILING NATOF-2D
}

by

R. G. Zielinski and M. S. Kazimi

September 1981

Topical Report of the MIT Sodtum Bolling Project

sponsored by

U. S. Department of Energy, General Electric Co. and

Hanford Engineering Development Laboratory

Energy Laboratory Report No. MIT-EL 80-030 
REPORTS IN REACTOR THERMAL HYDRAULICS RELATED TO THE MIT ENERGY LABORATORY ELECTRIC POWER PROGRAM

A. Topical Reports (For availability check Energy Labóratory Headquarters, Headquarters, Room E19-439, MIT, Cambridge, Massachusetts 02139)

A.1 General Applications

A.2 PWR Applications

A.3 BWR Applications

A.4 LMFBR Applications

A.l J.E. Kelly, J. Loomis, L. Wolf, "LWR Core Thermal-Hydraulic Analysis-Assessment and Comparison of the Range of Applicability of the Codes COBRA-IIIC/MIT and COBRA-IV-1," MIT Energy Laboratory Report No. MIT-EL-78-026, September 1978.

M. S. Kazimi and M. Massoud, "A Condensed Review of Nuclear Reactor Thermal-Hydraulic Computer Codes for Two-Phase Flow Analys is," MIT Energy Laboratory Report No. MIT-EL-79-018, February 1979.

J.E. Kelly and M.S. Kazimi, "Development and Testing of the Three Dimensional. Two-Fluid Code THERMIT for LWR Core and Subchannel Applications," MIT Energy Laboratory Report No. MIT-EL-79-046.

J.N. Loomis and W.D. Hinkle, "Reactor Core Therma1-Hydraulic Analysis-Improvement and Application of the Code COBRA-IIIC/MIT," MIT Energy Laboratory Report No. MIT-EL-80-027, September 1980.

D.P. Griggs, A.F. Henry and M.S. Kazimi, "Development of a ThreeDimensional Two-Fluid Code with Transient Neutronic Feedback for LWR Applications," MIT Energy Laboratory No. MIT-EL-81-013, Apri1 1981.

J.E. Kelly, S.P. Kao and M.S. Kazimj, "THERMIT-2: A Two-Fluid Model for Light Water Reactor Subchannel Transient Analysis, "MIT Energy Laboratory Report No. MIT-EL-81-014, April 1981.

H.C. No and 11.S. Kazimi, "The Effect of Virtual Mass on the Characteristics and the Numerical Stability in Two-Phase Flows," MIT Energy Laboratory Report No. MIT-EL-81-023, April 1981.

J.W. Jackson and N.E. Todreas, "COBRA IIIC/HIT-2: A Digital Computer Program for Steady State and Transient Thermal-Hydraulic Analys is of Pod Bundle Nuclear Fuel Elements," MiT-EL-81-018, June 1981.

J.E. Kelly, S.P. Kao, and II.S. Kazimi, "User's Guide for THERI!IT-2: A Version of THERIIIT for both Core-llide and Subchannel Analys is of Light Water Reactors," MIT Energy Laboratory Report iVo. I'IT-EL 8i-029, August 1981. 
A.2 P. Moreno; C. Chiu, R. Bowring, E. Khan, J. Liu, and N. Todreas, "Methods for Steady-State Thermal/Hydraulic Analysis of PIV Cores," MIT Energy Laboratory Report No. MIT-EL 76-006, Rev. 1, July 1977, (Orig. 3/77).

J. Liu and N. Todreas, "Transient. Thermal Analys is of PWR's by a Single Pass Procedure Using a Simplified Model Layout, "MIT Energy Laboratory Report No. MIT-EL 77-008, Final, Febiruary 1979 (Draft, June 1977).

J. Liu and N. Todreas; "The Comparison of Available Data on PWR Assembly Thermal Behavior with Analytic Predictions," MIT Energy Laboratory Report ivo. MIT-EL 77-009, Final February 1979, (Draft, June 1977).

A.3 L. Guillebaud, A. Levin, W. Boyd, A. Faya, and L. Wolf, "WOSUB-A Subchannel Code for Steady-State and Transient Thermal-Hydraulic Analys is of Boiling Water Reactor Fuel Bundles," Vol. II, Users Manual, MIT-EL 78-024, JuTy 1977.

L. Wolf; A. Faya, A. Levin, W. Boyd, L. Guillebaud, "WOSUB-A Subchannel Code for Steady State and Transient Thermal-Hydraulic Analys is of Boiling Water Reactor. Fuel Pin Bundles," Vol. III, Assessment and Comparison, MIT-EL 78-025, October 1977.

L. Wolf, A. Faya, A. Levin, L. Guillebaud, "WOSUB-A Subchannel Code for Steady-State Reactor Fuel Pin Bundles," Vol. I, Model Description, MIT-EL 78-023, September 1978.

A. Faya L. Wolf and N. Todreas, "Development of a Method for BWR Subchannel Analys is," MIT-EL 79-027, November 1979.

A. Faya, L. Wolf and N. Todreas, "CANAL User's Manual," MIT Energy Laboratory No. MIT-EL 79-028, November 1979.

A.4 W.D. Hinkle, "Water Tests for Determining Post-Voiding Behavior in the LMFBR," MIT Energy Laboratory Report MIT-EL-76-005, June 1976.

W.D. Hinkle, Ed., "LMFBR Safety and Sodium Boiling - A State of the Art Report," Draft DOE Report, June 1978.

M.R. Granziera, P. Griffith, W.D. Hinkle, M.S. Kazimi, A. Levin, M. Manahan, A. Schor, N. Todreas, G. Wilson, "Development of Computer Code for Multi-dimensional Analys is of Sodium Voiding in the LMFBR," Preliminary Draft Report, July 1979.

M. Granziera, P. Griffith, W.D. Hinkle, M.S. Kazimi, A. Levin, M. Manahan, A. Schor, N. Todreas, R. Vilim, G. Hilson,- "Development of Computer Code Models for Analys is of Subassembly Voiding in the LliFBR," Interim Report of the MTI Sodium Boiling Project Covering Work Through September 30, 1979, liIT-EL-80-005. 
$-i i i-$

A. Levin and P. Griffith, "Development of a Model to Predict Flow Oscillations in Low-Flow Sodium Boiling, "MIT-EL-80-006, April 1980.

M. R. Granziera and M. S. Kazimi, "A Two-Dimensional, Two-Fluid Model for Sodium Boiling in LMFBR Assemblies," MIT- EL-80-011, May 1980.

G. Wilson and M. Kazimi, "Development of Models for the Sodium Version of the Two-Phase Three Dimensional Thermal Hydraulics Code THERMIT," MIT-EL-80-010, May 1980.

R.G. Zielinski and M.S. Kazimi, "Development of Models for the Two-Dimensional, Two-Fluid Code for Sodium Boiling NATOF-2D," MIT Energy Laboratory Report No. MIT-EL-81-030, September 1981. 
B. Papers

B.1 General Applications

B.2 PWR Applications

B.3 BWR Applications

B.4 LMFBR Applications

B.1 J.E. Kelly and M.S. Kazimi, "Development of the Two-Fluid MultiDimensional Code THERMIT for LWR Analys is," Heat Transfer-Orlando 1980, AIChE Symposium Series 199, Vol. 76, August 1980.

J.E. Kelly and M.S. Kazimi, "THERMIT, A Three-Dimensional, Two-Fluid Code for LWR Transient Analys is," Transactions of American Nuclear Society, 34, p. 893, June 1980.

B.2 P. Moreno, J. Kiu, E. Khan, N. Todreas, "Steady State Thermal Analys is of PWR's by a Single Pass Procedure Using a Simplified Method,". American Nuclear Society Transactions, Vol. 26.

P. Moreno, J. Liu, E. Khan, N. Todreas, "Steady-State Thermal Analys is of PWR's by a Single Pass Procedure Using a Simplified Nodal Layout," Nuclear Engineering and Design, Vol. 47, 1978, pp. 35-48.

C. Chiu, P. Moreno, R. Bowring, N. Todreas, "Enthalpy Transfer between PWR Fuel Assemblies in Analys is by the Lumped Subchannel Model," Nuclear Engineering and Design, Vol. 53, 1979, 165-186.

B.3 L. Wolf and A. Faya, "A BWR Subchannel Code with Drift Flux and Vapor Diffusion Transport," American Nuclear Society Transactions, Vol. 28, 1978, p. 553.

S.P. Kao and M.S. Kazimi, "CHF Predictions In Rod Bundles," Trans. ANS, 35, 766 June 1981 .

B.4 W.D. Hinkle, (MIT), P.M. Tschamper (GE), M.H. Fontana (ORNL), R.E. Henry (ANL), and A. Padilla (HEDL), for U.S. Department of Energy, "LMFBR Safety \& Sodium Boiling," paper presented at the ENS/ANS International Topical Meeting on Nuclear Reactor Safety, October 16-19, 1978, Brussels, Belgium.

M.I. Autruffe, G.J. Wilson, B. Stewart and M. Kazimi, "A Proposed Komentum Exchange Coefficient for Two--Phase Modeling of Sodium Boiling," Proc. Int. Meeting Fast Reactor Safety Technology, Vol. 4, 2512-2521, Seattle, Washington, August 1979.

M.R. Granziera and M.S. Kazimi, "NATOF-2D: A Two Dimensional Two-Fluid liodel for Sodium Flow Transient Analys is," Trans. Alis, 33, 515, liovember 1979. 


\author{
DEVELOPMENT OF MODELS FOR THE \\ TWO-DIMENSIONAL, TWO-FLUID CODE FOR \\ SODIUM BOILING NATOF-2D
}

\title{
ABSTRACT
}

Several features were incorporated into NATOF-2D, a twodimensional, two fluid code developed at M.I.T. for the purpose of analysis of sodium boiling transients under LMFBR conditions. They include improved interfacial mass, momentum and energy exchange rate models, and a cell-to-cell radial heat conduction mechanism which was calibrated by simulation of Westinghouse Blanket Heat Transfer Test Program Runs 544 and 545. Finally, a direct method of pressure field solution was implemented into NATOF-2D, replacing the iterative technique previously available, and resulted in substantially reduced computational costs.

The models incorporated into NATOF-2D were tested by running the code to simulate the results of the THORS Bundle $6 \mathrm{~A}$ Experiments performed at Oak Ridge National Laboratory, and four tests from the W-I SLSF Experiment performed by the Hanford Engineering Development Laboratory. The results demonstrate the increased accuracy provided by the inclusion of these effects. 


\section{NOTICE}

This report was prepared as an account of work sponsored by the United States Government and two of its subcontractors. Neither the United States nor the United States Department of Energy, nor any of their employees, nor any of their contractors, subcontractors, or their employees, makes any warranty, express or implied, or assumes any legal liability or responsibility for the accuracy, completeness or usefulness of any information, apparatus, product or process disclosed, or represents that its use would not infringe privately owned rights. 


\section{ACKNOWLEDGEMENTS}

Funding for this project was provided by the United States Department of Energy. This support was deeply appreciated.

A very special thanks is due to Andrei L. Schor, whose enthusiasm for Sodium Bolling provided a constant source of information and inspiration.

The work described in this report was performed primarily by the principal author, Robert G. Zielinski, who submitted this work in partial fulfillment for the M:S. degree in Nuclear Engineering at M.I.T. 
Table of Contents

Page

TITLE PAGE • • . . . . . . . . . . . . . . . . . I

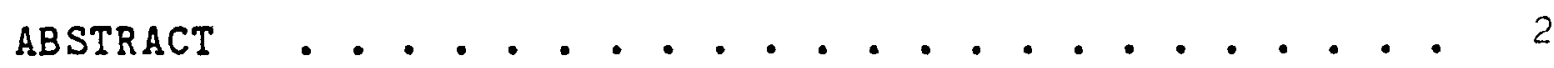

ACKNOWLEDGEMENTS • • • • • • . • • • • • • . . . . 3

TABLE OF CONTENTS . . . . . . . . . . . . . . . . 4

List of Figures . . . . . . . . . . . . . . . 8

List of Tables . . . . . . . . . . . . . . . . 12

Nomenclature . . . . . . . . . . . . . . 13

Chapter 1: INTRODUCTION . . . . . . . . . . . . 17

1.1 Description of the Code . . . . . . . . . 17

1.2 Scope of Work . . . . . . . . . . . 21

1.2.1 Interfacial Mass, Energy,

and Momentum Exchange Models . . . . 21

1.2.2 Fluid Conduction Model . . . . . . 22

1.2.3 Direct Solution of the Pressure Field. 22

1.2.4 Comparison to Experiments on Boiling

Behavior .......... . 23

Chapter 2: INTERFACIAL MASS, ENERGY AND MOMENTUM
EXCHANGE MODELS . - . • • • • • . . . . 24

2.1 Introduction .............. 24

2.2 Conservation Equations Used in NATOF-2D . . . 26

2.3 Interfacial Mass Exchange . . . . . . . 29

2.4 Energy Exchange Rate . . . . . . . . . 40

2.5 Interfacial Momentume Transfer . . . . . . 47

2.6 Programming Information . . . . . . . . 54

Chapter 3: FLUID CONDUCTION MODEL . . . . . . . . . . .

3.1 Introduction . . . . . . . . . . . 55 
Page

3.2 Formulation . . . . . . . . . 57

3.3 Intercell Areas ............ 61

3.4 Implementation Form .......... 65

3.5 Experimental Calibration ......... 68

3.6 A Comparison with Effective Conduction

Mixing Lengths............ 79

3.7 Programming Information . . . . . . . 87

Chapter 4: DIRECT SOLUTION OF THE PRESSURE FIELD . . . 88

4.1 Introduction . . . . . . . . . 88

4.2 Direct Method Solution Techniques . . . . 92

4.3 A Comparison of Direct and Iterative
Methods in NATOF-2D . . . . . . 97

4.4 Programming Information ......... 101

Chapter 5: EXPERIENCES WITH NATOF-2D . . . . . 102

5.1 Introduction . . . . . . . . . 102

5.2 Double versus Single Precision... . . . 103

5.3 On the Modelling of Sodium Reactors . . . 106

5.4 The Mass Exchange Rate ......... 109

5.5 Varying Mesh Spacing . . . . . . . . 113 Chapter 6: VERIFICATION OF MODELS . . . . . . . 115

6.1 Introduction . . . . . . . . . . II5

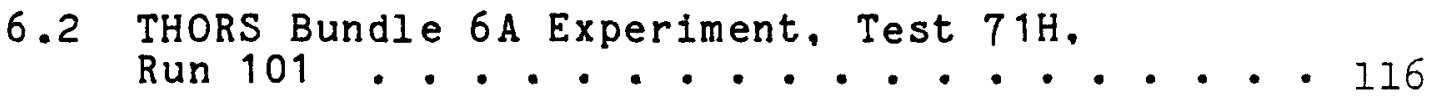

6.2.1 Description of the THORS Bundle

6A Experiment........... 116

6.2.2 Simulation Results . . . . . . . 117 
Page

6.3 The W-1 SLSF Experiment . . . . . . . . 126

6.3.1 Test Objective . . . . . . . . 126

6.3.2 Test Apparatus and Procedure . . . . 127

6.3.3 Tests Chosen for Simulation . . . . . 127

6.4 W-1 SLSF Simulation Results . . . . . . . 133

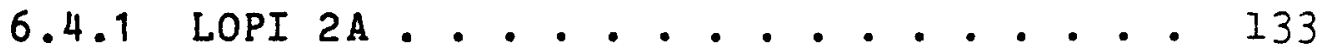

6.4 .2 LOPI 4 . . . . . . . . . . 133

6.4 .3 BWT 2'.............. 138

6.4 .4 BWT 7B'............ 145

Chapter 7: CONCLUSIONS AND RECOMMENDATIONS • . • • • . 151

7.1 Conclusions . . . . . . . . . . . 151

7.2 Recommendations . . . . . . . . . . . 153

References . . . . . . . . . . . . . . . 155

Appendix A: SPECIFIED INLET VELOCITY AND MASS FLOW RATE 157

A.1 Introduction . . . . . . . . . . 157

A.2 Treatment of the Momentum Equations . . . . 159

A.3 Inlet Velocity Boundary Condition . . . . . 165

A. 4 Specified Inlet Mass Flow Rate Boundary
Condition . . . . . . . . . . 167

A.5 Programming Information . . . . . . . 173

Appendix B: NATOF-2D INPUT DESCRIPTION . . . . . . 174

Appendix C: INPUT FILES FOR NATOF-2D TEST CASES . • . 179

C.1 Westinghouse Blanket Heat Transfer Test

Program Run 544 . . . . . . . . . . . . 179

C.2 Westinghouse Blanket Heat Transfer Test

Program Run 545 ............... 180

C.3 THORS Bundle 6A Test $71 \mathrm{H}$ Run 101 . . . . . . 181 
Page

C. 4 W-1 SLSF LOPI 2A ............ 184

C.5 W-1 SLSF LOPI 4 . . . . . . . . . . 186

C.6 W-1 SLSF BWT $2^{\prime}$............. 188

C.7 W-1 SLSF BWT 7B' ............. . 191

AppendiX D: NATOF-2D HEXCAN MODEL ......... 194

Appendix E: NATOF-2D SPACER PRESSURE DROP MODEL . . . 196

Appendix F: SAMPLE OUTPUT . . . . . . ... 198

Appendix G: LISTING OF NEW NATOF-2D SUBROUTINES . . . 202 
List of Figures

Number

Page

1.1 Typical Arrangement of Cells Used in NATOF-2D 19

2.1 Condensation Coefficient as a Function of Pressure... . . . . . . . 33

2.2 A Comparison of New and Old Mass Exchange Rates for a Superheat of $2{ }^{\mathrm{C}}$..... 38

2.3 A Comparison of New and Old Mass Exchange Rates. for a Superheat of $20^{\circ} \mathrm{C} . . .39$

2.4 Variations between Vapor and Saturation Temperatures for an Interfacial Heat Transfer Nusselt Number of $0.006 . .445$

2.5 Vapor and Saturation Temperatures for an Interfacial Heat Transfer Nusselt Number

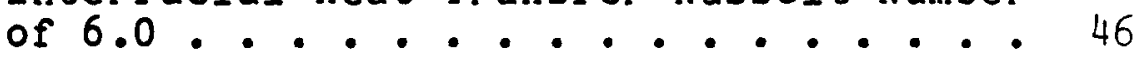

2.6 Values of $\Gamma$ and $K$ as a Function of Void Fraction for Typical Operating Conditions.......... 51

3.1 Top View of Fluid Channels Showing the Radial Heat Transfer Between Them..... . 58

3.2 Top View of Fluid Channels Showing Radial Cell Boundary Numbering Scheme. . . 62

3.3 Unit Cell used in NATOF-2D . . . . . . 63

3.4 Radial Temperature Profile at the End of the Heated Zone for Various Effective Nusselt Numbers ........ . 71

3.5 A Comparison Between Westinghouse Run 544 and NATOF-2D Radial Temperature Profiles at the Heated Zone Midplane for $\mathrm{Nu}_{I}=22$ and $\mathrm{Nu}_{2}=28$.......... . . . .

3.6 A Comparison Between Westinghouse Run 544 and NATOF-2D Radial Temperature Profiles at the End of the Heated Zone for $\mathrm{Nu}_{1}=22$ and $\mathrm{Nu}_{2}=28$ 
List of Figures (continued)

Number

Page

3.7 A Comparison Between Westinghouse Run 544 and NATOF-2D Radial Temperature Profiles 25 Inches Downstream of Heated Zone for $\mathrm{Nu}_{1}=22$ and $\mathrm{Nu}_{2}=28$...... 74

3.8 Normalized Heat Input per Rod for Westinghouse Blanket Heat Transfer Test Program Run 545 . . . . . . . . . . 75

3.9 A Comparison Between Westinghouse Run 545 and NATOF-2D Radial Temperature Profiles at the Heated Zone Midplane for $\mathrm{Nu}_{1}=22$ and $\mathrm{Nu}_{2}=28$.......... 76

3.10 A Comparison Between Westinghouse Run 545 and NATOF-2D Radial Temperature Profiles at the End of the Heated Zone for $\mathrm{Nu}_{I}=22$ and $\mathrm{Nu}_{2}=28$. . . . . . . . . 77

3.11 A Comparison Between Westinghouse Run 545 and NATOF-2D Radial Temperature Profiles 25 Inches Downstream of the Heated Zone for $\mathrm{Nu}_{1}=22$ and $\mathrm{Nu}_{2}=28$....... 78

3.12 Possible Temperature Distributions Which Yield the Same Cell Averaged Temperature . . 81

3.13 19-Pin Cell Geometry Used for the Calculation of Effective Mixing Lengths .... 83

4.1 Arrangement of Cells for Pressure Field Matrix Shown in Figure 4.2 . . . . 89

4.2 Pressure Field Matrix . . . . . . . 9 90

4.3 Upper Triangular Matrix ......... 93

4.4 Lower Triangular Matrix.......... 93

4.5 A Comparison of Steady State CPU Usage Between the Direct and Iterative Techniques

(10 Axial Levels, 5 Radial Nodes) . . 98

4.6 A Comparison of Transient CPU Usage Between the Direct and Iterative Techniques

(10 Axial Levels, 5 Radial Nodes) . . 99 
List of Figures (continued)

Number

Page

6.1 Location of Cells Used in the NATOF-2D

Simulation of the THORS Bundle $6 \mathrm{~A}$

Experiment .............

6.2 NATOF-2D Predicted Inlet Mass Flow Rate of THORS Bundle $6 \mathrm{~A}$ Test $71 \mathrm{H}$ Run 101 ... 121

6.3 NATOF-2D Temperature Profiles of Central Channel At Various Points in Time (THORS Bundle 6A, Test 71H, Run 101) . . 122

6.4 NATOF-2D Predicted Temperature Histories at End of the Heated Zone for the Central and Edge Channels (THORS Bundle 6A, Test 71H, Run 101) . . 123

6.5 A Comparison of NATOF-2D Predicted Liquid and Vapor and Velocities

(THORS Bundle 6A, Test $71 \mathrm{H}$, Run 101) . . 125

6.6 . Location of Cells Used in the NATOF-2D

Simulation of the W-1 SLSF Experiments . 130

6.7 W-1 SLSF Test LOPI 2A Experiment Inlet Mass Flow Rate / 5, ....... 134

6.8 A Comparison Between Experiment and NATOF-2D

Predicted Temperature Histories at the End of the Heated Zone for the Central Channel (W-1 SLSF Test LOPI 2A) . . . 135

6.9 A Comparison of NATOF-2D Central and Edge Channels Temperature Histories at the End of the Heated $Z$ one

(W-1 SLSF Test LOPI 2A) . . . . . 136

6.10 W-1 SLSF Test LOPI 4 Inlet Mass Flow Rate / 5 / ............ 137

6.11 A Comparison Between W-1 SLSF Test LOPI 4 and NATOF-2D Temperature Histories at the End of the Heated Zone for the Central Channel ............. 139

6.12 A Comparison Between W-1 SLSF Test LOPI 4 and NATOF-2D Temperature Histories at the End of the Heated Zone for the Edge Channel 
List of Figures (continued)

Number

Page

6.13 NATOF-2D Void Maps for W-1 SLSF Test LOPI 4 for the Central and the Middle Channels (Void Fraction $=0.1$ ) ....... I41

6.14 W-1 SLSF Test BWT 2' Inlet Mass Flow Rate.. 142

6.15 A Comparison Between W-1 SLSF Test BWT 2' and NATOF-2D Temperature Histories at the End of the Heated Zone for the Central Channel ......... I I43

6.16 A Comparison Between W-1 SLSF Test BWT 2' and NATOF-2D Temperature Histories at the End of the Heated Zone for the Edge Channel . . . . . . . . . 144

6.17 A Comparison Between W-1 SLSF Test BWT 7B' and NATOF-2D Predicted Inlet Mass Flow

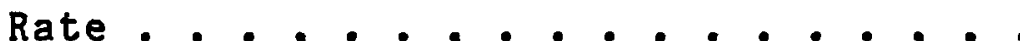

6.18 A Comparison of NATOF-2D Central Channel Void Maps for $C F=1.0$ and $C F=0.01$

(W-1 SLSF Test 7 $\mathrm{B}^{\prime}$ )

6.19 A Comparison of NATOF-2D Edge Channel Void Maps $f$ or $C F=1.0$ and $C F=0.01$

(W-1 SLSF TEst $7 \mathrm{~B}^{\prime}$ )

6.20 A Comparison Between NATOF-2D and W-1 SLSF

BWT 7B' Temperature Histories at the End of the heated Zone for the Central Channel

A.1 Positions Used for the Evaluation of Variables ........... 161

A.2 Example Cell Numbering Scheme for Flow Boundary Calculation...... 168

A.3 Cell Configuration for Matrix Shown in Figure A.4........... 170

A. 4 Pressure Field Matrix With Flow Boundary Calculation 


\section{List of Tables}

Number

Page

2.1 Parameters Used in $\mathrm{K}$ versus $\Gamma$ Comparison . . 52

3.1 Test Parameters for Westinghouse Blanket Heat Transfer Test Program Bundle. . . 69

3.2 Conduction Mixing Length Theory Results . . 84

5.1 Convergence Criteria Versus Minimum Timestep (Single Precision) .......... 104

5.2 A Comparison of PWR and LMFBR Properietes . . 107

5.3 A Comparison of Water and Sodium Properties. 107

6.1 Description of THORS Bundle 6A . . . . . . 118

6.2 Geometric Parameters of $\mathrm{W}-1$ SLSF Test

Bundle ............. 128

6.3 Power and Flow Rates of the W-1 SLSF Experiment . . . . . . . . . . 129 
Nomenclature

Letter

Definition

Units(SI)

a

A

$\underline{\text { A }}$

Ar

Ar*

b

CF

d

D

$D_{C}$

$D_{e}$

f

g

$h_{f g}$

h

$\mathrm{H}$

j

K

$\underline{L}$

$\ell_{i j}$

$\ell_{i j}^{*}$

M
Fuel pellet radius

Area

Matrix of pressure coefficients

Volumetrically averaged

Radial area between cells

Radial area constant

Fuel rod radius

Condensation factor

Wire wrap diameter

Di ameter

Conductive diameter

Equivalent diameter

friction factor

Gravity constant

Enthalpy of vaporization

Enthalpy

Heat transfer coefficient

Mass flux

Momentum exchange coefficient

Lower triangular matrix

Effective conduction mixing length

Centroid-to-centroid distance of adjacent cells

Momentum exchange rate m

$m^{2}$

$m^{2}$

-

m

-

m

m

m

m

$\mathrm{m} / \mathrm{s}^{2}$

$\mathrm{J} / \mathrm{kg}$

$\mathrm{J} / \mathrm{kg}$

$w / m^{2}-a$

$\mathrm{kg} / \mathrm{m}^{2}-\mathrm{s}$

$\mathrm{kg} / \mathrm{m}^{3}-\mathrm{s}$

-

m

$\mathrm{kg} / \mathrm{m}^{2}-\mathrm{s}^{2}$ 


\begin{tabular}{|c|c|c|}
\hline Letter & & Units (SI) \\
\hline M & Molecular weight of particles & $\mathrm{kg} / \mathrm{mole}$ \\
\hline $\mathrm{n}$ & Row number & - \\
\hline $\mathrm{Nu}$ & Nusselt Number & - \\
\hline $\mathbf{P}$ & Pressure & $N / m^{2}$ \\
\hline $\mathrm{P}$ & Fuel pitch & m \\
\hline $\mathrm{P}$ & Perimeter & $\mathrm{m}$ \\
\hline$q$ & Heat flux & $w / m^{2}$ \\
\hline$q \cdot "$ & Power density & $\mathrm{W} / \mathrm{m}^{3}$ \\
\hline$q^{\prime \prime}$ & Heat flux & $w / m^{2}$ \\
\hline$Q$ & Heat generation rate & $\mathrm{w} / \mathrm{m}^{3}$ \\
\hline $\mathbf{r}$ & Radial spacial coordinate & m \\
\hline $\mathbf{R}$ & Universal gas constant & $\mathrm{J} / \mathrm{mole}-\mathrm{gK}$ \\
\hline$s_{1 j}$ & Length of common cell boundary & m \\
\hline SBT & Stable bolling timestep & $\mathbf{s}$ \\
\hline $\mathrm{t}$ & Time & s \\
\hline $\mathrm{T}$ & Temperature & $o_{K}$ \\
\hline U & Velocity & $\mathrm{m} / \mathrm{s}$ \\
\hline$\underline{U}$ & Upper triangular matrix & - \\
\hline $\mathrm{v}$ & Volume & m \\
\hline w & Bandwidth of matrix & - \\
\hline W & Total inlet mass flow rate & $\mathrm{kg} / \mathrm{s}$ \\
\hline $\mathbf{z}$ & Axial spacial coordinate & m \\
\hline
\end{tabular}




\begin{tabular}{clc}
$\begin{array}{l}\text { Nomenclature (continued) } \\
\text { Greek }\end{array}$ & \multicolumn{1}{c}{ Definition } & Units(SI) \\
$\alpha$ & Vold fraction & $\mathrm{m}^{2} / \mathrm{s}$ \\
$\alpha$ & Thermal diffusivity & - \\
$\delta$ & Increment & - \\
$\Delta$ & Increment, spacing & $\mathrm{kg} / \mathrm{m}^{3}-\mathrm{s}$ \\
$\Gamma$ & Mass exchange rate & - \\
$\eta$ & Interfacial velocity & $\mathrm{kg} / \mathrm{m}^{3}$ \\
$\rho$ & Density & - \\
$\sigma$ & Mass exchange coefficient &
\end{tabular}

Subscripts

$\begin{array}{ll}r & \text { Radial position } \\ v & \text { Axial position } \\ e & \text { Vapor } \\ i & \text { Liquid } \\ \text { e } & \text { Interface } \\ c & \text { Evaporation } \\ \text { s } & \text { Condensation } \\ \text { T } & \text { Saturation } \\ \text { int } & \text { Total } \\ c & \text { Interface } \\ \text { w } & \text { Conduction }\end{array}$


Nomenclature (continued)

Superscripts
$n+1$
New time step
n
old time step 


\section{Chapter 1}

INTRODUCTION

1.1 Description of the Code

The computer code NATOF-2D was developed at the Massachusetts Institute of Technology for the simulation of both steady state and transient conditions in Liquid Metal Fast Breeder Reactors / $1 /$. The code uses the two fluid model of conservation equations, and a two-dimensional r-z geometry which takes advantage of the symmetry found in LMFBR bundles. The two dimensional nature of the calculation allows the multidimensional effects of sodium bolling to be observed, without the corresponding high computational costs of a three dimensional code.

The model treats the liquid and vapor phases separately, coupled by only the exchange coefficients. No assumption is made about the relationship between the properties of the two phases, which allows greater generality. The method thus requires the solution of the mass, momentum and energy conservation equations for each phase.

For calculational purposes, the fuel assembly is divided into a finite number of axial and radial mess cells. There is no constraint as to the positioning or number of axial levels other than at each level the mesh spacing 
remains constant. However, the boundaries between cells in the radial direction must fall between the fuel pin centerlines, and so the number of radial cells is limited to the number of fuel pin rows. Figure 1.1 shows a typical arrangement of cells used by NATOF-2D.

The fluid properties of a cell are treated as the volumetric average of the properties in that cell, which necessitates the use of sufficiently small cells in order to obtain detailed information. The fluid velocities are evaluated at the faces of the cell, and are assumed to be uniform across each cell face. The unknowns of the calculation are $P, \alpha, T_{v}, T_{\ell}, U_{v z}, U_{v r}, U_{\ell z}$, and $U_{\ell r}$.

NATOF-2D uses a partially implicit method to solve the fluid dynamics equations. The terms involving sonic velocity and interfacial exchange are treated implicitly. However, for the convective terms, only the velocities are treated implicitly, while all other factors are evaluated at the previous timestep. This method imposes a timestep limitation such that

$$
\Delta t \leq \frac{\Delta z}{U_{z}}
$$

In most cases, this is not a detrimental constraint, since this timestep is usually the same order of magnitude as the time at which information is required.

The equations are solved by reduction to a Newton Iteration problem, in which the unknowns become linearized. 
-19-

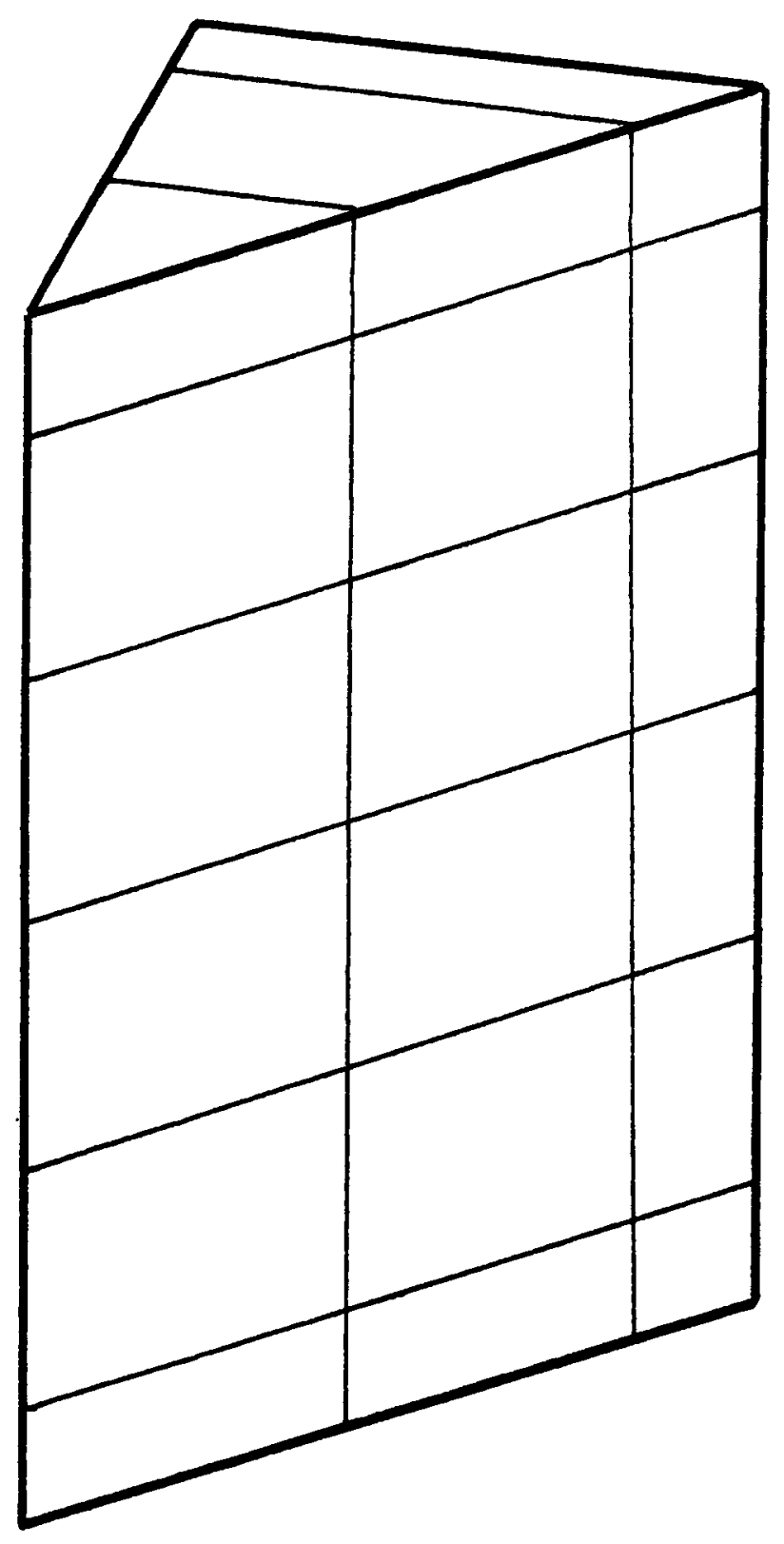

Figure 1.1 Typical Arrangement of Cells Used in 
These equations are further reduced to a set of linear equations involving only the pressures of a cell. The pressure field is then solved for by either an iterative or a direct technique, and all variables are then updated. The advantage of using a Newton Iteration technique is that a solution can always be attained by taking a sufficiently small timestep. The heat conduction equations are solved implicitly and coupled to the fluid dydnamics equations.

The code has the capability to operate with pressure, velocity or flow boundary conditions at the inlet, and a pressure boundary condition at the outlet. The velocity and flow inlet boundary conditions are new features incorporated into NATOF-2D, and are described in Appendix $A$.

NATOF-2D is able to handle the most severe sodium bolling conditions, including flow reversal. The work covered in this thesis addresses some of the major difficulties encountered in past sodium boiling simulations. 
1.2 Scope of Work

1.2.1 Interfacial Mass, Energy and Momentum Exchange Models The constituative equations used for the calculation of the interfacial mass, energy and momentum exchange rates have been improved to more physically account for the observed phenomena. The terms have a pronounced effect on the ability of the two-fluid model to simulate sodium boiling transients, since one of the major assumptions of this work is that for void fractions below 0.957 the vapor phase does not come in contact with the wall. Thus these terms often represent the only source of mass, momentum and energy for the vapor phase.

The mass exchange rate, which has the strongest effect of any constituative relation on the running of the code, has been implemented in a more basic form than before, using the kinetic theory of condensation. It is treated in a fully implicit manner so that all dependencies on the independent variables are accounted for. The momentum exchange rate has been modified to take into account the effects of mass exchange. Finally, the energy exchange rate has been modelled to prevent the appearance of highly subcooled vapor or superheated liquid in two phase flow transients. 
1.2.2 Fluid Conduction Model

The high conductivity of liquid sodium coupled with the turbulence found in LMFBR bundles usually results in small radial temperature gradients across the core. Previously, the only mechanism available in NATOF-2D for the modelling of this phenomenon was energy exchange between cells due solely to mass transfer. However, the small radial velocities allowed large temperature differences to exist between internal channels and the edge channel.

Therefore, a radial heat conduction model has been incorporated into NATOF-2D. The model is applied only when single phase liquid is present in adjacent cells since the conductivity of the vapor phase is very low. Presently, only radial conduction has been employed in the code, since axial convection effects tend to dominate any axial conduction effects.

Calibration of the model is accomplished by simulation of two Westinghouse Blandket Heat Transfer Test Program experiments / 2 /. The model developed is also compared to analytic results based on conduction mixing length theory 131.

1.2.3 Direct Solution of the Pressure Field

The computer time usage of NATOF-2D is strongly dependent on the solution technique used for the calculation of the pressure field. A more efficient method has been 
implemented into NATOF-2D which uses a direct method to solve the pressure field matrix, rather than the iterative technique previously employed. The advantages of this are substantially reduced running time, and the capability of using smaller axial mesh cell spacings.

1.2.4 Comparison to Experiments on Boiling Behavior

The major experiences encountered while running NATOF-2D are documented in Chapter 5 to serve as a foundation for future work, and also provide an explanation for any changes deemed necessary to the previously derived models, especially the mass exchange rate. Also, some of the difficulties with sodium boiling codes in general are discussed.

Chapter 6 discusses the results obtained for five transients performed by NATOF-2D. One test was a simulation of the Thors Bundle $6 \mathrm{~A}$ experiments conducted at Oak Ridge National Laboratory / 4 /, while the other four are from the SLSF $W-1$ experiments done at the Hanford Engineering Development Laboratory / $5 /$.

Finally Chapter 7 summarizes the findings of this thesis, and makes recommendations for future development of NATOF-2D. 
Chapter 2

INTERFACIAL MASS, ENERGY, AND MOMENTUM

EXCHANGE MODELS

\subsection{Introduction}

In the two fluid model NATOF-2D, each phase in the flow field is described by a set of mass, energy, and momentum equations. Each of these equations takes into account the interactions which occur between the phases. This is accomplished by the use of empirical correlations or simple physical models, that describe the mass, energy and momentum exchange rates at the liquid/vapor interface.

One of the requirements of two phase flow modeling is that no mass, energy, or momentum be gained or lost at the interface. This is the so called "jump condition" at the interface. This requirement is met if the conservation equations of each phase can be summed together, and the interface exchange terms cancel each other.

For the sodium boiling transients which NATOF-2D was designed to simulate, these exchange rates take on a special significance. One of the basic assumptions of this work is that only the liquid phase is in contact with the wall for values of void fraction up to 0.957. Thus, for many applications, the vapor phase is entirely dependent on the liquid phase as a mass, energy or momentum source, and 
thereby dependent on the accuracy of the exchange models incorporated into this code.

This chapter will cover the models developed for interfacial transport exchange, and compare the results with those previously used in NATOF-2D / $1 /$. 
2.2 Conservation Equations Used in NATOF-2D

Since this chapter deals with the modelling of the interfacial mass, enegy and momentum exchange rates, the conservation equations in the form used by NATOF-2D are summarized in this section. Since NATOF-2D is a two-phase, two-dimensional $R-Z$ code, for each phase there will be one mass and one energy conservation equation, and two momentum equations (one for each direction) at each node. Given below are the eight conservation equations written in control volume form.

\section{Mass Conservation}

liquid phase:

$$
\begin{gathered}
\frac{\partial}{\partial t} \int_{V}(1-\alpha) \rho_{\ell} d V+\int_{A_{z+}}-\int_{A_{z-}}(1-\alpha) \rho_{\ell} U_{\ell z} d A+\int_{A r+}-\int_{A r-}(1-\alpha) \rho_{\ell} U_{\ell r} d A \\
=-\int_{V} \Gamma d V
\end{gathered}
$$

vapor phase:

$$
\begin{gathered}
\frac{\partial}{\partial t} \int_{\mathrm{V}} \alpha \rho_{\mathrm{V}} d V+\int_{\mathrm{A}_{\mathrm{z}+}}-\int_{\mathrm{A}_{\mathrm{z}-}} \alpha \rho_{\mathrm{V}} \mathrm{U}_{\mathrm{vz}} \mathrm{dA}-\int_{\mathrm{A}_{\mathrm{r}+}}-\int_{\mathrm{A}_{\mathrm{r}-}} \alpha \rho_{\mathrm{V}} \mathrm{U}_{\mathrm{vr}} \mathrm{dA} \\
=\int_{\mathrm{V}} \Gamma \mathrm{dV}
\end{gathered}
$$




\section{Energy Conservation}

\section{liquid phase:}

$$
\begin{aligned}
& \frac{\partial}{\partial t} \int_{V}(1-\alpha) \rho_{\ell}\left(e_{\ell}+U_{\ell}^{2} / 2\right) d V+\int_{A_{z+}}-\int_{A_{z-}}(1-\alpha) \rho_{\ell} U_{\ell}\left(e_{\ell}+U_{\ell}^{2} / 2\right) d A+ \\
& \int_{A_{r+}}-\int_{A_{r-}}(1-\alpha) \rho_{\ell} U_{\ell r}\left(e_{\ell}+U_{\ell}^{2} / 2\right) d A=\int_{V} Q_{\ell} d V-\int_{V}(1-\alpha) \rho_{\ell} g U_{\ell z} d V- \\
& \int_{A_{W}} U_{\ell} \cdot f_{\ell} d A+\int_{A_{\ell}} P \cdot n \cdot U_{\ell} d A+\int_{V} P \frac{\partial \alpha}{\partial t} d V-\int_{A_{i}} q_{\ell i} d A \\
& \frac{\partial}{\partial t} \int_{V} \alpha \rho_{V}\left(e_{V}+U_{V}^{2} / 2\right) d V+\int_{A_{Z}+}-\int_{A_{Z-}} \alpha \rho_{V} U_{v z}\left(e_{V}+U_{V}^{2} / 2\right) d A+ \\
& \int_{A_{r+}}-\int_{A_{-}} \alpha \rho_{v} U_{v r}\left(e_{v}+U_{v}^{2} / 2\right) d A=\int_{V} Q_{v} d V-\int_{V} \alpha \rho_{v} g U_{v z} d V- \\
& \int_{A_{W}} U_{V} \cdot f_{V} d A+\int_{A_{V}} p \cdot n \cdot U_{V} d A-\int_{V} p \frac{\partial \alpha}{\partial t} d V+\int_{A_{1}} q_{v i} d A
\end{aligned}
$$

liquid phase

$$
\begin{aligned}
& \frac{\partial}{\partial t} \int_{V}(1-\alpha) \rho_{\ell} U_{\ell z} d V+\int_{A_{z+}}-\int_{A_{z-}}(I-\alpha) \rho_{\ell} U_{\ell z}^{2} d A+ \\
& \int_{A_{r+}}-\int_{A_{r-}}(1-\alpha) \rho_{\ell} U_{\ell z} U_{\ell r} d A-\oint_{A_{\ell}} P \cdot \hat{k} \cdot \hat{n} d A=-\int_{A_{W}} f_{\ell z} d A- \\
& \int_{V}(1-\alpha) \rho_{\ell} g d V-\int_{V} M_{\ell z} d V
\end{aligned}
$$


$-28-$

vapor phase

$$
\begin{aligned}
& \frac{\partial}{\partial t} \int_{V} \alpha \rho_{v} U_{v z} d V+\int_{A_{z+}}-\int_{A_{z-}} \alpha \rho_{v} U_{v z}^{2} d A+\int_{A_{r+}}-\int_{A_{r-}} \alpha \rho_{v} U_{v z} U_{v r} d A- \\
& \oint_{A_{V}} P \cdot \hat{k} \cdot \hat{n} d A=-\int_{A_{W}} f_{v z} d A-\int_{V} \alpha \rho_{v} g d V+\int_{V} M v z d V \\
& \text { Momentum Conservation--Radial Direction } \\
& \text { liquid phase } \\
& \frac{\partial}{\partial t} \int_{V}(I-\alpha) \rho_{\ell} U_{\ell r} d V+\int_{A_{z+}}-\int_{A_{z-}}(I-\alpha) \rho_{\ell} U_{\ell r} U_{\ell z} d A+ \\
& \int_{A_{r+}}-\int_{A_{r-}}(I-\alpha) p_{\ell} U_{\ell r}^{2} d A-\oint_{A_{\ell}} P \cdot \hat{r} \cdot \hat{n} d A= \\
& -\int_{A_{W}} f_{\ell r} d A+\int_{V} M_{\ell r} d V \\
& \text { vapor phase } \\
& \frac{\partial}{\partial t} \int_{V} \alpha \rho_{v} U_{v r} d V+\int_{A+}-\int_{A_{-}} \alpha \rho_{v} U_{v z} U_{v r} d A+\int_{A_{r+}}-\int_{A_{-}} \alpha \rho_{v} U_{v r}^{2} d A \\
& -\oint_{A_{V}} P \cdot \hat{r} \cdot \hat{n} d A=\int_{A_{W}} f_{v r} d A-\int_{V} M_{v r} d V
\end{aligned}
$$

$(2.8)$ 


\subsection{Interfacial Mass Exchange}

In the mass conservation equations for the liquid and vapor phase (equations 2.1 and 2.2) $\Gamma$ represents the mass exchange rate between phases, and will be defined as positive for evaporation. $\Gamma$ has units of $\mathrm{kg} / \mathrm{m}^{3}-\mathrm{s}$. At the present time, the accepted model for the mass exchange rate is based on the kinetic theory of condensation. This model views the interaction simply as the difference between a flux of particles arriving at the interface, and a flux of particles departing from the interface. The particles are assumed to be arriving from the vapor phase, and departing from the liquid phase. When the arrival rate exceeds the departure rate, condensation is occurring. In the reverse situation, evaporation takes place and when the net flux is zero, an equilibrium condition exists. The derivation of the mass exchange rate is essentially due to Schrage 161.

Using a Maxwell-Boltzmann distribution, it is possible to show that in a stationary container the mass flux of particles passing in either direction through the interface is given by:

$$
j_{i}=\left(\frac{M}{2 \pi R}\right)^{\frac{1}{2}} \frac{P}{T} \frac{1}{2}
$$

where

$$
\begin{aligned}
& j_{i}=\text { mass flux of phase } i\left(\mathrm{~kg} / \mathrm{m}^{2}-\mathrm{s}\right) \\
& M=\text { molecular weight of particles }
\end{aligned}
$$




$$
\begin{aligned}
& R=\text { universal gas constant } \\
& P=\text { pressure exerted by the particles } \\
& T=\text { temperature of the particles }
\end{aligned}
$$

If there exists a progress velocity on the vapor side towards the interface such that $j_{V}=\rho_{v} v_{p}$ then

$$
j_{V}=\left(\frac{M}{2 \pi R}\right)^{\frac{1}{2}} \frac{P}{T} \frac{1}{2} \Psi .
$$

where

$$
\begin{gathered}
\Psi=e^{-\phi^{2}}+\phi \pi(1+\operatorname{erf} \phi) \\
\phi=\frac{\mathrm{V}_{\mathrm{p}}}{(2 \mathrm{RT} / \mathrm{M})^{\frac{1}{2}}}=\frac{j}{\rho_{\mathrm{v}}(2 \mathrm{RT} / \mathrm{M})^{\frac{1}{2}}} \\
\mathrm{~V}_{\mathrm{p}}=\text { progress velocity }
\end{gathered}
$$

At the liquid-vapor interface not all the molecules striking the surface will condense. Therefore, $\sigma_{C}$ is defined as the fraction of molecules striking the surface which actually do condense. In a similar manner, $\sigma_{e}$ represents the ratio of the flux of molecules actually leaving the interface to the flux given by equation 2.9.

At the condensing surface, molecules are arriving at a progress flow rate $\rho_{\mathrm{V}} \mathrm{p}$, and molecules are departing the surface at a rate equivalent to that of molecules in a 
stationary container. Thus the net flux towards the surface is given by:

$$
\begin{aligned}
& j=j_{V}-j_{\ell} \\
& j=\left(\frac{M}{2 \pi R}\right)^{\frac{1}{2}}\left(\sigma_{c} \Psi \frac{P_{V}}{T_{V}} \frac{1}{2}-\sigma_{e} \frac{P_{\ell}}{T_{\ell}} \frac{1}{2}\right)
\end{aligned}
$$

If it is assume that $\phi \ll 1$, or in other words that the condensation rate is low, $\Psi$ can be approximated by the following expression:

$$
\psi=\sqrt{\pi}\left(\frac{j}{\rho_{\mathrm{V}}\left(2 \mathrm{RT}_{\mathrm{V}} / \mathrm{M}\right)^{\frac{1}{2}}}\right)+1
$$

Substituting this into equation 2.13 yields

$$
f=\frac{2}{2-\sigma_{c}}\left(\frac{M}{2 \pi R}\right)^{\frac{1}{2}}\left(\sigma_{c} \frac{P_{V}}{T_{V}} \frac{1}{2}-\sigma_{e} \frac{P_{\ell}}{T_{\ell}} \frac{1}{2}\right)
$$

When the two phases are in equilibrium, the net flux, $j$, is equal to zero, and $\sigma_{c}=\sigma_{e}$. Since the values of the individual coefficients in non-equilibrium systems have not been determined, it is justified to set $\sigma_{c}=\sigma_{e}=\sigma$. Using this approximation the net flux becomes:

$$
j=\frac{2 \sigma}{2-\sigma}\left(\frac{M}{2 \pi R}\right)^{\frac{1}{2}}\left(\frac{P_{V}}{T_{V}} \frac{1}{2}-\frac{P_{\ell}}{T_{\ell}} \frac{1}{2}\right)
$$

and the mass exchange rate is thus

$$
\Gamma=-j A=A \frac{2 \sigma}{2-\sigma}\left(\frac{M}{2 \pi R}\right)^{\frac{1}{2}}\left(\frac{P_{\ell}}{T_{\ell}} \frac{1}{2}-\frac{P_{V}}{T_{V}} \frac{1}{2}\right)
$$

where

$$
A=\text { the interfacial area per unit volume }
$$


The literature shows a wide variation in the value of $\sigma$ for sodium, ranging from $\sigma=1.0$ at low pressures to $\sigma=0.001$ at atmospheric pressures (See figure 2.1). Rohsenow / 7 /, however, attributes this variation to the presence of non-condensible gases which tend to congregate at the interface. These gases add an additional resistance to condensation. Tests conducted on nearly gas-free systems where the flow was high show that any gases present are swept away from the interface, and $\sigma=1.0$ for all pressures.

In the models developed for NATOF-2D, it is assumed that only the liquid phase is in contact with the wall for values of void fraction up to $\alpha_{\text {dryout }}$ Below this value, all heat gains to the vapor phase are solely from the liquid phase. When the liquid phase is evaporating, the vapor phase is entering the system at the saturation temperature. Similarly, condensation occurs when the liquid phases loses heat to the wall, and becomes subcooled. The vapor phase again condenses at the saturation temperature. Thus, for $\alpha<\alpha_{\text {dryout, }}$ it is justified to set $T_{V}$ to $T_{S}$ and $P_{V}$ to $P_{S}$ in equation 2.17 .

For values of $\alpha>\alpha_{\text {dryout, }}$ the liquid becomes entrained in the vapor phase, and then it is the vapor which experiences the heat losses and gains. Thus for this case, $T_{\ell}=T_{S}$, and $P_{\ell}=P_{S}$ in equation 2.17. In order to obtain 


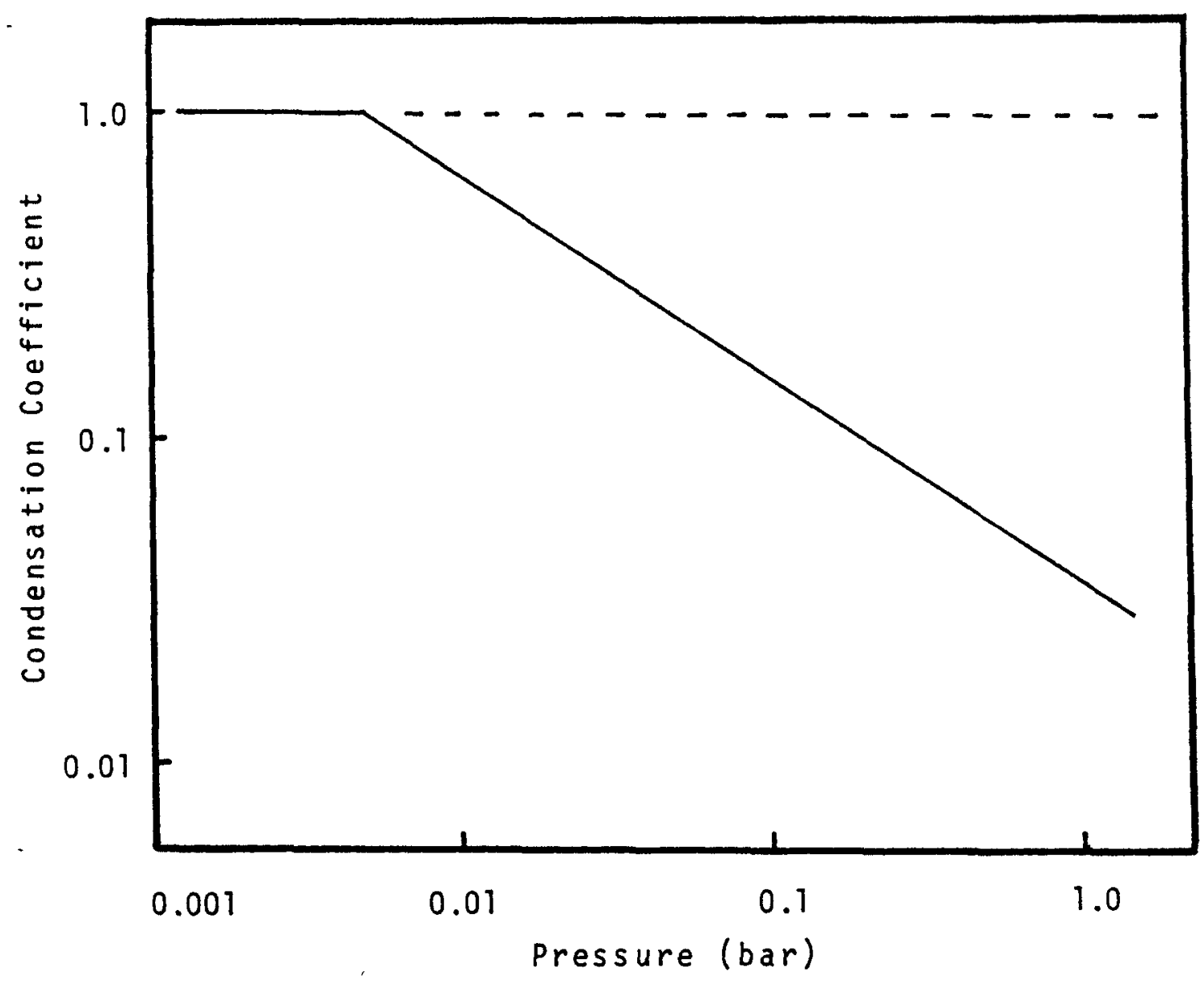

Figure 2.1 Condensation Coefficient as a Function of Pressure 
the correct behavior of this relation, it is necessary to reverse the sign of equation 2.17 so that in a superheated vapor environment, the entrained liquid evaporates, instead of condensing. Equation 2.30 of the next section confirms this behavior.

For the range of temperatures in which sodium boiling and condensation occurs, $\int_{l}^{\frac{1}{2}} \simeq \mathrm{T}_{\mathrm{S}}^{\frac{1}{2}}$ and $\mathrm{T}_{\mathrm{V}}^{\frac{1}{2}} \simeq \mathrm{T}_{\mathrm{S}}^{\frac{1}{2}}$. With this approximation, the final form of the mass exchange rate is arrived at.

$\alpha<\alpha_{\text {dryout }}$

$$
\Gamma=A^{n+1} \frac{2 \sigma}{2-\sigma}\left(\frac{M}{2 \pi R}\right)^{\frac{1}{2}}\left(\frac{P_{\ell}-P_{S}}{T_{S}^{\frac{1}{2}}}\right)^{n+1}
$$

$\alpha>\alpha_{\text {dryout }}$

$$
\Gamma=A^{n+1} \frac{2 \sigma}{2-\sigma}\left(\frac{M}{2 \pi R}\right)^{\frac{2}{2}}\left(\frac{P_{v}-P_{S}}{T_{S}^{\frac{1}{2}}}\right)^{n+1}
$$

where

$$
\begin{aligned}
\mathrm{P}_{\mathrm{V}}= & \text { pressure corresponding to a saturation } \\
& \text { temperature of } \mathrm{T}_{\mathrm{V}} \\
\mathrm{P}_{\ell}= & \text { pressure corresponding to a saturation } \\
& \text { temperature of } \mathrm{T}_{\ell} \\
\mathrm{P}_{\mathrm{S}}= & \text { system pressure } \\
\mathrm{T}_{\mathrm{S}}= & \text { saturation temperature } \\
\mathrm{A}= & \text { interfacial area calculated implicitly }
\end{aligned}
$$

The formulations previously used for the mass exchange rate in NATOF-2D were: 
For evaporation

$$
\Gamma=\sigma A^{n} \alpha^{n}(1-\alpha)^{n+1} \sqrt{\frac{R}{2 \pi M}}\left(\frac{\rho_{v}{ }^{2} h_{f g}}{P_{S}}\right)^{n}\left(\frac{T_{\ell}-T_{s}}{T_{S}^{\frac{1}{2}}}\right)^{n+I}
$$

For condensation

$$
\Gamma=\sigma A^{n} \alpha^{n+1}(1-\alpha)^{n} \sqrt{\frac{R}{2 \pi M}}\left(\frac{\rho_{v}{ }^{2} h_{f g}}{P_{S}}\right)^{n}\left(\frac{T_{v}-T_{s}}{T_{S}^{\frac{l}{2}}}\right)^{n+1}
$$

These relations were based on the assumption that $\Delta T / T_{S} \ll 1$, where $\Delta T=T_{\ell}-T_{S}$. Simulations by NATOF-2D, however, show that $\Delta T$ can be quite large. These results will be discussed in further detail in Chapter 5. The interfacial areas in equations 2.20 and 2.21 were calculated explicitly, and the term $\alpha(1-\alpha)$ was added to force $\Gamma$ to go to zero for single phase flow. The present formulation treats all terms implicitly, including the areas, and has eliminated the $\alpha(1-\alpha)$ term. The areas are from wilson $/ 8 /$, and depend on the flow regime. The following is a summary of the equations used.

$$
\begin{aligned}
& \alpha<\alpha_{\mathrm{m}} \\
& \mathrm{A}_{I}=\frac{3 \alpha}{r_{\mathrm{m}}} \\
& \alpha_{\mathrm{m}}=\frac{2}{3}\left(\frac{\mathrm{r}_{\mathrm{m}}^{2}}{\mathrm{D}}\right) \frac{\pi}{\sqrt{3}(\mathrm{P} / \mathrm{D})^{2}-\pi / 2} \\
& \mathrm{r}_{\mathrm{m}}=6 . \times 10^{-4}
\end{aligned}
$$


$\alpha_{m}<\alpha<0.55$

$$
A_{2}=\frac{4}{D} \sqrt{\frac{3 \pi \alpha}{2 \sqrt{3}(P / D)^{2}-\pi}}
$$

$0.55<\alpha<0.65$

$$
A_{3}=a+b \cdot \bar{\alpha}+c \cdot \bar{\alpha}^{2}+a \cdot \bar{\alpha}^{3}
$$

where

$$
\begin{aligned}
& \bar{\alpha}=\frac{\alpha-0.55}{0.65-0.55 \quad a=A_{2}} \quad b=\frac{\partial A_{2}}{\partial \alpha} 2 \\
& c=3\left(A_{4}-A_{3}\right)-\frac{\partial A_{4}}{\partial \alpha}-2 \cdot \frac{\partial A_{2}}{\partial \alpha} \\
& d=\frac{\partial A_{4}}{\partial \alpha}+\frac{\partial A_{2}}{\partial \alpha}+2\left(A_{2}-A_{4}\right)
\end{aligned}
$$

$0.65<\alpha<0.957$

$$
A_{4}=\frac{4}{D} \sqrt{\frac{2 \sqrt{3 \pi(P / D)^{2}}\left(2 \sqrt{\left.3(P / D)^{2}-\pi\right)^{2}}-\frac{\pi \alpha}{2 \sqrt{3(P / D)^{2}}-\pi}\right.}{}}
$$

$$
0.957<\alpha<1.0
$$

$$
A_{5}=A_{4} \cdot \sqrt{\frac{1-\alpha}{1-0.957}}
$$


A transition regime area, $A_{3}$, which is a polynomial fit between $A_{2}$ and $A_{4}$, has been added in order to keep the areas and their derivatives with respect to $\alpha$ continuous. A comparison was made between the previous and present mass exchange rate formulations. The system pressure used for this comparison was 2 bars, and the results are shown in figure 2.2 and 2.3 for liquid superheats of $2^{\circ} \mathrm{C}$ and $20^{\circ} \mathrm{C}$ respectively. The results show that the new formulation predicts a more rapid vapor production especially in high void regions. Even discounting the effects of the $\alpha(1-\alpha)$ term, the present mass exchange rate still is 2 to 4 times greater than the one previously implemented. Thus more vigorous and sustained boiling for the same superheats is expected. 


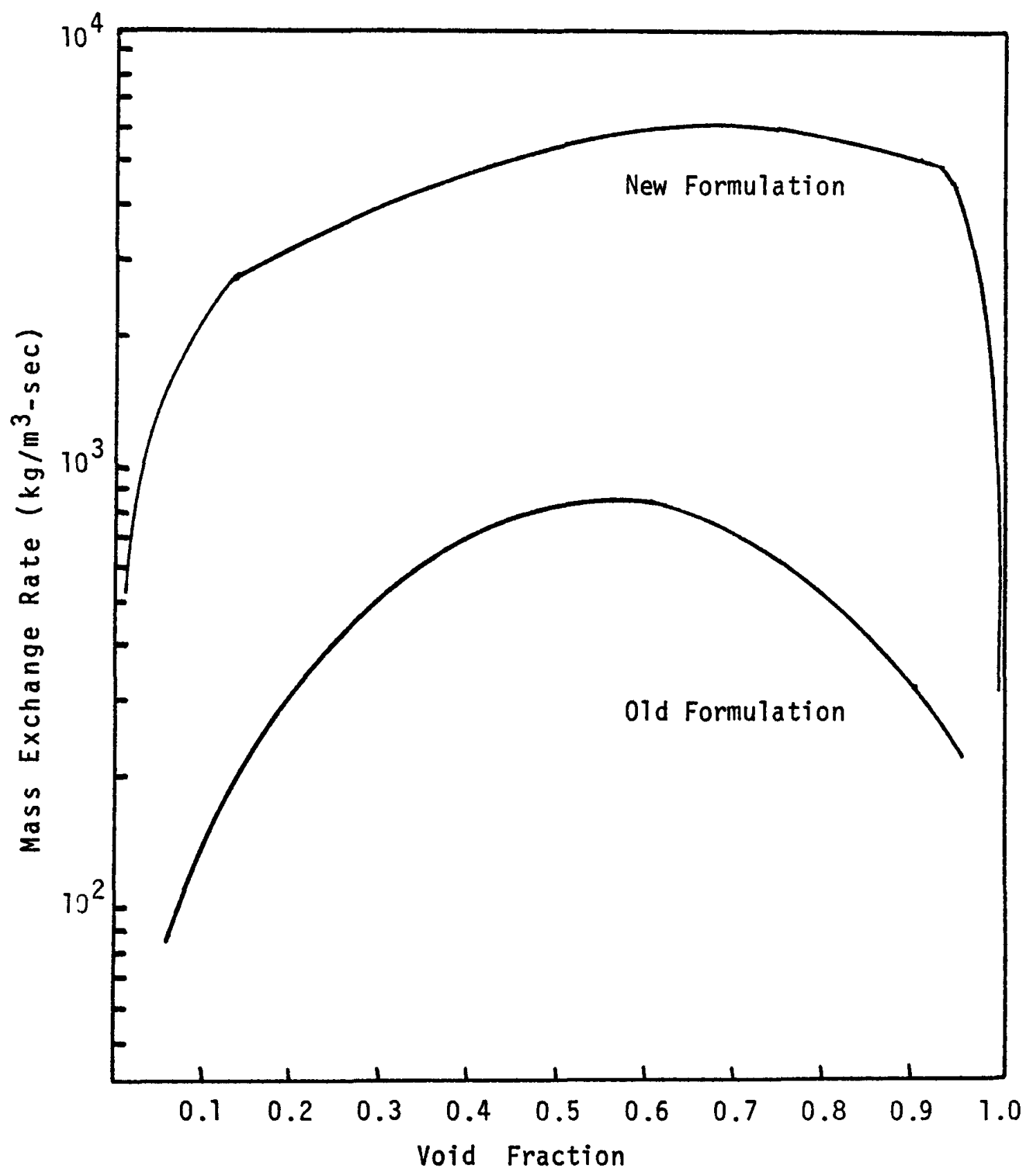

Figure 2.2 A Comparison of New and $01 \mathrm{~d}$ Mass Exchange Rates for a Superheat of $2^{\circ} \mathrm{C}$ 


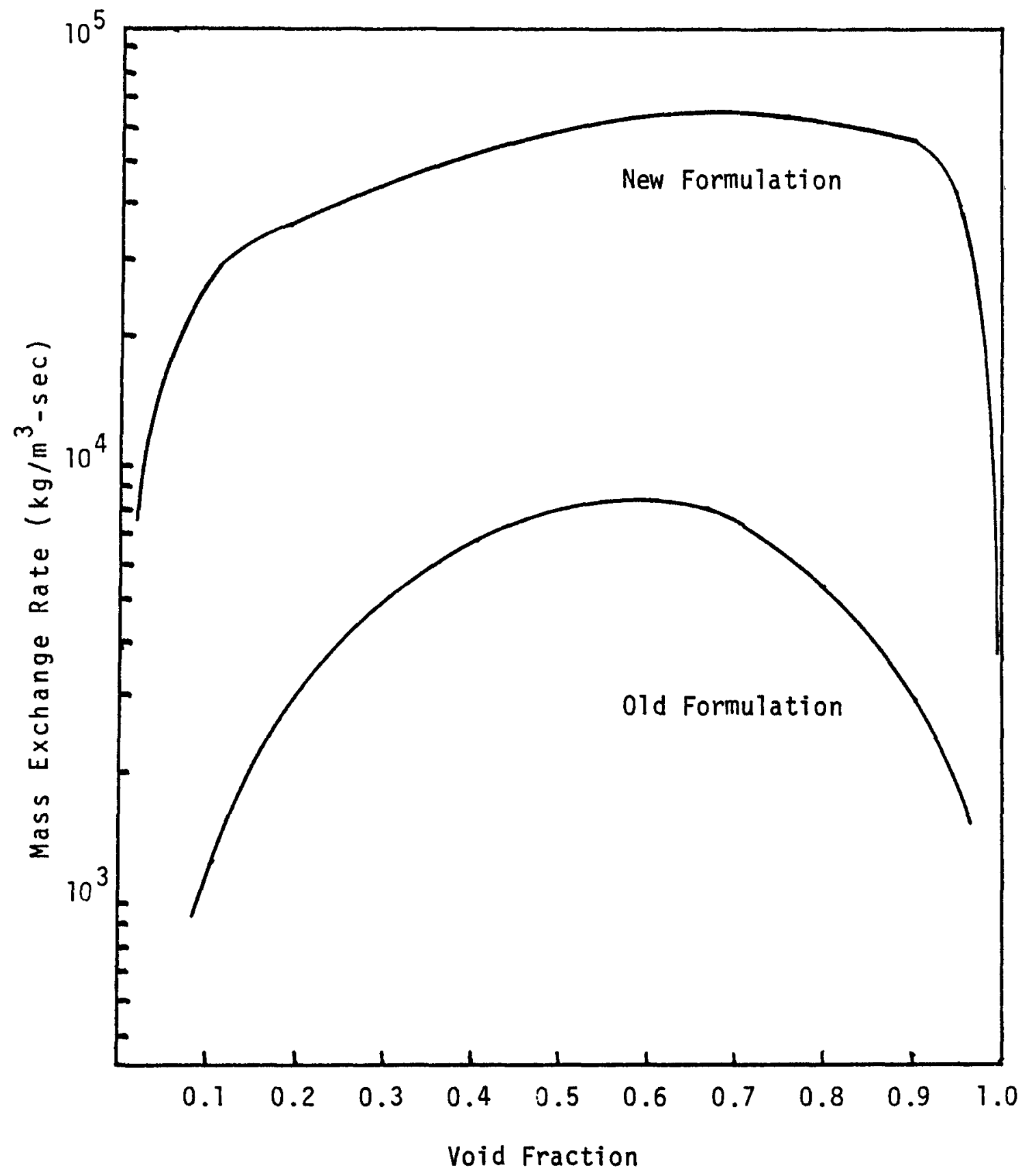

Figure 2.3 A Comparison of New and $01 d$ Mass Exchange Rates for a Superheat of $20^{\circ} \mathrm{C}$ 


\subsection{Energy Exchange Rate}

Reliable constituative relations for interphase heat transfer are not available at the present time. This is due in part to the insufficient attention which this phenomenon has recieved until only recently, and also to the extreme difficulty in gathering useful data on the subject.

Starting with the two phase energy conservation equations, equations 2.3 and 2.4 , one can define an energy exchange due to the difference in temperature between the phase and the interface, and an energy exchange associated with the heat transferred by virtue of mass exchange. With this premise, the energy exchange from the liquid/vapor interface to the vapor become

$$
q_{i v}=\Gamma \cdot h_{v s}+A_{i} H_{i v}\left(T_{1}-T_{v}\right)
$$

Similarly, the energy exchange from the liquid to the liquid/vapor interface is:

$$
q_{\ell I}=\Gamma \cdot h_{\ell s}+A_{i} H_{\ell I}\left(T_{\ell}-T_{i}\right)
$$

where

$$
\begin{aligned}
\Gamma= & \text { mass exhange rate } \\
h_{v s}= & \text { enthalpy of the vapor at the saturation } \\
& \text { temperature } \\
h_{\ell_{S}}= & \text { enthalpy of the liquid at the } \\
& \text { saturation temperature } \\
A_{1}= & \text { interfacial area }
\end{aligned}
$$




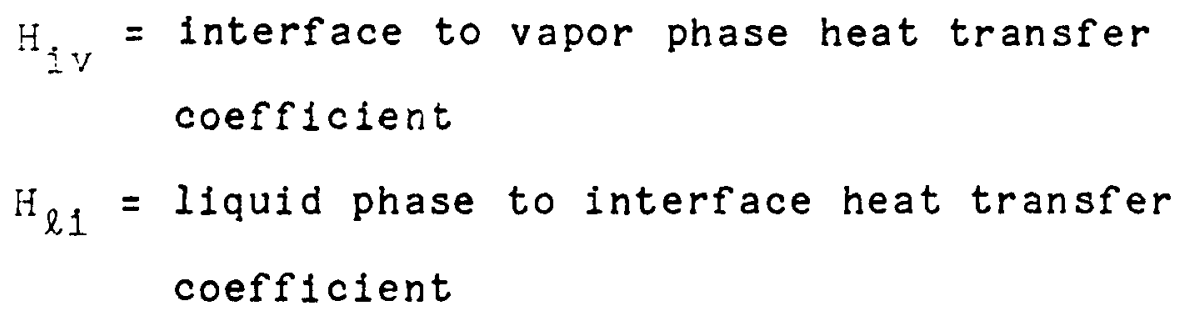

Since the "jump condition" at the interface requires that

$$
q_{i v}=q_{l i}
$$

we have

$$
\Gamma \cdot h_{V S}+A_{i} H_{i v}\left(T_{I}-T_{V}\right)=\Gamma \cdot h_{\ell S}+A_{i} H_{\ell i}\left(T_{\ell}-T_{S}\right)
$$

Equation 2.29 can be used to solve for the mass exchange rate to yield

$$
\Gamma=\frac{H_{i v} A_{i}\left(T_{V}-T_{i}\right)+H_{\ell i} A_{1}\left(T_{\ell}-T_{i}\right)}{h_{f g}}
$$

The above relationship shows that if $\mathrm{H}_{i v}$ and $\mathrm{H}_{\ell 1}$ were known, and if $T_{1}$ was defined, the mass exchange rate would be determined. Unfortunately, there is a lack of data on the interface heat transfer coefficients at the present time. Therefore, an alternative is to use either equation 2.27 or 2.28 and the formulation given in section 2.3 for the interfacial energy exchange rate. One cannot use equation 2.27 for the vapor energy equation and equation 2.28 for the liquid energy equation simultaneously since there would be no guarantee that the jump condition was being satisfied. Attempts to define an interface temperature with a 
value somewhere in the range between the liquid and vapor temperatures have proven fruitless. For an interface temperature based on two infinite bodies in contact, $T_{j}$ is given by the relation

$$
\frac{T_{v}-T_{1}}{T_{1}-T_{\ell}}=\frac{\sqrt{\left(k \rho c_{p}\right)_{\ell}}}{\sqrt{\left(k \rho c_{p}\right)_{v}}}
$$

Since the conductivity and density of the liquid phase is so much greater than that of the vapor phase, solution of equation 2.31 yields $T_{i} \simeq T_{l}$. This result would be acceptable is $T_{\ell}$ stayed near the saturation temperature when both phases are present, but difficulties experienced in attaining a high sodium vapor condensation rate have resulted in vapor coexisting with liquid which is subcooled by as much as $100^{\circ} \mathrm{C}$.

Therefore, the decision was made to set the interfacial temperature to the saturation temperature. The saturation temperature was chosen since it is the equilibrium temperature for a two-phase mixture. As previously stated, for values of $\alpha<\alpha_{\text {dryout }}$, the vapor gains heat solely from the liquid. In an evaporating state the assumption that $T_{1}=T_{s}$ implies that all the liquid superheat is utilized as latent heat for evaporation. And in a condensing state where $T_{\ell}<T_{S}$, the vapor is kept at the saturation temperature, and all heat losses from the vapor are by virtue of mass transfer to the liquid phase. For 
$\alpha>\alpha_{\text {dryout, }}$ the roles of each phase will be reversed. With this understanding, the final form of the interfacial energy exchange rate becomes:

$\alpha<\alpha$ dryout

$$
q_{i}=\Gamma^{n+l_{h s}}+A_{i}^{n+l_{H V}}\left(T_{s}^{n+1}-T_{v}^{n+1}\right)
$$

$\alpha>\alpha$ dryout

$$
q_{i}=\Gamma^{n+I_{h}}{ }_{\ell s} A^{n+1} H_{\ell i}\left(T_{\ell}^{n+1}-T_{s}^{n+1}\right)
$$

where

$$
\begin{aligned}
& \mathrm{H}_{i v}=\mathrm{Nu} \frac{\mathrm{k}_{\ell}}{\mathrm{D}_{e}} \\
& \mathrm{H}_{\ell i}=\mathrm{Nu} \frac{\mathrm{k}_{\mathrm{v}}}{\mathrm{D}_{e}}
\end{aligned}
$$

The previous formulation of the interfacial heat exchange rate was

$$
q_{i}=\Gamma_{e} h_{v s}+\Gamma_{c} h_{\ell s}+A_{i} H_{i}\left(T_{\ell}-T_{v}\right)
$$

This formulation effectively kept $T_{V}$ equal to $T_{l}$, and led to situations of the vapor phase being subcooled by as much as $100^{\circ} \mathrm{C}$. The present formulation has eliminated this problem as is shown in figure 2.5 .

The nusselt number chosen for the interfacial heat transfer coefficients has a pronounced effect on the temperature of the phases. To illustrate this, three 
simulations of a sodium boiling transient were run in which only the interfacial nusselt number was varied. In these cases there was no switch in correlations at $\alpha=\alpha_{\text {dryout }}$. The temperatures given correspond to those found at the top of the heated section of the fuel bundle. As can be seen in figure 2.4, where the vapor and liquid temperatures have been plotted versus time, a small nusselt number $(\mathrm{Nu}=0.006)$ leads to quite a variation between the vapor temperature and the saturation temperature. At approximately 0.55 seconds after bolling inception, at a void fraction corresponding to adryout, the vapor phase began to superheat to high levels. The liquid temperature stayed very close to the saturation temperature.

When $\mathrm{Nu}=6, \mathrm{~T}_{\mathrm{V}} \simeq \mathrm{T}_{\mathrm{S}}$ and $\mathrm{T}_{\ell} \simeq \mathrm{T}_{\mathrm{S}}$ as figure 2.5 indicates. This test case also showed that the saturation temperature was more stable with time, and less prone to wild fluctuations. For $\mathrm{Nu}=6000$, the results were about the same.

Based on these simulations, a value of $\mathrm{Nu}=10$ is recommended. A value in this range will keep the vapor at the saturation temperature, but not make the sensible heat contribution term the dominating one in equations 2.32 and 2.33 


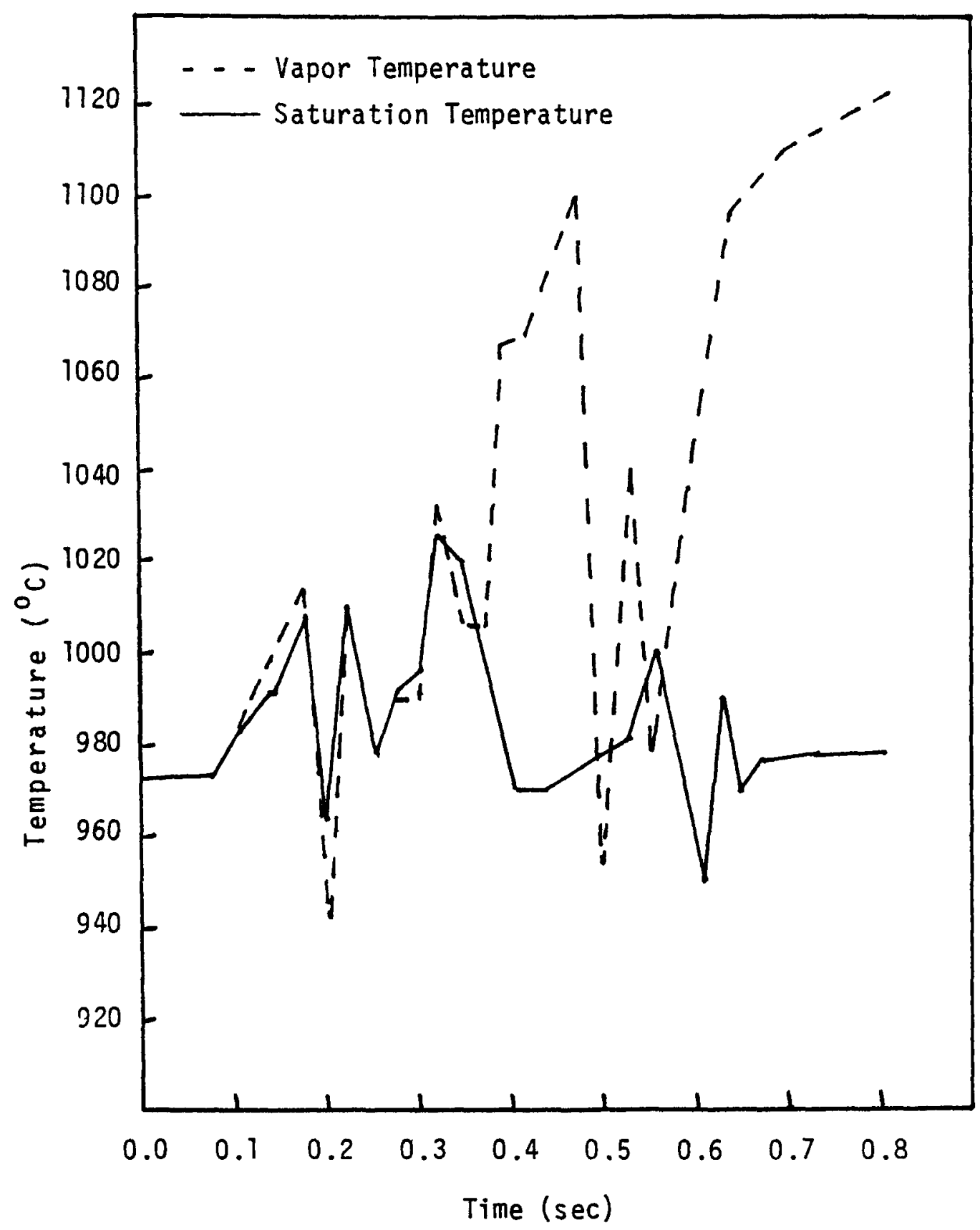

Figure 2.4 Variations Between Vapor and Saturation Temperatures for an Interfacial Heat Transfer Nusselt Number of 0.006 


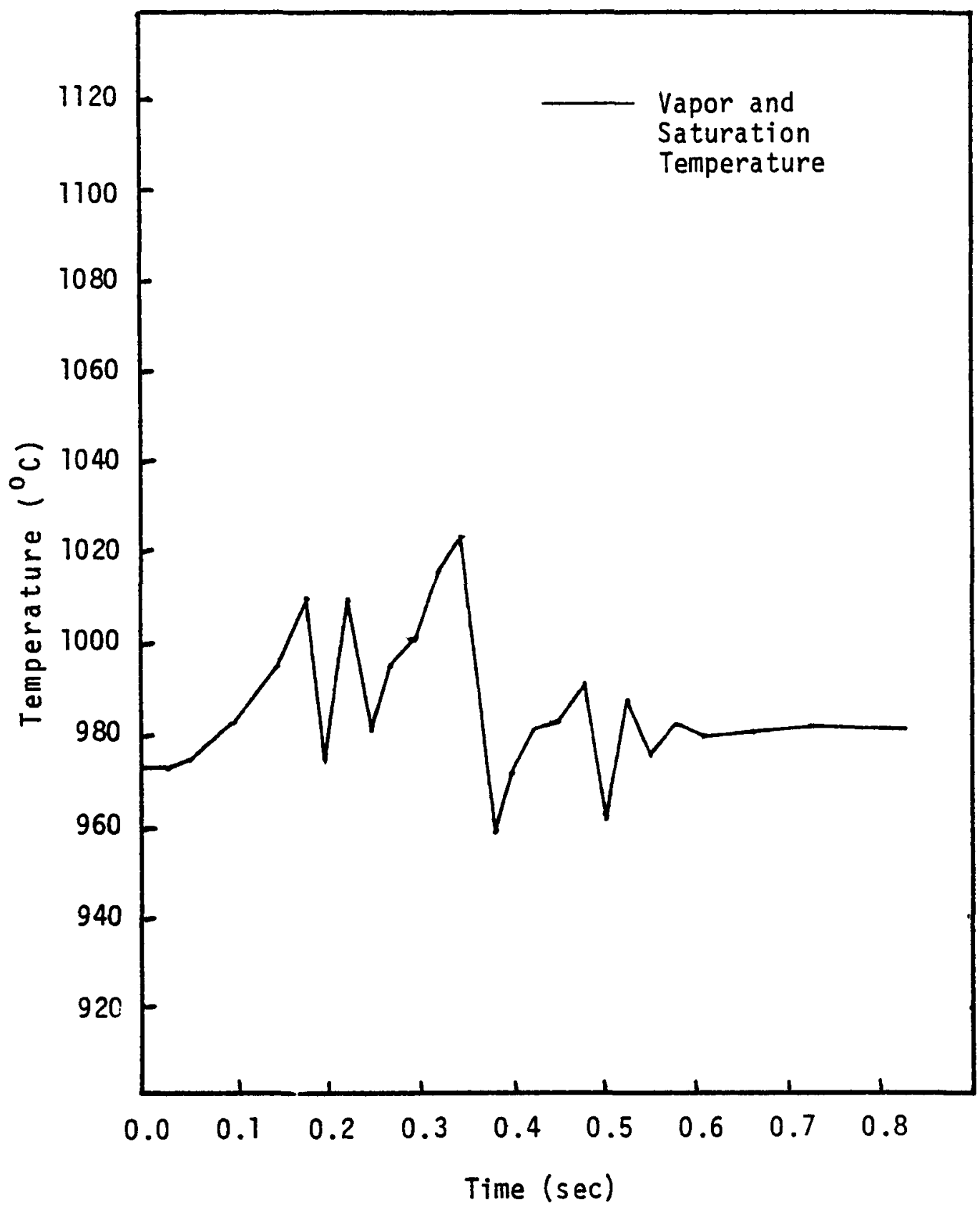

Figure 2.5 Vapor and Saturation Temperatures for an Interfacial Heat Transfer Nusselt Number of 6.0 
2.5 Interfacial Momentum Transfer

The interfacial momentum exchange rate, similar to the interfacial energy exchange rate, is composed of two terms. The first term takes into account the momentum gain due to mass exchange across the interface, and the second term accounts for the effect of shear stresses at the interface. This section will show how these terms can be combined into a single term which contains both of these effects.

The momentum conservation equation for the vapor phase in the z-direction written in differential form is

$$
\begin{aligned}
& \frac{\partial}{\partial t}\left(\alpha \rho_{v} U_{v z}\right)+\frac{\partial}{\partial z}\left(\alpha \rho_{v} U_{v z}^{2}\right)+\frac{\partial}{\partial r}\left(\alpha \rho_{v} U_{v r} U_{v z}\right)+ \\
& \alpha \frac{\partial P}{\partial z}=-f_{W z}-\alpha \rho_{v} g-M_{i z}-U_{i z} \Gamma
\end{aligned}
$$

where

$$
\begin{aligned}
M_{i z}= & \text { shear stress contribution } \\
U_{i z} \Gamma= & \text { contribution due to mass exchange which is } \\
& \text { traveling at an interfacial velocity } \\
& u_{i}=\eta u_{\ell}+(1-\eta) u_{v} \text {, where } \eta \text { is a weighting } \\
& \text { factor }(0 \leq n \leq 1)
\end{aligned}
$$

In order to facilitate the implimentation of the finite difference scheme utilized by NATOF-2D, it is necessary to cast equation 2.35 into non-conservative form. This is accomplished by applying the product rule of differentiation to the following terms: 


$$
\begin{aligned}
& \frac{\partial}{\partial t}\left(\alpha \rho_{v} U_{v z}\right)=\alpha \rho_{v} \overline{\partial U}_{v z}+U_{v z} \frac{\partial}{\partial t}\left(\alpha \rho_{v}\right) \\
& \frac{\partial}{\partial z}\left(\alpha \rho_{V} U_{V z}^{2}\right)=\alpha \rho_{V} U_{V z} \frac{\partial \bar{U}_{v z}}{\partial z}+U_{V z} \frac{\partial}{\partial z}\left(\alpha \rho_{V} U_{V z}\right) \\
& \frac{\partial}{\partial r}\left(\alpha \rho_{V} U_{V z} U_{V r}\right)=\alpha \rho_{V} U_{V r} \frac{\partial U_{V z}}{\partial r}+U_{V z} \frac{\partial}{\partial r}\left(\alpha \rho U_{V r}\right)
\end{aligned}
$$

Substituting these values into equation 2.35 , the vapor momentum equation becomes:

$$
\begin{aligned}
& \alpha \rho_{\mathrm{v}} \frac{\partial \mathrm{U}_{\mathrm{v} z}}{\partial t}+\mathrm{U}_{\mathrm{vz}} \frac{\partial}{\partial t}\left(\alpha \rho_{\mathrm{v}}\right)+\alpha \rho_{\mathrm{v}} \mathrm{U}_{\mathrm{vz}} \frac{\partial \mathrm{U}_{\mathrm{vz}}}{\partial t}+\mathrm{U}_{\mathrm{vz}} \frac{\partial}{\partial z}\left(\alpha \rho \mathrm{U}_{\mathrm{vz}}\right)+ \\
& \alpha \rho_{\mathrm{V}} \mathrm{U}_{\mathrm{vr}} \frac{\partial \mathrm{U}_{\mathrm{V}}}{\partial \mathrm{r}}+\mathrm{U}_{\mathrm{vz}} \frac{\partial}{\partial r}\left(\alpha \rho U_{\mathrm{vr}}\right)+\alpha \frac{\partial P}{\partial z}= \\
& -f_{w z}-\alpha \rho_{v} g-M_{i z}+U_{i z}{ }^{\Gamma}
\end{aligned}
$$

The vapor mass conservation equation is given by:

$$
\frac{\partial}{\partial t}\left(\alpha \rho_{\mathrm{V}}\right)+\frac{\partial}{\partial z}\left(\alpha \rho_{\mathrm{v}} \mathrm{U}_{\mathrm{V} z}\right)+\frac{\partial}{\partial r}\left(\alpha \rho_{\mathrm{V}} \mathrm{U}_{\mathrm{vr}}\right)=\Gamma
$$

and this can be substituted into equation 2.39 to yield the non-conservative form

$$
\begin{aligned}
& \alpha \rho_{\mathrm{v}} \frac{\partial \mathrm{U}_{\mathrm{V}}}{\partial \mathrm{t}}+\alpha \rho_{\mathrm{v}} \mathrm{U}_{\mathrm{vz}} \frac{\partial \mathrm{U}_{\mathrm{v} z}}{\partial \mathrm{z}}+\alpha \rho_{\mathrm{v}} \mathrm{U}_{\mathrm{vr}} \frac{\partial \mathrm{U}_{\mathrm{V}}}{\partial \mathrm{r}}+\alpha \frac{\partial \mathrm{P}}{\partial \mathrm{z}}= \\
& -\mathrm{f}_{\mathrm{wz}}-\alpha \rho_{\mathrm{v}} \mathrm{g}-\mathrm{M}_{1 \mathrm{z}}+\mathrm{U}_{1 \mathrm{z}} \Gamma-\mathrm{U}_{\mathrm{vz}} \Gamma
\end{aligned}
$$

Next $M_{v z}^{\prime}$ is defined such that

$$
\mathrm{M}_{v z}^{\prime}=-\mathrm{M}_{1 z}+U_{1 z} \Gamma-U_{v z} \Gamma
$$


where

$$
\begin{aligned}
& M_{1 z}=K \cdot\left(\ddots_{\mathrm{V} z}-{ }_{\ell z}\right) \\
& K=\text { interfacial momentum exchange coeff } 1 \text { cient }
\end{aligned}
$$

$M_{\mathrm{V} z}^{\prime}$ can be rearranged by the following procedure:

$$
\begin{aligned}
& \mathrm{M}_{\mathrm{Vz}}=-\mathrm{K}\left(\mathrm{U}_{\mathrm{Vz}}-\mathrm{U}_{\ell \mathrm{z}}\right)+\left[\eta \mathrm{U}_{\ell \mathrm{z}}+(1-\eta) \mathrm{U}_{\mathrm{Vz}}-\mathrm{U}_{\mathrm{Vz}}\right] \cdot \Gamma \\
& =-K\left(U_{V Z}-U_{\ell z}\right)+\left[n\left(U_{\ell z}-U_{V Z}\right)+U_{V z}-U_{V z}\right] \cdot \Gamma \\
& =-(K+\eta \Gamma) \cdot\left(U_{v z}-U_{\ell z}\right)
\end{aligned}
$$

One can follow the same procedure for the liquid phase momentum equation to obtain the non-conservative form, which is:

$$
\begin{aligned}
& (1-\alpha) \rho_{\ell} \frac{\partial U}{\partial t}^{Z z}+(1-\alpha) \rho_{\ell} U_{\ell z} \frac{\partial U}{\partial z}^{U}+(1-\alpha) \rho_{\ell} U_{\ell r} \frac{\partial U}{\partial r}^{U}+ \\
& (1-\alpha) \frac{\partial P}{\partial r}=-f_{W z}-(1-\alpha) \rho_{\ell} g+M_{i z}-U_{i z} \Gamma+U_{\ell z} \Gamma
\end{aligned}
$$

Defining

$$
M_{\ell z}^{\prime}=M_{i z}-U_{i z} \Gamma+U_{\ell z} \Gamma
$$

$$
\text { where } \quad M_{i z}=K \cdot\left(U_{v z}-U_{\ell z}\right)
$$

one can simplify $M_{\ell z}^{\prime}$ to obtain

$$
\mathrm{M}_{\ell z}^{\prime}=(K-(I-n) \Gamma) \cdot\left(U_{v z}-U_{\ell z}\right)
$$

In order to better interpret these results, consider a 
situation where $\eta=0.5$ so that $U_{i}=\left(U_{\ell z}+U_{v z}\right) / 2$, and where $U_{v}>U_{l}$. For an evaporating condition $(\Gamma>0)$, the terms $M_{v z}^{\prime}$ and $M_{\ell z}^{\prime}$ both decrease. The vapor phase bulk momentum decreases by picking up slower particles $\left(U_{i}<U_{V}\right)$ and the liquid phase bulk momentum decreases by losing particles traveling at $U_{I}>U_{\ell}$.

In a condensing condition, both $M_{V z}^{\prime}$ and $M_{\ell z}$ increase. The vapor phase bulk momentum increases by losing its slow particles and the liquid phase gains momentum by receiving fast particles.

A comparison of $K$ and $n \Gamma$ verses void fraction was made in order to access the importance of this phenomenon. As can be seen in figure 2.6, for values of $\alpha>0.88$, the term is the dominating one. This is a desired result, since as the liquid becomes entrained in the vapor phase, the slip ratio should decrease as the liquid particles become borne in the vapor phase. Parameters used for this comparison are given in Table 2.1

To determine what effect this modification actually has on NATOF-2D simulations, a sodium boiling transient was run with the new correlation (with $\eta=0.5$ ), and compared to the same transient without it. The results showed an insignificant difference for the full range of void fractions.

Simulations were also run in which $n$ was varied in the range from 0.0 to 1.0 . The only noticeable difference was 


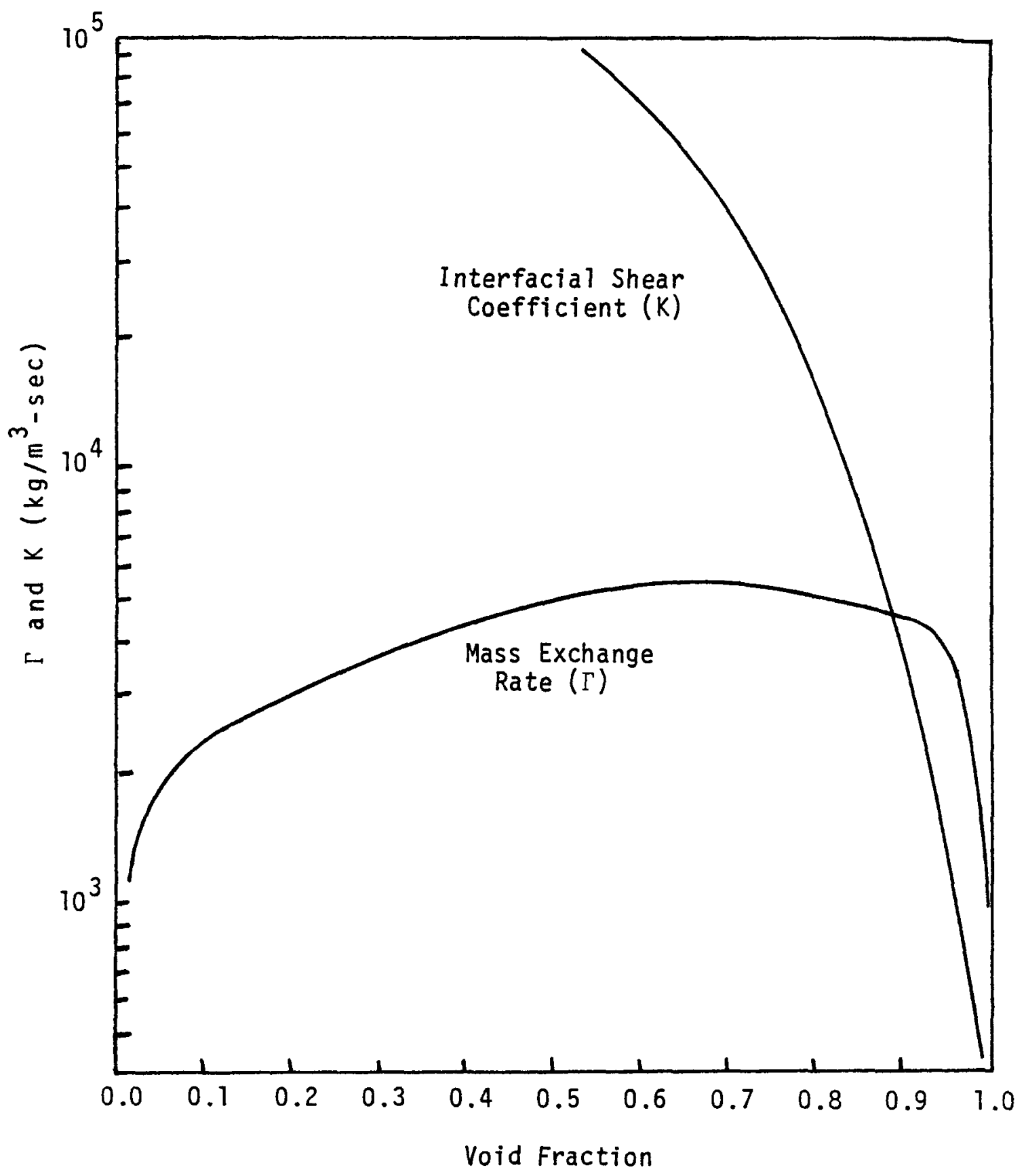

Figure 2.6 Values of $\Gamma$ and $K$ as a Function of Void Fraction for Typical LMFBR Transient Conditions 
$-52-$

Table 2.1

Parameters Used in $K$ versus $n \Gamma$ Comparison

Pressure $\left(N / m^{2}\right)$

Saturation Temperature $\left({ }^{\circ} \mathrm{K}\right)$

Fuel Pin Diameter ( $m$ )

Hydraulic Diameter (m)

Pitch/Diameter

Vapor Density $\left(\mathrm{kg} / \mathrm{m}^{2}\right)$

Vapor Velocity $(\mathrm{m} / \mathrm{s})$

Liquid Velocity $(\mathrm{m} / \mathrm{s})$

$T_{\ell}-T_{\text {sat }}\left({ }^{\circ} K\right)$
2. $\times 10^{5}$

1235.59

$5.842 \times 10^{-3}$

$4.223 \times 10^{-3}$

1.25

0.53

25.0

$5 \cdot 9$

2.0 
that for $n=0.0$ the vapor velocity was lower than for $n=1.0$, and for $n=0.0$ the liquid velocity was higher than for $n=1.0$. Since these results are for a region where condensation is occurring $(\Gamma<0)$, this was expected. Refering to equation 2.44 , the term $(K+\eta \Gamma)$ is smallest when $\eta=1$. Thus the vapor phase isn't slowed down by the liquid phase as much. Refering to equation 2.46 , the term $(K-(1-\eta) \Gamma)$ is smallest for $\eta=1$, and so the liquid phase is not dragged as much by the vapor phase. Hence, the lower velocity. 
$-54-$

2.6 Programming Information

Both the new mass exchange rate and energy exchange rate were incorporated into subroutine NONEQ.

The momentum exchange rate was incorporated into subroutine WS. Since $\Gamma$ is required in this formulation, and since it must be evaluated at the previous time step, the value of the mass exchange rate is stored in subroutine ONESTP for use in the following time step. 
Chapter 3

FLUID CONDUCTION MODEL

\section{I Introduction}

Some of the previous sodium boiling transients simulated with NATOF-2D have shown a large difference in the fluid termperature between the central channels and the edge channel. A small variation is expected since the edge channel experiences heat losses to the hexcan container, and since there is usually a lower power to flow ratio in the ouside channel. However, whereas in the W-1 SLSF experiments a radial temperature variation of $10^{\circ} \mathrm{C}$ was reported for steady state operation / 9 /, NATOF-2D predicted a difference of $60^{\circ} \mathrm{C} / 1 \%$

In LMFBR bundles, the fuel rods are helically wound with spacer wires. These wires act as a spacing agent between fuel rods, and tend to sweep the coolant transversely around the bundle. This results in turbulence and good mixing of the coolant. NATOF-2D, as originally developed, is unable to simulate this phenomenom. The radial velocities found in NATOF-2D are due solely to the radial pressure gradient, which in most cases is rather small in magnitude. Since mass transfer between cells was the only mechanism available for energy exchange, the large temperature gradients persisted. When boiling occurs, the 
previously mentioned sweeping effects become negligible compared with the expansion of the vapor phase.

Therefore to account for the observed temperature profile, radial heat conduction has been incorporated into the code. The heat transfer between cells has been modelled in terms of "effective" conduction between the fluid in adjacent cells. Besides modelling the pure conduction effects, the formulation will also be used to account for mixing and diffusive effects in the fuel bundle. Axial heat conduction has been neglected since the the high axial velocities allow the effects of convection to dominate any conductive effects. Also, the low conductivity of the vapor phase makes any vapor-liquid or vapor-vapor radial heat transfer effects negligible. This chapter will present the methodology for calculating radial heat conduction, and offer typical values for the effective nusselt number for conduction. 


\subsection{Formulation}

For the arrangment of cells shown in figure 3.1 , the total heat transfer rate to cell $i$ can be expressed as the sum of the heat transfer rates through each of its two faces. In this formulation, the heat flux is given by an effective heat transfer coefficient times the difference in the temperature of the adjacent cells. Written explicitly, this becomes:

$$
q_{i T}=q_{i-1, i}+q_{i+1, i}
$$

where

$$
\begin{aligned}
& q_{1-1,1}=A_{1-1,1} h_{i-1,1}\left(T_{1-1}-T_{i}\right) \\
& q_{1+1,1}=A_{1+1,1} h_{1+1,1}\left(T_{1+1}-T_{i}\right)
\end{aligned}
$$

and

$$
\begin{aligned}
q_{1 T} & =\text { total heat transfer rate to cell } i \\
q_{1-1, i} & =\text { heat from cell } i-1 \text { to cell } i \\
q_{1+1, i} & =\text { heat from cell } i+1 \text { to cell } i \\
h_{1-1,1}= & \text { effective heat tranxfer coefficient between } \\
& \text { cell } i-1 \text { and cell } i \\
T_{1} & =\text { temperature of cell } i \\
A_{1+1, i} & \text { intercell area }
\end{aligned}
$$

On either side of the interface seperating two adjacent cells, a heat transfer coefficient has been defined with the form: 


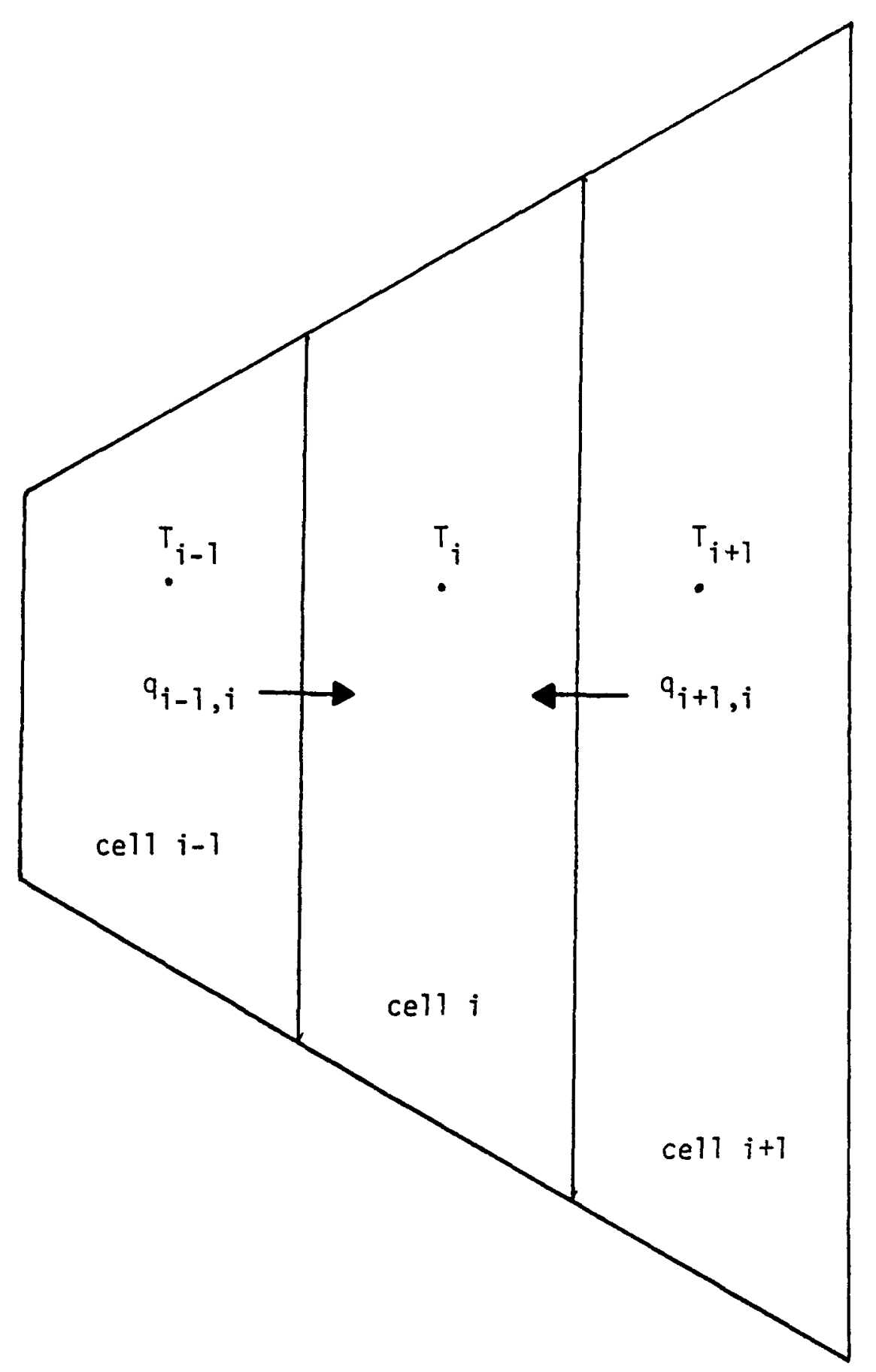

Figure 3.1 Top View of Fluid Channels Showing the Radial Heat Transfer Between Them 


$$
\mathrm{h}_{1}=\mathrm{Nu} \cdot \frac{\mathrm{K}_{\ell}}{1 / 2 \mathrm{D}_{\mathrm{c}}}
$$

where

$$
\begin{aligned}
\mathrm{Nu} & =\text { effective nusselt number } \\
K_{\ell} & =\text { conductivity of the liquid in cell } i \\
D_{c} & =\text { conductive diameter of cell } i \\
& =\frac{4 \cdot A_{f l o w}}{P_{c}} \\
P_{c} & =\text { perimeter of fluid-fluid conduction }
\end{aligned}
$$

Conservation of energy. requires that the heat flux from cell $i$ to the interface of cells $i$ and $i+1$ be equal and opposite to the heat flux from cell $i+1$ to the interface, and so an interface temperature, $T_{\text {Int }}$, can be defined such that

$$
h_{1}\left(T_{\text {Int }}-T_{1}\right)=-h_{1+1}\left(T_{\text {int }}-T_{1+1}\right)
$$

Solving for the interface temperature yields

$$
T_{\text {int }}=\frac{h_{1} T_{1}+h_{1+1} T_{1+1}}{h_{1}+h_{i+1}}
$$

Since the heat flux to the interface from cell $i$ is the same as the heat flux between cells $i$ and $i+1$, the right hand side of equation 3.5 can be equated to equation 3.3 to yield:

$$
h_{1}\left(T_{\text {int }}-T_{1}\right)=h_{i+1, i}\left(T_{i+1, i}-T_{i}\right)
$$


Substituting in equation 3.6 for $T_{i n t}, h_{i+1,1}$ can now be solved for. The result is:

$$
h_{i+1, i}=\frac{h_{i+1} \cdot h_{1}}{h_{i+1}+h_{i}}
$$

Considering the case where $h_{i}=h_{i+1}$, equation 3.8 reduces to

$$
\begin{aligned}
h_{1+1,1} & =1 / 2 \cdot h_{1+1} \\
& =N u \cdot \frac{K_{\ell}}{D_{c}}
\end{aligned}
$$

as one would expect.

In summary, the methodology of this approach is to calculate $h$ as given by equation 3.4 for each cell, and then use these values to solve for $h_{i+1,1}$. Once this is accomplished, equation 3.1 can be evaluated for each cell. 


\subsection{Intercell Areas}

NATOF-2D is structured in such a way that the boundary between cells lies at the plane connecting the fuel pin centerlines as one travels radially outward. An illustration of this, along with the numbering of the boundaries is given in figure 3.2 .

Treating the bundle as a porous body, the radial heat transfer area becomes dependent only on the radial distance $r_{n}$. Thus

$$
A r=A r^{*} \cdot r_{n}
$$

where

$$
\begin{aligned}
A r= & \text { volumetrically averaged radial area between } \\
& \text { cell boundaries } \\
A r^{*}= & \text { radial area constant } \\
r_{n}= & \sqrt{3} / 2 \cdot n \cdot p \\
n= & \text { row number }(1,2,3, \ldots \ldots) \\
p \quad= & \text { fuel rod pitch }(m)
\end{aligned}
$$

Considering for the moment the unit cell shown in figure $3.3, \mathrm{Ar}^{*}$ can be solved for by requiring that

$$
\begin{aligned}
V_{c e l l} & =\int_{0}^{r_{1}} A r \cdot d r \\
& =\int_{0}^{r_{1}} A r^{*} \cdot r_{n} d r \\
& =A r^{*} \cdot r_{1}^{2} / 2
\end{aligned}
$$




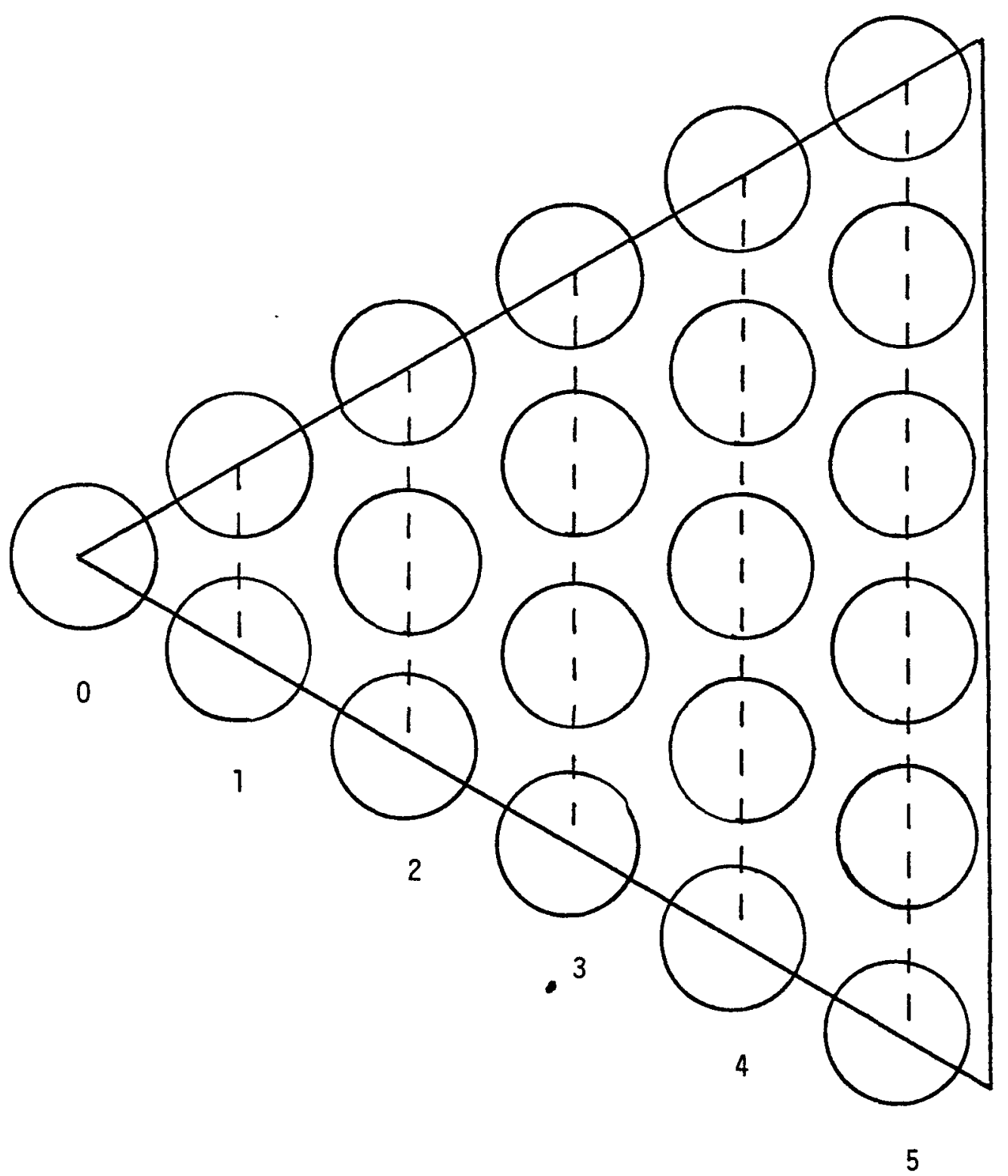

Figure 3.2 Top View of Fluid Channels Showing Radial Cell Boundary Numbering Scheme 


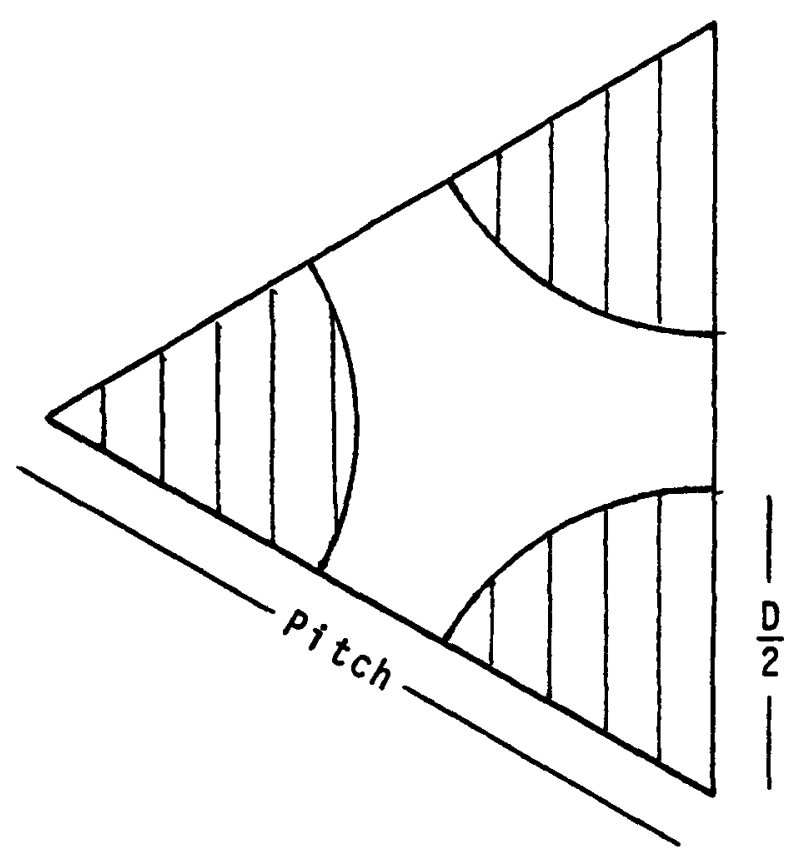

Figure 3.3 Unit Ce11 Used in NATOF-2D 
The volume of the unit cell is given by

$$
V_{\text {cell }}=\frac{1}{2}\left(P^{2} \cdot \frac{\sqrt{3}}{2}-A_{p i n}\right) \Delta z
$$

where

$$
\begin{aligned}
A_{p i n} & =\text { area of the pin and wirewrap } \\
& =\frac{\pi}{4}\left(D^{2}+d^{2}\right) \\
D & =\text { fuel pin diameter } \\
d & =\text { wire wrap diameter } \\
\Delta z & =\text { axial height of cell }
\end{aligned}
$$

Using the relation in equation 3.10 yields:

$$
\mathrm{Ar}^{*}=\frac{4}{3}\left(\frac{\sqrt{3}}{2}-\frac{\mathrm{A}_{\mathrm{pin}}}{\mathrm{P}^{2}}\right) \Delta z
$$

The final form of the radial heat transfer area is then

$$
\operatorname{Ar}=\left(1-\frac{2}{\sqrt{3}} \cdot \frac{A_{p \text { in }}}{P^{2}}\right) n P \Delta z \quad(n=1,2, \ldots)
$$

When this formulation is implimented in NATOF-2D, it is necessary to divide the total heat transferred by the volume of the cell so that the term will appear as a heat source term in the energy conservation equation. For a cell whose boundaries lie at $n_{j-I}$ and $n_{j}$, excluding the edge cell, the total volume is given by

$$
V=\left(P^{2} \cdot \frac{\sqrt{3}}{2}-A_{p i n}\right) \cdot \frac{n_{j}^{2}-n_{j-1}^{2}}{2}
$$




\subsection{Implementation Form}

Up until this point, no mention has been made of the time step discretinization used in the code for this formulation. In this section, the options available in NATOF-2D and the advantages and limitations of each are covered.

The first option is to treat the calculation in a fully explicit manner such that

$$
q_{1 T}^{n+1}=A_{1-1, i} h_{1-1, i}^{n} \frac{n}{\left(T_{1-1}-T_{1}\right)}+A_{i+1, i} h_{i+1, i}^{n}\left(T_{i+1, i}^{n} T_{i}^{n}\right)
$$

where the superscript $(n+1)$ refers to the present time step, and $(n)$ refers to the previous time step. Since all terms on the RHS of equation 3.15 are known values, this option requires that the calculation be performed only once per time step. Thus the cpu costs for the explicit calculation are low. It also ensures strict energy conservation since

$$
q_{1-1,1}=-q_{1,1-1^{*}}
$$

A fourier stability analysis performed on equation 3.15 shows that this formulation limits the time step size to

$$
\Delta t \leq \frac{\Delta r^{2}}{2 \cdot \alpha \cdot N u}
$$

where

$$
\alpha=\frac{k}{\rho c_{p}}
$$

In most cases, the convective time limit $\left(\Delta t \leq \Delta z / \mathrm{U}_{z}\right)$ is more restrictive than the conductive limit. However, a 
feature has been implimented into the code which calculates the time step limitation when the explicit calculation is utilized, and maintains a time step value below the conductive limit.

The second option available is to treat the radial heat transfer calculation semi-implicitly. The form of the calculation is:

$$
q_{1 T}^{n+1}=A_{1-1,1} h_{1-1,1}^{n}\left(T_{i-1}^{n}-T_{i}^{n+1}\right)+A_{i+1, i} n_{i+1, i}^{n}\left(T_{i+1}^{n}-T^{n+1}\right)
$$

A stability analysis applied to equation 3.17 shows that the scheme is unconditionally stable, and therefore poses no constraint to the time step size. However, it does have two limitations. The first is that it fails to conserve energy since the relation

$$
n_{i-1, i}^{n}\left(T_{1-1}^{n}-T_{i}^{n+1}\right)=-n_{i, i-1}^{n}\left(T_{i}^{n}-T_{i-1}^{n+1}\right)
$$

will not be satisfied in general. The second limitation is that this calculation needs to be performed once per newton iteration, instead of once per time step. Thus, the cpu usage will be greater than the explicit method.

A fully implicit calculation of the form

$$
q_{1 T}^{n+1}=A_{i-1, i} h_{1-1,1}^{n}\left(T_{i-1}^{n+1}-T_{i}^{n+1}\right)+A_{1+1,1} n_{i+1,1}^{n}\left(T_{i+1}^{n+1}-T_{1}^{n+1}\right)
$$

cannot be utilized by NATOF-2D since the solution scheme of 
the code requires that the only linkage between cells be by the pressures of the cells. A formulation such of this would also link the cell temperatures.

As can be seen in figure 3.2, all interior cells are similar, and therefore we are somewhat justified in using the same effective nusselt number $\mathrm{Nu}_{1}$. The edge channel, however, has a quite different shape, and so a second nusselt number, $\mathrm{Nu}_{2}$, is used to take into account the effects of any differences. 


\subsection{Experimental Calibration}

In order to obtain a practical value for the effective nusselt number for radial heat conduction, a steady-state, single phase sodium experiment was chosen from the Westinghouse Blanket Heat Transfer Test Program / $2 /$. The heat transfer test section was a mockup of an LMFBR blanket assembly. The test section consisted of 61 rods contalned in a hexagonal duct. Each rod delivered an axial heat output approximating a chopped cosine distribution with a 1.4 maximum-to-average ratio over a $114.3 \mathrm{~cm}$. length. In test No. 544, the total bundle power was $440 \mathrm{kw}$, and the radial power distribution was uniform. Test parameters are given in Table 3.1, and the input for the NATOF-2D simulation is given in appendix $C$.

The test procedure was to adjust the test loop operating parameters until the desired sodium flow and inlet temperature was achieved. At this point, power to the bundle was gradually increased until the test section power gradient and temperature rise attained operating conditions. The test section was then allowed to achieve a steady state configuration, at which point data was collected. For test No. 544, the temperature profile across the bundle at three different axial levels was recorded. These levels corresponded to the heated zone midplane, the outlet of the heated zone, and 25 inches downstream of the heated zone.

In the NATOF-2D simulation, the proper flow and total 
Table 3.1

\begin{abstract}
Westinghouse Blanket Heat Transfer Test Program Rod Bundle Test Section Design / 2 /
\end{abstract}

\title{
PARAMETER
}

Number of Rods

Rod Diameter (cm)

1.32

Length of Heated Zone $(\mathrm{cm})$

114.3

Total Bundle Length $(\mathrm{cm})$

265 .

Wire Wrap Spacer Diameter $(\mathrm{cm})$

Triangular Rod Pitch

1.43

Wire Wrap Pitch $(\mathrm{cm})$

10.16

Pitch to Diameter Ratio

1.082

Duct Inside Diameter $(\mathrm{cm})$

Axial Power Distribution, Cosine

Max/Avg

Sodium Inlet Temperature $\left({ }^{\circ} \mathrm{C}\right)$

Sodium Flow Rate $\left(\mathrm{m}^{2} / \mathrm{hr}\right)$

Run 544

Run 545

12.0

Test Bundle Power ( $\mathrm{kw}$ )

440 
enthalpy rise through the core was established. Then the nusselt numbers were varied until the temperature profile obtained matched as closely as possible the experimental results. As the effective nusselt number was increased, the radial temperature profile at the end of the heated section became flatter, as shown in figure 3.4. A comparison between the experimental results and the NATOF-2D simulation for different elevations is given in figures $3.5,3.6$ and 3.7 .

From this experiment, the recommended values for the effective nusselt number are

$$
\begin{aligned}
& N u_{1}=22 \\
& N u_{2}=28
\end{aligned}
$$

A second experiment from the same series of tests was simulated by NATOF-2D in order to verify the generality of the previous results. This was Run No. 545. In this test, the same total power was used as before, but the radial power distribution was varied to give a power skew which peaked at the edge pins and was at a minimum at the center. The normalized heat input per rod is shown in figure 3.8 . The results for three different elevations are shown in figures $3.9,3.10$, and 3.11 . 


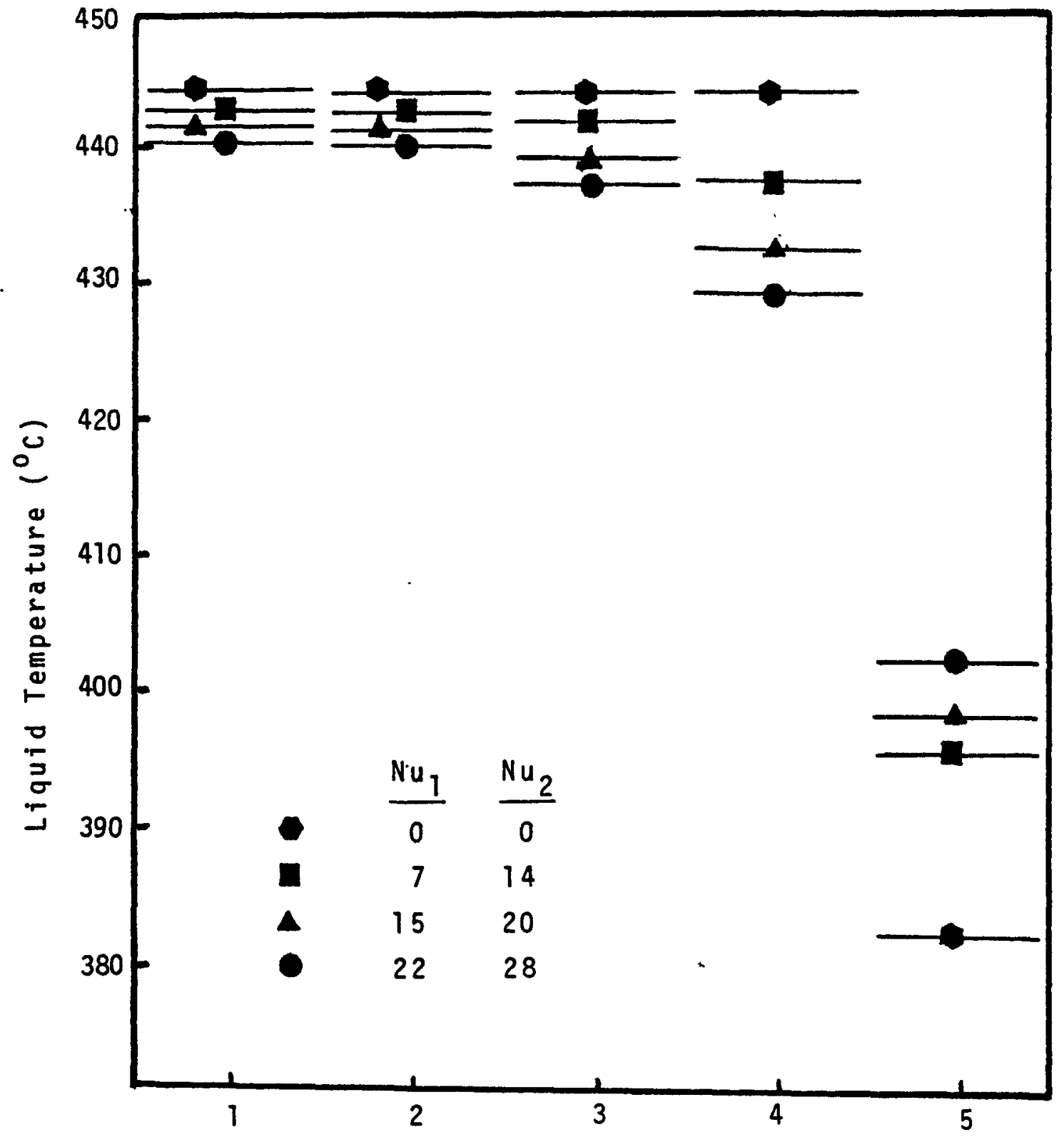

Channel Number

Figure 3.4 Radial Temperature Profiles at the End of the Heated Section for Various Effective Nusselt Numbers 


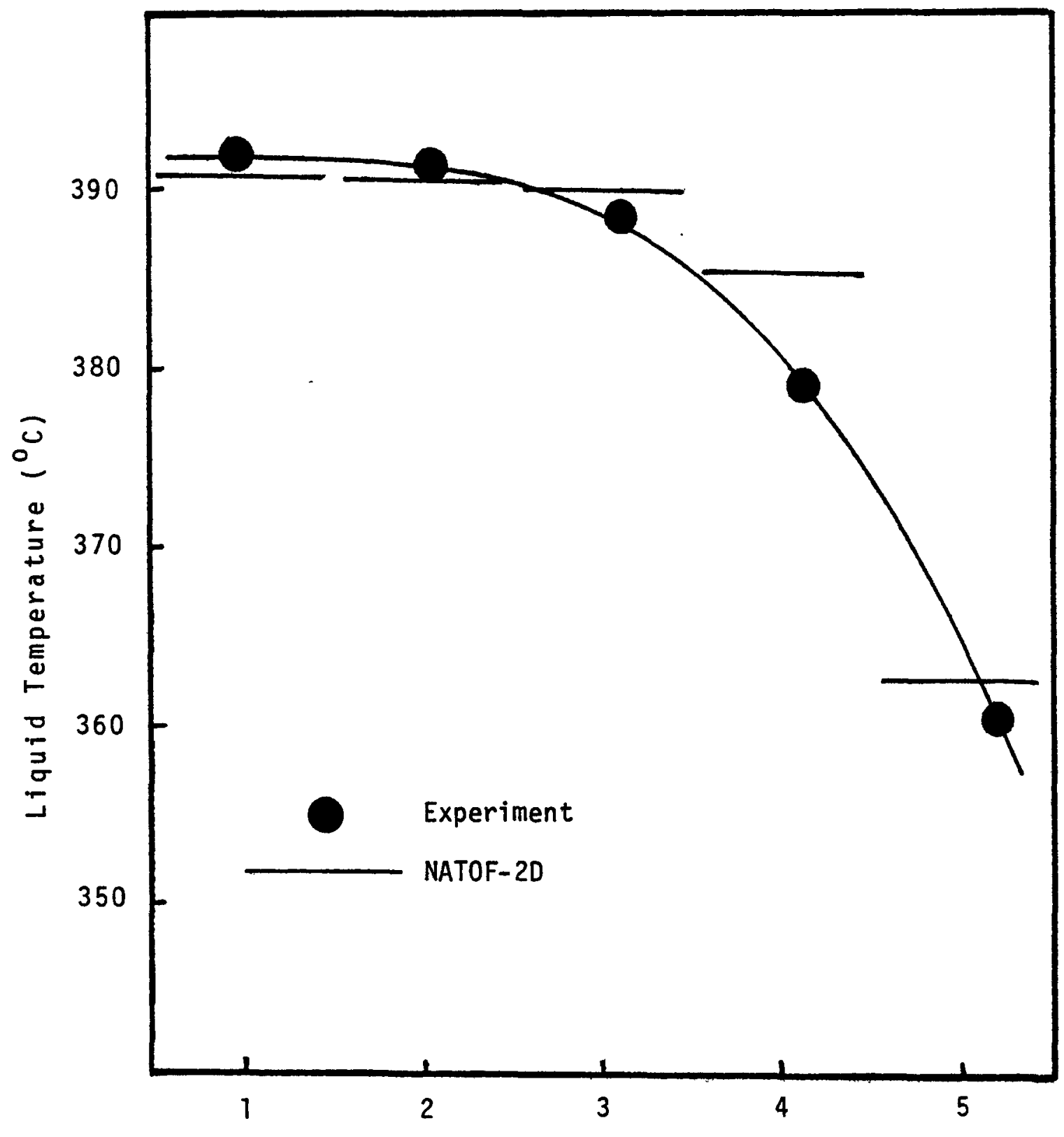

Channel Number

Figure 3.5 A Comparison Between Westinghouse Run 544 and NATOF-2D Radial Temperature Profiles at the Heated Zone Midplane for $\mathrm{Nu}_{1}=22$ and $\mathrm{Nu} u_{2}=28$ 


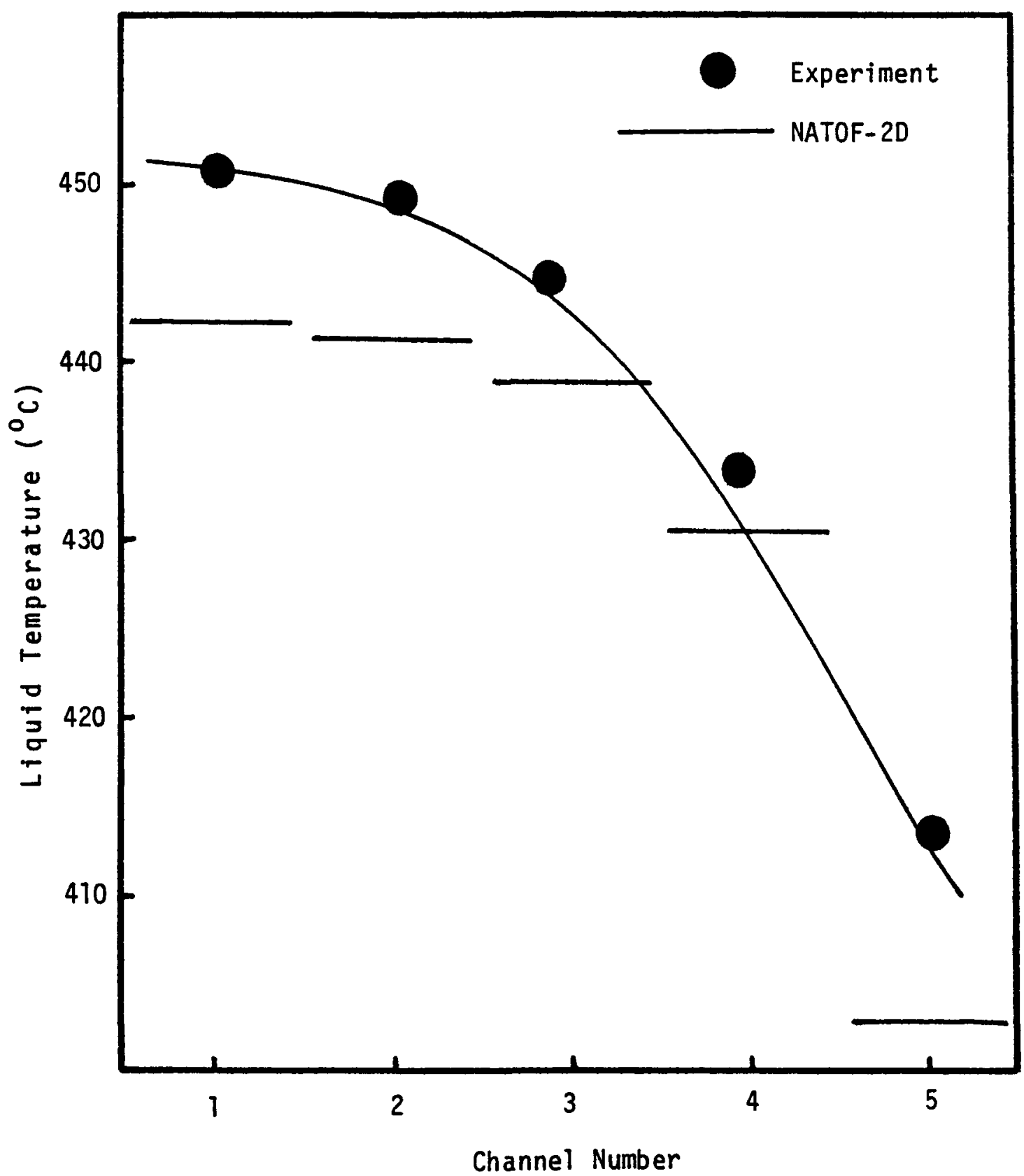

Figure 3.6 A comparison Between Westinghouse Run 544 and NATOF-2D Radial Temperature Profiles at the End of the Heated Zone for $N u_{1}=22$ and $N u_{2}=28$ 


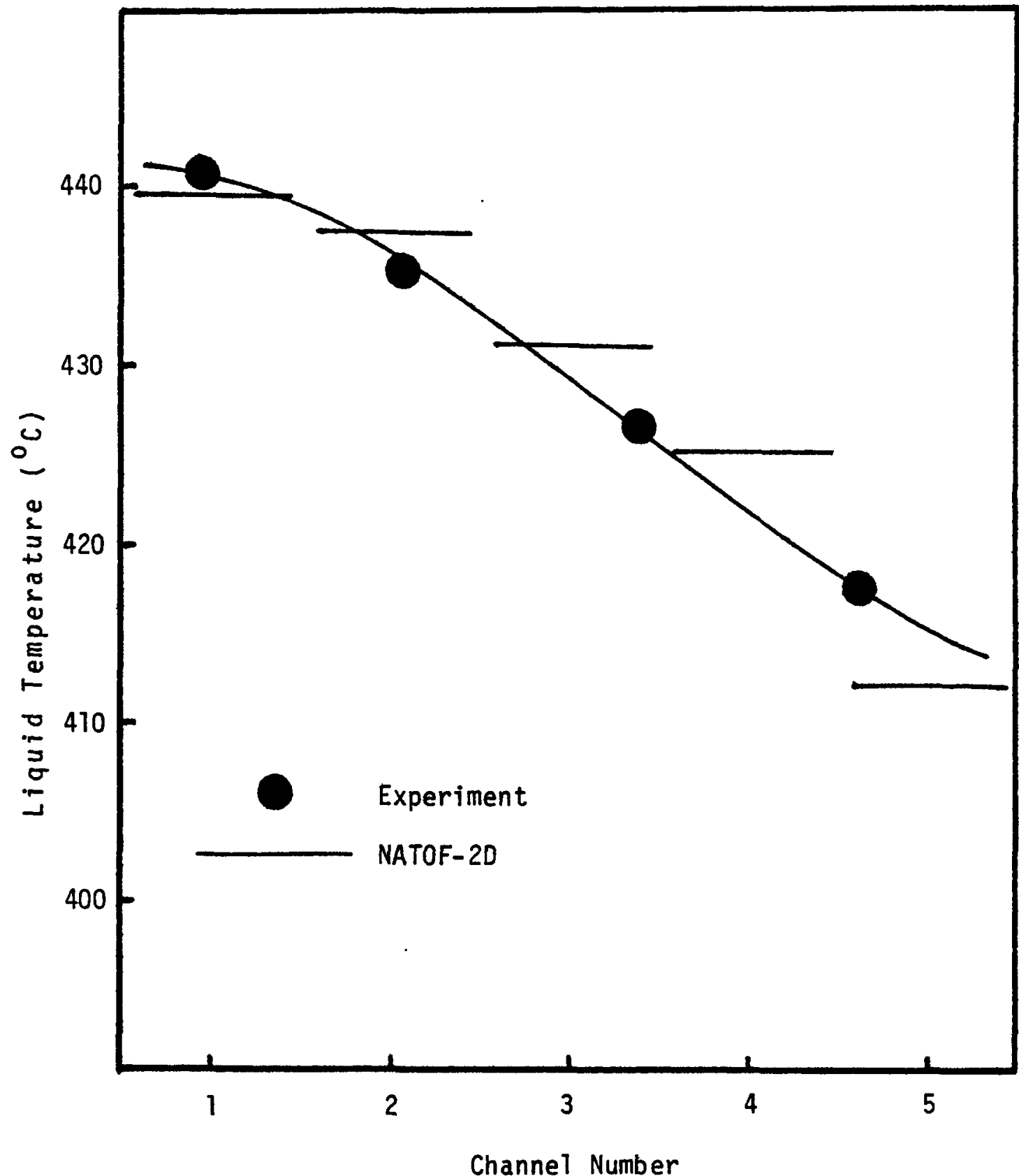

Figure 3.7 A Comparison Between Westinghouse Run 544 and NATOF-2D Radial Temperature Profiles 25 Inches Downstream of Heated Zone for $\mathrm{Nu}_{1}=22$ and $\mathrm{Nu}_{2}=28,2$, 


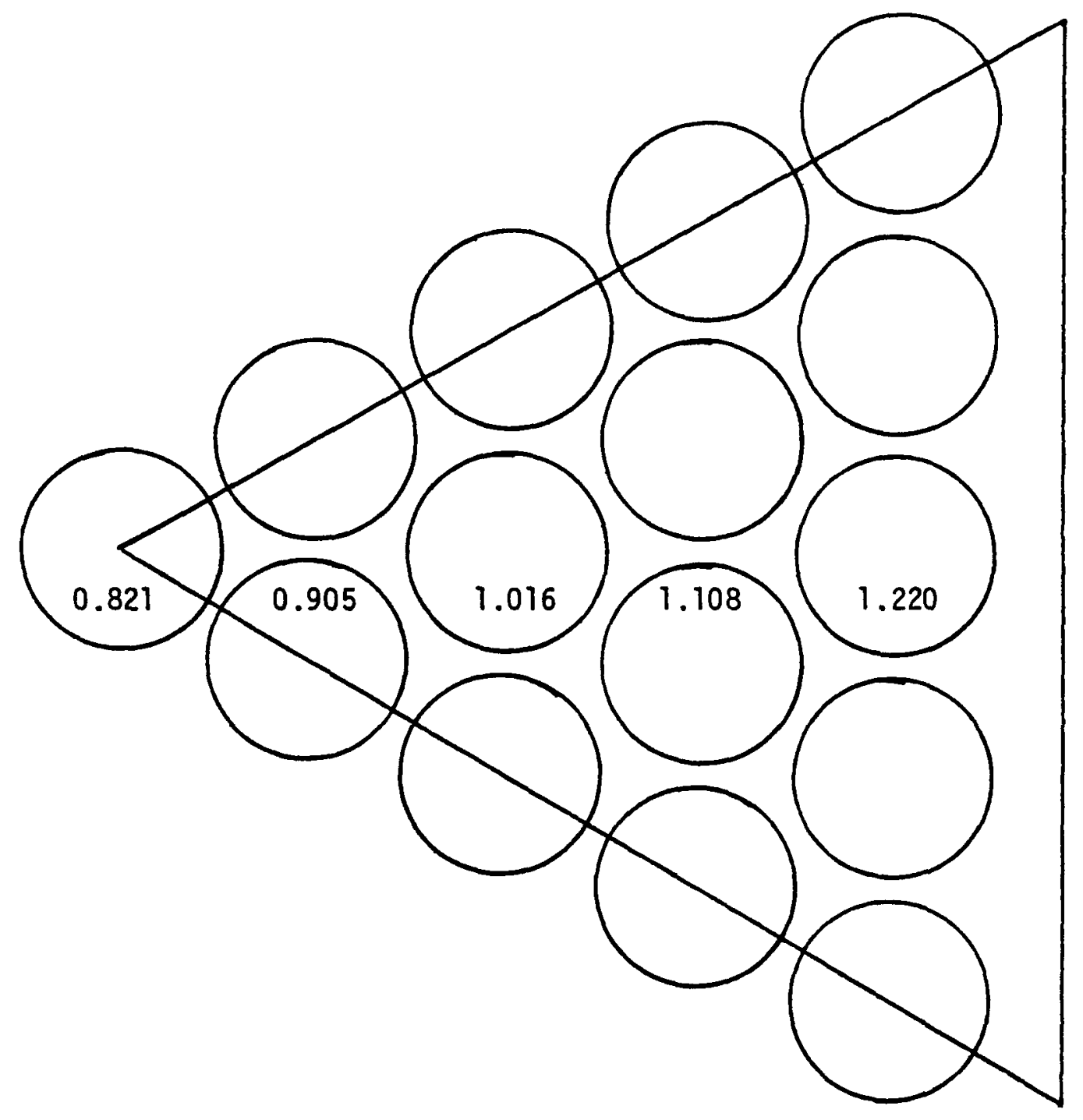

Figure 3.8 Normalized Heat Input per Rod for Westinghouse $B$ lanket Heat Transfer Test Program Run 545,2 / 


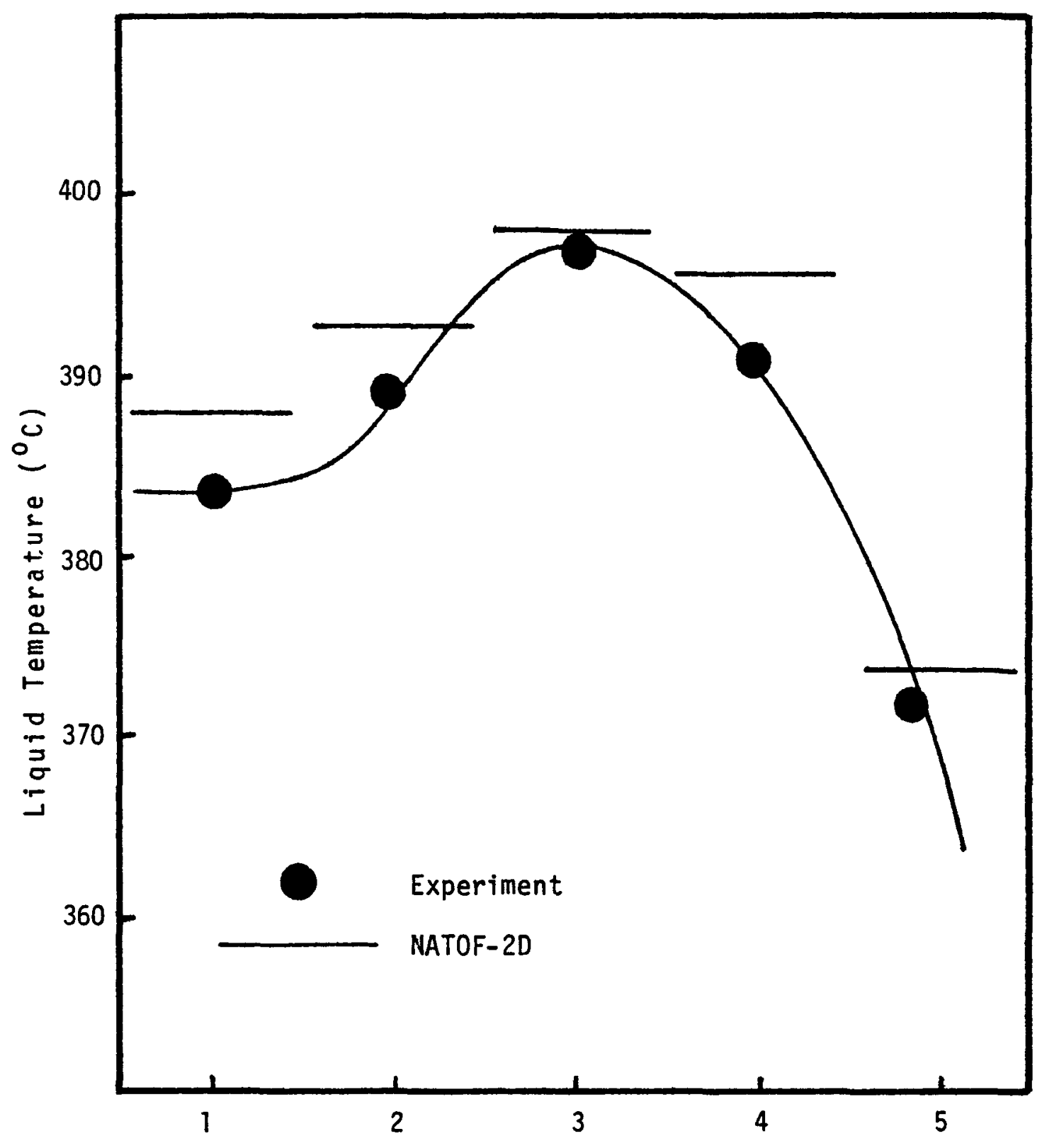

Channel Number

Figure 3.9 A Comparison Between Westinghouse Run 545 nad NATOF-2D Radial Temperature Profiles at the Heated Zone Midplane for $\mathrm{Nu}_{1}=22$ and $\mathrm{Nu} u_{2}=28$ 


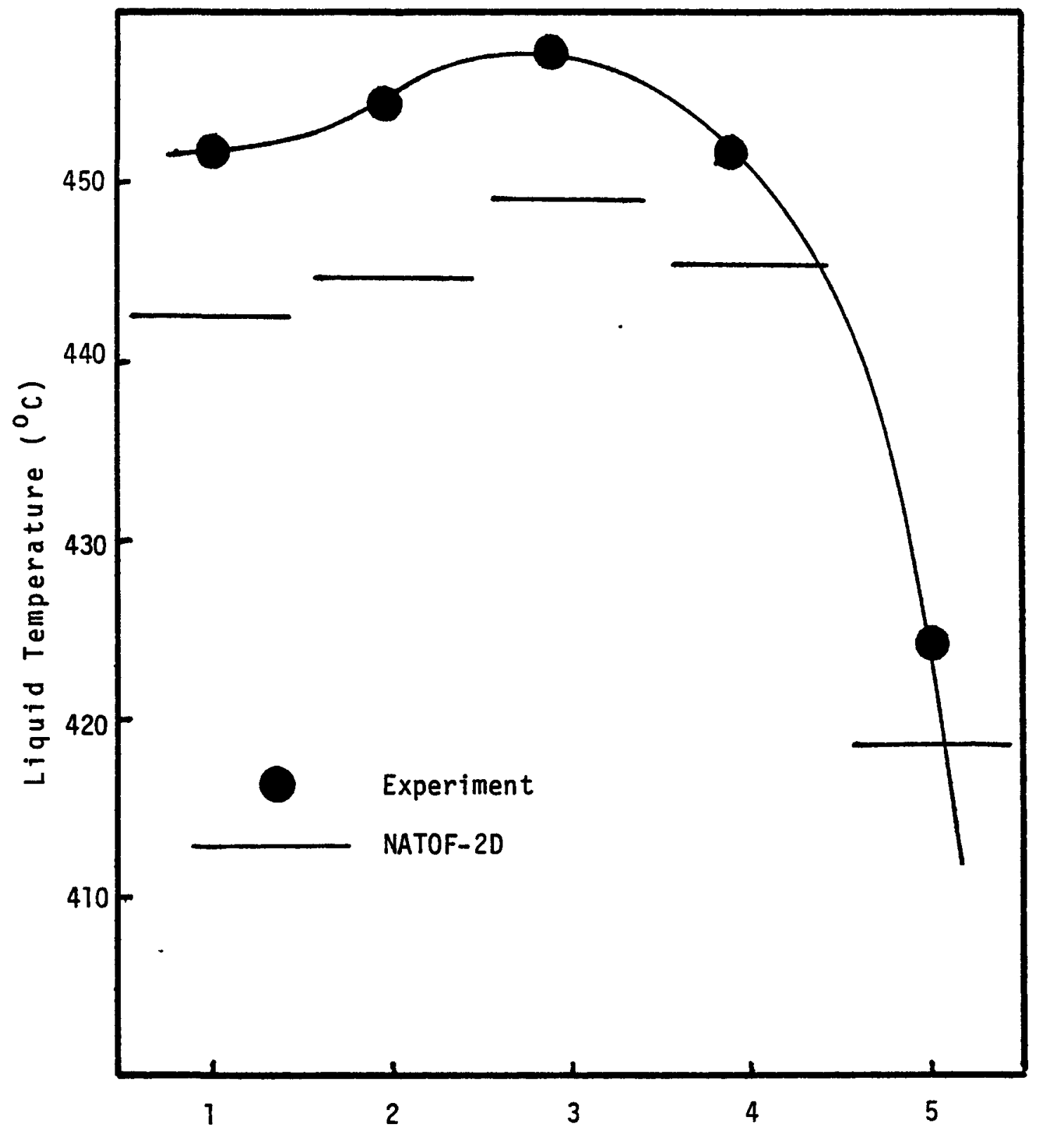

Channel Number

Figure 3.10 A Comparison Between Westinghouse Run 545 and NATOF-2D Radial Temperature Profiles at the End of the Heated Zone for $\mathrm{Nu}_{1}=22$ and $N u_{2}=28 / 2$ 


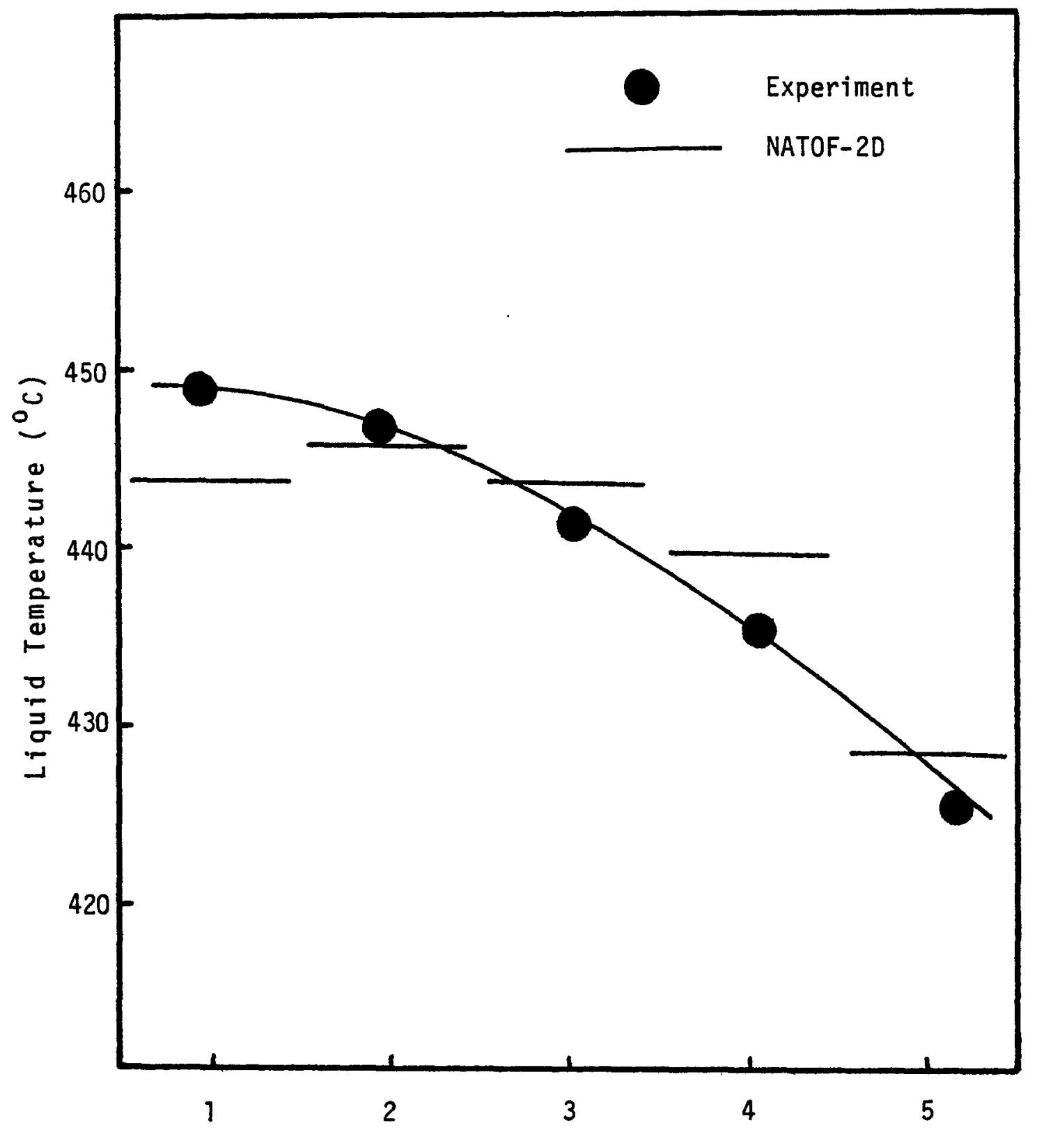

Channel Number

Figure 3.11 A Comparison Between Westinghouse Run 545 and NATOF-2D Radial Temperature Profiles 25 Inches Downstream of the Heated Zone for $\mathrm{Nu}_{1}=22$ and $\mathrm{Nu}_{2}=28 / 2 /$ 
3.6 A Comparison with Effective Conduction Mixing Lengths In this section, a comparison is made between the fluid-to-fluid conduction model implemented in NATOF-2D, and an analytic model developed to determine the effective mixing lengths for energy transport by conduction in subcahnnel codes. The model used for the evaluation of mixing lengths is from $M$. R. Yeung / $3 /$.

Before making the comparison, a brief outline is given of the method of $M$. R. Yeung to calculate the effective conduction mixing lengths. In this model, the heat transfer rate due to conduction between subcahnnels 1 and $j$ is given by the relation:

$$
Q_{1 j}=K_{\ell} \frac{S_{1 j}\left(\bar{T}_{1}-\bar{T}_{j}\right)}{l_{1 j}}=K_{\ell} \frac{S_{1 j}}{\ell_{1 j}^{*}}\left(\bar{T}_{1}-\bar{T}_{j}\right) \frac{I}{L_{1 j}}
$$

where

$$
\begin{aligned}
\mathrm{S}_{1 j}= & \text { the length of the common boundary } \\
\ell_{1 j}= & \text { the effective conduction mixing length } \\
l_{1 j}^{*}= & \text { the centroid-to-centroid distance of } \\
& \text { adjacent coolant channels } \\
\mathrm{K}_{\ell}= & \text { conductivity of the liquid phase } \\
L_{i j}= & \text { ratio of the effective conductive mixing } \\
& \text { length to the centroid-to-centroid distance } \\
= & \ell_{i j} / l_{i j}^{*}
\end{aligned}
$$

The effective conduction mixing length takes into account 
the fact that the actual heat flux due to conduction,

$$
\left.q^{\prime \prime}=-K_{\ell} \cdot \frac{\partial T}{\partial x}\right)_{\text {interface }}
$$

may be quite different than that given by equation 3.20 since subchannel codes deal with bulk temperatures while attempting to model a localized effect. As an illustration of this, possible temperature distributions which yield the same bulk temperature are shown in figure 3.12. As can be seen, $\partial T / \partial x)_{\text {int }}$ can vary widely.

Equation 3.20 can be rearranged to give:

$$
L_{i j}=K_{\ell} \cdot \frac{S_{1 j}}{l_{1 j}^{*}} \cdot \frac{\left(\bar{T}_{1}-\bar{T}_{j}\right)}{Q_{1 j}}
$$

Since the total heat transfer can be expressed as

$$
Q_{1 j}=\int_{s_{1 j}} q_{s}^{\prime \prime} d s
$$

equation 3.23 can be substituted into equation 3.20 , and also the dimensionless group $q$ ''a $/ 2 k$ and the rod radius $b$ can multiply and divide 3.20 to yield a form which can be analytically determined by evaluating each quantity. This form is:

$$
L_{i j}=\frac{s_{i j}}{l_{i j}^{*}} \cdot \frac{\frac{\left(\bar{T}_{1}-\bar{T}_{j}\right)}{q^{\prime \prime} \cdot a^{2} / 2 K_{\ell}}}{\int_{s} \frac{q_{s}^{\prime \prime}}{q^{\prime \prime} \cdot a^{2} / 2 b} d\left(\frac{s}{b}\right)}
$$

where

$$
a=\text { fuel pellet radius }
$$




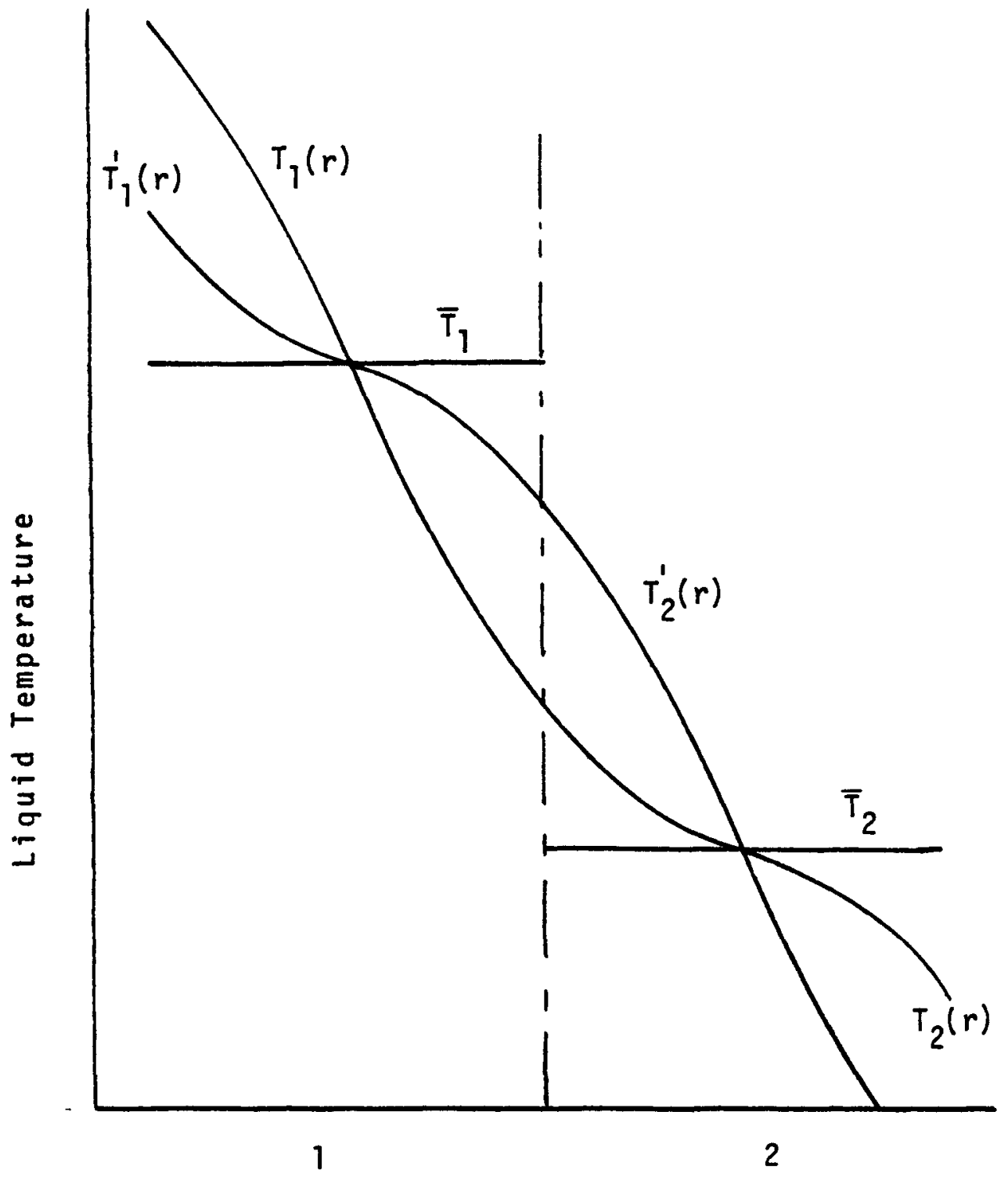

Channel Number

Figure 3.12 Possible Temperature Distributions which Yield the Same Cell Averaged Temperature 


$$
\begin{aligned}
b & =\text { fuel rod radius } \\
q_{S}^{\prime \prime} & =\text { heat flux at common boundary } \\
q^{\prime \prime \prime} & =\text { power density of the fuel }
\end{aligned}
$$

The method of evaluating each of these terms from the local temperature and power distributions and the geometry is given in reference / $3 /$. For the purposes of this comparison, it will suffice to give the results for a 19-pin hexagonal bundle. The geometry used for calculating the effective mixing lengths is shown in figure 3.13, where the dashed lines denote cell boundaries. The results of the calculation are give in Table 3.2 .

To compare the NATOF-2D formulation with the conductive mixing length results, the heat transfer of both formulations are equated such that

$$
A r \cdot h_{i j}\left(T_{i}-T_{j}\right)=K_{l} \cdot \frac{2 \cdot S_{i j} \Delta z}{l_{i j}^{*}}\left(\bar{T}_{i}-\bar{T}_{j}\right) \frac{I}{L_{i j}}
$$

where the factor 2 has been added to the RHS of equation 3.25 to take into account the fact that NATOF-2D divides the core into 6 symmetrical volumes, while Yeung's work divides the core into 12. Thus the area used in NATOF-2D is twice as large.

Assuming that $k_{\ell}$ is the same for both formulations, $\mathrm{h}_{i j}$ is then given by

$$
\mathrm{h}_{1 \mathrm{j}}=\frac{2 \cdot \mathrm{Nu} \cdot \mathrm{K}_{\ell}}{\mathrm{D}_{\mathrm{cI}}+\mathrm{D}_{\mathrm{c} 2}}
$$




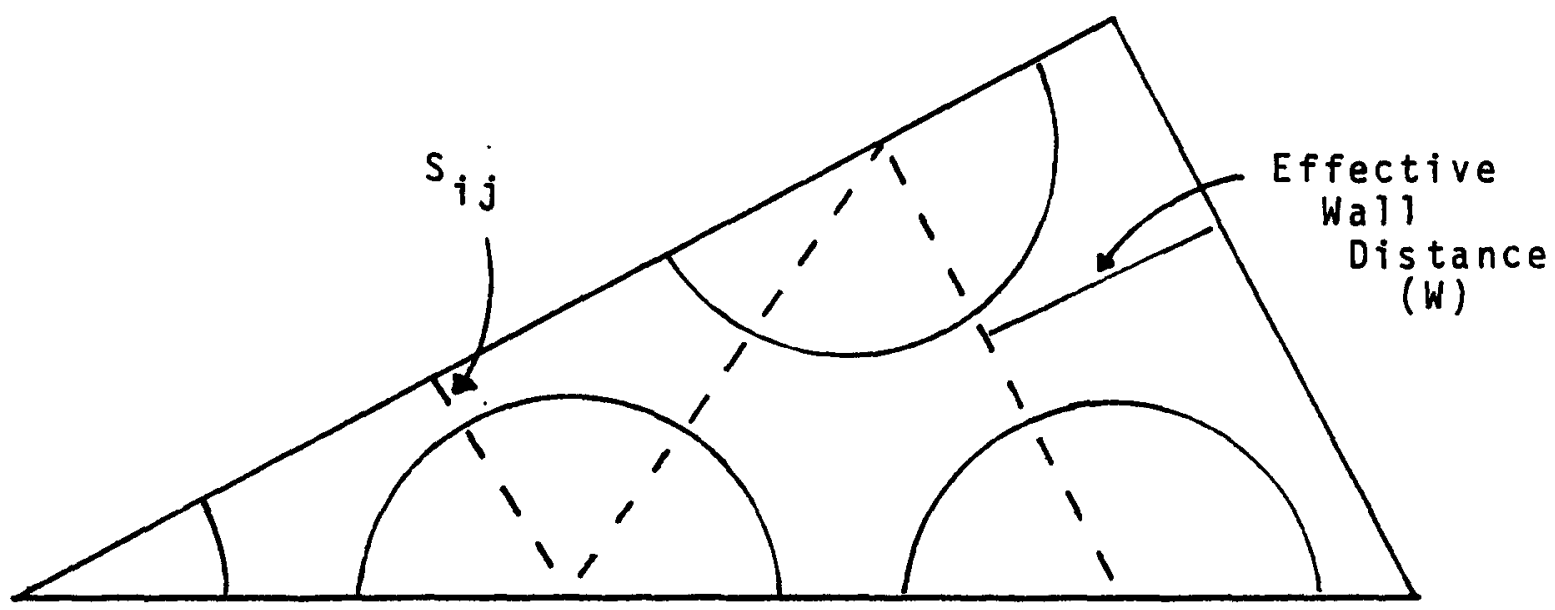

Figure 3.13 19-pin Cell Geometry Used for Calculation of Effective Mixing Lengths 
Table 3.2

$$
\begin{aligned}
& S_{12} / l_{12}^{*}=\frac{1}{2}\left(\frac{P}{D}-1\right) \frac{1}{\sqrt{3}} \frac{P}{D} \\
& S_{23} / l_{23}^{*}=\left(\frac{P}{D}-1\right) \frac{1}{\sqrt{3}} \frac{P}{D} \\
& S_{34} / l_{34}^{*}=\frac{\left.\frac{(P / D}{1}-1\right)}{2 \sqrt{3} \frac{P}{D}+\frac{\frac{1}{8}\left(\frac{2 W}{D}\right)^{2} \cdot \frac{P}{D}+\frac{1}{12 \sqrt{3}}\left(\frac{2 W}{D}\right)^{3}-\frac{1}{16}}{\frac{1}{2} \cdot \frac{P}{D} \cdot \frac{2 W}{D}+\frac{1}{4 \sqrt{3}} \cdot \frac{2 W}{D}-\frac{\pi}{12}}}
\end{aligned}
$$

Effective Mixing Lengths

$2 W / D$

Fuel Bundle

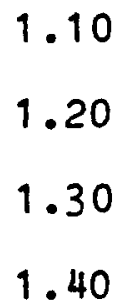

.85

.86

1.12

.75

.27

.57

.79

.77

.79

1.40

.791

.79

.81

Blanket Bundle

$\begin{array}{llll}1.04 & .69 & .69 & .645 \\ 1.08 & .69 & .69 & .68 \\ 1.10 & .69 & .69 & .69 \\ 1.20 & .69 & .69 & .70\end{array}$


Rearranging equation 3.25 in dimensionless form, and substituting in 3.26 yields:

$$
N u=\left(\frac{D_{c I}+D_{c 2}}{l_{i j}^{*}}\right)\left(\frac{S_{i j}}{A r}\right) \frac{1}{L_{i j}}
$$

As can be seen, both formulations are of the same form, and differ only by a constant multiple.

First, considering cells 1-2, each term of equation of 3.27 can be evaluated to get:

$$
\begin{aligned}
& \mathrm{S}_{12} / l_{12}^{*}=\frac{1}{2}\left(\frac{\mathrm{P}}{\mathrm{D}}-1\right) / \frac{1}{\sqrt{3}} \frac{\mathrm{P}}{\mathrm{D}} \\
& \mathrm{Ar}=\left(1-\frac{2}{\sqrt{3}} \frac{\pi}{4}\left(\frac{\mathrm{D}}{\mathrm{P}}\right)^{2}\right) \cdot \mathrm{P} \cdot \Delta \mathrm{z} \\
& \mathrm{D}_{\mathrm{Cl}}+\mathrm{D}_{\mathrm{Cl}}=4\left(1-\frac{2}{\sqrt{3}} \frac{\pi}{4}\left(\frac{\mathrm{D}}{\mathrm{P}}\right)^{2}\right) \frac{\sqrt{3}}{2} \mathrm{P}^{2} /(\mathrm{P}-\mathrm{D})
\end{aligned}
$$

Substituting these values into equation 3.27 yields

$$
\mathrm{Nu}=\frac{3}{\mathrm{~L}_{12}}
$$

From Table $3.2, \mathrm{~L}_{12}$ has a value equal to 0.69 . For a blanket bundle of the type simulated, this relation shows that the Nusselt number, due to conduction only, should be:

$$
\mathrm{Nu}=4.348
$$

For the edge cell, the complex geometry requires that each term be numerically evaluated. For this comparison, typical dimensions of a blanket assembly were used:

$$
D=1.320 \times 10^{-2} \text { meters }
$$




$$
\begin{aligned}
& P=1.426 \times 10^{-2} \text { meters } \\
& P / D=1.08 \\
& W \quad=P-D / 2=7.66 \times 10^{-3} \text { meters }
\end{aligned}
$$

The result is that

$$
\begin{aligned}
\mathrm{Nu} & =4.6322 \frac{\mathrm{I}}{\mathrm{L}_{34}} \\
& =6.617
\end{aligned}
$$

The results show that the effective nusselt number calculated from mixing length theory is much smaller than that required for the experiment calibration. This is to be expected for two reasons. The first is that the mixing length theory only takes into account fluid-to-fluid conduction effects, while the effective nusselt number is also accounting for turbulence and mixing. The second reason is that the mixing length results are specifically for 19 pin bundles, while the simulations were conducted for 61 pin bundles. It is expected that for smaller bundle sizes the effective nusselt numbers will also decrease. 
3.7 Programming Information

Two additional subroutines have been added to NATOF-2D. The first is subroutine QCOND which calculates the heat transferred per unit volume, and its derivative (when the implicit formulation is required). The second is subroutine HTRAN, which calculates the effective heat transfer coefficient.

The user specifies the type of calculation to be performed by specifying the sign of the nusselt numbers, which are a user input. A negative nusselt number refers to a semi-implicit calculation, while a positive nusselt number refers to a fully explicit calculation.

The nusselt numbers given in this chapter should be used as a gauge for the ones actually used, which can best be determined by calibration to steady state results of the experiment being simulated. 
Chapter 4

DIRECT SOLUTION OF THE PRESSURE FIELD

\subsection{Introduction}

In the solution scheme employed by NATOF-2D, the eight conservation equations, the equation of state, and the equations governing the exchange terms are reduced to a single equation for each cell which involves only the pressure of a cell and its (up to four) neighbors. The form of the equation is:

$$
a_{1 j} P_{1 j-1}+b_{1 j} P_{1-1 j}+c_{1 j} P_{1 j}+d_{1 j} P_{1+1 j}+e_{1 j} P_{1 j+1}=f_{1 j}
$$

As can be seen, the pressure of a cell is influenced only by the pressure of the cells directly in contact with it. When written out in matrix form, this large system of equations is a five-stripe band matrix, i.e. a matrix whose non-zero components are near the diagonal and contained in five bands. For example, the resulting matrix for the solution. of a problem with four axial levels and three radial nodes (figure 4.1) is shown in figure 4.2 .

Previously, NATOF-2D used an iterative solution technique known as block-tri-diagonal, which is an extension of the Gauss-Siedel iterative technique. Like all iterative methods, this scheme started from an inftial approximation and proceeded to calculate a sequence of further 


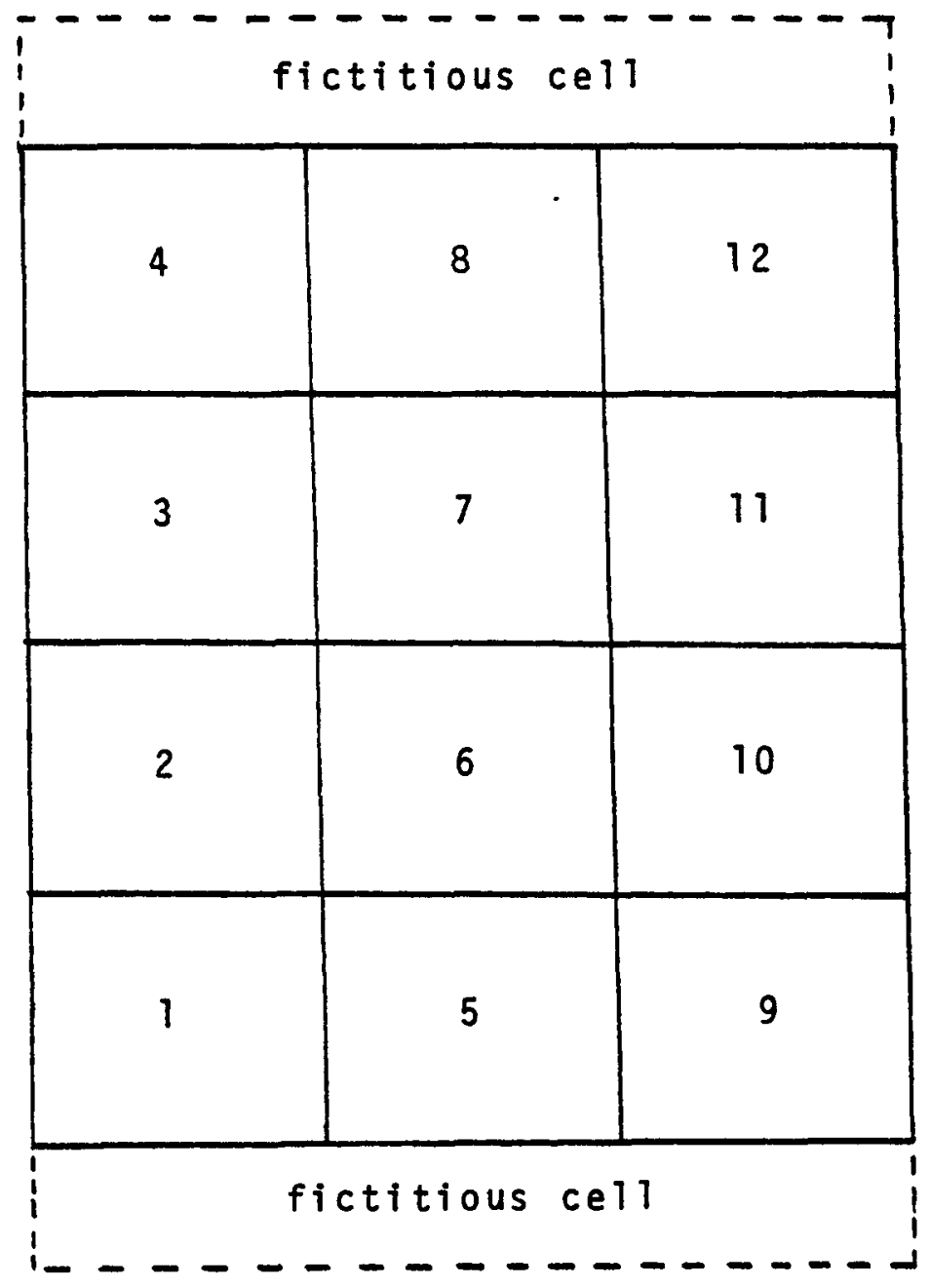

Figure 4.1 Arrangement of Cells for Pressure Field Matrix Shown in Figure 4.2 


$\left|\begin{array}{llllllllllll}a_{1,1} & a_{1,2} & 0 & 0 & a_{1,5} & 0 & 0 & 0 & 0 & 0 & 0 & 0 \\ a_{2,1} & a_{2,2} & a_{2,3} & 0 & 0 & a_{2,6} & 0 & 0 & 0 & 0 & 0 & 0 \\ 0 & a_{3,2} & a_{3,3} & a_{3,4} & 0 & 0 & a_{3,7} & 0 & 0 & 0 & 0 & 0 \\ 0 & 0 & a_{4,3} & a_{4,4} & 0 & 0 & 0 & a_{4,8} & 0 & 0 & 0 & 0 \\ a_{5,1} & 0 & 0 & 0 & a_{5,5} & a_{5,6} & 0 & 0 & a_{5,9} & 0 & 0 & 0 \\ 0 & a_{6,2} & 0 & 0 & a_{6,5} & a_{6,6} & a_{6,7} & 0 & 0 & a_{6,10} & 0 & 0 \\ 0 & 0 & a_{7,3} & 0 & 0 & a_{7,6} & a_{7,7} & a_{7,8} & 0 & 0 & a_{7,11} & 0 \\ 0 & 0 & 0 & a_{8,4} & 0 & 0 & a_{8,7} & a_{8,8} & 0 & 0 & 0 & a_{8,12} \\ 0 & 0 & 0 & 0 & a_{9,5} & 0 & 0 & 0 & a_{9,9} & a_{9,10} & 0 & 0 \\ 0 & 0 & 0 & 0 & 0 & a_{10,6} 0 & 0 & a_{10,9} & { }^{2} 10,10^{a} 10,11^{0} \\ 0 & 0 & 0 & 0 & 0 & 0 & a_{11,7} & 0 & 0 & a_{11,10^{a}} 11,11^{a_{11,12}} \\ 0 & 0 & 0 & 0 & 0 & 0 & 0 & a_{12,8} & 0 & 0 & a_{12,11} a_{12,12}\end{array}\right|$

Figure 4.2 Pressure Field Matrix 
approximations which eventually gave the required solution within a user defined convergence.

The iteration method depended on the matrix being diagonally dominant, $i . e$. the terms $b_{i j}$ and $d_{i j}$ of equation 4.1 being much smaller than the terms $a_{1 j}$ and $e_{i j}$. This situation allowed the pressure field to be solved for directly in the radial direction, and then iterations were performed in the axial direction until the solution converged. Diagonal dominance could only be maintained by having an axial mesh spacing which was much greater than the radial spacing. This limited the number of cells which could be used in simulations, and thus prevented high resolution.

Since the method employed for the pressure field solution has a strong influence on the running time of the code, a more efficient technique would result in drastically reduced costs. The method now employed is a direct method, i.e. a method which calculates the required solution without any intermediate approximations. The following section will give a background on direct methods and the solution technique employed in NATOF-2D. 
4.2 Direct Method Solution Techniques

If a matrix is of the form shown in figure 4.3 , the solution is easily accomplished by what is called "back substitution". The $n$-th equation gives $x_{n}$ directly $\left(b_{n} / u_{n}\right)$, and then the $(n-1)$ th equation can be solved for $x_{n-1}$ since $x_{n}$ is known. This procedure can be continued until $x_{1}$ is determined. In matrix terms, the system of equations Ax $=\underline{b}$ is easy to solve when $\stackrel{A}{=}$ is an upper triangular matrix. A similar situation occurs when $\stackrel{A}{=}$ is a lower triangular matrix (figure 4.4) and "forward substitution" is employed. The triangular form of the matrix can be obtained by Gaussian Elimination, for example, which uses row interchanges and addition and subtraction of multiples of rows to eliminate all terms below the diagonal. This technique can be used for small matrices, but for the large systems occurring in most NATOF-2D calculations, the computational costs become prohibitive.

In order to avoid the number of row interchanges required by Gaussian elimination, triangularization can be performed on the matrix. Triangulazization refers to factoring the matrix into a lower and a upper triangular such that

$$
\underline{\underline{A}}=\underline{\underline{L U}}
$$

where

$$
\begin{aligned}
& \underline{\underline{L}}=\text { lower triangular matrix } \\
& \underline{\underline{U}}=\text { upper triangular matrix }
\end{aligned}
$$




$$
\left|\begin{array}{llllll}
u_{11} & u_{12} & u_{13} & u_{14} & u_{15} & u_{16} \\
0 & u_{22} & u_{23} & u_{24} & u_{25} & u_{26} \\
0 & 0 & u_{33} & u_{34} & u_{35} & u_{36} \\
0 & 0 & 0 & u_{44} & u_{45} & u_{46} \\
0 & 0 & 0 & 0 & u_{55} & u_{56} \\
0 & 0 & 0 & 0 & 0 & u_{66}
\end{array}\right| \times\left|\begin{array}{c}
x_{1} \\
x_{2} \\
x_{3} \\
x_{4} \\
x_{5} \\
x_{6}
\end{array}\right|=\left|\begin{array}{c}
b_{1} \\
b_{2} \\
b_{3} \\
b_{4} \\
b_{5} \\
b_{6}
\end{array}\right|
$$

Figure 4.3 Upper Triangular Matrix

$$
\left|\begin{array}{llllll}
l_{11} & 0 & 0 & 0 & 0 & 0 \\
l_{21} & l_{22} & 0 & 0 & 0 & 0 \\
l_{31} & l_{32} & l_{33} & 0 & 0 & 0 \\
l_{41} & 1_{42} & l_{43} & l_{44} & 0 & 0 \\
l_{51} & l_{52} & l_{53} & l_{54} & l_{55} & 0 \\
l_{61} & l_{62} & l_{63} & l_{64} & l_{65} & l_{66}
\end{array}\right| \times\left|\begin{array}{l}
x_{1} \\
x_{2} \\
x_{3} \\
x_{4} \\
x_{5} \\
x_{6}
\end{array}\right|=\left|\begin{array}{l}
b_{1} \\
b_{2} \\
b_{3} \\
b_{4} \\
b_{5} \\
b_{6}
\end{array}\right|
$$

Figure 4.4 Lower Triangular Matrix 
Thus the system of equations

$$
\underline{\underline{A}} \underline{\underline{x}}=\underline{\mathrm{b}}
$$

becomes

$$
\underline{\underline{L}} \underline{\underline{\underline{x}}}=\underline{\mathrm{b}}
$$

The factorization, when possible, is unique. / $10 /$ Defining $\underline{y}=\underline{\underline{U}} x$, the system $\underline{\underline{L}} \underline{\underline{y}}=\underline{\underline{b}}$ can be solved for $\underline{\mathrm{y}}$ by forward substitution. Then, $\underline{\underline{U}} \underline{x}=\underline{y}$ can be solved for $x$ by backward substitution. This is the basic technique used for the direct solution of the pressure field employed by NATOF-2D.

Since the pressure field matrix is in band form, the number of operations required for the LU factorization is reduced due to the large number of zeros. This is especially true if the bandwidth is much less than the dimension of the matrix. The bandwidth of a matrix $A$ has a value $w$ if $a_{1 j}=0$ whenever $|i-j| \geq w$. For example, the matrix in figure 4.2 has a bandwidth of 5 .

Taking into account the presence of the zeros, the terms of the upper triangular matrix are given by $/$ II /:

$$
\begin{aligned}
u_{i j}=a_{i j}- & \sum_{k}^{1-1} \ell_{i k} u_{k j} \\
k & =\max (1, j-w+1)
\end{aligned}
$$

where

$$
\begin{aligned}
& j=i, \ldots \min (i+w-1, n) \\
& n=\operatorname{dimension} \text { of the matrix } \underline{A}
\end{aligned}
$$


The terms of the lower triangular matrix are given by the relation:

$$
\begin{aligned}
& \ell_{i j}=u_{j j}^{-1}\left(a_{i j}-l u_{k j} \ell_{i k}\right) \\
& k=\max (1, i-w+1)
\end{aligned}
$$

where

$$
i=j+1, \ldots \min (j+1-w, n)
$$

A comparison between the count of operations using Gaussian elimination and using a LU factorization of a band matrix is given by Franklin / $11 /$. For Gaussian elimination there are:

$$
\begin{array}{ll}
c_{1}=n^{2}+(n-1) n(n+1) / 3 & \begin{array}{c}
\text { multiplications or } \\
\text { divisions }
\end{array} \\
c_{2}=n(n-1)+(n-1) n(2 n+1) / 6 & \begin{array}{c}
\text { additions or } \\
\text { subtractions }
\end{array}
\end{array}
$$

For the LU factorization and solution there are

$$
\begin{aligned}
& c_{1}=w(w-1)(3 n-2 w+1) / 3+(2 w-1) n-w(w-1) \\
& \text { multiplications or } \\
& \text { divisions } \\
& c_{2}=w(w-1)(3 n-2 w+1) / 3 \\
& \text { additions or } \\
& \text { subtractions }
\end{aligned}
$$


For the matrix given in figure 4.2 the count would be

Gaussian Elimination

LU Factorization and Solution

$\begin{array}{rr}\frac{c_{1}}{1616} & \frac{C_{2}}{1480} \\ 384 & 260\end{array}$

260

As can be seen, the saving is substantial.

The above result shows that the number of operations required for the LU factorization and solution has a strong dependence on the bandwidth of the matrix. The bandwidth can be drastically reduced by reordering the numbering of the cells. In NATOF-2D, the numbering scheme is to count from the bottom to the top for each cell in a channel. Thus a problem with twelve axial levels and three radial nodes has a bandwidth of thirteen. However, by rearranging the numbering so that the cells are numbered across for each axial level, the bandwidth is reduced to four.

Since there will be a number of divisions by the diagonal elements, a partial pivoting strategy is also employed to reduce cumulative rounding error. This is achieved by reordering the rows of the matrix such that the largest elements appear on the diagonal. / 12 / 
4.3 A Comparison of Direct and Iterative Methods in NATOF-2D

In this section a comparison is made between the Central Processing Unit (CPU) time usage of the direct and iterative solution methods for both steady state and transient calculations. The test case used for the comparison had 12 axial levels and 5 radial nodes, which gave a matrix of typical size for most NATOF-2D calculations. The results are plotted as CPU time versus time into the simulation.

For the steady state calculation, shown in figure 4.5, the CPU usage for the direct solution is about 100 seconds less than for the iterative solution. The major difference in CPU usage occurs at the start of the calculation, when the system is settling down. As the time into the calculation increases, the CPU usage per Newton iteration decreases for the iterative solution. This is to be expected, since the change in pressure per time step is converging to zero, and therefore fewer iterations are required to meet the convergence criterion. For steady state calculations of a longer duration, the iterative solution may in fact be quicker, since the direct method takes a fixed amount of time to solve the pressure field matrix regardless of the pressure change increment.

For the single phase transient case, shown in figure 4.6, the direct solution used only half the CPU time of the 


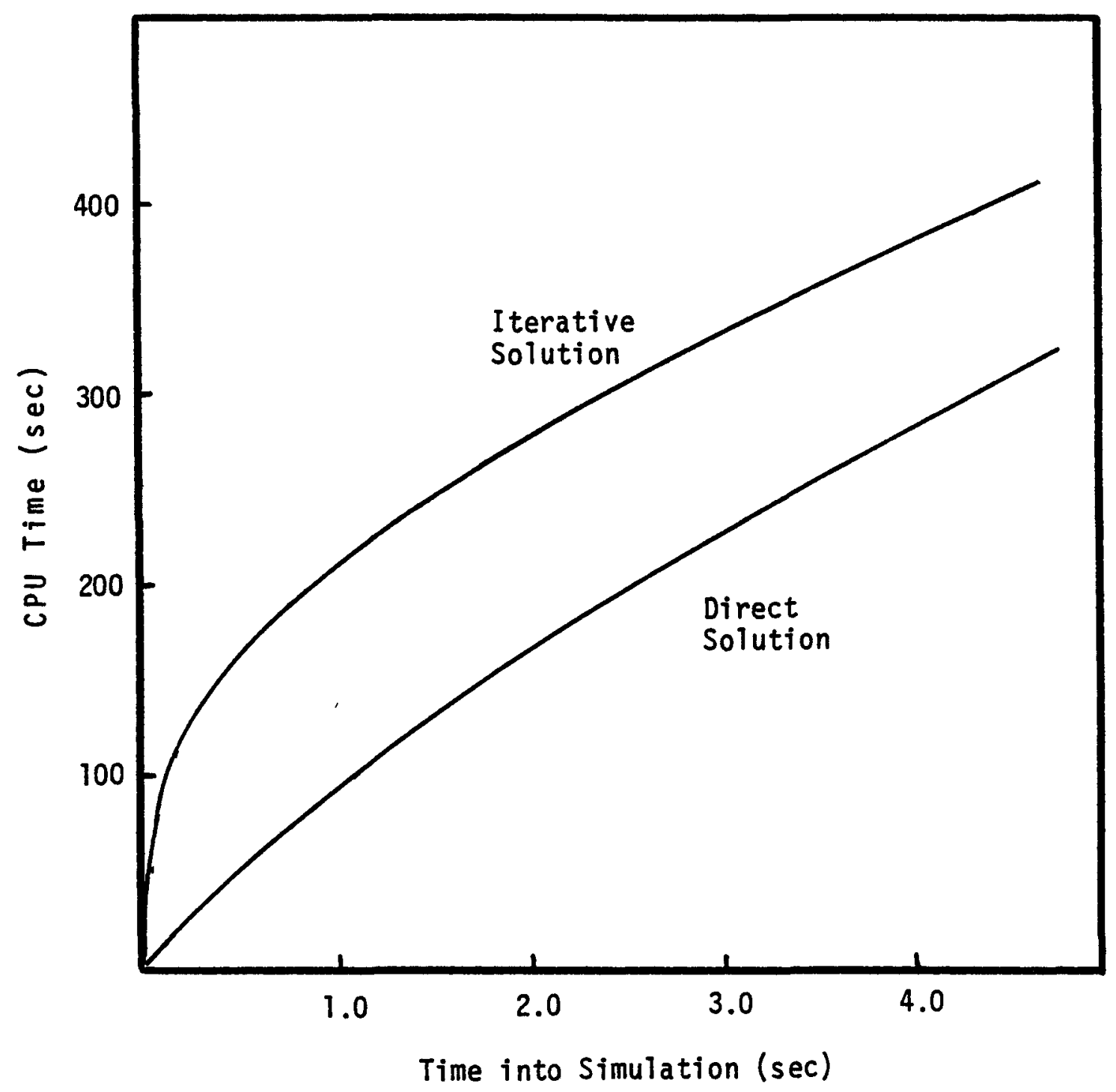

Figure 4.5 A Comparison of Steady State CPU Usage Between the Direct and I terative Techniques (10 Axial Levels, 5 Radial Nodes) 


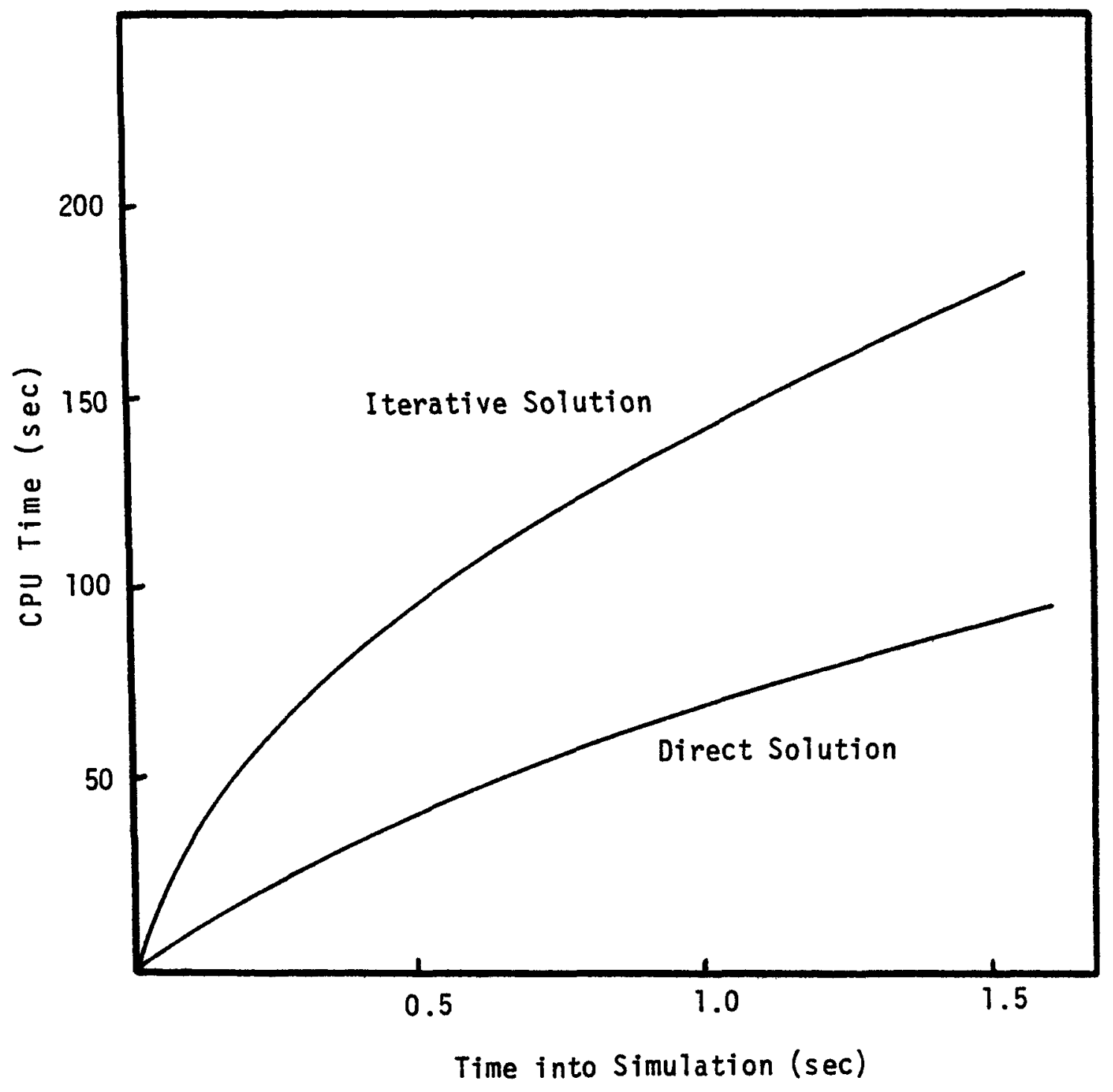

Figure 4.6 A Comparison of Transient CPU Usage Between the Direct and Iterative Techniques

(10 Axial Levels, 5 Radial Nodes) 
$-100-$

iterative solution. This is a considerable savings when the CPU requirements are large. Experience has shown that during boiling transients, the large pressure changes cause the iterative solution to have an even greater CPU time usage relative to the direct method.

A transient was also run with a decreased mesh spacing, so that there were now 42 cells in the axial direction, covering approximately the same length as before. The iterative technique took four times as much CPU time as the direct solution, thus demonstrating the important role diagonal dominance plays in the iterative technique. 
4.4 Programming Information

Two subroutines have been incorporated into NATOF-2D to perform the direct solution. The first one is subroutine DIRECT which rearranges the pressure field matrix in order to minimize the bandwidth. The second is subroutine LEQTIB which performs the direct solution. Subroutine LEQT1B is a commercial subroutine available on the MULTICS computer system at M.I.T. which performs Lower-Upper Factorization and the solution of Band Matrices. The basic algorithum of this subroutine can be found in / $10 /$. Due to copyright laws, this subroutine cannot be disseminated outside of the Massachusetts Institute of Technology. However, a subroutine of similar form should be available on most computer systems.

The user has the option to specify either a direct or iterative solution technique by setting the input parameter indgs to either 0 or 1 respectively. 
Chapter 5

EXPERIENCES WITH NATOF-2D

\subsection{Introduction}

The original development of NATOF-2D wasn't completed until June of 1980, and therefore prior experience with running the code was of a limited nature. However, the extremely low computational cost avallable at M.I.T. over the past six months have allowed numerous testing simulations to be made which yielded information in the area of code capability and constraints. In this chapter some of the experiences encountered will be documented in order to provide a foundation for future work, and also provide an explanation for any changes made to the constituative relations. 
5.2 Double versus Single Precision

In an effort to reduce both memory storage and computational costs, NATOF-2D was converted to a single precision code. Single precision refers to the number of significant digits in which a variable is stored and to which a variable is computed. On MULTICS there are 9 significant digits in single precision, and 18 in double precision. At the time, it was felt that carrying out calculations to the 18 th place was being excessive.

A comparison was made between the results of the same single phase transient computed in both single and double precision. The results showed exact agreement in the value of variables up to the eigth significant digit. This was encouraging since the single precision computational costs were $25 \%$ less.

However, in two phase boiling transients where the timestep size was considerably smaller, problems were encountered in obtaining convergence of the Newton Iterations for the single precision version of NATOF-2D. To explore this problem, the code was modified so that the user could impose a series of decreasing time step sizes on a calculation. The procedure was to allow a single phase calculation to reach a steady state solution, and then gradually reduce the timestep size. Since the code was already at a steady state solution, convergence should always be attained. This is particularly true for the 
iteration scheme used, since as $\Delta t \rightarrow 0, \Delta P \rightarrow 0$. However, the results showed that for small timestep sizes, the code actually diverged, and had difficulty in reaching the convergence criteria (in NATOF-2D, convergence is assumed if $\delta \mathrm{P}_{\max }$ ( user input). If the convergence criteria was relaxed, the code could run to slightly smaller timestep sizes, but it still wasn't converging on zero as a steady state solution implies. Table 5.1 lists the smallest timestep size for various convergence criterias.

Table 5.1

$\begin{array}{cc}\text { Newton Iteration Convergence } & \text { Minimum } \text { Timestep Size } \\ \left(\mathrm{N} / \mathrm{m}^{2}\right) & (\text { seconds }) \\ 0.01 & 10^{-2} \\ 0.1 & 10^{-3} \\ 1.0 & 10^{-4} \\ 10.0 & 10^{-6}\end{array}$

The problem experienced was traced to the energy conservation equation. The solution scheme employed requires that the quantity

$$
\frac{\left((1-\alpha) \rho_{\ell} e_{\ell}\right)^{n+1}-\left((1-\alpha) \rho_{\ell} e_{\ell}\right)^{n}}{\Delta t}
$$


be evaluated at each timestep. For small timesteps sizes and/or in a steady state configuration, the term $\rho_{\ell} e_{\ell}$ has a very small variation. However, the magnitude of this term is typically of the order of $10^{9}$, which is the same as the machine precision. In any single precision computation, it is reasonable to assume that the 1 st 8 digits are valid, but that the 9 th digit is subject to "noise" fluctuations and computational roundoff errors. Yet for small timesteps, the calculation was relying on this term to solve the equation. Any error would then be inversely proportional to the timestep size.

When the code was returned to double precision, timesteps of $10^{-10}$ seconds could be attained with no stability problems. From this experience it has been concluded that double precision is a necessity for NATOF-2D. 
5.3 On the Modelling of Sodium Reactors

Numerous problems have been encountered in the phenomenological modelling of sodium boiling transients which were not encountered in PWR and BWR modelling. The reason for this difficulty can be traced to the characteristics of LMFBRs and the properties of sodium. A comparison between typical PWR and LMFBR characteristics, and water and sodium properties is given in Table 5.2 and Table 5.3 respectively.

As shown in Table 5.2 , the most striking difference in core properties is the temperature rise of the coolant per unit length. For a PWR this is $9.7^{\circ} \mathrm{C} / \mathrm{m}$, while for an LMFBR it is $125.4^{\circ} \mathrm{C} / \mathrm{m}$. In the numerical scheme employed in NATOF-2D, the core is divided into a finite number of axial levels. Unless the number is large, there will always exist a substantial temperature difference from cell to cell. In some of the loss-of-flow transients simulated, this can be as much as $150^{\circ} \mathrm{C}$. Combined with the higher power density, a model for LMFBR transient analysis experiences rapid temperature changes throughout its length not experienced by PWR codes.

The second major difference is the density ratio, $\rho_{\ell} / \rho_{V}$, which is approximately 6 for water at 2200 psi, and 1000 for sodium at 44 psi. At atmospheric pressure, the ratio for sodium increases to 3000 . The large density ratio for sodium leads to a rapid voiding of the 
A Comparison of PWR and LMFBR properties / 13 /

Proposed

\begin{tabular}{lll} 
& \multicolumn{1}{c}{ PWR } & LMFBR \\
Core Thermal Power (MWth) & 3,411 & 3,800 \\
Core Diameter (m) & 3.4 & 3.11 \\
Core Height (m) & 3.7 & 1.22 \\
Core Power Density (kw/liter) & 98 & 395.7 \\
Reactor Inlet Temperature $\left({ }^{\circ} \mathrm{C}\right)$ & 289 & 385 \\
Reactor Outlet Temperature $\left({ }^{\circ} \mathrm{C}\right)$ & 325 & 538 \\
System Flow Rate (total $\left.10^{6} \mathrm{Ib} / \mathrm{hr}\right)$ & 136 & 136.8
\end{tabular}

Table 5.3

A comparison of Water and Sodium Properties / $14 /$

$\begin{array}{lcc} & \text { Water } & \text { Sodium } \\ \text { Pressure (psi) } & 2250 & 44 \\ \text { Saturation Temperature }\left({ }^{\circ} \mathrm{C}\right) & 346 . & 1016 . \\ \text { Liquid Density }\left(\mathrm{kg} / \mathrm{m}^{3}\right) & 593.4 & 707.5 \\ \text { Vapor Density }(\mathrm{kg} / \mathrm{m} 3) & 102 . & 0.748 \\ \text { Liquid Specific Heat }\left(\mathrm{J} / \mathrm{kg}-{ }^{\circ} \mathrm{K}\right) & 9211 . & 1324 . \\ \text { Liquid Conductivity }\left(\mathrm{W} / \mathrm{m}-{ }^{\circ} \mathrm{K}\right) & 0.4074 & 46.03 \\ \text { Vapor Specific Heat }\left(\mathrm{J} / \mathrm{kg}-{ }^{\circ} \mathrm{K}\right) & 7709.4 & 281.8 \\ \text { Vapor Conductivity }\left(\mathrm{W} / \mathrm{m}-{ }^{\circ} \mathrm{K}\right) & 0.1061 & .073\end{array}$


core for extremely small superheats. Accompanying this phenomenon is the expulsion of the liquid phase, and a mass depletion of the core often resulting in flow reversal. The numerical difficulty experienced during the initial stages of boiling is attributable to the fact that the density of the cell varies by a factor of 1000 in a very short timespan.

The boling transients which the code is required to simulate are thus of an extremely harsh nature. For these reasons, the mass exchange rate plays a critical role in the calculations. 
5.4 The Mass Exchange Rate

of all the constituative relations used in NATOF-2D, the mass exchange rate is probably the most important. The basic physical requirements of any mass exchange model is that the vapor production rate should not exceed the limit established by equilibrium, and also that it should prevent a two-phase situation with highly superheated liquid or subcooled vapor. In essence, it should tend toward equilibrium.

The mass exchange rate determines the rate of vapor evaporation and condensation. The high power density of the core and the high density ratio leads to void fractions of 0.9 in as little as $1 / 10$ th of a second. As the void travels into subcooled liquid regions, it is required to condense quickly, since it contains a negligible amount of energy. However, unlike cells where evaporation is occurring and thus the mixture density remains low throughout the transient, cells where condensation is occurring are required to experience rapid density changes throughout the transient.

Since at even void fractions of 0.95 , the vapor phase represents only $2 \%$ of the mass in the cell, rapid condensation requires that either the pressure of the cell decrease (in order to lower the saturation temperature) or else that the mass flux into the cell be extremely large. The requirements of a large condensation rate have often 
lead to code failure on a negative cell pressure error. In either case, the change in pressure for the timestep is large.

The pressure of a cell is the key variable in the numerical scheme of NATOF-2D. Large variations in pressure thus effect the stability of the code, since it is the change in pressure, $\delta P$, of a Newton iteration, which determines if convergence has occurred. Defining the convergence criteria in relative terms such that

$$
\text { convergence }=\frac{\delta P}{P}
$$

a convergence of $10^{-10}$ can easily be attained in single phase calculations. However, in boiling transients, the convergence must be relaxed to $10^{-3}$ or $10^{-4}$. This has proven a necessity if timesteps are to be taken which are within computational time limitations (1.e. $10^{-3}$ or $10^{-4}$ sec)

Small timestep sizes, well below the convective limit, are necessary, since the effect of reducing the timestep size is to reduce the magnitude of $\Gamma$, which has units of $\mathrm{kg} / \mathrm{s}-\mathrm{m}$ ? One noticeable phenomenon during sodium boiling siumlations, is the appearance of a stable boiling timestep, or SBT. The SBT refers to the timestep size at which the code can attain a reasonable convergence $\left(10^{-3}-10^{-4}\right)$ in a single Newton Iteration, but above which convergence cannot be obtained regardless of the number of Newton Iterations. 
The SBT appears to be a function of the convergence criteria, the mesh cell spacing distance (whose effects are covered in the next section) and the condensation rate.

The condensation rate, as previously mentioned, has a profound effect, since it requires a large density change corresponding to a small energy change. By numerically reducing the rate (i.e. multiplying it by a small number, which will be designated $(F)$, the calculation proceeds more quickly. What essentially occurs is that the code is. allowed to operate in a highly nonequilibrium, two-phase low density mode which prevents the need to make the large density change. The effect is so pronounced that setting the condensation rate to zero allows the code to run at a timestep not limited by any boiling effects (except during the short period of boiling inception) but by the convective limit $\left(10^{-2} \mathrm{sec}\right.$ due to the high vapor velocities), while setting the multiplicative factor to one necessitates timestep sizes of $10^{-7}$ seconds. In the past, this manipulation has been justified by reference to experiments whlch showed the condensation rate to be slightly lower than the evaporation rate. However, the fact that analytic results done with NATOF-2D show vapor coexisting with liquid subcooled by hundreds of degrees negates this premise. However, in the experiments simulated in this work, and covered in chapter 6 , this manipulation has been necessary in order to obtain results of a time spanning any reasonable 
duration.

In the previous formulation used for the mass exchange rate, the term $\alpha(1-\alpha)$ was added. This term had the effect of inhibiting condensation at small void fractions, and thus allowed the code to run smoother than the formulation presently implimented, which requires higher condensation. 


\subsection{Varying Mesh Spacings}

In order to minimize the effects of the large temperature gradients in the axial direction, the number of axial cells was increased. The two advantags of this are that by decreasing $\Delta T$ from cell to cell, the mass exchange rate decreases, and also the results are of a more detailed nature. Smaller mesh cells reduce the inaccuracies caused by volume averaging of the fluid properties. As mentioned in Chapter 4, NATOF-2D could not use small mesh spacing due to the necessity of maintaining diagonal dominance in order to obtain the pressure field solution. However, the direct solution technique does not have this constraint, and so small mesh spacing becomes possible.

There is a practical limit to the number of axial levels. For example, if the number of levels is increased by a factor of 10 , the number of computational steps per Newton Iteration will be roughly ten times as much. However, the convective time limit, $\Delta z / v$, would decrease the time step size also by a factor of ten, so that the computation would need about 100 times more cpu time to compute the same time length in single phase. It is doubtful that a time step size two orders of magnitude greater than before could be taken during the boiling transient.

For testing purposes, the number of axial levels was increased by a factor of 4 , from 10 levels to 40 levels, to 
see whether any increased timesteps could be taken during boiling. The decreased mesh spacing allowed timesteps to be taken which varied from $2 \times 10^{-3}$ seconds to $10^{-4}$ seconds, with a condensation factor of $=0.01$. The cpu usage was roughly three times greater than for the 10 axial level case, which had a $C F=0.002$. Increasing $C F$ to 0.1 , for the same 40 axial level calculation resulted in a cpu usage 6 times greater, and a SBT of $2 \times 10^{-4}$ seconds.

The conclusion drawn from this is that higher accuracy results can be attained without the corresponding cpu costs by decreasing the mesh spacing. For the experimental test simulations covered in Chapter 6,40 axial levels were used. 
Chapter 6

VERIFICATION OF MODELS

\subsection{Introduction}

In order to test the capability of NATOF-2D and the validity of the models described in the previous chapters, a total of five sodium boiling transients were simulated. The first one was THORS Bundle $6 \mathrm{~A}$ Run 101 conducted at Oak Ridge National Laboratory / 4 /. The other four were from the W-1 SLSF Experiments, done by the Hanford Engineering Development Laboratory, and include two Loss of Piping Integrity transients and two Boiling Window Tests / $5 /$.

The tests cover a wide range of conditions under which the code will be required to operate. In contrast to previous simulations / $1 /$, the decision has been made to drastically increase the number of mesh cells. This improved the quality of the results, but also constrained the length of the calculation due to the large CPU usage.

In the next sections, a description of the runs will be given, and the results will be presented. 
6.2 THORS Bundle 6A Experiment, Test $71 \mathrm{H}$ Run 101

6.2.1 Description of the THORS Bundle 6A Experiments The purpose of the THORS Bundle 6A Experiments / 4 / was to investigate the extent of dynamic boiling stability at low flow conditions. The tests were conducted in the THORS Facility, an engineering-scale high-temperature sodium facility for the thermo-hydraulic testing of LMFBR subassembiies.

The test section used was a full-length simulated LMFBR fuel subassembly. It consisted of 19 electrically heated fuel pin simulator units spaced by helical wire-wrap spacers. The heated length of Bundle $6 \mathrm{~A}$ was 0.9 meters, and had variable pitch heater windings to produce a 1.3 axial peak-to-mean chopped cosine power distribution.

Appreciable effort was expended in designing and fabricating a low thermal inertia bundle housing. However, a posttest analysis revealed that sodium had penetrated the entire housing region, and thus the housing had a high heat capacity.

The selected run from this series was Test $71 \mathrm{H}$, Run 101. The bundle power level was $127 \mathrm{kw}$. This was the lowest power run that exhibited dryout. At 3.2 seconds into the transient, the initial flow of $0.39 \mathrm{l} / \mathrm{s}$ was decreased over a period of 3.6 seconds to a flow of $0.12 \mathrm{l} / \mathrm{s}$. Boiling inception occurred at 13.7 seconds. Flow oscillations 
occurred at a frequency of 1.1 to $1.5 \mathrm{~Hz}$. A geometry description of the bundle is given in Table 6.1. In Figure 6.1 the locations of the axial levels used in the NATOF-2D simulation are shown.

The simulation was carried out under inlet velocity, outlet pressure boundary conditions during the single phase part of the transient. This allowed the exact flow rate to be maintained, while the large number of axial cells permitted the proper flow splits to be established in the first three or four cells. At the point of boiling inception, the boundary conditions were switched to pressure/pressure, and the transient was continued.

For this simulation, $C F$ was 0.01 , and the fluid-to-fluid radial heat conduction nusselt numbers were $N u_{1}=13$ and $N u_{2}=13$. The input parameters for the simulation are given in Appendix C.3.

\subsubsection{Simulation Results}

The time of boiling inception during the NATOF-2D simulation was approximately 2.7 seconds sooner than the THORS experiment. This can be attributed to underestimating the heat capacity of the hexcan. In NATOF-2D, the hexcan is modelled as a heat capacitance. During transients the hexcan is heated up and cooled off by the coolant, with no losses to the environment. In principal, by adjusting the heat capacity of the hexcan, the boiling inception time can 
Description of THORS Bundle $6 \mathrm{~A}$

Number of Pins

Pin Diameter ( $m$ )

Pitch/Diameter Ratio

Wire Wrap Diameter $(\mathrm{m})$

Distance from fuel pin to the wall (m)

Heated Length $(\mathrm{m})$

Axial Power Distribution, Cosine

$$
\text { peak/mean }
$$

Radial Power Distribution, peak/mean

Total Bundle Power (kw)

Pin Power (kw/pin)

Inlet Flow, Steady State $(\mathrm{kg} / \mathrm{s})$

Inlet Flow, Boiling Inception ( $\mathrm{kg} / \mathrm{s})$

Inlet Liquid Temperature $\left({ }^{\circ} \mathrm{K}\right)$
19

$$
5.84 \times 10^{-3}
$$

$1.42 \times 10^{-3}$

$0.71 \times 10^{-3}$

0.9

1.3

1.0

127

6.7

0.3344

0.1029

660.91 
Height (m)

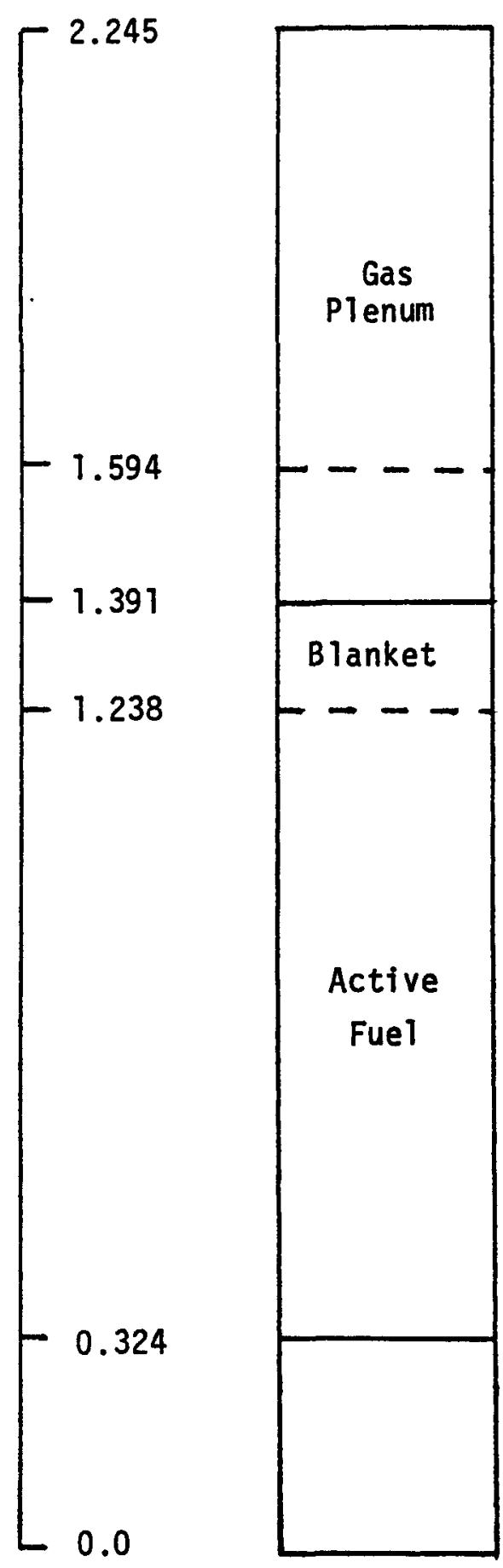

Axial

Ce11 Leve1

40

31

27

24

6

Figure 6.1 Location of Cells used in the NATOF-2D Simulation of THORS Bundle 6A Experiment 
assume any desired value. Prior to the start of a calculation, the value is estimated based on the properties of the can. The results of this test indicate the need for a more sophisticated model. Appendix $D$ discusses the sensitivity of the boiling inception time to the hexcan heat capacity.

At the time of boiling inception, flow reversal followed almost immediately. There are two reasons for this. The first is that the small rate of condensation allows the channel to void rapidly, which increases the fluid pressure and forms a flow blockage. The second reason is that information about pressure drops at the inlet due to valve adjustments was not documented, so this effect could not be accurately simulated. As with the hexcan heat capacity, it is possible to adjust the spacer pressure drop feature of NATOF-2D to obtain the experiment's flow reversal time. Appendix $E$ gives typical values of the spacer pressure drop, and its effect on flow reversal.

Figure 6.2 shows the inlet mass flow rate during the transient. The flow oscillations were quite severe and had a frequency of about $3 \mathrm{~Hz}$. This was about twice the frequency of oscillations found in the experiment. In Figure 6.3 , the temperature profile of the central channel at various points of time during the transient is plotted. Figure 6.4 shows the temperature history at the end of the heated section for both the central and edge channels. This 

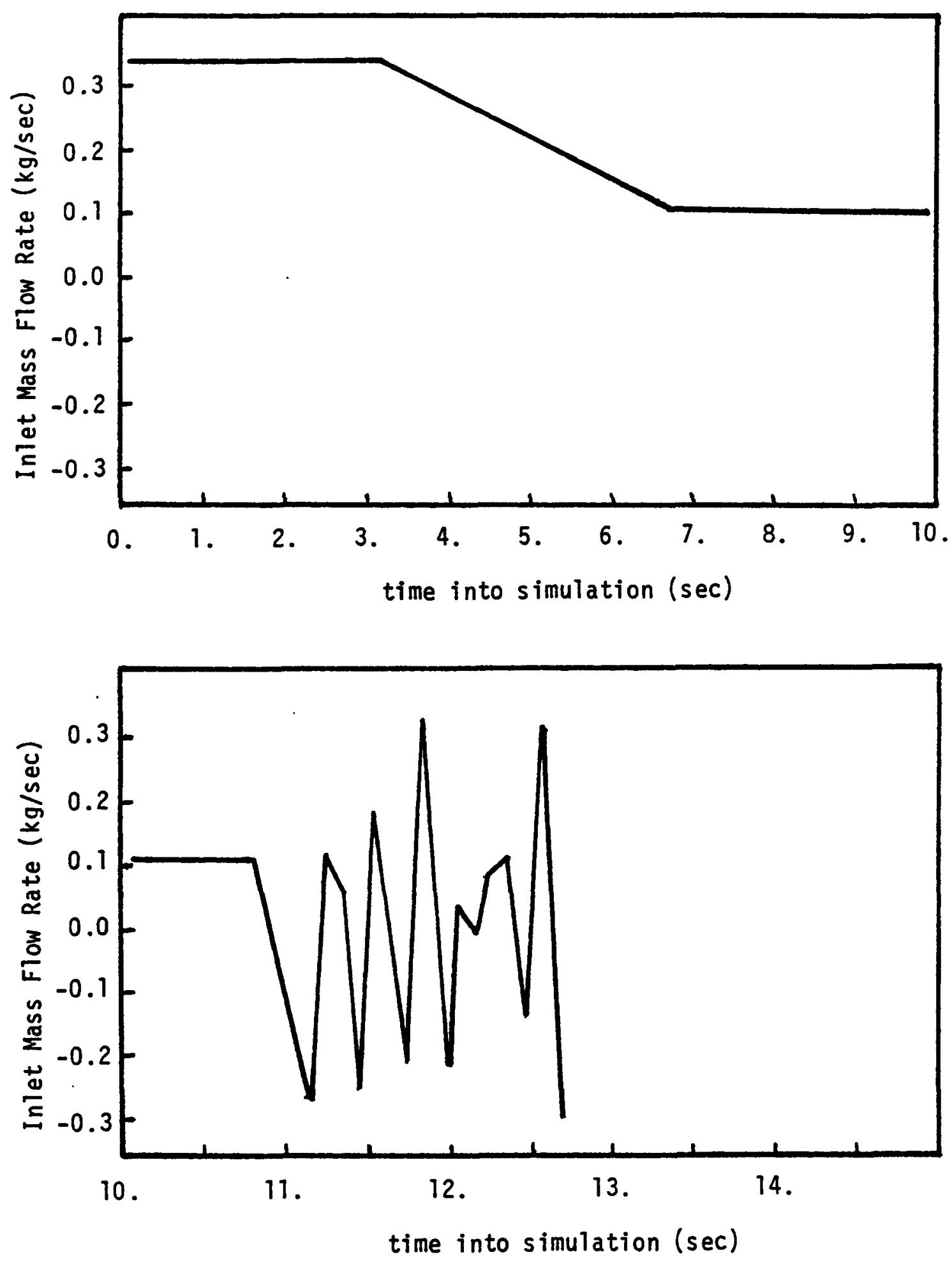

Figure 6.2 NATOF-2D Predicted Inlet Mass Flow Rate of THORS Bundle 6 A Test $71 \mathrm{H}$, Run 101 


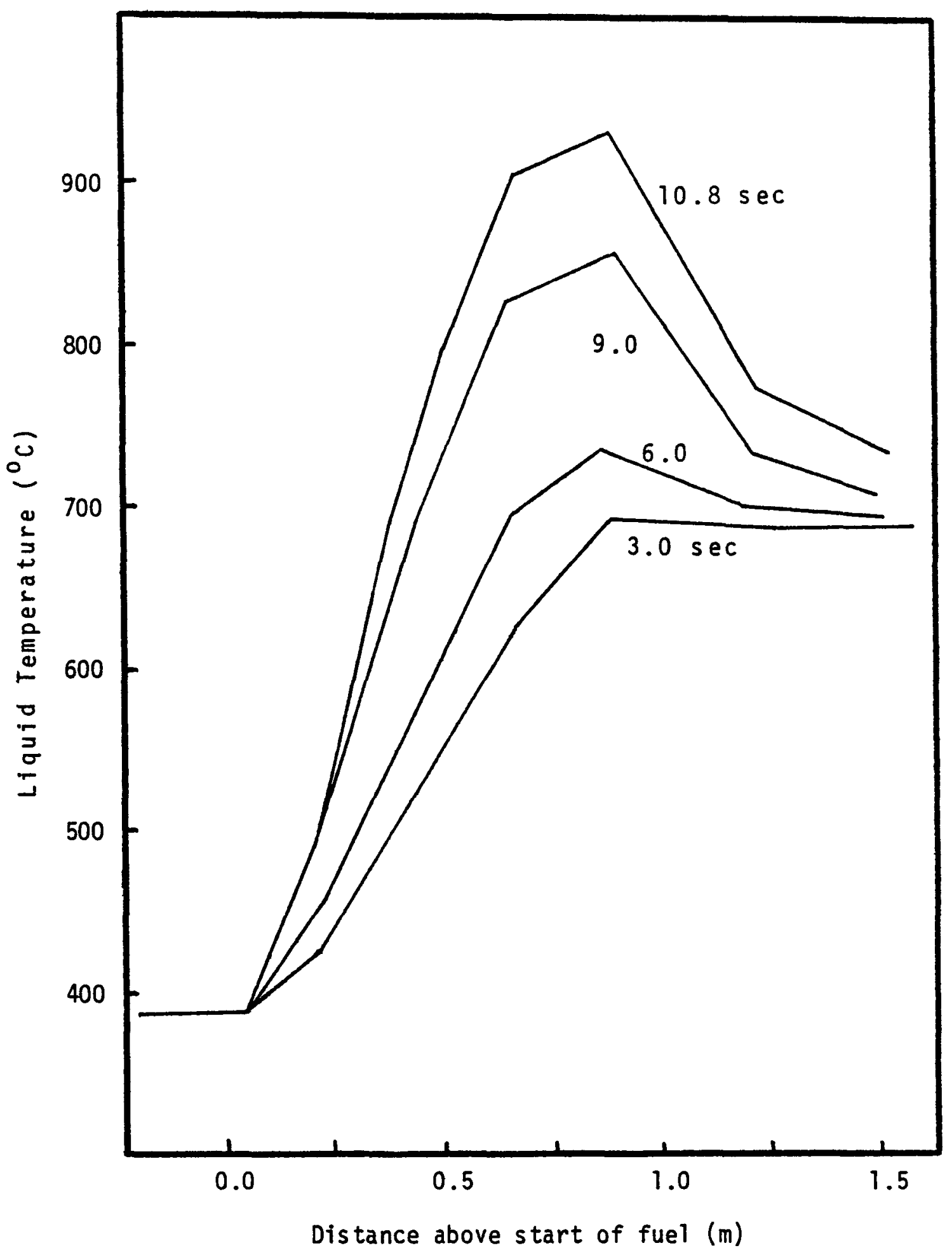

Figure 6.3 NATOF-2D Temperature Profiles of Central Channel at Various Points in Time (THORS Bundle 6A Test 71H, Run 101) 


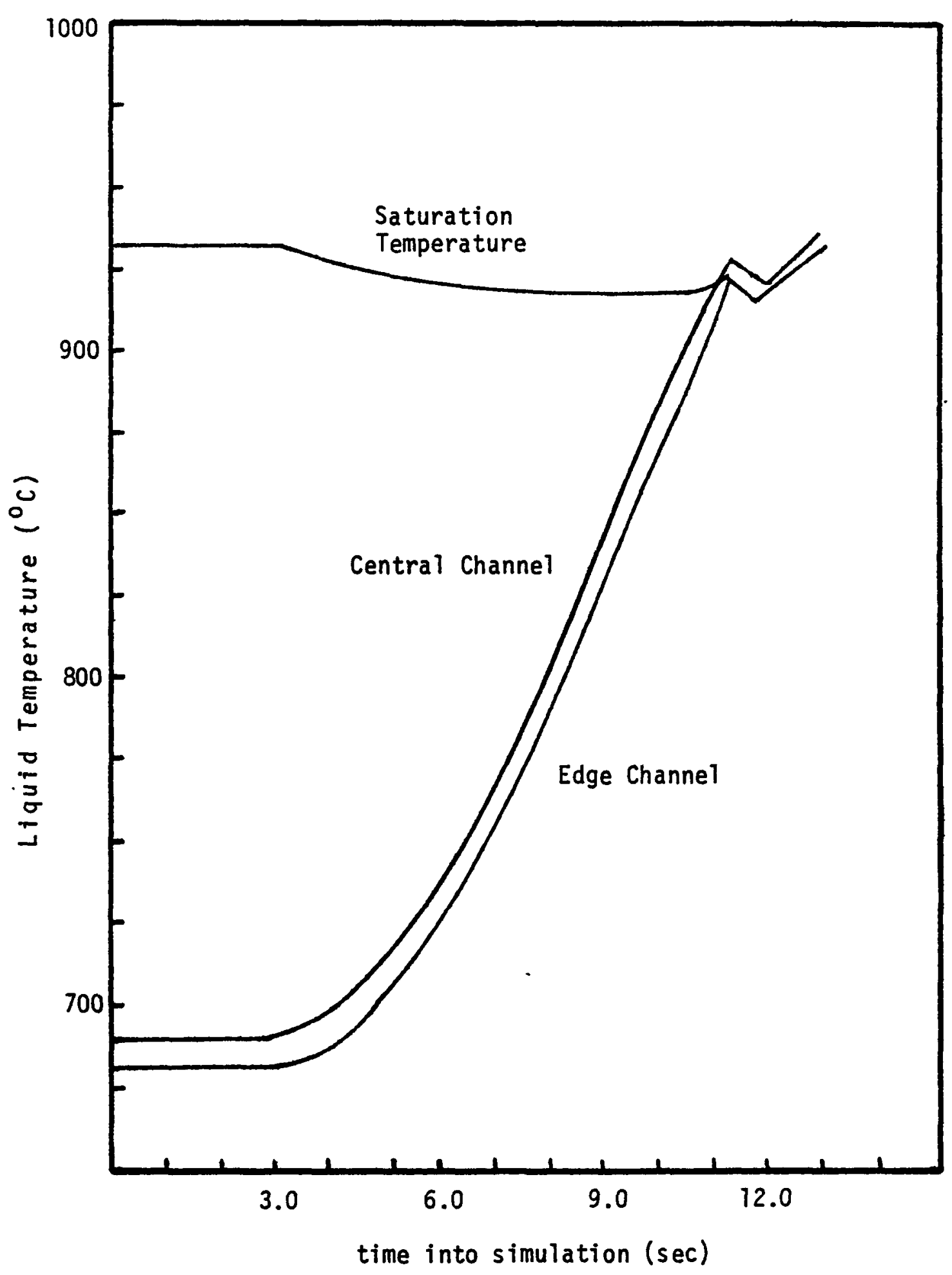

Figure 6.4 NATOF-2D Predicted Temperature Histories at End of the Heated Zone for the Central and Edge Channels (THORS Bundle 6A Test 71H, Run 101) 


\section{$-124-$}

figure demonstrates the effects of the radial heat conduction model in reducing radial temperature variations to levels comparable with the test results.

Figure 6.5 compares the vapor and liquid velocities. The large difference in velocities indicates the need for a separated flow model. 


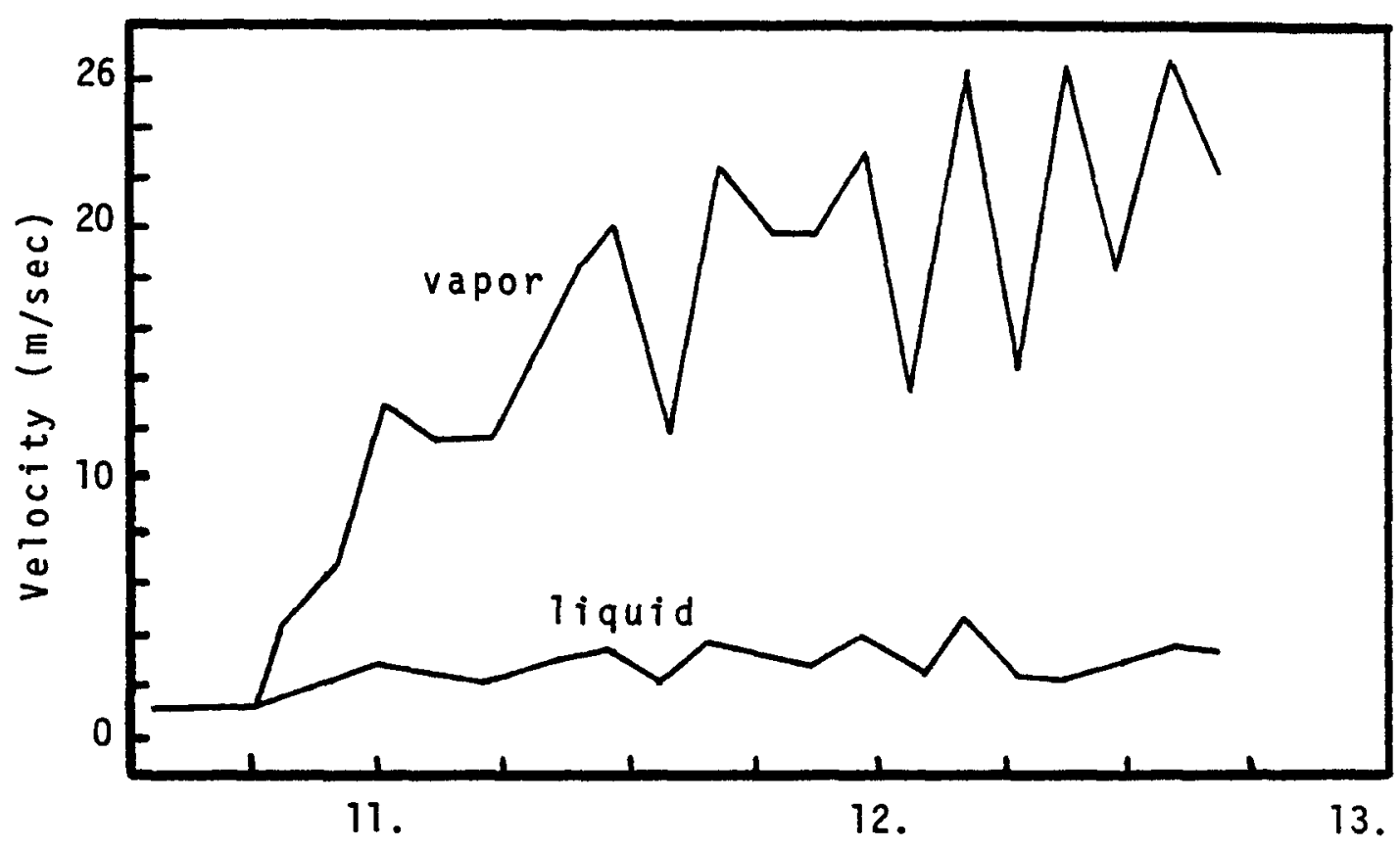

time into transient (sec) -- End of the Heated Zone

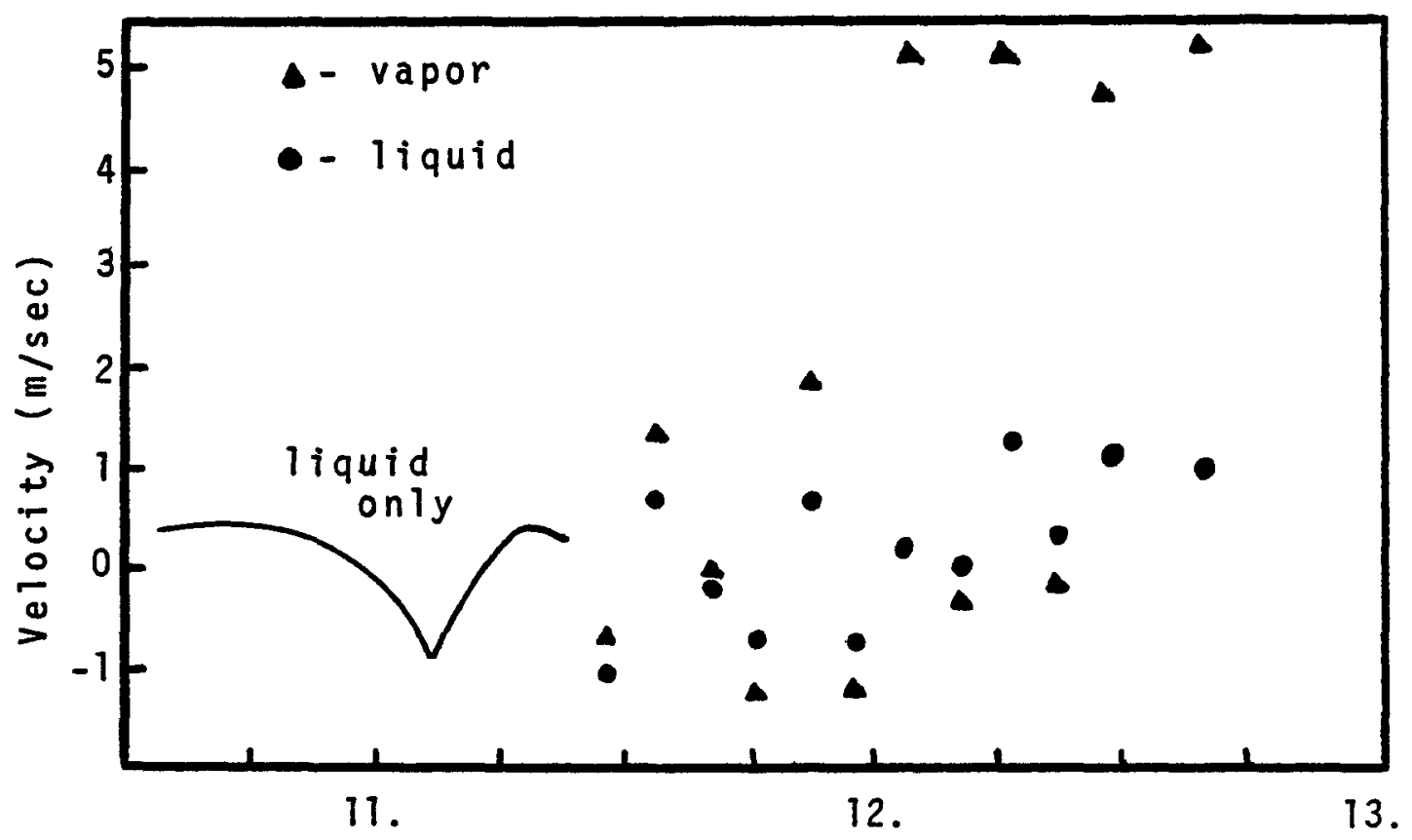

time into transient (sec) -- Midplane of Heated Zone

Figure 6.5 A Comparison of NATOF-2D Predicted Liquid and Vapor Velocities

(THORS Bundle $6 \mathrm{~A}$ Test $71 \mathrm{H}$, Run 101) 


\subsection{The $W-1$ SLSF Experiments}

\subsubsection{Test Objective}

The W-1 SLSF Experiment / $5 /$ was designed to provide experimental data on sodium bolling and boiling "stability" in a fuel pin bundle during flow transients under LMFBR accident conditions. The test was divided into two groups. The first was the Loss-of-Piping Integrity (LOPI) accident simulation. The objective of this series was to determine the heat transfer characteristics from fuel pins to sodium coolant during a transient simulating a double ended pipe break at the primary vessel inlet nozzle. The difference in heat transfer characteristics as a result of fuel conditions were studied.

The second group of tests was the Boiling Window Tests. The objective of this series was to establish the family of flow/heat-flux combinations that will produce incipient boiling in the bundle for a given inlet temperature. The test runs were designed to determine whether or not there is a regime of boiling beyond the onset that persists and does not immediately lead to dryout for low flow and intermediate to high heat fluxes.

The SLSF $W-1$ boiling window tests were conducted in three operating phases: approach to boiling, incipient boiling and dryout with fuel pin failure. The incipient boiling tests were designed to determine low heat flux 
combinations for which the onset of sodium boiling is achieved. One dryout test was performed to identify the far end of the "boiling window" at the highest heat flux tested.

\subsubsection{Test Apparatus and Procedure}

The tests were carried out on the Sodium Loop Safety Facility (SLSF) at the Engineering Test Reactor under the direction of the Hanford Engineering Development Laboratory.

Each LOPI was initiated from steady state full power full-flow conditions. Over the first 0.5 seconds of the transient the inlet flow was dramatically reduced. At 0.65 seconds into the transient, the reactor was scrammed. The test section was returned to full flow after approximately 3 seconds from time zero.

The Boiling Window Tests were initiated at a steady-state flow of $1.95 \mathrm{~kg} / \mathrm{sec}$. The flow was linearly reduced to its "low flow" value in 0.5 seconds, where it was held for a specific time, and then linearly returned to its initial state in 0.5 seconds.

The geometric parameters of the fuel bundle and the characteristics of the tests are given in table 6.2 and 6.3 respectively. Figure 6.6 shows the axial cell locations used in the NATOF-2D simulations.

\subsubsection{Tests Chosen For Simulation}

From the available results, a total of four test were 
Table 6.2

Geometric Parameters of the W-1 SLSF Bundle

Number of Pins

Pin Diameter (m)

Fuel Pellet Diameter (m)

Wire Wrap Diameter ( $m$ )

inner pins

outer pins

Fuel Pitch $(m)$

Pitch/Diameter Ratio

Flat-to-Flat (m)

Length of Active Fuel

Axial Power Distribution, Cosine

$$
\text { peak/mean }
$$

Radial Power Distribution, Cosine

$$
\text { peak/mean }
$$

1.0
19

$5.842 \times 10^{-3}$

$4.94 \times 10^{-3}$

$1.422 \times 10^{-3}$

$7.11 \times 10^{-4}$

$7.264 \times 10^{-3}$

1.25

$3.26 \times 10^{-2}$

0.9144

Fuel

Uranium-Plutonium mixed oxide, Pu 25\% of total mass

Fill Gas 10\% Helium-Neon

Inlet Liquid Temperature $\left({ }^{\circ} \mathrm{C}\right)$ 388 . 
Table 6.3

Power and Flow Rates of the W-1 SLSF Tests

LOPI $2 \mathrm{~A}$

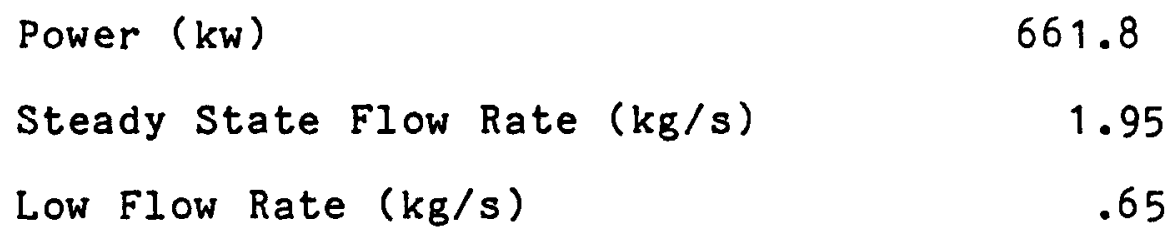

LOPI 4

Power (kw)

705.3

Steady State Flow Rate $(\mathrm{kg} / \mathrm{s}) \quad 1.95$

Low Flow Rate $(\mathrm{kg} / \mathrm{s}) \quad .65$

BWT 2:
Power (kw)
348.3
Steady State Flow Rate $(\mathrm{kg} / \mathrm{s})$
1.95
Low Flow Rate ( $\mathrm{kg} / \mathrm{s})$
.47

BWT $7 \mathrm{~B}^{\prime}$
Power (kw)
661.8
Steady State Flow Rate $(\mathrm{kg} / \mathrm{s})$
1.98
Low Flow Rate $(\mathrm{kg} / \mathrm{s})$ 
Height (m)

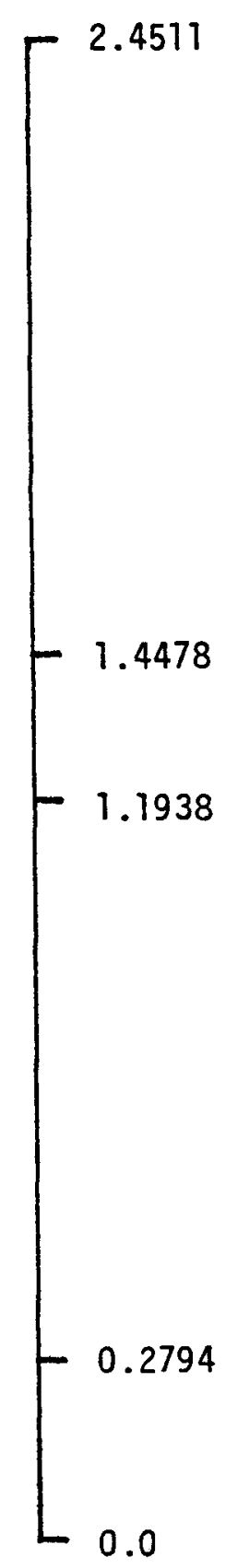

Axial

Cell Leve1

39

29

24

6

Figure 6.6 Location of Cells used in the NATOF-2D Simulation of W-1 SLSF Experiment 
chosen for simulation with NATOF-2D. Two tests were selected from the LOPI tests, and two from the BWT tests. These tests were selectively chosen to provide a full range of transients: from single phase calculation to a full dryout simulation.

The first LOPI simulation is LOPI $2 \mathrm{~A}$. In this test, the maximum coolant temperature reached $941^{\circ} \mathrm{C}$ and showed no indication of boiling. This test will provide some calibration information for the future test of the series.

The second LOPI simulation is LOPI 4. The maximum coolant temperature reached was $956^{\circ} \mathrm{C}$, and the data showed about 0.5 seconds of boiling. Failure of one of the thermocouples used for the test section power calculations resulted in LOPI 4 being run at approximately 5\% overpower $(15.12 \mathrm{kw} / \mathrm{pin})$. This transient will test the sensitivity of the code to such an occurrence.

The first BWT simulation is BWT 2\%. In this test approximately 0.8 seconds of bolling was observed. The coolant temperature reached $953^{\circ} \mathrm{C}$ at a pin power of 7.5 $\mathrm{kw} / \mathrm{ft}$. The test section was held at $24 \%$ of full flow for a duration of 4 seconds. This test offers the opportunity of simulating a low-power/low-flow sodium boiling transient.

The second BWT to be simulated is BWT7B'. This is the most interesting and demanding test to be run by the code. Approximately 2.0 seconds of boiling occurred before clad dryout at a pin power of $14.4 \mathrm{kw} / \mathrm{ft}$. The test section had 
$38 \%$ of full flow for a period of 3.0 seconds. Inlet flow oscillations, flow reversal and dryout is the worst case hypothesized for sodium boiling codes. Ability to model this sequence of events will severly test the limitations of NATOF-2D.

The input files used in the NATOF-2D simulation of the W-1 SLSF Tests are given in Appendix $C$. 


\subsection{W-1 SLSF Simulation Results \\ 6.4.1 LOPI $2 \mathrm{~A}$}

The LOPI $2 \mathrm{~A}$ simulation was carried out under velocity/pressure boundary conditions in order to accurately duplicate the experiment's inlet flow rate (shown in Figure 6.7). A comparison between the NATOF-2D predicted liquid temperature and the experiment's result is shown in Figure 6.8. The shape of the two curves are fairly close, with NATOF-2D predicting slightly higher temperatures. Differences are to be expected however, since average cell temperatures are being compared with temperatures taken at a point. This simulation shows that the fuel pin properties used in the code are quite good, and accurately model the response of real fuel pins during a reactor scram.

Figure 6.9 compares the temperatures at the end of the heated zone for the central and edge channels. This figure shows how the edge channel takes longer to respond to the transient due to the effects of heat losses to the can.

\section{4 .2 LOPI 4}

The LOPI 4 simulation was also run under velocity/pressure boundary conditions. The flow rate is shown in 6.10 . As in the experiment, there was approximately 0.5 seconds of boiling. The NATOF-2D predicted temperature at the end of the heated zone closely matched the experiment for the central channel (Figure 


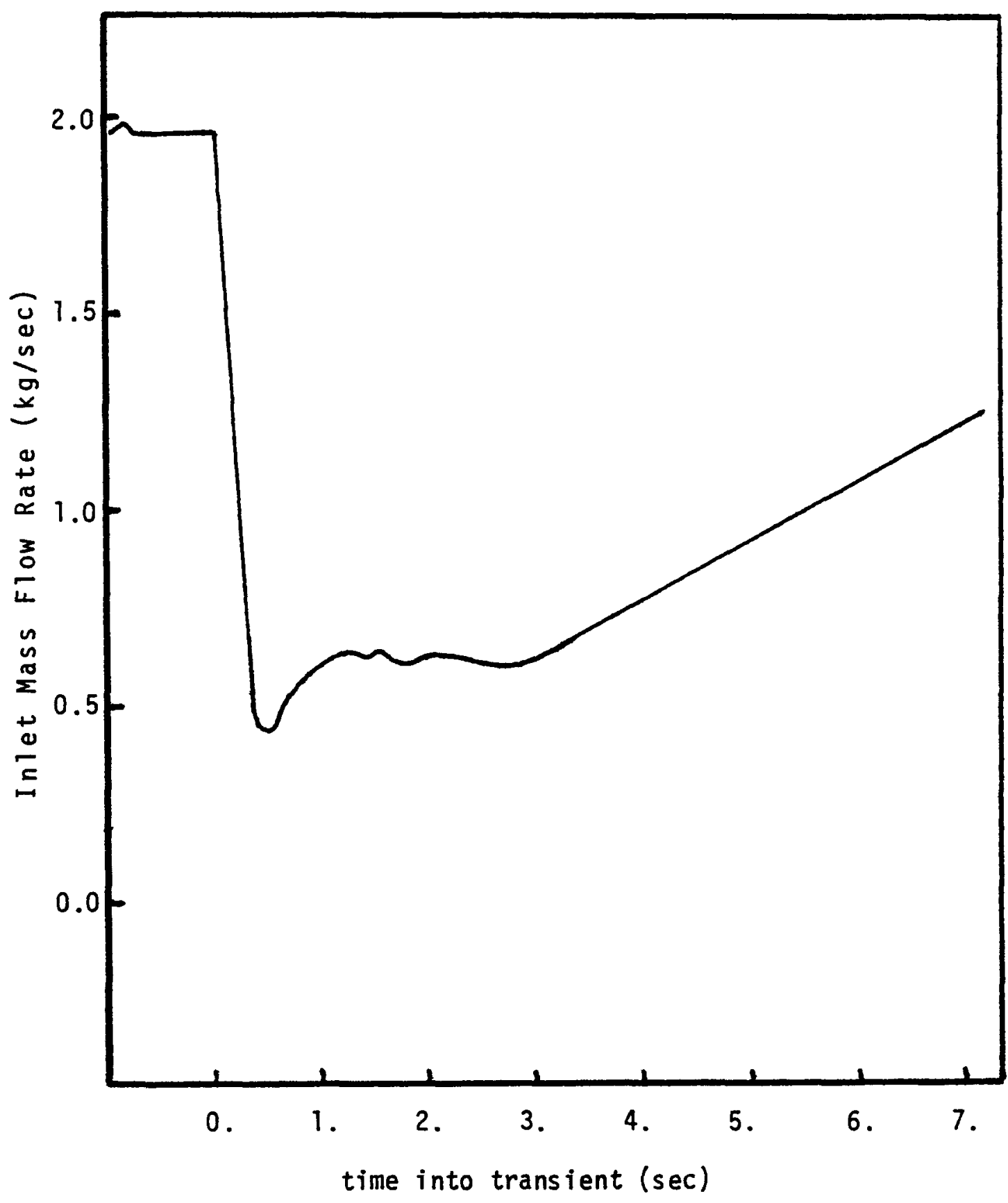

Figure $6.7 \quad W-1$ SLSF Test LOPI $2 A$ Experiment Inlet Mass Flow Rate 


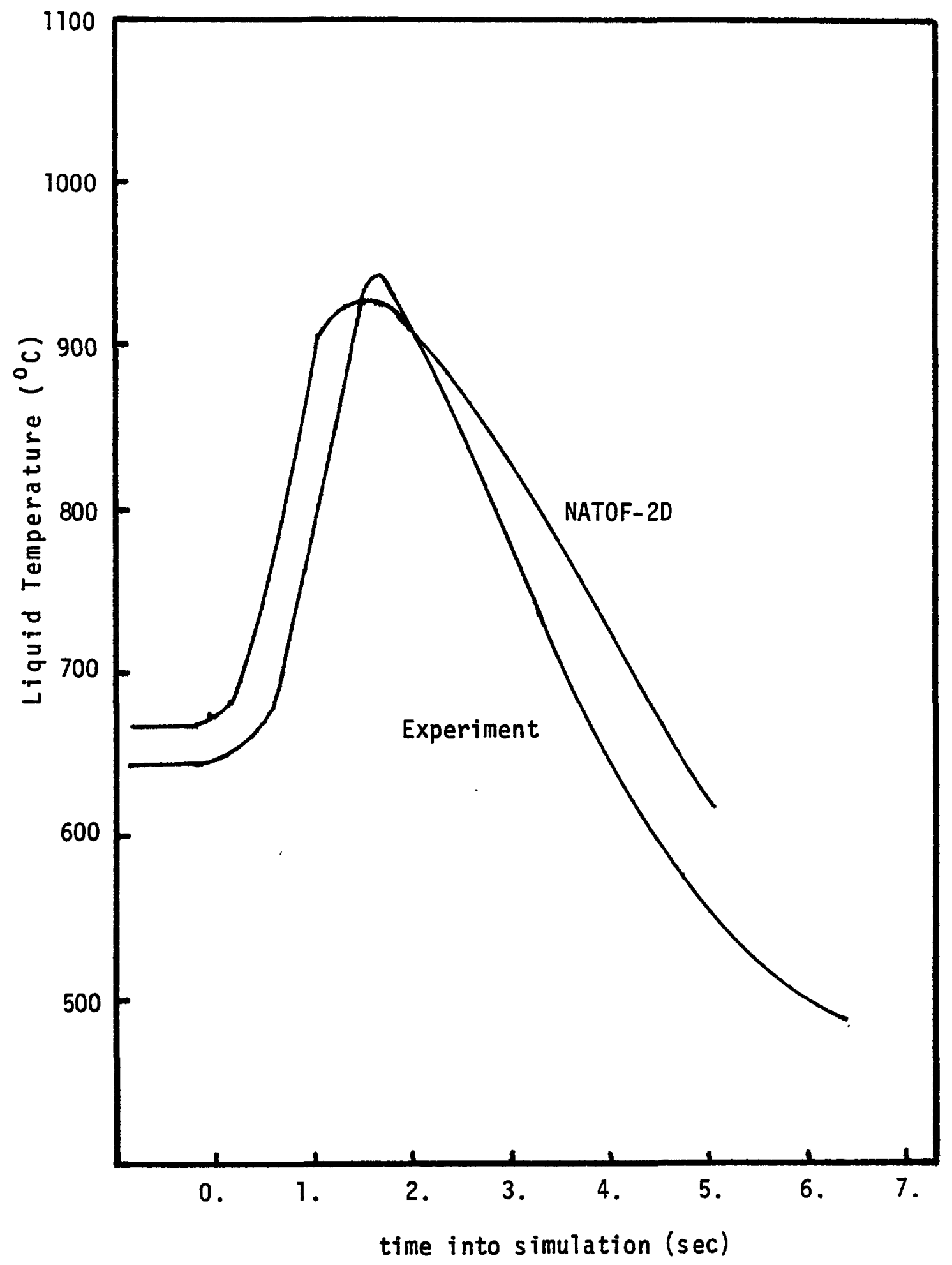

Figure 6.8 A Comparison Between Experiment and NATOF-2D Predicted Temperature Histories at the End of the Heated Zone for the Central Channel ( $W-1$ SLSF TeSt LOPI 2A) 


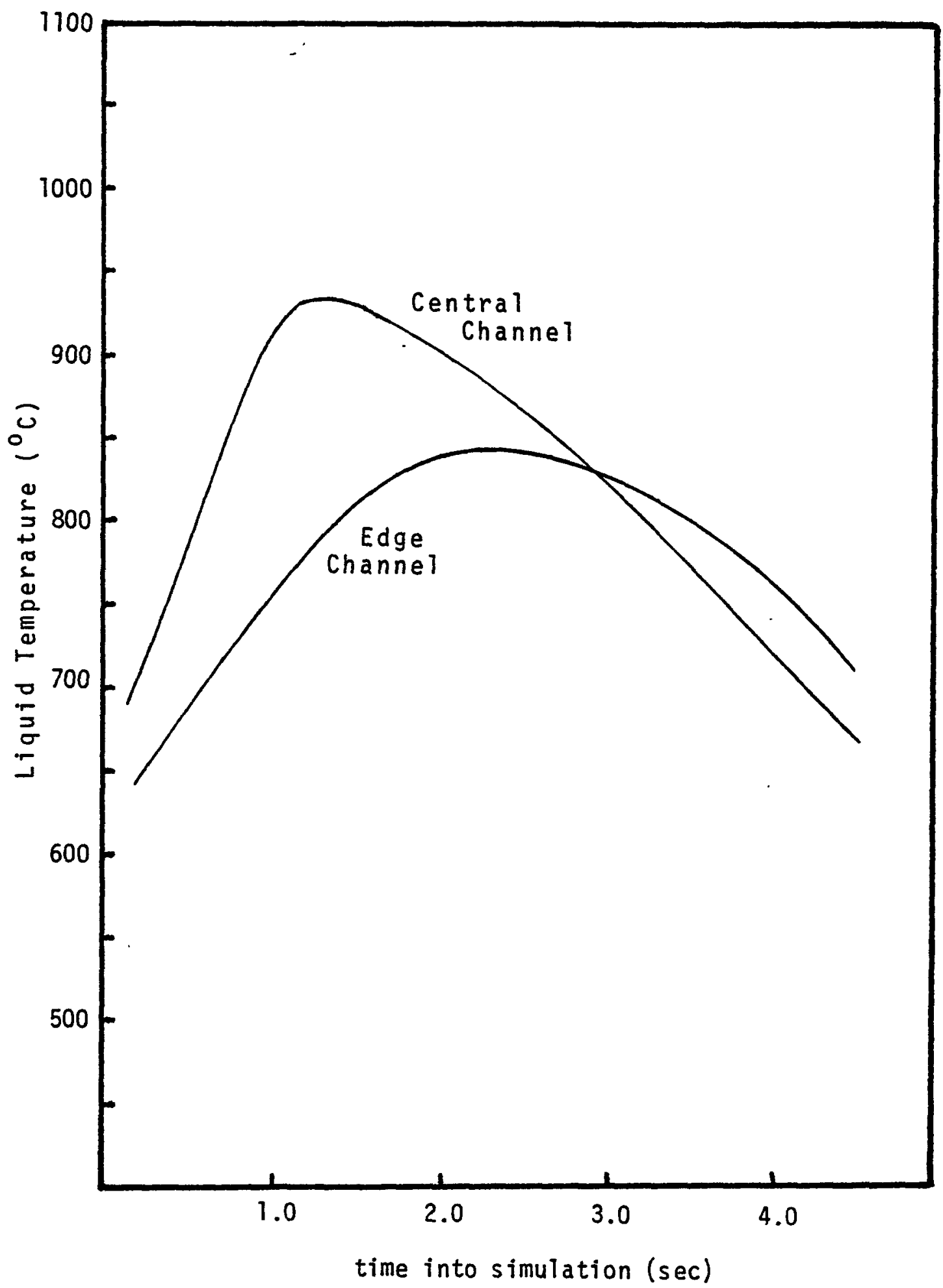

Figure 6.9 A Comparison of NATOF-2D Central and Edge Channel Temperature Histories at the End of the Heated Zone ( $W-1$ SLSF Test LOPI 2A) 


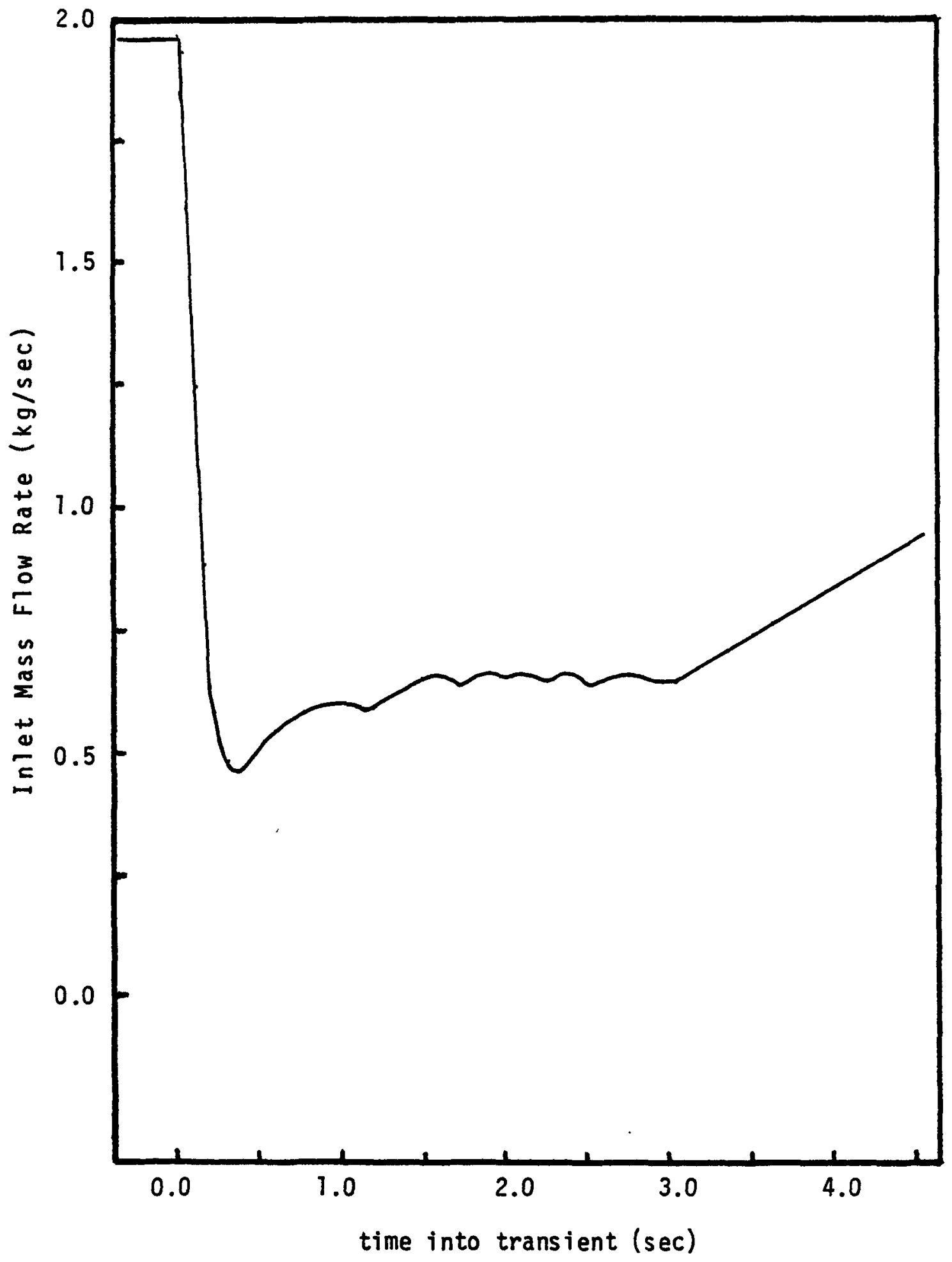

Figure 6.10 W-1 SLSF Test LOPI 4 Experiment Inlet Mass Flow Rate 
6.11), but not as well for the edge channel (Figure 6.12). Void maps for the central and middle channels are shown in Figure 6.13. Although these maps are for voids of 0.1 , they nearly overlap the vold maps for 0.9. A negligible void was found in the edge channel. In and near the voided regions the radial velocities were large, as the coolant traveled.from the center of the bundle to the edge. This demonstrates the value of a two-dimensional model in simulating boiling transients.

\section{4 .3 BWT 2 '}

The BWT 2' simulation was a low power-low flow transient. The test section inlet mass flow rate is shown in Figure 6.14. The temperature histories at the midplane and end of the heated zone for the central channel are shown in Figures 6.15 and 6.16. Unlike the experiment, which had 0.8 seconds of boiling, no sodium boiling occurred in the simulation. The liquid reached a maximum temperature of $931^{\circ} \mathrm{C}$, approximately $20^{\circ} \mathrm{C}$ below saturation.

The exact reason for this result is not known, but possibly may be associated to overestimating the heat capacity of the fuel rods. The reported inlet mass flow rate also could have been too high. In a low power transient such as this, the necessity of using cell averaged temperatures, results in lower peak temperatures. Even 


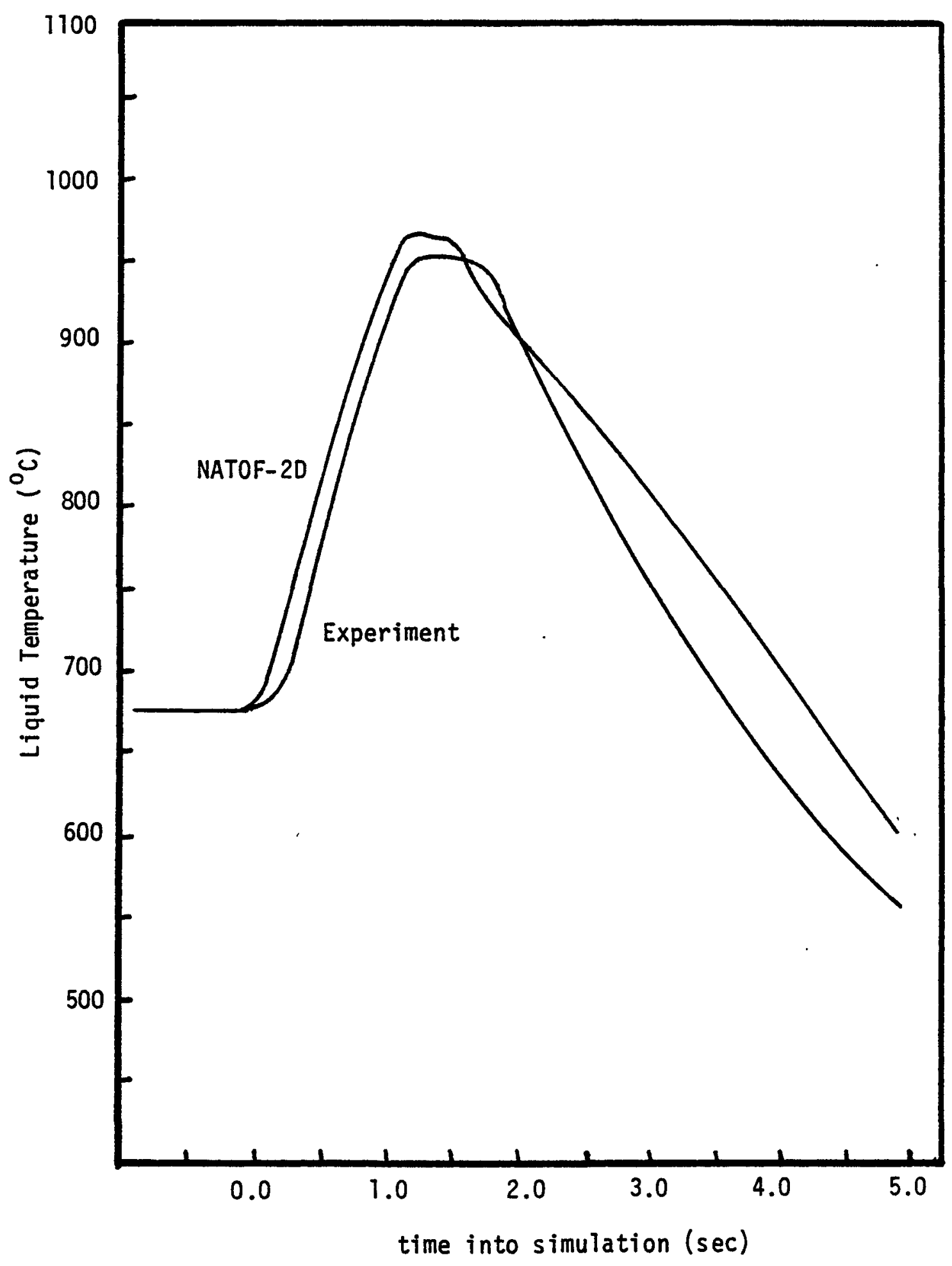

Figure 6.11 A Comparison Between W-1 SLSF Test LOPI 4 and NATOF-2D Temperature Histories at the End of the Heated Zone for the Central Channel 


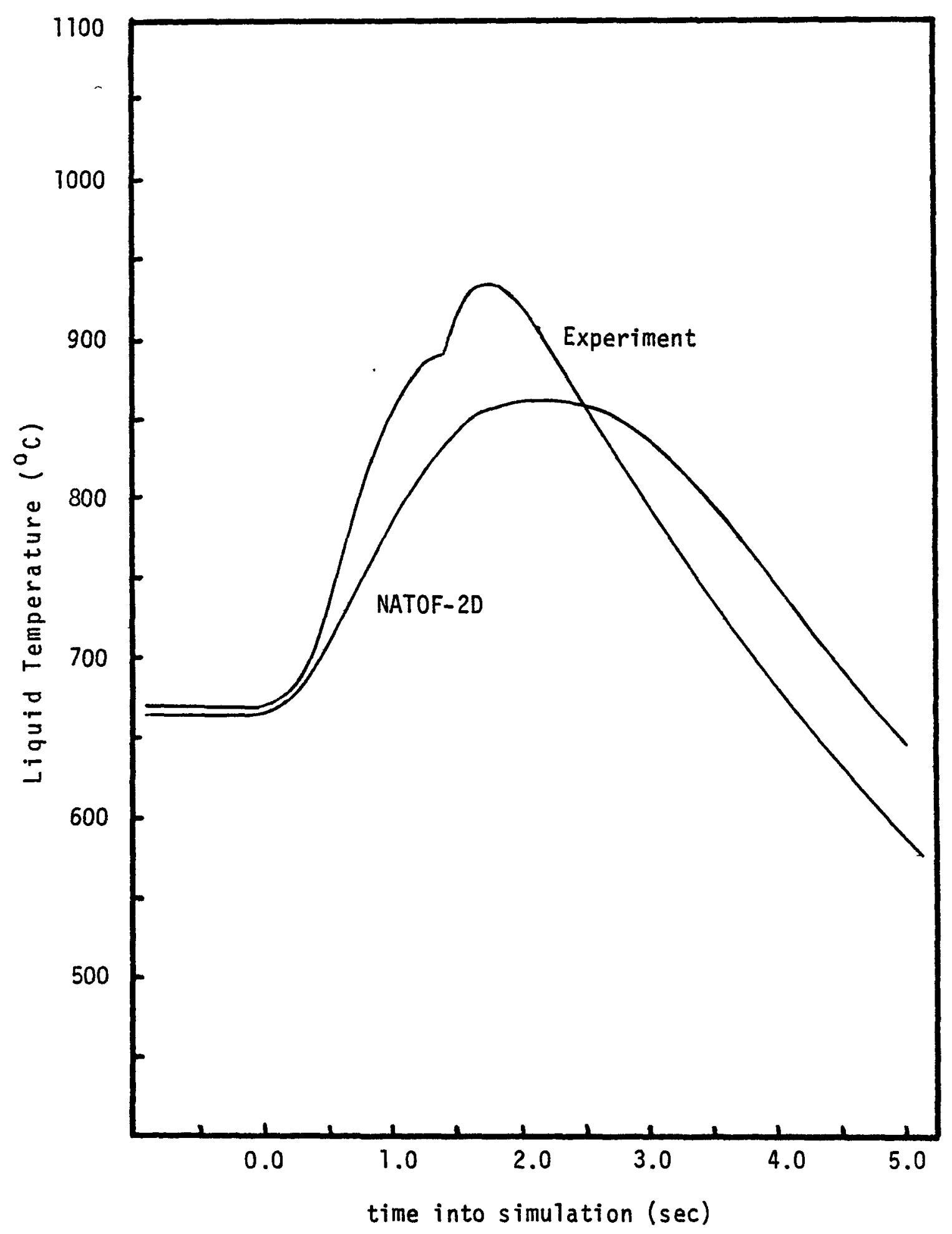

Figure 6.12 A Comparison Between W-1 SLSF Test LOPI 4 and NATOF-2D Temperature Histories at the End of the Heated Zone for the Midplane Channel 


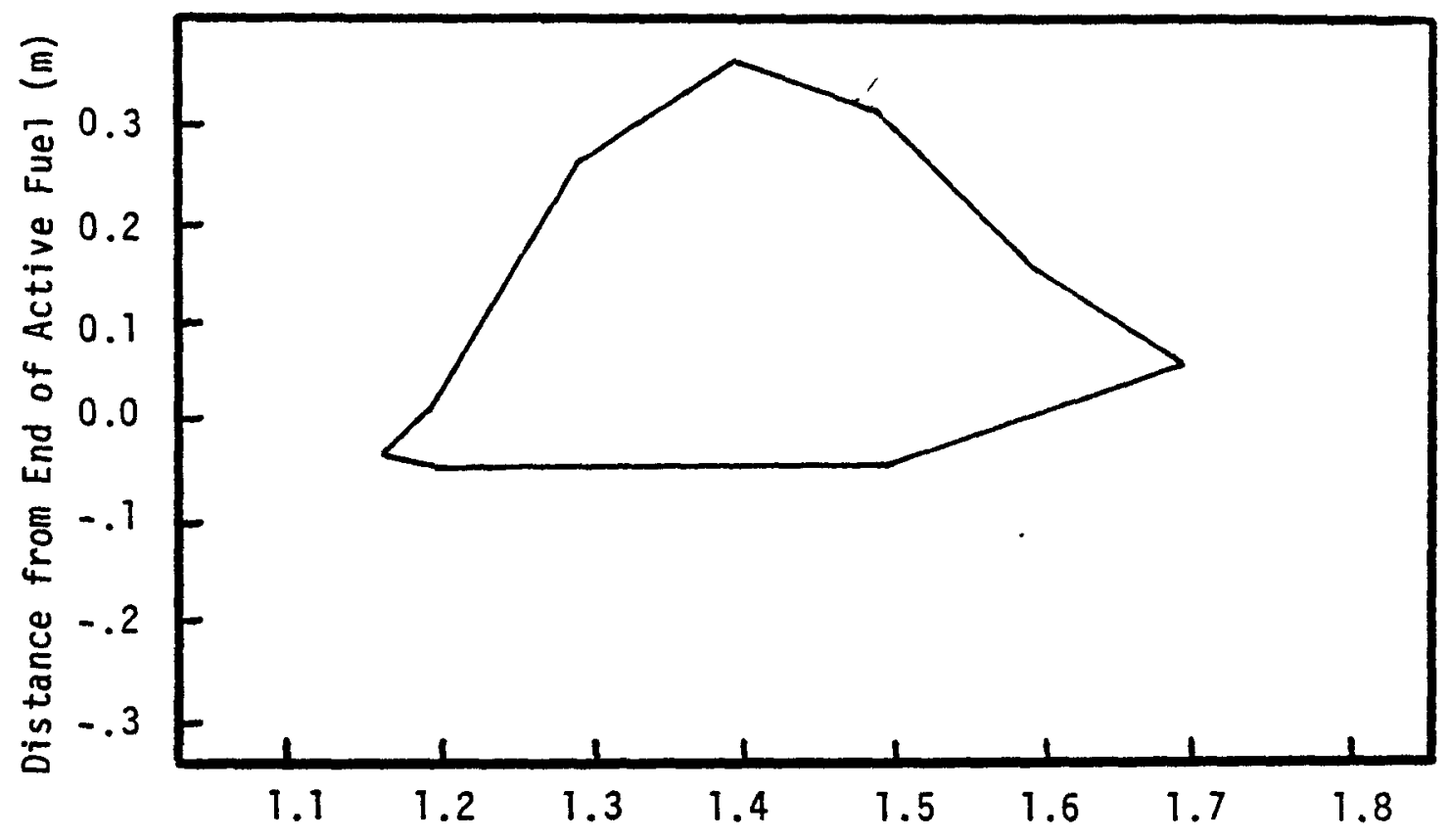

time into simulation (sec) - Central Channel

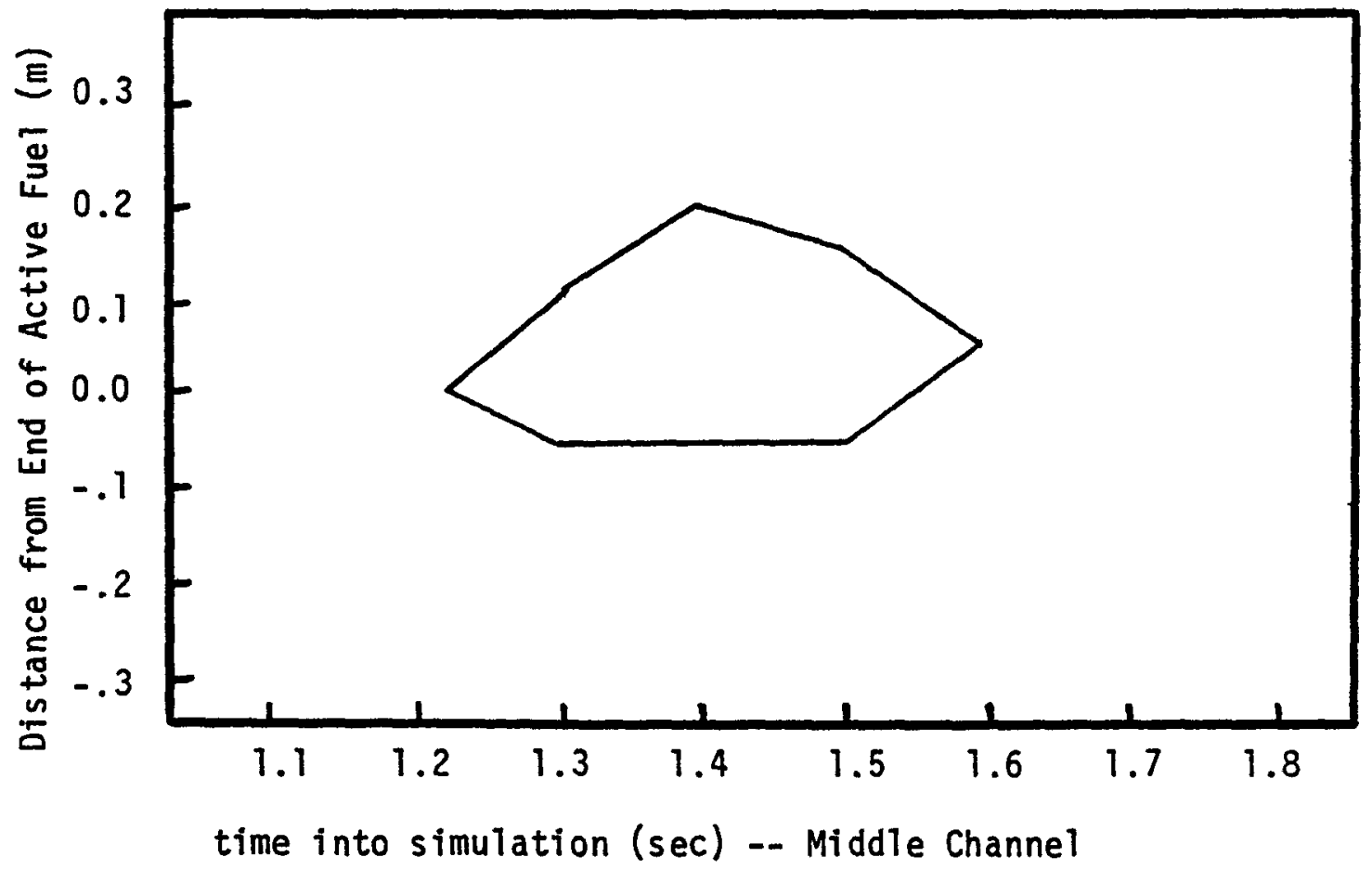

Figure 6.13 NATOF-2D Void Maps for $W-1$ SLSF Test LOPI 4 for the Central and Middle Channels Void $=0.1$ 


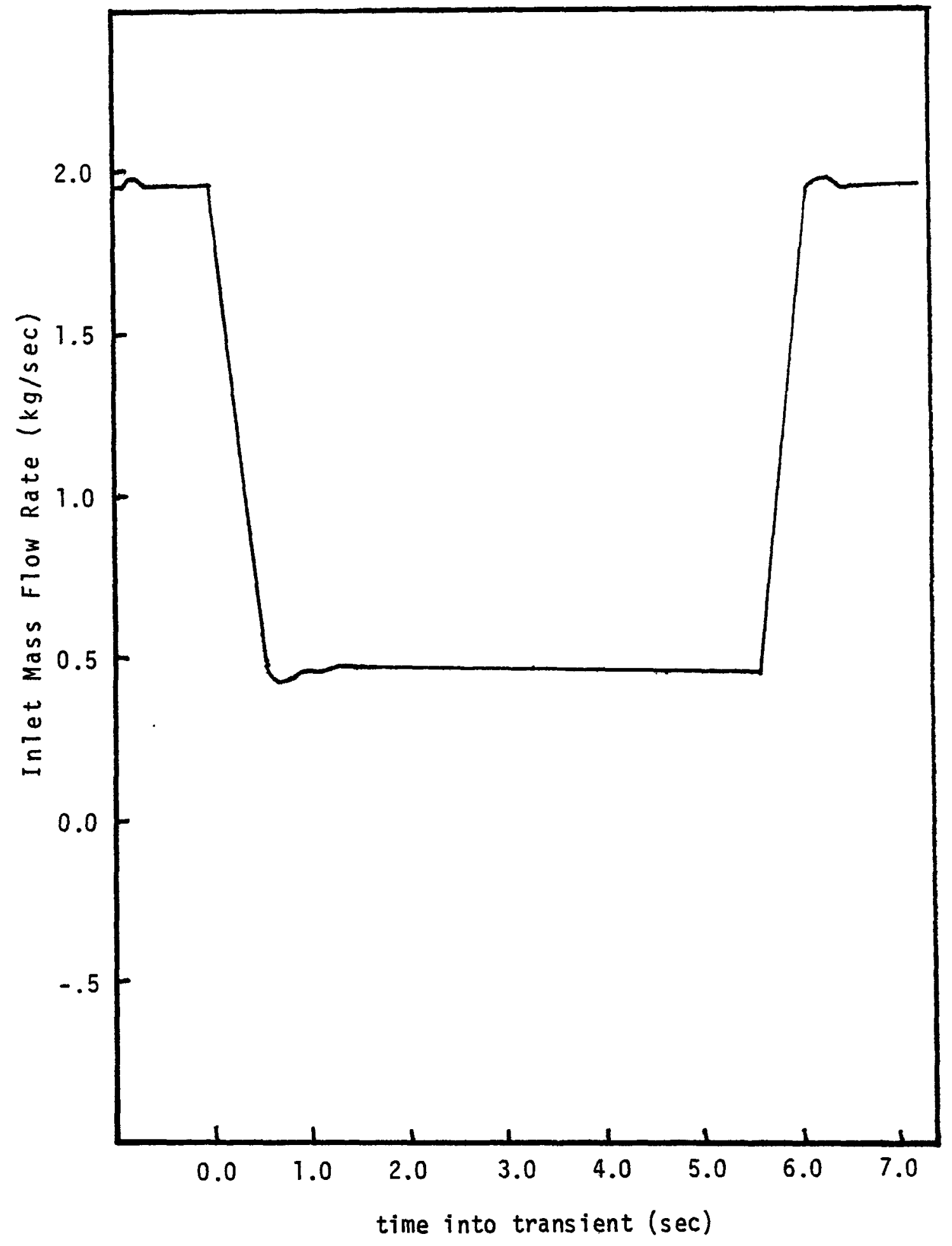

Figure 6.14 W-1 SLSF Test BWT 2' Inlet Mass Flow Rate 


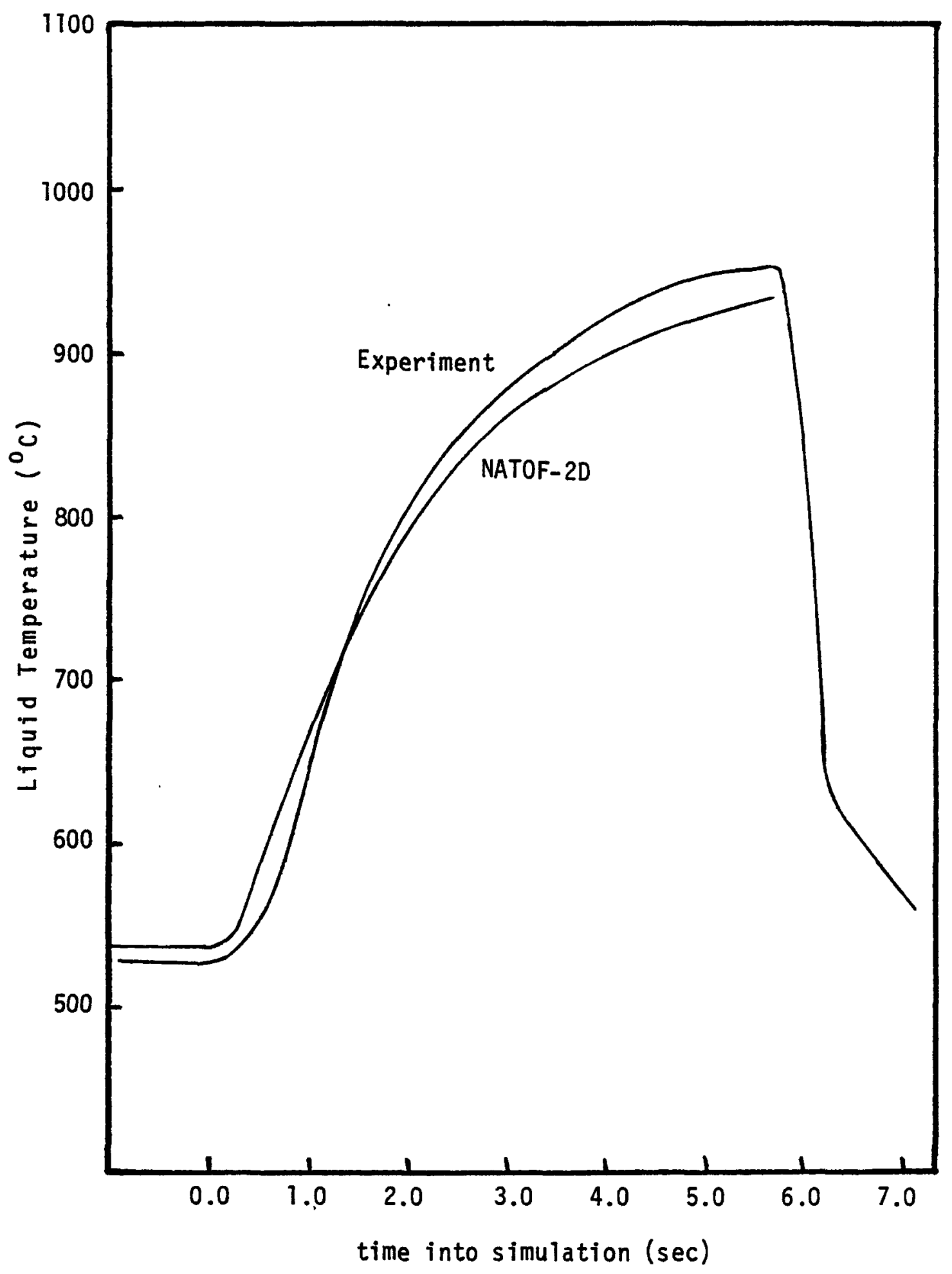

Figure 6.15 A Comparison Between W-1 SLSF Test BWT 21 and NATOF-2D Temperature Histories at the End of the Heated Zone for the Central Channel 


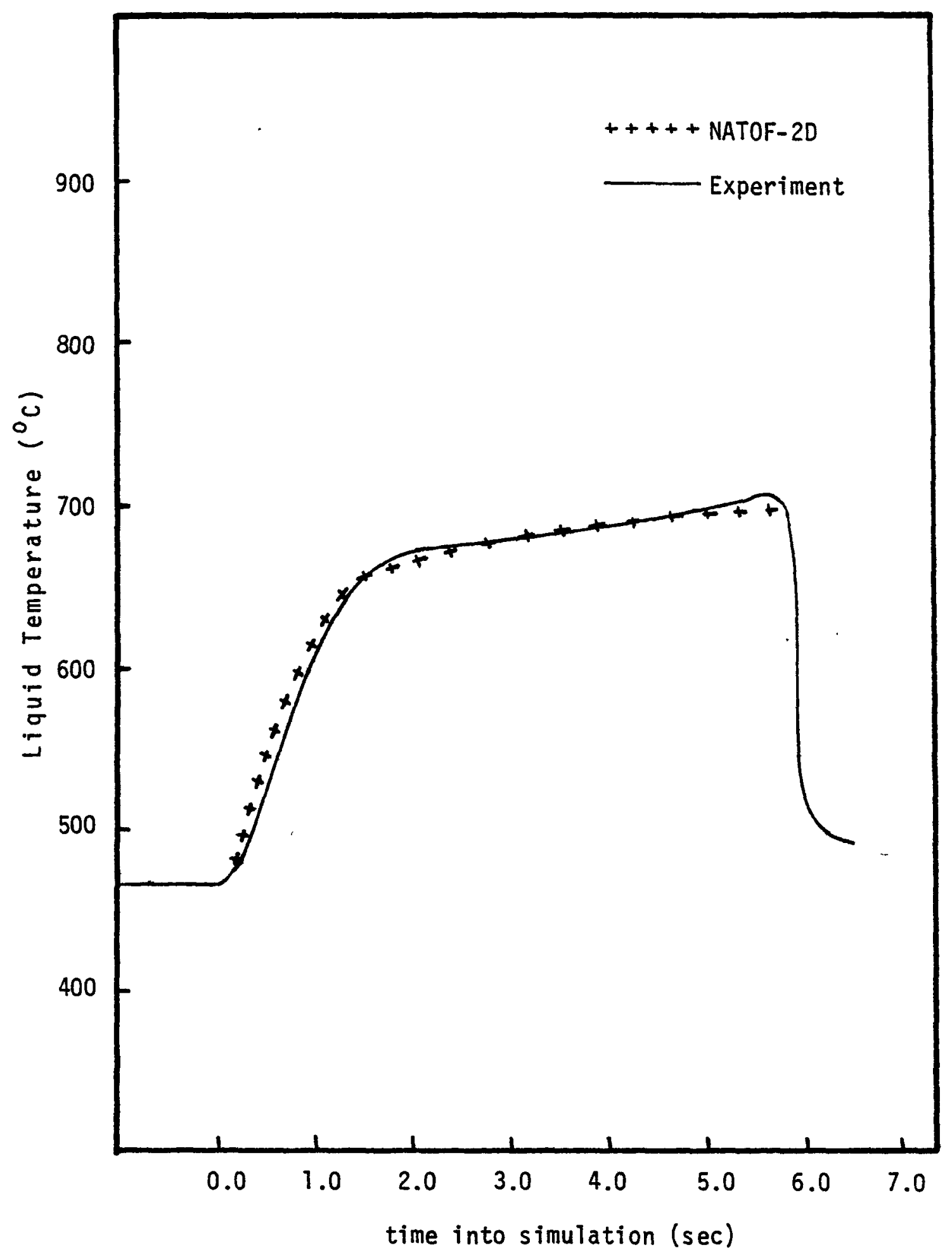

Figure 6.16 A Comparison Between W-1 SLSF Test BWT 2' and NATOF-2D Temperature Histories at the end of the Heated Zone for the Central Channel 
using cells $5 \mathrm{~cm}$ in length resulted in axial cell to cell temperature differences of $12^{\circ} \mathrm{C}$.

Further simulations of this experiment have revealed that this result is not caused by overestimating the radial fluid-to-fluid heat transfer or the hexcan heat capacity.

\subsubsection{BWT $7 \mathrm{~B}^{\prime}$}

BWT $7 \mathrm{~B}^{\prime}$ was a high power boiling window transient. The simulation was carried out under velocity/pressure boundary conditions until the point of boiling inception, when pressure/pressure boundary conditions were used. When the code reached the dryout point, at which time the switch is made in interfacial mass and energy exchange correlations, the code reduced to extremely small timesteps $\left(10^{-7} \mathrm{sec}\right)$ which were below an acceptable level.

The reason for this can again be traced to the problems of boiling and condensation. Even at high void fractions, the liquid phase is often subcooled to a large extent. Thus the switch in correlations requires drastic changes in temperature. The vapor phase must act as the source of this heat, and since it obeys the perfect gas law, large pressure changes are necessary.

In order to obtain results, the convergence criteria was relaxed $\left(\delta P=10^{-2}\right)$. It was found that with this large convergence criteria it was possible to set $C F=1.0$ and run 
for 0.7 seconds during boiling, until small timesteps were required. The simulation offers some interesting observations when compared to the same case which was run with $C F=0.01$.

For $C F=0.01$, flow reversal occurred at 0.25 seconds after boiling inception, while for $C F=1.0$, flow reversal didn't occur until 0.45 seconds. Figure 7.17 compares the experiment's flow rate to that predicted by NATOF-2D (for $C F=1.0)$. A comparison of void maps is given in figures 6.18 and 6.19 for the central and edge channels. As can be seen the large condensation rate keeps the void centralized. Figure 6.20 compares the temperatures at the end of the heated zone for the central channel. 


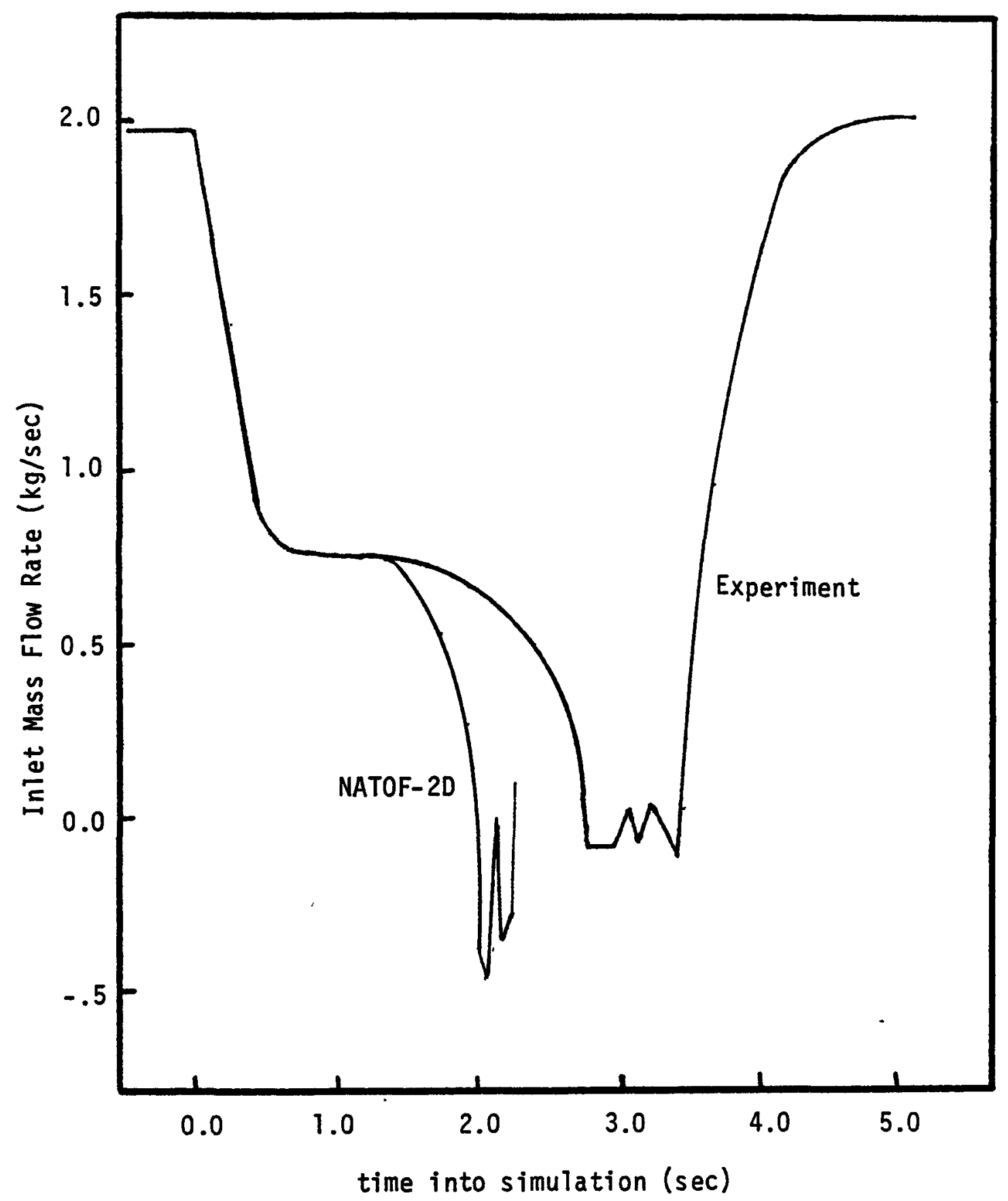

Figure 6.17 A Comparison Between W-1 SLSF BWT 7B' and NATOF-2D Predicted Inlet Mass Flow Rates 


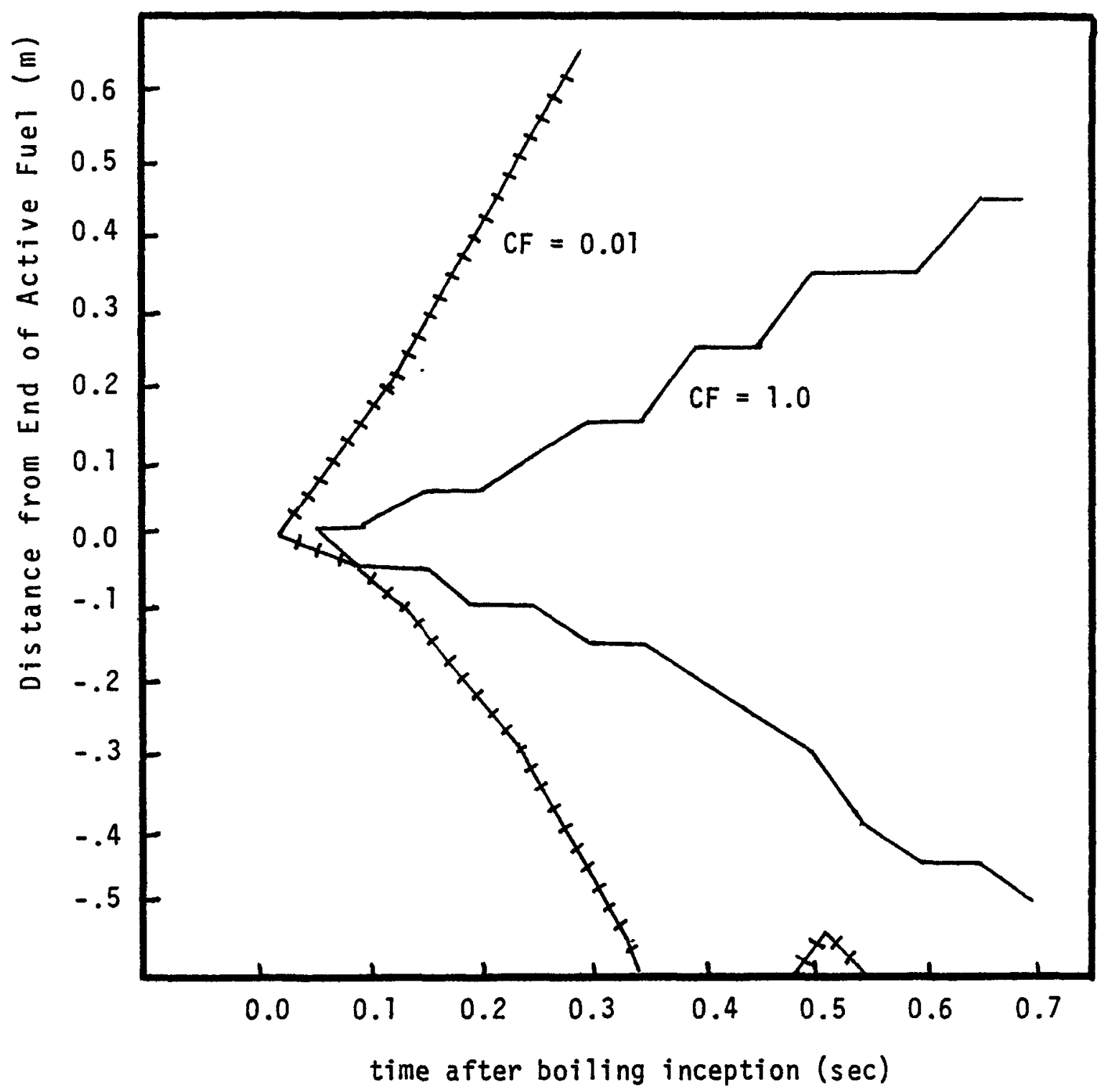

Figure 6.18 A Comparison of NATOF-2D Central Channel Void Maps for $C F=1.0$ and $C F=0.01-$ Void $=0.9$ (W-1 SLSF Test BWT 7B') 


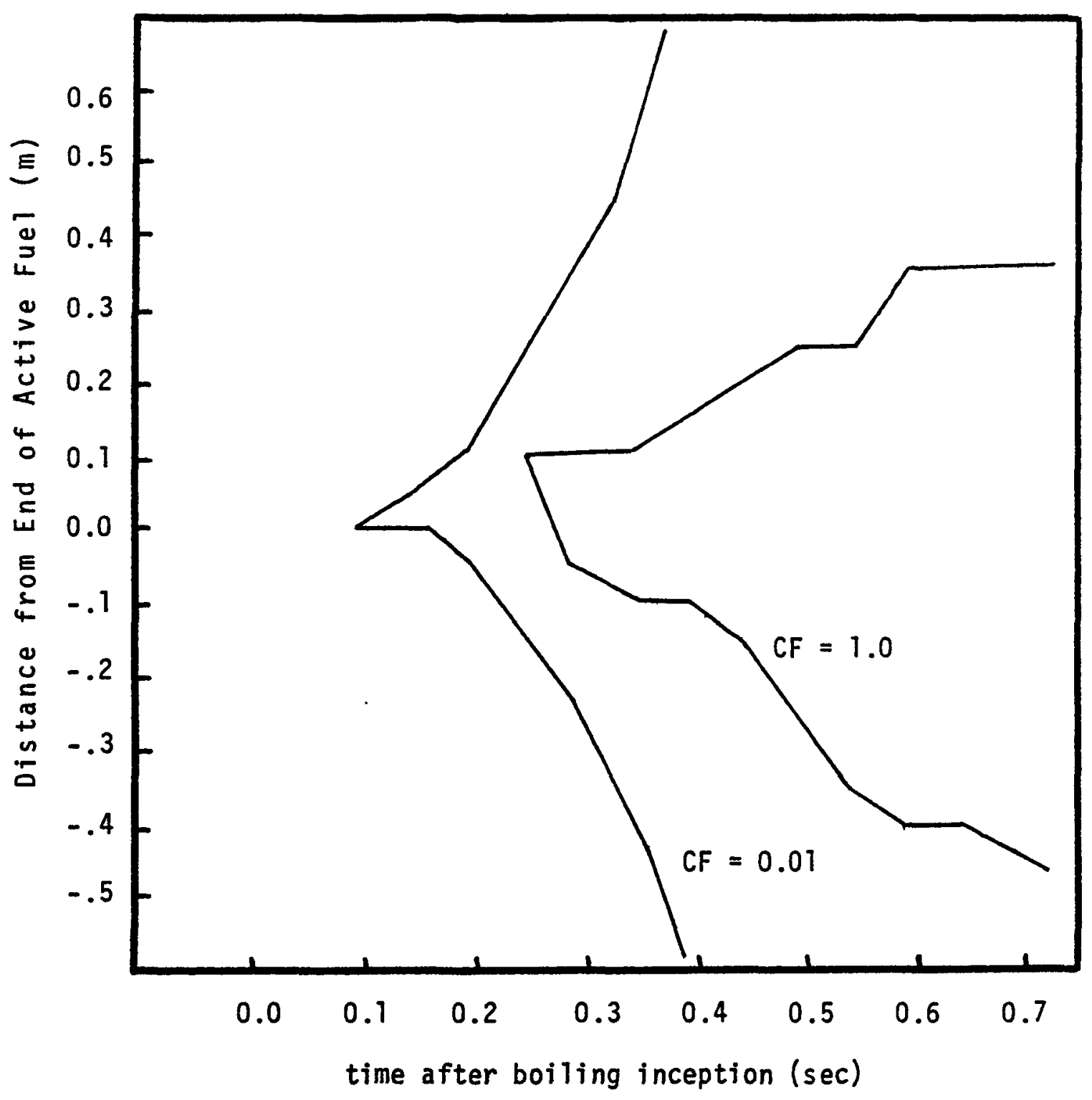

Figure 6.19 A Comparison of NATOF-2D Edge Channel Void Maps for $C F=1.0$ and $C F=0.01-$ Void $=0.9$ (W-1 SLSF Test BWT 7B') 


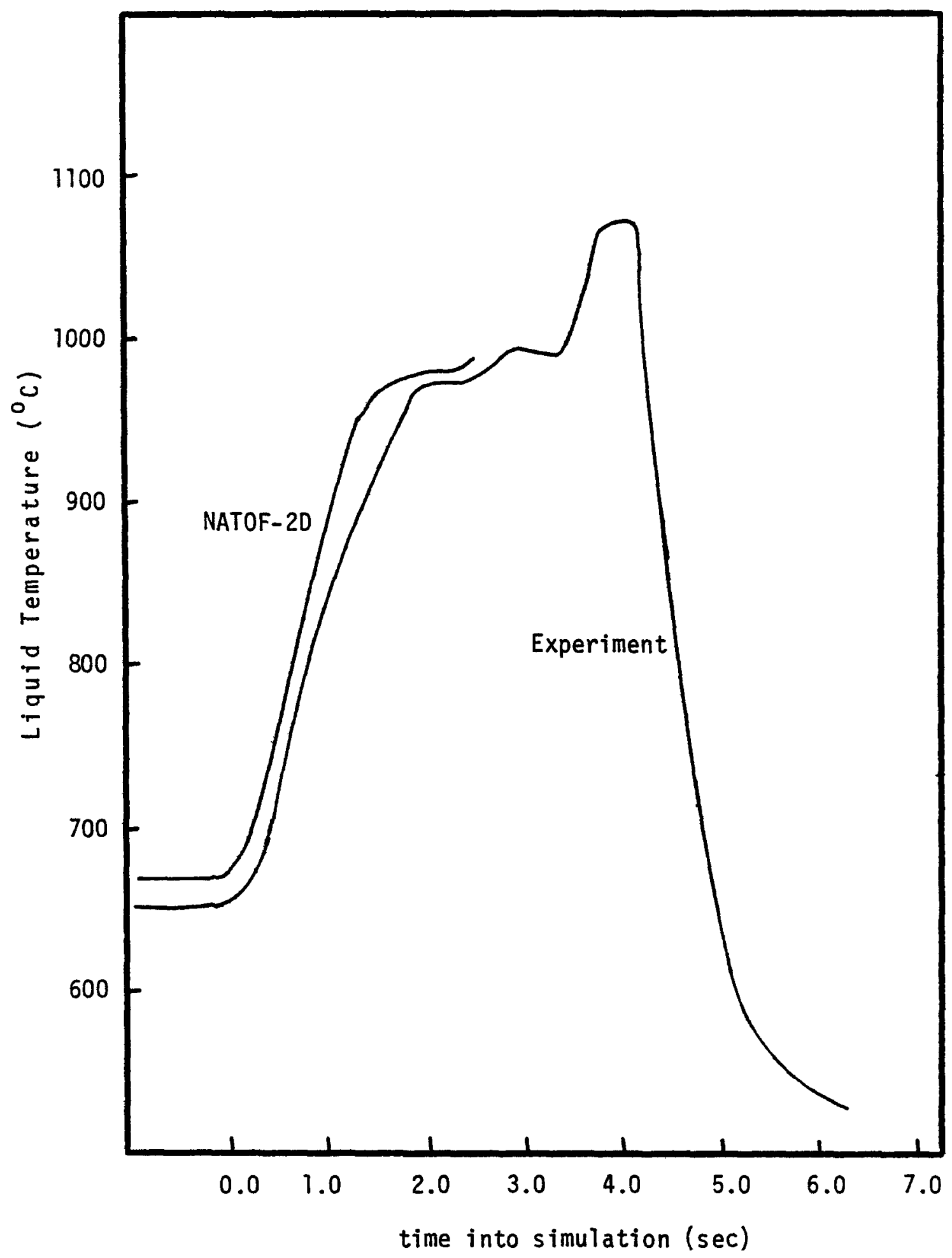

Figure 6.20 A Comparison Between NATOF-2D and W-1 SLSF BWT 7B' Temperature Histories at the End of Heated Zone for the Central Channel 


\section{Chapter 7}

CONCLUSIONS AND RECOMMENDATIONS

\subsection{Conclusions}

The models implemented in NATOF-2D performed well during the simulations described in Chapter 6 . In particular, the fluid heat conduction model gave a much improved temperature distribution comparable to the gradients found in the experiments. The direct solution technique allowed the simulations to be performed in a more detailed fashion, and within the limits imposed by computational costs.

Little more can be said about the interfacial momentum and energy exchange rates than what is described in Chapter 2. The effects of these terms are difficult to correlate with experimental results, since two-phase flow interactions are almost never directly measured. However. the sodium boiling simulations showed that the models gave physically reasonable results.

Problems were encountered during boiling in the test simulations, and most of these were covered in Chapter 5. For this reason, it is difficult to judge the effect of the mass exchange model of the code. However, it was possible to achieve full condensation with the model for a relaxed convergence criteria. 
The smaller the axial mesh cell spacing in the simulation, the more confined is the voided areas of the core, and better condensation is achieved. Since the smaller volume of the cell leads to reduced pressure drop changes upon boiling, increased convergence could be attained. 
7.2 Recommendations

NATOF-2D, in its present form. is an adequete model for single phase calculations. However, before extensive use of the code can occur, it will be necessary to overcome the difficulties of boiling and condensation. It is a requirement to have the evaporation and condensation rates be well behaved, since they effect the void fraction, flow reversal and heat transfer from the fuel pins. Once this has occurred, NATOF-2D can be a valuable tool in LMFBR accident analysis.

Throughout the present effort, there has persisted the problem of modeling the transition between the vapor and liquid phases without resorting to very small timesteps. Initially, the focus of this work had been the development of constituative relations in the belief that this problem could be overcome. However, continued work has shown that it is not the properties of the constituative relations which cause the difficulties, but rather the properties of the transients being simulated. The numerical scheme utilized by NATOF-2D, particularly the Newton Iteration method, is extremely powerful for well behaved functions. However, the properties of sodium and LMFBRs have severely tested the limits of the method, and have posed a choice: either small timesteps must be taken to achieve full condensation, or else the relaxed convergence criteria associated with larger timesteps must be accepted. 
The results of this work demonstrate the need for improving the capability of the iteration scheme. Rather than the simple reduction of timestep sizes, it would be cost effective to develop a method which is able to solve for the unknowns of the problem at each timestep, even if more iterations than before are required. of particular value in this line of development would be to use the semi-implicit nature of the calculation to cause the derivatives of the problem to be well behaved.

The NATOF-2D simulation of THORS Bundle $6 \mathrm{~A}$ Test $71 \mathrm{H}$, Run 101 demonstrated the need for improving the model for heat losses to the hexcan, and for including heat losses to the environment.

Another area for future work would be the development of a mechanism for assuming a temperature gradient in the cell. replacing the assumed flat profile presently used in the code. This would be necessary to achieve greater detail in the simulation results since further decreases in the axial mesh spacing beyond those used in the simulations are not possible due to computational constraints. An assumed gradient would allow boiling inception to occur in a more localized manner, reducing the effects of full cell boiling. 
References

1. Granziera, M.R., and Kazimi, M.S., "A Two Dimensional, Two Fluid Model for Sodium Boiling in LMFBR Assemblies," MIT Energy Laboratory Report MIT-EL-80-011, May 1980

2. Engel, F.C., Markley, R.A., and Minushkin, B. "The Effect of Heat Input Patterns on Temperature Distributions in LMFBR Blanket Assemblies," Westinghouse Electric Corp. Advanced Reactor Division, Madison, Pennsylvania (1978)

3. Yeung.M.R., "A Multicell Slug Flow Heat Transfer Analysis for Finite LMFBR Bundles," PhD. Thesis, Department of Nuclear Engineering, MIT, (1978)

4. Ribando, R.J., et al.,"Sodium boiling in a Full-Length 19-Pin Simulated Fuel Assembly (THORS Bundle 6A)", ORNL/TM-6553 (1979)

5. Rothrock, R.B. and Henderson, J.M., "Description and Results of the W-1 SLSF Experiment Fuel Pin Heat Release Characteristics and Sodium Boiling Stability", Hanford Engineering Development Laboratory, Energy Contract No. EY-76-C-14-2170

6. Schrage, R.W., A Theoretical Study of Interphase Mass Transfer, Columbia University Press, N.Y. (1953)

7. Collier, J.G., Convective Boiling and Condensation, McGraw-Hill, United Kingdom, 1972

8. Wilson, G., and Kazimi, M.S., "Development of Models for the Sodium Version of the Two Phase, Three Dimensional Thermal Hydraulics Code THERMIT," MIT-EL-80-010

9. Knight,D.D., "SLSF W-1 LOPI Experiments Preliminary Evaluation of Data," ST-TN-80015, October 1979

10. Martin,R.S., and Wilkinson, J.H., "Solution of Symmetric and Unsymmetric Band Equations and the Calculation of Eigenvectors of Band Matrices," Numerische Mathematic 9(4) 1967

11. Franklin, Joel N., Matrix Theory, Prentice Hall. Inc., Englewood Ciiffs, N.J. (1968) 
12. Bell, W.W., Matrices for Scientist and Engineers, Van Nostrand Reinhold Co., New York, N.Y. (1975)

13. Nero, Anthony V., A Guidebook to Nuclear Reactors, University of California Press, Berkley and Los Angeles, California (1979)

14. Weast, Robert C. et al., CRC Handbook of Chemistry and Physics, The Chemical Rubber Company, Cleveland, Ohio (1969) 
Appendix A

SPECIFIED INLET VELOCITY AND MASS FLOW RATE

\section{A.1 Introduction}

In the simulation of sodium bolling transients, it is necessary to establish the proper flow rate through the bundle in order to obtain a temperature distribution which corresponds to the experimental results. Until now, this could only be accomplished by specifying the pressures at the inlet and at the outlet, and allowing the $\Delta P$ across the core to determine the mass flow rate. One difficulty associated with this method is that the pressures at the inlet and outlet are not always provided, and when provided, they often do not specify the effects of pressure drops due to valve throttling and fuel pin spacers. Another difficulty is that the flow rate is very sensitive to the $\Delta P$ across the core, and thus any small inaccuracies in the specified pressures can lead to large flow rate discrepencies. This has resulted in the use of a trial and error process to determine the necessary inlet and outlet pressures.

An alternative to this method would be to specify the velocity of the fluid or the total mass flow rate at the inlet, and infer the required inlet pressure from this. The advantage of these methods is that the proper flow rate 
could always be maintained.

This appendix will describe how the, relationship between pressures and fluid velocity is treated in NATOF-2D, and show how these equations can be modified in order that an inlet velocity or an inlet mass flow rate can be imposed. 
A.2 Treatment of the Momentum Equation

As described in the introduction to Chapter Four, the solution scheme employed by NATOF-2D relies on only the pressures relating the interactions between cells. This reduction to a pressure field solution is accomplished by treating the velocities at a cell boundary only as a function of the pressures in the two nelghboring cells in the momentum equation, and then substituting these relations into the mass and energy conservation equations. This section will describe the treatment of the momentum equations to obtain this result. Since the focus of this appendix is on the calculations of the velocity at the inlet, only the z-direction liquid and vapor momentum equations need be considered.

The time discretinized, finite difference form of the momentum equation for the vapor and liquid phases in the z-direction are:

vapor phase

$$
\begin{aligned}
& \left(\alpha \rho_{v}\right)_{1+\frac{1}{2} j}^{n}\left(\frac{\left(U_{v z}^{n+1}-U_{v z}^{n}\right)_{1+\frac{1}{2}} j}{\Delta t}+U_{v z i+\frac{1}{2} j} \frac{\left(\Delta z U_{v z}^{n}\right)_{1+\frac{1}{2}} j}{\Delta z}+\right.
\end{aligned}
$$

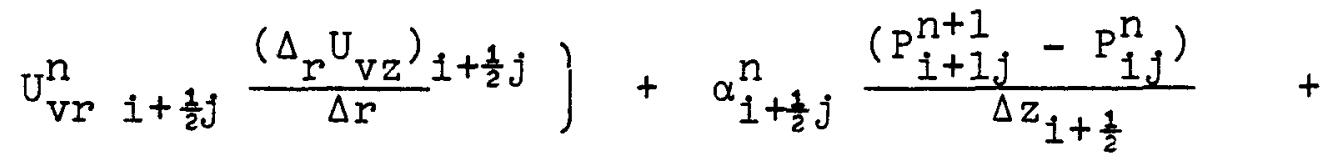

$$
\begin{aligned}
& \left(\alpha \rho_{v}\right)_{i+\frac{1}{2} j}^{n} g=-\left(M_{W z V}+M_{v \ell z}\right)_{i+\frac{1}{2} j}
\end{aligned}
$$




$$
\begin{aligned}
& \text { liquid phase } \\
& \left((I-\alpha) \rho_{\ell}\right)_{i+\frac{1}{2} j}^{n}\left(\frac{\left(U_{\ell z}^{n+1}-U_{\ell z}^{n}\right)}{\Delta t}{ }_{i+\frac{1}{2} j}+U_{\ell z i+\frac{1}{2} j}^{n} \frac{\left(\Delta_{z} U_{z}^{n}\right)}{\Delta z}{ }_{i+\frac{1}{2} j}\right. \\
& \left.+U_{\ell r i+\frac{1}{2} j} \frac{\left(\Delta_{r} U_{\ell z}\right)^{n}}{\Delta r} i+\frac{1}{2} j\right)+(I-\alpha)_{i+\frac{1}{2} j}^{n} \frac{\left(P_{1+1 j}^{n}-P_{i j}^{n}\right)}{\Delta z_{i+\frac{1}{2}}} \\
& +\left((I-\alpha) \rho_{\ell}\right)_{1+\frac{1}{2}}^{n} j^{\mathrm{g}}=-\left(M_{w z \ell}-M_{\ell v z}\right)
\end{aligned}
$$

where the interface momentum exchange terms are given by:

$$
\begin{aligned}
& M_{V \ell z}=(K+\eta \Gamma)^{n}\left(U_{v z}-U_{\ell z}\right)^{n+1} \\
& M_{\ell v z}=(K-(I-n) \Gamma)^{n}\left(U_{v z}-U_{\ell z}\right)^{n+1}
\end{aligned}
$$

and the wall friction terms are given by:

$$
\begin{aligned}
& M_{W z V}=f_{v}^{n} \cdot U_{v z}^{n} \cdot U_{v z}^{n+I} \\
& M_{W z \ell}=f_{\ell}^{n} \cdot U_{\ell z}^{n} \cdot U_{\ell z}^{n+1}
\end{aligned}
$$

A detalled description of the donor cell technique used to evaluate the terms $\Delta \mathrm{U}_{\mathrm{Vz}_{1+\frac{1}{2}} \mathrm{n}}$, etc. is given in Reference 1. Since these terms are treated explicitly while the main focus of this section is the treatment of the implicit variables, the technique used need not be repeated. The locations used to evaluate the terms are shown in figure A.1.

For greater clarity, equation A.1 can be rearranged so 


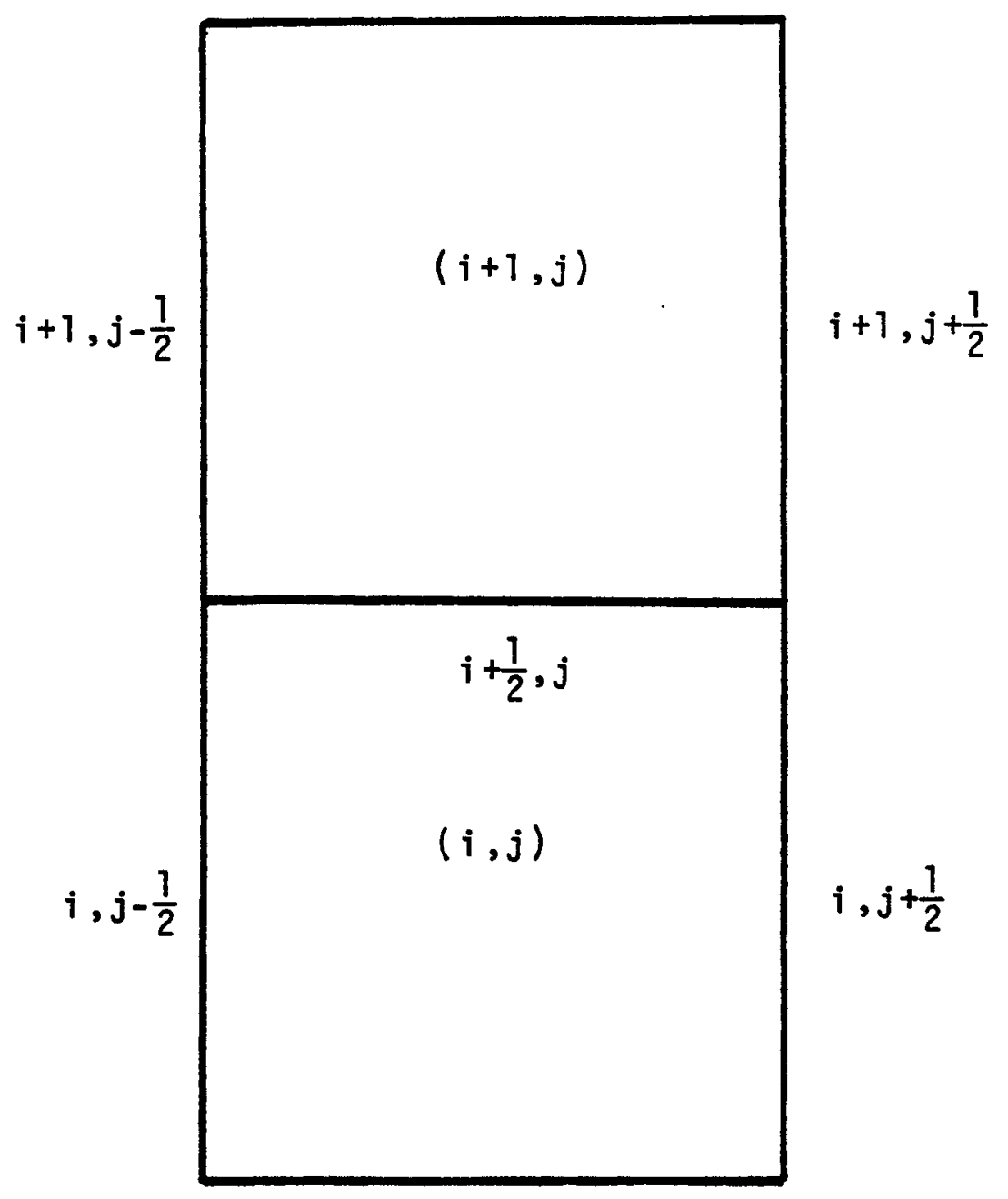

Figure A.1 Positions Used for the Evaluation of Variables 
that all implicit terms appear on the left hand side. When the relations given by equations $A .3$ and A.5 are substituted into equation $A .1$ the result is

$$
\begin{aligned}
& \left(\frac{\left(\alpha \rho_{v}\right)}{\Delta t}+f_{v} U_{v z}+(K+n \Gamma)\right)_{\substack{1+\frac{1}{2} j \\
U_{v z}}}^{n}+\alpha_{i+\frac{1}{2} j}^{n} \frac{\left(P_{i+1 j}^{n+1}-P_{i j}^{n+1}\right)}{\Delta z_{i+\frac{1}{2}}} \\
& -(K+n \Gamma) U_{\ell z i+\frac{1}{2} j}^{n+1}=\text { EXPLICIT TERMS }
\end{aligned}
$$

As can be seen, the specific choice of the time step discretinization has made the velocities only dependent on the pressures. Since there is also an equation corresponding to $A .7$ for the liquid phase, the term $U_{\ell z}^{n+1} 1+\frac{1}{2} j$ can be replaced with a term only dependent on $U_{V z}^{n+1}, P_{i+1 j}^{n+1}$, and $P_{i j}^{n+I}$. Thus knowledge of any two variables in equation A.7 allows the calculation of the third.

The iteration scheme for the solution of the eight conservation equatons ( 2 mass, 2 energy, and 4 momentum) is an extension of the Newton iteration solution of algebraic equations. The equations are cast in a form similar to that of $A .1$ and A.2, and then a vector $\vec{X}$ of the unknowns is defined such that

$$
\vec{X}=\left(\alpha, P, T, T_{V}, U_{V z}, U_{v r}, U_{\ell z}, U_{\ell r}\right)^{n+1}
$$

The finite difference equations can be written in the form

$$
F_{p}(\vec{x})=0
$$

where 


$$
p=1,2, \ldots 8
$$

At an iteration $k$, an approximate solution will be obtained. $F_{p}\left(\vec{x}^{k}\right)$ will not equal zero in general, so this is not an exact solution. A Taylor expansion around the point $x^{k}$ is made to obtain

$$
F_{p}\left(X^{k+1}\right)=F_{p}\left(X^{k}\right)+\left.\sum_{q=1}^{8} \frac{\partial F_{p}}{\partial X_{q}}\right|_{X_{q}} \cdot\left(X_{q}^{k+1}-X_{q}^{k}\right)
$$

If $x^{k+1}$ is required to be the solution of $A .9$ then

$$
\left.\sum_{q=1}^{8} \frac{\partial F_{p}}{\partial X_{q}}\right|_{X q} \cdot\left(X_{q}^{k+1}-X_{q}^{k}\right)=-F_{p}\left(X^{k}\right)
$$

Defining $\delta X=x_{q}^{k+1}-x_{q}^{k}$, equation $A .11$ can be written explicitly for the vapor and liquid momentum equations. The result is:

vapor phase

$$
\begin{aligned}
& \frac{\left(\alpha \rho_{v}\right)_{i+\frac{1}{2}} j \cdot \delta U_{v z}}{\Delta t}+\left(\frac{\alpha}{\Delta z}\right)_{1+\frac{1}{2} j} \cdot\left(\delta P_{1+1 j}-\delta P_{1 j}\right)+\frac{\partial M_{w z V}}{\partial U_{v z}} \cdot \delta U_{v z} \\
& +\frac{\partial M_{v \ell z}}{\partial U_{v z}} \cdot \delta U_{v z}+\frac{\partial M_{v \ell z}}{\partial U_{\ell z}} \cdot \delta U_{\ell z}=-F_{I}
\end{aligned}
$$

liquid phase

$$
\begin{aligned}
& \frac{\left((I-\alpha) \rho_{\ell}\right)}{\Delta t} 1+\frac{1}{2} j \cdot \delta U_{\ell z}+\left(\frac{1-\alpha}{\Delta z}\right)_{1+\frac{1}{2} j} \cdot\left(\delta P_{1+1 j}-\delta P_{1 j}\right)+\frac{\partial M_{W z \ell}}{\partial U_{\ell z}} \cdot \delta U_{\ell z} \\
& -\frac{\partial M_{\ell v z}}{\partial U_{\ell z}} \cdot \delta U_{\ell z}-\frac{\partial M_{\ell v z}}{\partial U_{v z}} \cdot \delta U_{v z}=-F_{2}
\end{aligned}
$$

Solving equations A.12 and A.13 for the velocities yields

$$
\delta U_{v z_{i+\frac{1}{2} j}}=W_{v z_{i+\frac{1}{2} j}}\left(\delta P_{1+1 j}-\delta P_{i j}\right)+R_{v z}
$$




$$
\delta U_{\ell z_{i+\frac{1}{2} j}}=W_{\ell z_{1+\frac{1}{2} j}}\left(\delta P_{1+1 j}-\delta P_{1 j}\right)+R_{\ell z}
$$

where

$$
\begin{aligned}
& \mathrm{W}_{\mathrm{vz}_{i+\frac{1}{2} j}}=-\left(\frac{\alpha}{\Delta z}\left(\frac{(1-\alpha) \rho_{\ell}}{\Delta t}+\frac{\partial \mathrm{M}_{\mathrm{wz} \ell}}{\partial U_{\ell z}}-\frac{\partial \mathrm{M}_{\ell v z}}{\partial U_{\ell z}}\right)-\frac{\partial \mathrm{M}_{\mathrm{V} \ell z}}{\partial U_{\ell z}} \cdot \frac{(1-\alpha)}{\Delta z}\right) \\
& \left(\left(\frac{\alpha \rho_{v}}{\Delta t}+\frac{\partial M_{w z V}}{\partial U_{v z}}+\frac{\partial M_{v \ell z}}{\partial U_{v z}}\right)\left(\frac{(1-\alpha) \rho_{\ell}}{\Delta t}+\frac{\partial M_{w z \ell}}{\partial U_{\ell z}}+\frac{\partial M_{\ell v z}}{\partial U_{\ell z}}\right)+\frac{\partial M_{v \ell z}}{\partial U_{\ell z}} \frac{\partial M_{\ell v z}}{\partial U_{v z}}\right)
\end{aligned}
$$

$\mathrm{R}_{\mathrm{vz}}=$

$$
\frac{F_{1} \cdot\left(\frac{(I-\alpha) \rho_{\ell}}{\Delta t}+\frac{\partial M_{w z \ell}}{\partial U_{\ell z}}+\frac{\partial M_{\ell v z}}{\partial U_{\ell z}}\right)-F_{2} \cdot \frac{\partial M_{V \ell z}}{\partial U_{\ell z}}}{\left(\left(\frac{\alpha \rho_{V}}{\Delta t}+\frac{\partial M_{w z V}}{\partial U_{v z}}+\frac{\partial M_{v \ell z}}{\partial U_{v z}}\right)\left(\frac{(1-\alpha) \rho_{\ell}}{\Delta t}+\frac{\partial M_{w z \ell}}{\partial U_{\ell z}}+\frac{\partial M_{\ell V z}}{\partial U_{\ell z}}\right)+\frac{\partial M_{v \ell} z}{\partial U_{\ell z}} \frac{\partial M_{\ell z}}{\partial U_{v z}}\right)}
$$

and similarly for the liquid phase.

Equation A.14 and A.15 are the form of the momentum equations which will be used in the following two sections to describe the inlet velocity and mass flow rate boundary conditions. 


\section{A.3 Inlet Velocity Boundary Condition}

An inlet velocity boundary condition refers to a user defined fluid velocity, given as a function of time, which is constant across the bundle inlet. Only the inlet velocity and the outlet pressure need to be specified, since the inlet pressure no longer enters into the calculation. With these two parameters, the iteration scheme can calculate the pressure field distribution. After the iteration is performed, the inlet pressure necessary to generate the specified velocity can be inferred. However, since a constant velocity across the bundle is assumed, in general there will be a different inlet pressure for each cell.

The inlet velocity condition is imposed by setting $W_{\mathrm{VZ}} 1+\frac{1}{2} j$ and $W_{\ell Z} i+\frac{1}{2} j$ in equation $A .14$ and $A .15$ to zero, and then setting

$$
\begin{aligned}
& R_{v z}=U_{\text {inlet }}-U_{v z 1+\frac{1}{2} j} \\
& R_{\ell z}=U_{\text {inlet }}-U_{\ell z 1+\frac{1}{2} \mathrm{j}}
\end{aligned}
$$

Since $R_{V Z}$ and $R_{\ell z}$ represent the error term from the previous iteration, one can see that as $R_{v z}$ and $R_{\ell z}$ go to zero, the Newton iteration converges on the exact solution. Equation A.7 is then used to update the boundary pressure, $P_{1 j}$, since $P_{2, i}, U_{V Z} I+\frac{1}{2} q$, and $U_{\ell Z} I+\frac{1}{2} ;$ are known.

The inlet velocity boundary condition calculation is 


\section{$-166-$}

simple to perform, but it has the disadvantage of preventing localized flow reversal during sodium bolling transients. The second method, inlet mass flow rate, does not have this restriction. 
A. 4 Specified Inlet Mass Flow Rate Boundary Condition

The method for specifying the inlet mass flow rate was developed by Andrei L. Schor at the Massachusetts Institute of Technology. For the cell numbering scheme shown in figure A.2, equations $A .1$ and A.2 can be written for the bottom row of real cells in the form:

$$
\begin{aligned}
& U_{\ell j}=a_{\ell j} \cdot\left(P_{j}-P_{0}\right)+r_{\ell j} \\
& U_{v j}=a_{v j} \cdot\left(P_{j}-P_{0}\right)+r_{v j}
\end{aligned}
$$

$$
j=1, \ldots n j
$$

The mass flow rate of each phase into the cell is given by:

$$
\begin{aligned}
& W_{\ell j}=A_{f} \cdot(I-\alpha) \rho_{\ell} U_{\ell j} \\
& W_{v j}=A_{j} \cdot \alpha \cdot \rho_{v} U_{v j}
\end{aligned}
$$

where

$$
\begin{aligned}
& W_{\ell j}=\text { liquid mass flow rate into cell } j \\
& W_{v j}=\text { vapor mass flow rate into cell } j \\
& A_{j}=\text { flow area of cell } j
\end{aligned}
$$

The total mass flow rate into the cell, $\mathrm{w}_{j}$, is:

$$
\begin{aligned}
w_{j}= & w_{\ell j}+w_{v j} \\
w_{j}= & A_{j}(1-\alpha) \rho_{\ell}\left(a_{\ell j}\left(P_{j}-P_{0}\right)+r_{\ell j}\right)+ \\
& A_{j} \alpha \rho_{v}\left(a_{v j}\left(P_{j}-P_{0}\right)+r_{v j}\right)
\end{aligned}
$$




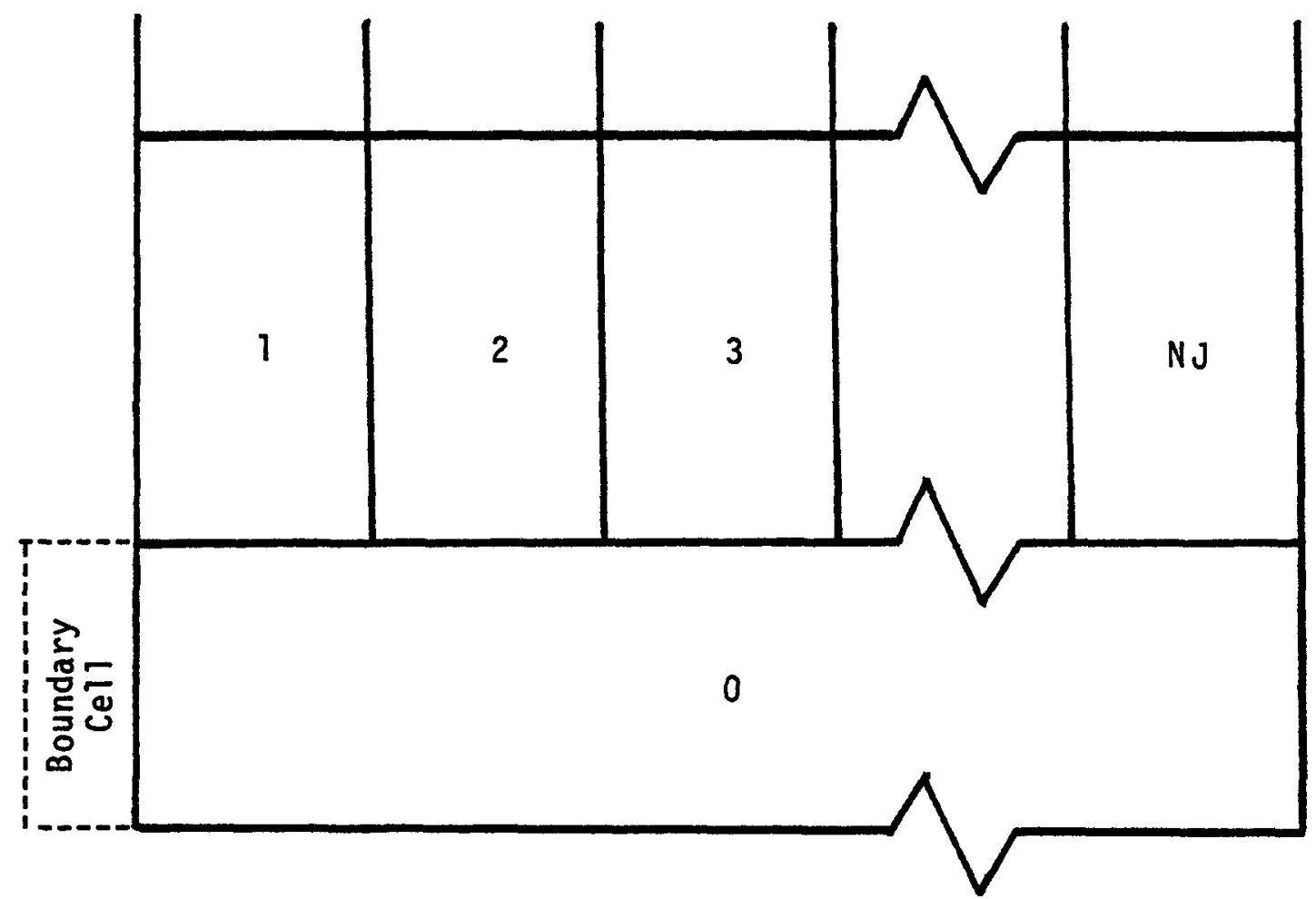

Figure A.2 Example Cell Numbering Scheme for Flow Boundary Calculation 


$$
\begin{aligned}
W_{j}= & A_{j}\left((1-\alpha) \rho_{\ell} a_{\ell j}+\alpha \rho_{v} a_{v j}\right)\left(P_{j}-P_{0}\right)+ \\
& A_{j}\left((1-\alpha) \rho_{\ell} r_{\ell j}+\alpha \rho_{v} r_{v j}\right)
\end{aligned}
$$

And the total mass flow rate into the bundle then becomes:

$$
\mathrm{w}_{\mathrm{T}}=\sum_{j=1}^{n j} \mathrm{w}_{j}
$$

Defining

$$
\begin{aligned}
& a_{j}^{\prime}=A_{j}\left((1-\alpha) \rho_{\ell} a_{\ell j}+\alpha \rho_{v} a_{v j}\right) \\
& r_{j}^{\prime}=A_{j}\left((1-\alpha) \rho_{\ell} r_{\ell j}+\alpha \rho_{v} r_{v j}\right)
\end{aligned}
$$

$\mathrm{W}_{\mathrm{T}}$ is then given by:

$$
\begin{aligned}
w_{T} & =\sum_{j=1}^{n j} a_{j}^{\prime}\left(P_{j}-P_{0}\right)+\sum_{j=1}^{n j} r_{j} \\
& =\sum_{j=1}^{n j} a_{j}^{\prime} P_{j}-P_{0} \sum_{j=1}^{n j} a_{j}^{j}+\sum_{j=1}^{n j} r_{j}
\end{aligned}
$$

Rearranging equation A.30 yields

$$
\left(-\sum_{j=1}^{n j} a_{j}^{\prime}\right) P_{0}+a_{1}^{\prime} P_{I}+\cdots+a_{n j}^{\prime} P_{n j}=W_{T}-\sum_{j=1}^{n j} r_{j}^{\prime}
$$

When written in incremental form, equation A.31 becomes

$$
\left(-\sum_{j=1}^{n j} a_{j}^{\prime}\right) \delta P_{0}+a_{1}^{\prime} \delta P_{1}+a_{2}^{\prime} \delta P_{2}+\cdot \cdot a_{n j}^{\prime} \delta P_{n j}=0
$$

Thus, for the specified mass flow rate boundary condition, there will be an additional pressure field equation to solve. This equation is added to the pressure field matrix described in Chapter 4. For the configuration of cells shown in figure A.3, the resulting matrix is shown in figure A. 4 .

The coefficients $c_{01}, c_{02}$ and $c_{03}$ are given by equation 


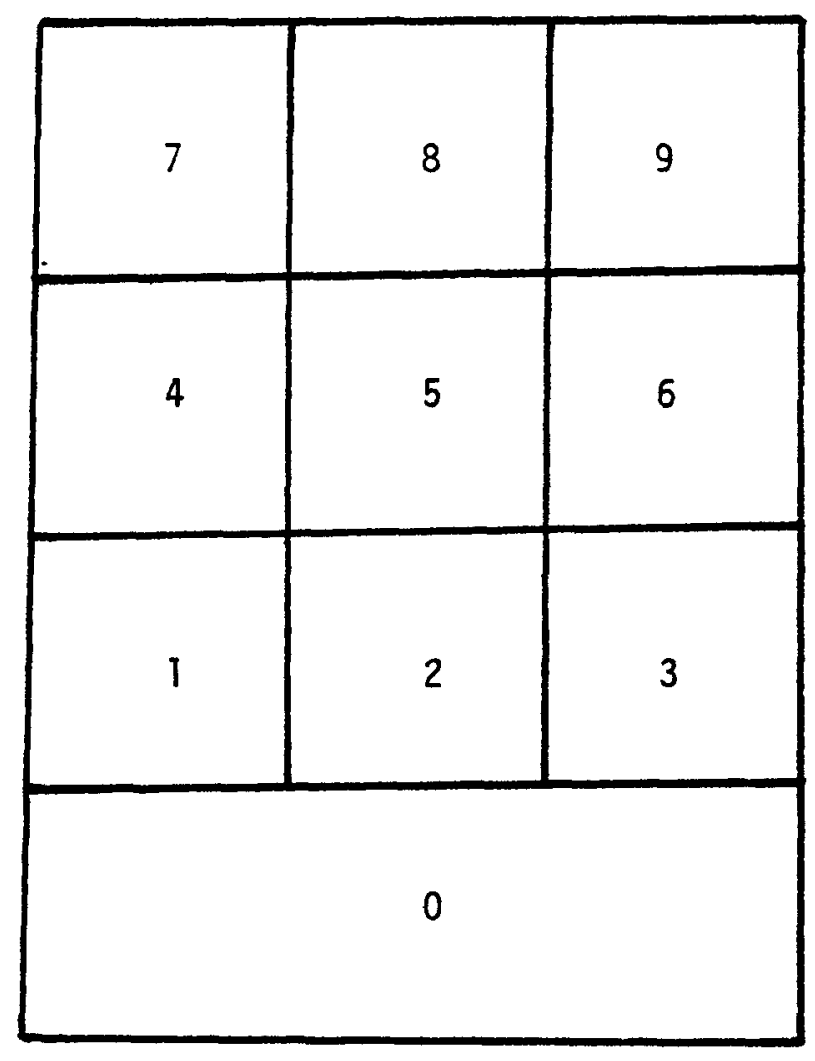

Figure A.3 Cell Configuration for Matrix Shown in Figure A.4 


$\begin{array}{llllllllll}1 & c_{01} & c_{02} & c_{03} & 0 & 0 & 0 & 0 & 0 & 0 \\ c_{10} & a_{1,1} & a_{1,2} & 0 & a_{7,4} & 0 & 0 & 0 & 0 & 0 \\ c_{20} & a_{2,1} & a_{2,2} & a_{2,3} & 0 & a_{2,5} & 0 & 0 & 0 & 0 \\ c_{30} & 0 & a_{3,2} & a_{3,3} & 0 & 0 & a_{3,6} & 0 & 0 & 0 \\ 0 & a_{4,1} & 0 & 0 & a_{4,4} & a_{4,5} & 0 & a_{4,7} & 0 & 0 \\ 0 & 0 & a_{5,2} & 0 & a_{5,4} & a_{5,5} & a_{5,6} & 0 & a_{5,8} & 0 \\ 0 & 0 & 0 & a_{6,3} & 0 & a_{6,5} & a_{6,6} & 0 & 0 & a_{6,9} \\ 0 & 0 & 0 & 0 & a_{7,4} & 0 & 0 & a_{7,7} & a_{7,8} & 0 \\ 0 & 0 & 0 & 0 & 0 & a_{8,5} & 0 & a_{8,7} & a_{8,8} & a_{8,9} \\ 0 & 0 & 0 & 0 & 0 & 0 & a_{9,6} & 0 & a_{9,8} & a_{9.9}\end{array}$

Figure A.4 Pressure Field Matrix with Flow Boundary Calculation 
A.8. The coefficients $c_{10}, c_{20}$ and $c_{30}$ are the coefficients of the momentum equation at the boundary. Previously, these terms were not used, since the boundary pressure was constant during a timestep. With the flow boundary condition, however, these terms are used to relate the pressure in the boundary cell to the bottom row of real cells, and in this way the boundary cell pressure can be updated.

Note that the bandwidth of the matrix remains the same, and therefore the additional cpu requirements are negligible.

This method offers an advantage over a specified inlet velocity, since it allows the boundary pressure to adjust itself to the conditons prevalent in the bundle. Thus, in principle, it is possible to have flow reversal in some channels, while still maintaining a net positive flow. 


\section{A.5 Programming Information}

The boundary condition at the inlet is specified by setting the input parameter, itbd, to $-1,0$ or 1 to indicate a velocity, pressure, or flow boundary condition.

For the velocity boundary condition, the velocity at the inlet is input, and the velocities are updated in subroutine $B C$. These values are passed to subroutine ONESTP, where the differnece between $U_{b}$ and $U_{j}(i=1, n j)$ is used to calcualte $\Delta U$, rather than $\Delta P$ in the boundary cell momentum equations.

For the flow boundary condition, the desired flow input for the timestep is used along with the pressures of the previous timestep to get a first estimate for $P_{0}$ in subroutine $B C$ (that is, solving equation $A .31$ for $P_{0}$ ). The new $P_{0}$ is passed to subroutine ONESTP, where it is treated the same as a pressure boundary condition. The difference is that $\delta P_{0}$ is calculated in subroutine DIRECT, and added to $P_{0}$ to get a better estimate. This is continued until convergence is attained. This option is only available when the direct method pressure field solution is used. 
Appendix B

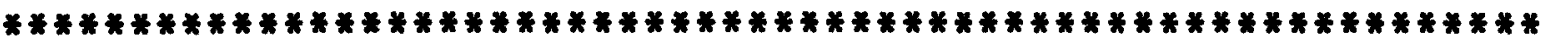

********** N A T 0 F -2 D - INPUT DESCRIPTION

$* * * * * * * * * *$

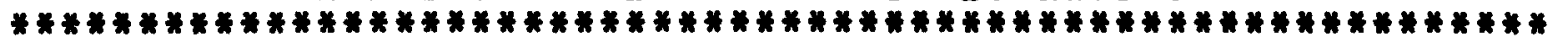

\section{SECTION I}

The following cards are read via namelist input. A total of four namelists are used: "restrt", "unos", "duos", and "tres".

The input should look like: $\$$ "namelistn $f 1, f 2, f 3, \ldots, f n$, \$end

for each namelist, where each fI is a field consisting of: all blanks, or

name = constant, or

name = list of constants.

The order of input is immaterial; as many cards as needed may be used; the \$end signifying the end of the namelist input should appear only on the last card, for each namelist.

For additional details on the use of namelist input, the user is referred to a standard fortran manual.

Group

No.

Format

Contents

1

namelist

\$restrt, nres , \$end

nres = steady state or transient indicator $(1 / 0)$

2

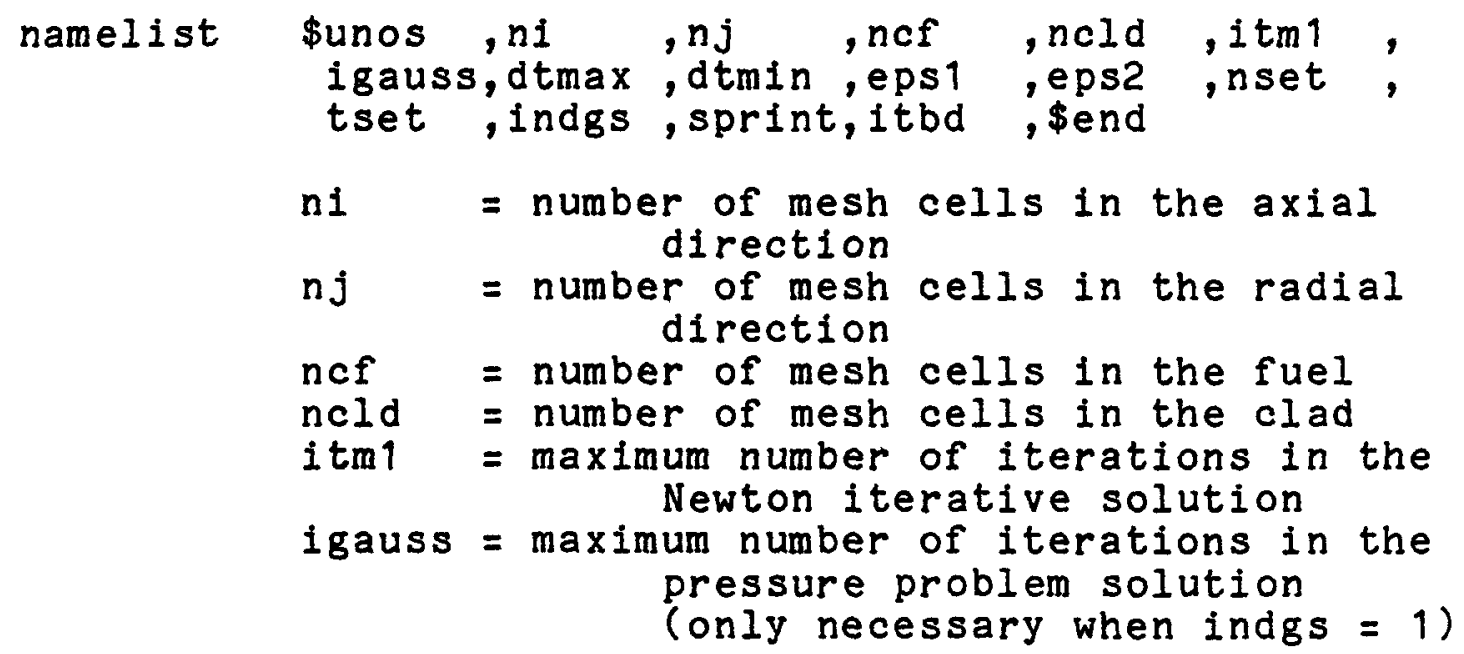




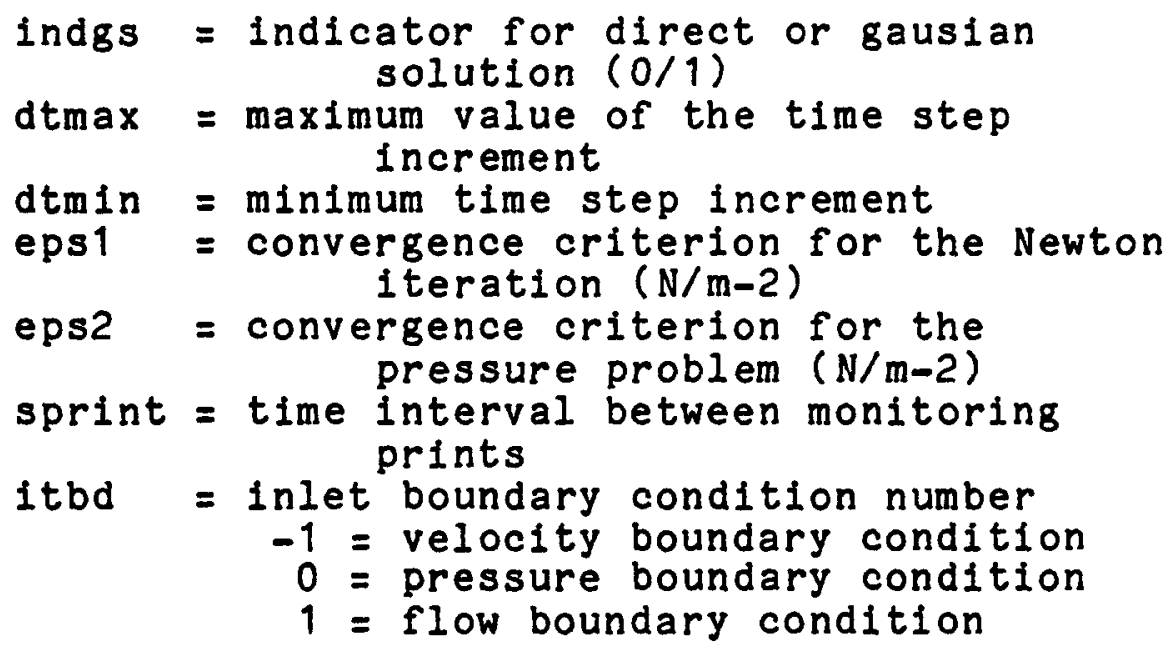

The following two corresponding cards can be incremented from $1=1$ to 40 , and control

the printed output. The code will print nset times the flow map at an increment of tset.

nset(1) $=$ number of printouts

tset $(1)=$ time between printouts

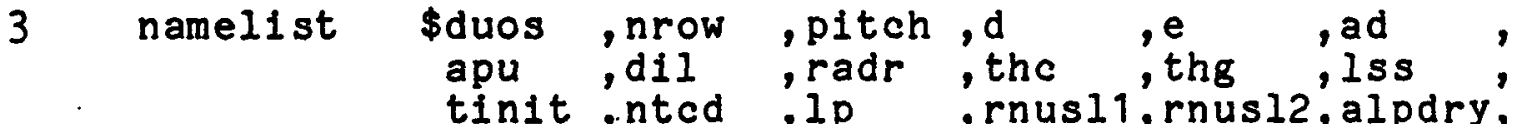
tinit, nted, ip , rnusl1, rnusl2, alpdry,

nrow = number of rows of fuel pins in the fuel assembly

pitch = distance between fuel pin centerlines $(\mathrm{m})$

d = diameter of the fuel pin (m)

e = minimum distance between fuel pin surface and hex can wall (m)

ad = fraction of theoretical density of fuel

apu = fraction of plutonium in the fuel

dil = fraction of helium gas in gas compositon

radr = fuel pin outside radius (m)

the $=$ clad thickness ( $\mathrm{m}$ )

thg = gap thickness (m)

iss = transient or steady state indicator $(0 / 1)$

tinit = inftial starting time ( $\mathrm{sec}$ )

ntcd = number of boundary condition cards

Ip = partial or full boundary calculation $(0 / 1)$

rnusll = effective nusselt number for radial 


$$
\begin{gathered}
\text { heat conduction - inner cells } \\
0>\text { implicit calculation } \\
0 \text { = bypass calculation } \\
0 \text { < explicit calculation } \\
\text { rnusl2 = effective nusselt number for radial } \\
\text { heat conduction - edge cells } \\
\text { alpdry = dryout vold fraction }
\end{gathered}
$$

The following cards are required only for an initial start, and appear at the very end of the input file.

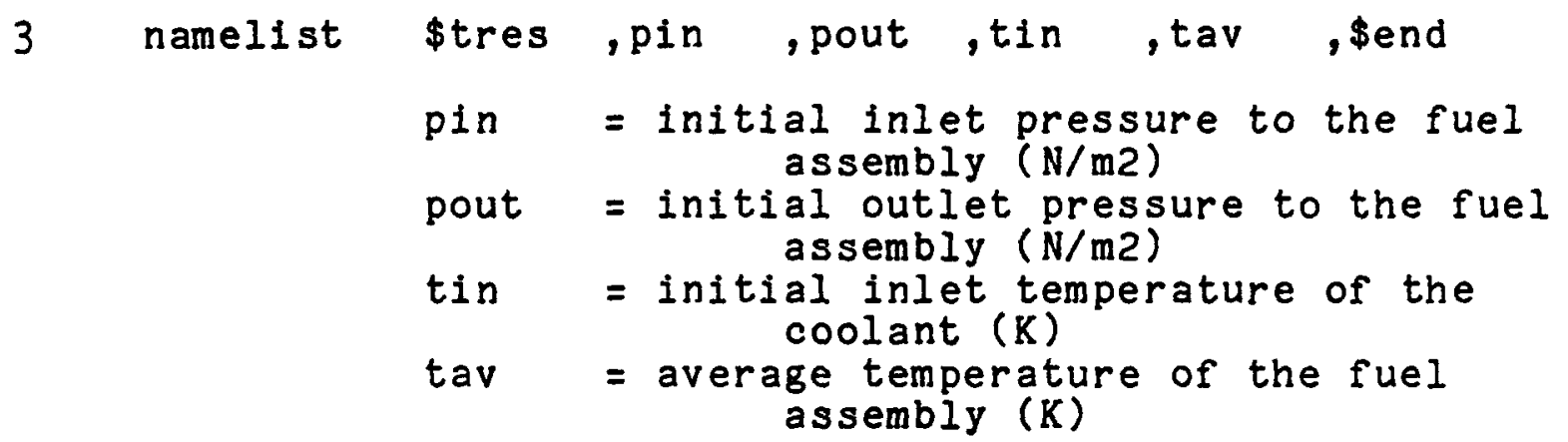

\section{SECTION II}

The following cards are read via NIPS free-format input processor. Fields are separated by blanks. Entry (or group of entries) repetition is allowed; for example $n(a b m(c d e) f)$ where: $a, b, c, d, e, f$ are entries (integer or real) and $n, m$ are integers representing the number of repetitions; note that no blanks must appear between a left parenthesis and the integer preceding it. Up to 10 levels of nesting are permitted.

The end of a group is marked by a $\$-s i g n$.

The following cards govern the boundary conditions of the problem as a function of time. These cards are always required. The order of input must be maintained. Those cards marked with a are necessary only for a full boundary calculation ( $1 \mathrm{p}=1)$

For a partial boundary calculation $(1 p=0)$, the boundary is calculated as follows:

$$
X=X 1(L) * d t i m e+X 2(L)
$$

and for a full boundary calculation:

$$
\begin{aligned}
X= & (X 1(L) * d t i m e+X 2(L)) * \exp (O M X(L) * d t I m e) \\
& +X 3(L)
\end{aligned}
$$


where

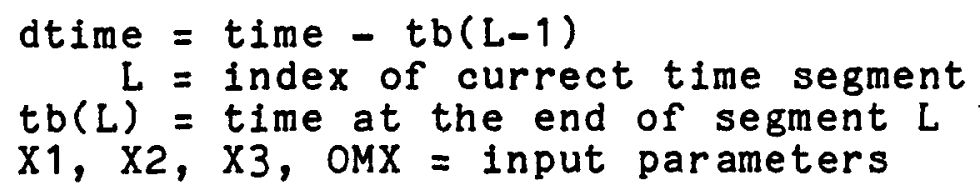

4 alb1(ntcd) = void fraction at the inlet of the fuel assembly

$\operatorname{alb2}($ ntcd $)=$
alb3(ntcd) $=$
oma(ntcd) $=$

5 trbl(ntcd) = vapor temperature at the inlet $(K)$ trb2 (ntcd) $=$

* $\operatorname{trb3}(n t c d)=$

* $\operatorname{omv}(n t c d)=$

6 tlb1(ntcd) = liquid temperature at the inlet( $K)$ $\operatorname{tlb} 2$ (ntcd) $=$

* $\operatorname{tlb} 3($ ntcd) $=$

* oml(ntcd) $=$

7 hnb1(ntcd) = power density in the fuel pins (w/m2) hnb2(nted) =
* $\operatorname{hnb} 3(n t c d)=$
* $\operatorname{omh}($ ntcd) $=$

The following cards are always required for a steady state calculation, but not for a transient calculation. The dimensions are given by:

$$
\begin{aligned}
& n i=\text { number of axial cells } \\
& n j=\text { number of radial cells } \\
& n p i n=n c f+n c l d+2 \\
& n n=n i * j
\end{aligned}
$$

$8 \mathrm{n}(19)=$ row numbers where the boundary 
between cell $\mathrm{J}$ and cell $\mathrm{J}+1$ lies $(n=2, \ldots)$

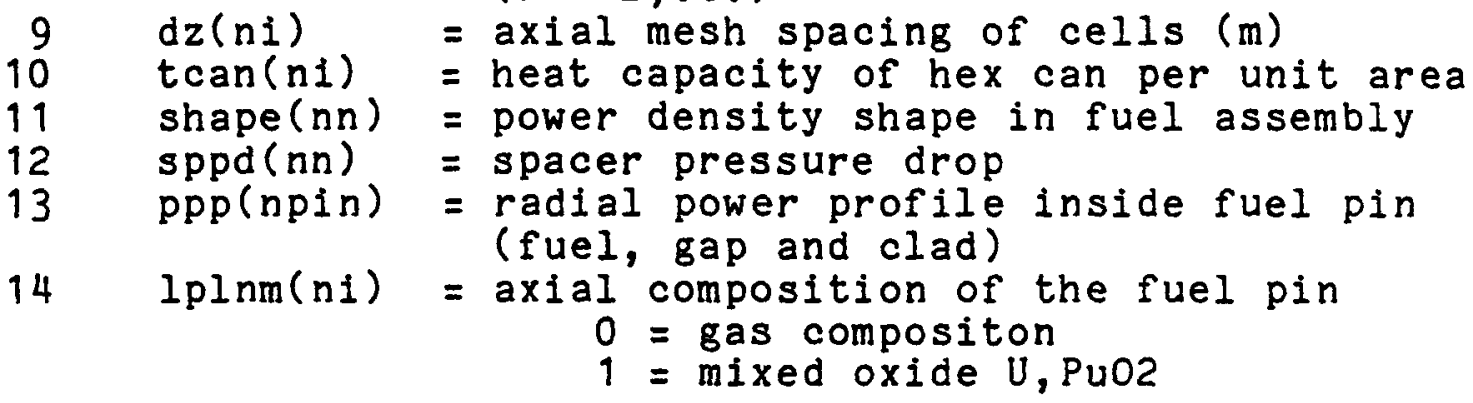

RESTART OPTION

For a restart of a previous calculation, the namelists restrt, unos and duos are required, for a selected number of cards.

For namelist restrt, the following previously defined card is required:

$$
\text { nres }=0 \quad(
$$

For namelist unos, the following previously defined cards are required:

$$
\begin{aligned}
& n \operatorname{nset}(i)= \\
& \operatorname{tset}(i)=
\end{aligned}
$$

The following cards are optional inputs:

$$
\begin{aligned}
& \text { eps1 ,eps2, dtmax , igauss, itm1, } \\
& \text { dtmin , sprint,itbd, indgs }
\end{aligned}
$$

For namelist duos, the following previously defined cards are required

$$
\begin{aligned}
& \text { Iss }=0 \\
& \text { tinit }= \\
& \text { nted }= \\
& \text { Ip }= \\
& \text { rnusil }= \\
& \text { rnusl2 }= \\
& \text { alpdry }=
\end{aligned}
$$

Boundary condition cards are always required.

As of September 1, 1981 
$\begin{aligned} \text { Appendix C.I: } & \text { Westinghouse Blanket Heat Transfer } \\ & \text { Test Program Run } 544\end{aligned}$

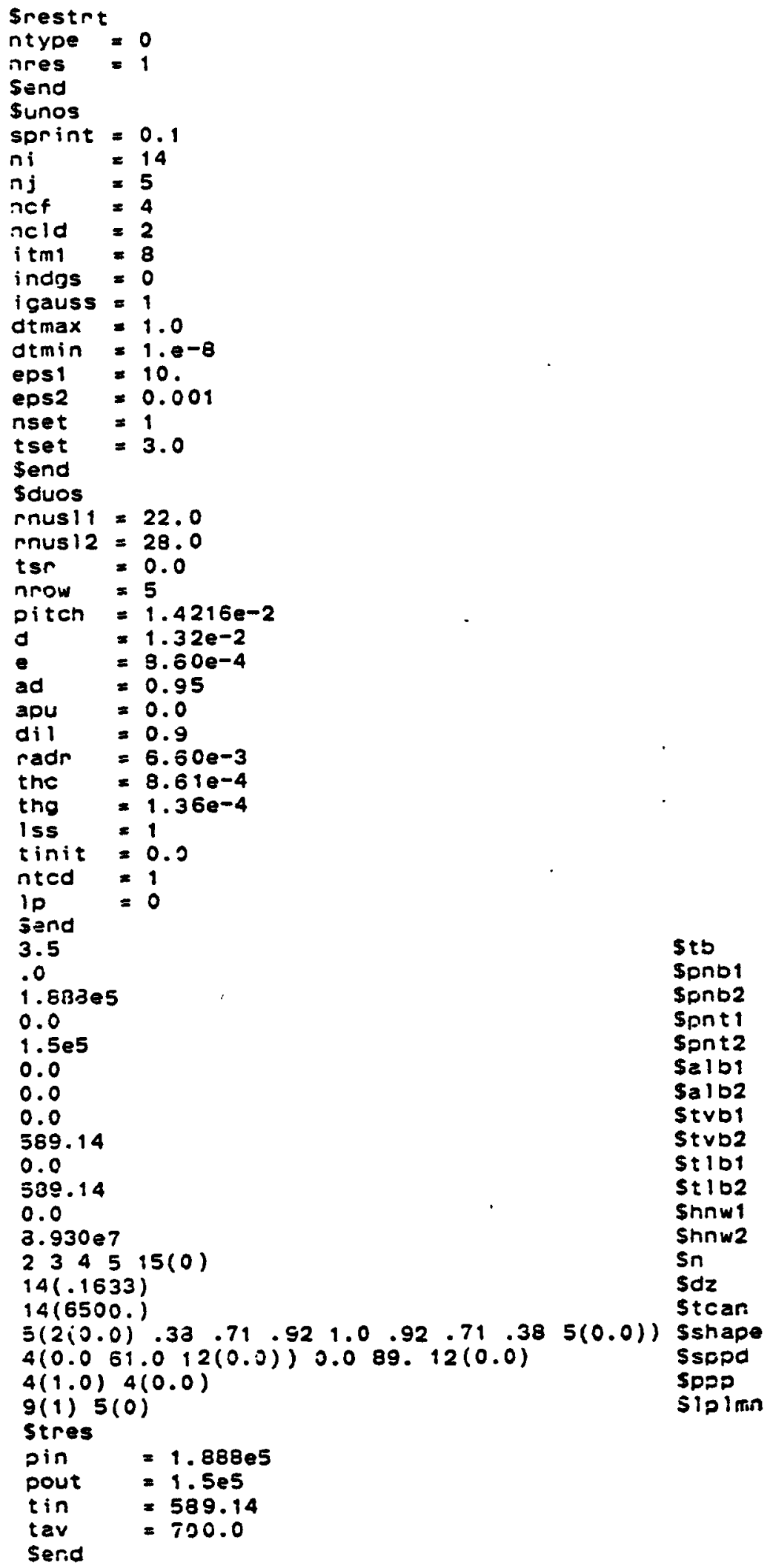


Appendix C.2: Westinghouse Blanket Heat Transfer

Srestrt
ntype

Test Program Run 545

Sen

Sunos

sprint $=0.1$

$\mathrm{ni}=14$

$n j=5$

nef $=4$

nold $=2$

$i \operatorname{tm} 1=8$

indgs $=0$

igaus $=100$

dimax $=1.0$

dtmin $=1.0$

eps1 $=10$.

eps $2=.001$

nset $=1$

tset $=3.0$

Send

Souos

rnus $11=-22.0$

rnus $12=-23.0$

tsr $=0.0$

nrow $=5$

pitch $=1.4216 \mathrm{e}^{-2}$

d $\quad=1.32 \mathrm{e}^{-2}$

e $\quad=8.60 \mathrm{e}-4$

ad $=0.95$

apu $=0.0$

dil $=0.9$

radr $=6.60 e^{-3}$

the $=8.61 \mathrm{e}-4$

tho $=1.36 e-4$

iss $=1$

tinit $=0.0$

ntcd $=1$

ip

Send

3.5

0.0

$1.835=5$

Stb

0.0

$1.5 e 5$

Spnb 1

0.0

0.0

0.0

589.14

0.0

589.14

0.0

$8.3755 e 7$

$234515(0)$

$14(.1633)$

$14(6500)$

$\begin{array}{lllllllll}2(0.0) & .31 & .58 & .76 & .82 & .76 & .58 & .31 & 5(0.0)\end{array}$

$\begin{array}{lllllllll}2(0.0) & .34 & .64 & .83 & .91 & .83 & .64 & .34 & 5(0.0)\end{array}$

$\begin{array}{lllllllll}2(0.0) & .39 & .72 & .93 & 1.02 & .93 & .72 & .39 & 5(0.0)\end{array}$

$2(0.0) .42 .79 \quad 1.02 \quad 1.11 \quad 1.02 \quad .79 .425(0.0)$

$\begin{array}{llllllllll}2(0.0) & .46 & .87 & 1 .: 2 & 1.22 & 1.12 & .87 & .46 & 5(0.0)\end{array}$

$5(0.061 .012(0.0))$

$4(1.0) 4(0.0)$

$9(1) 5(0)$

Stres

pin $=1.838$ e 5

pout $=1.5 \mathrm{es}$

$\operatorname{tin}=539.14$

tav $=700.0$

Send 
Appendix C.3: THORS Bundle 6A Test $71 \mathrm{H}$ Run 101 - Steady State

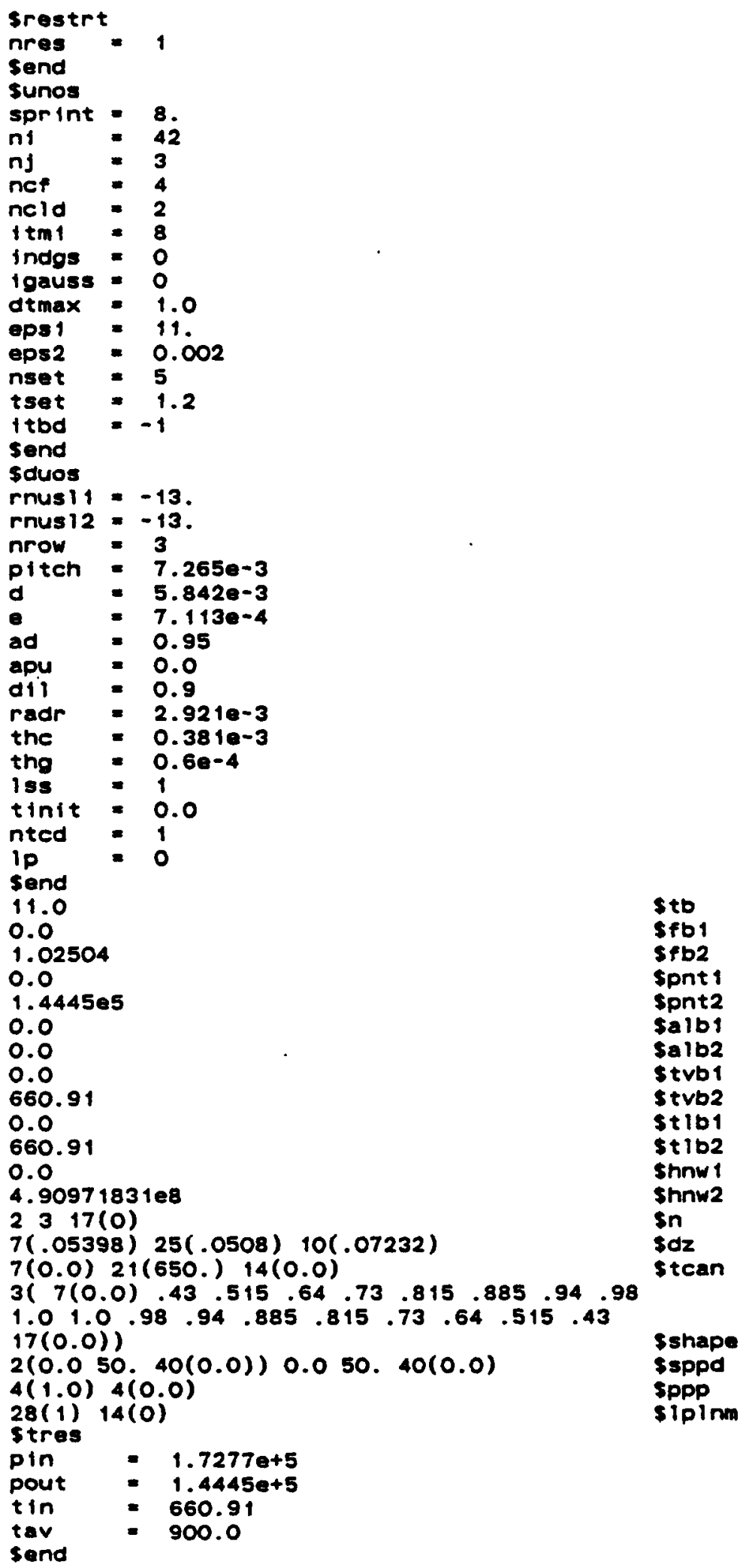


THORS Bundle $6 \mathrm{~A}$ Test $71 \mathrm{H}$ Run 101

- Transient $(0.0-11.0 \mathrm{sec})$

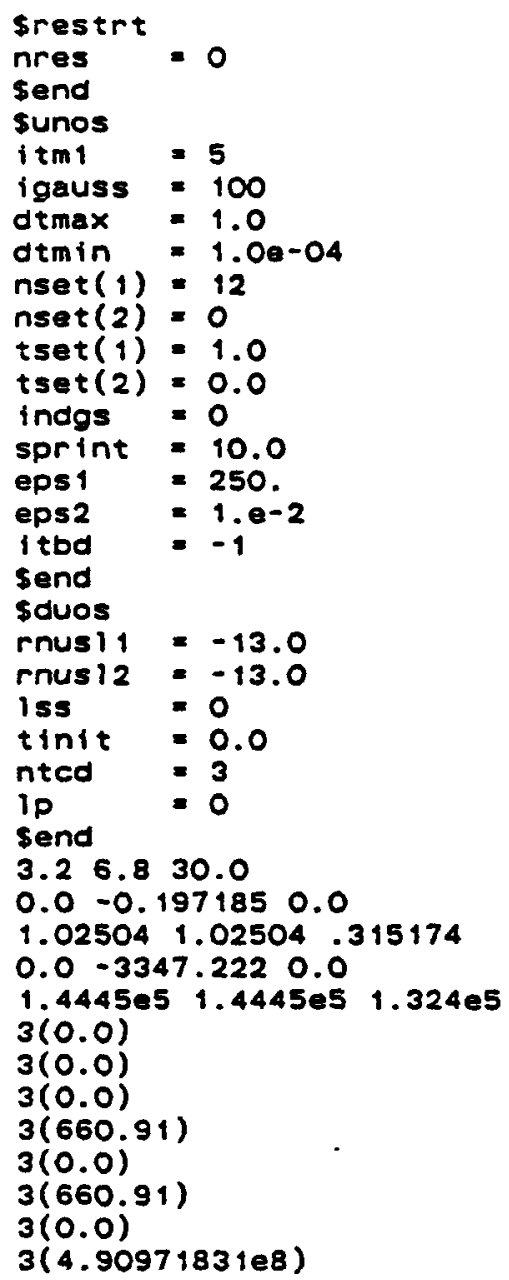

stb

sfbi

Sfb2

Spnt 1

Spnt2

Salbi

Salb2

Stvb 1

stvb2

$\$+101$

stib2

Shnb 1

Shnb2 
THORS Bundle $6 \mathrm{~A}$ Test $71 \mathrm{H}$ Run $10 \mathrm{I}$

- Transient (11.0 - $20.0 \mathrm{sec}$ )

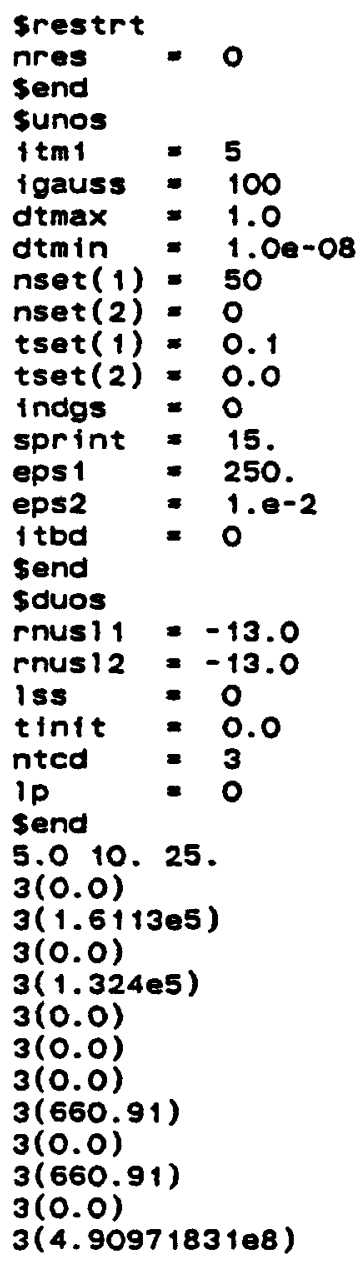

$\$$ tb Spnb 1 Spnb2 spnt 1 spnt2 Salb 1 Salb2 Stvb1 5 tvb2 $\$ t|b|$ $\$+1 b 2$ Shnb1 shnb2

The flow map output for this problem at Time $=11.3415 \mathrm{sec}$ appears in Appendix F. 
Appendix C. 4: W-I SLSF LOPI $2 \mathrm{~A}$

- Steady State

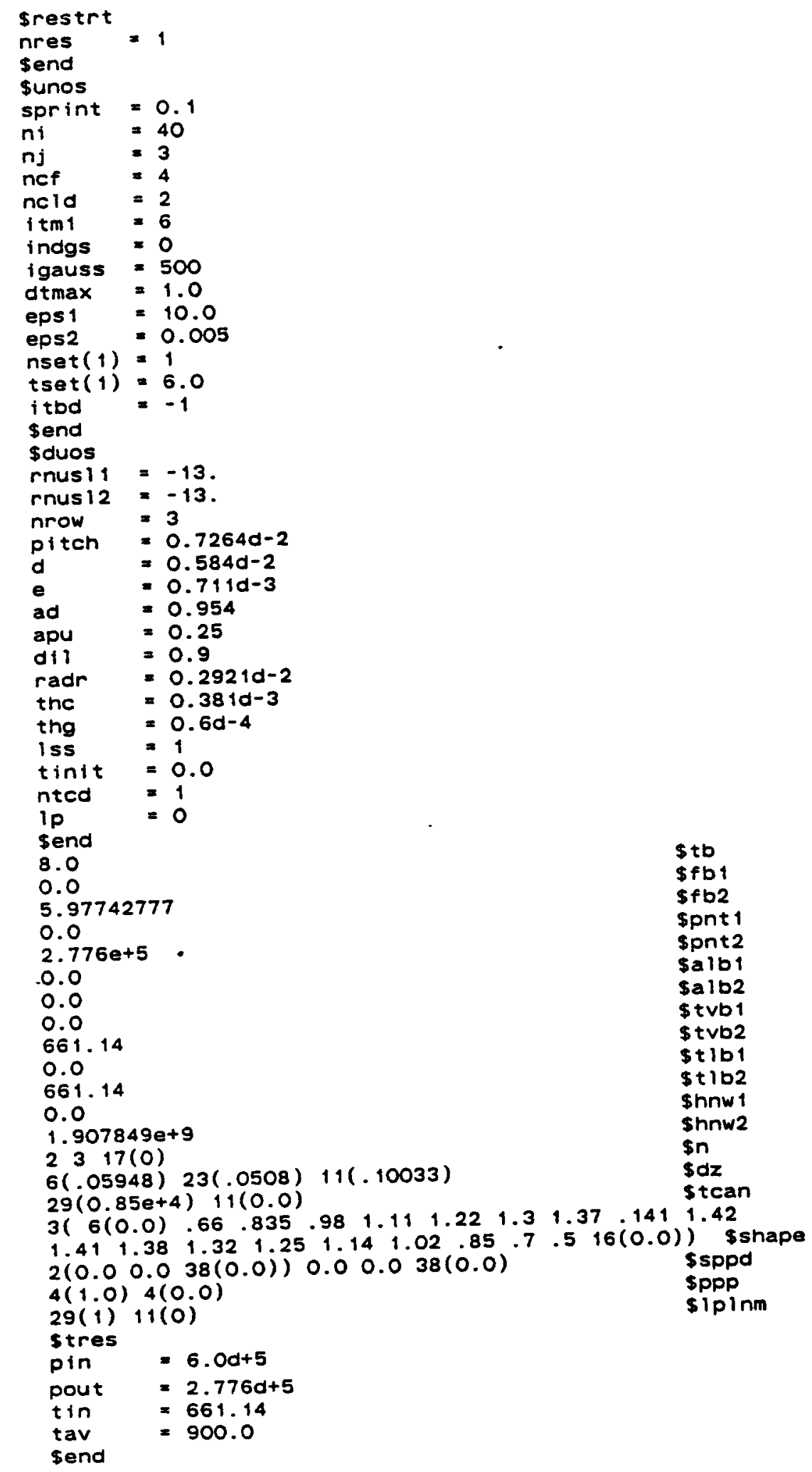


W- 1 SLSF LOPI $2 \mathrm{~A}$

- Transient $(0.0-4.5$ sec)

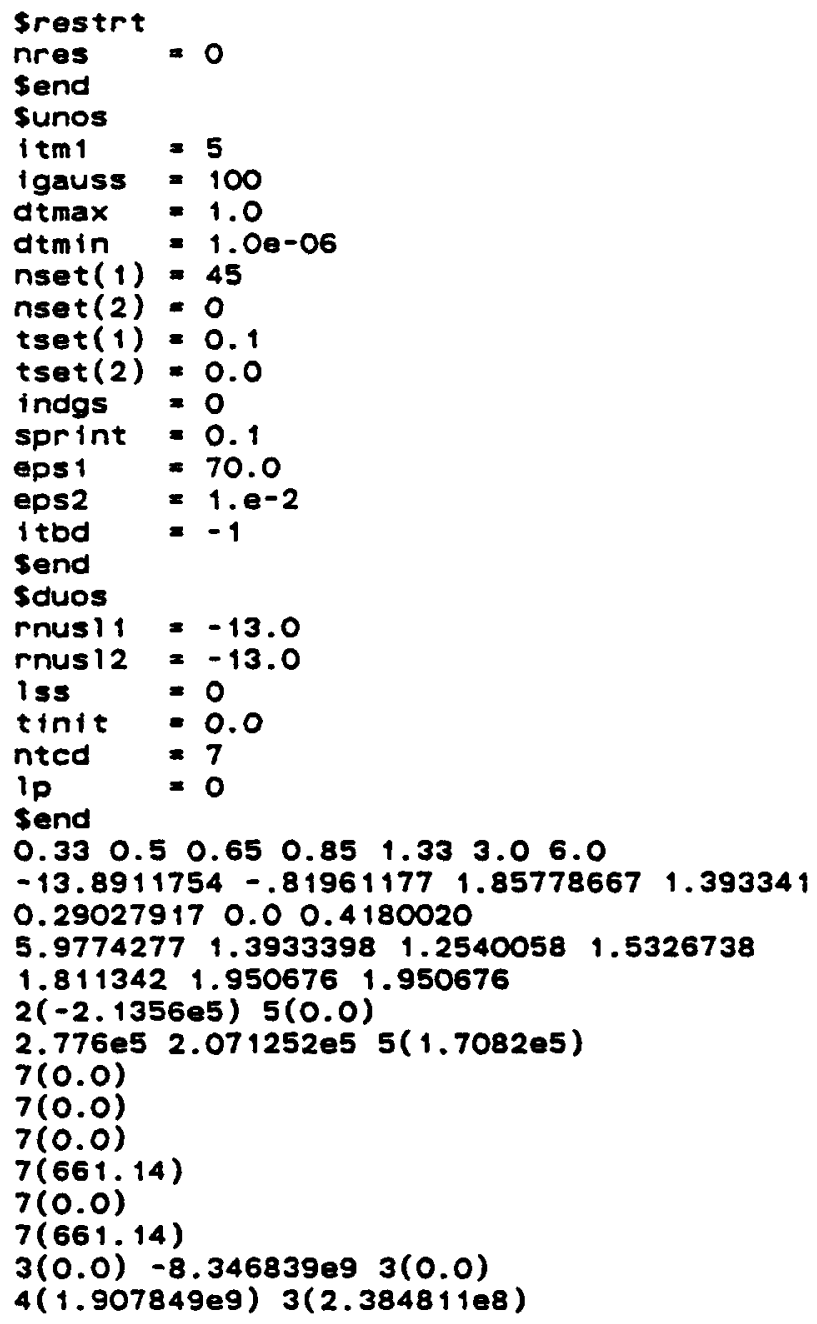

\$tb

svb 1

Svb 1 Spnt 1 Spnt2 Salbi $\$ a 1 b 2$ Strb1 stvb2 stib1 st $1 b_{2}$ Shnb1 shnb2 
Appendix C.5: W-1 SLSF LOPI 4

- Steady State

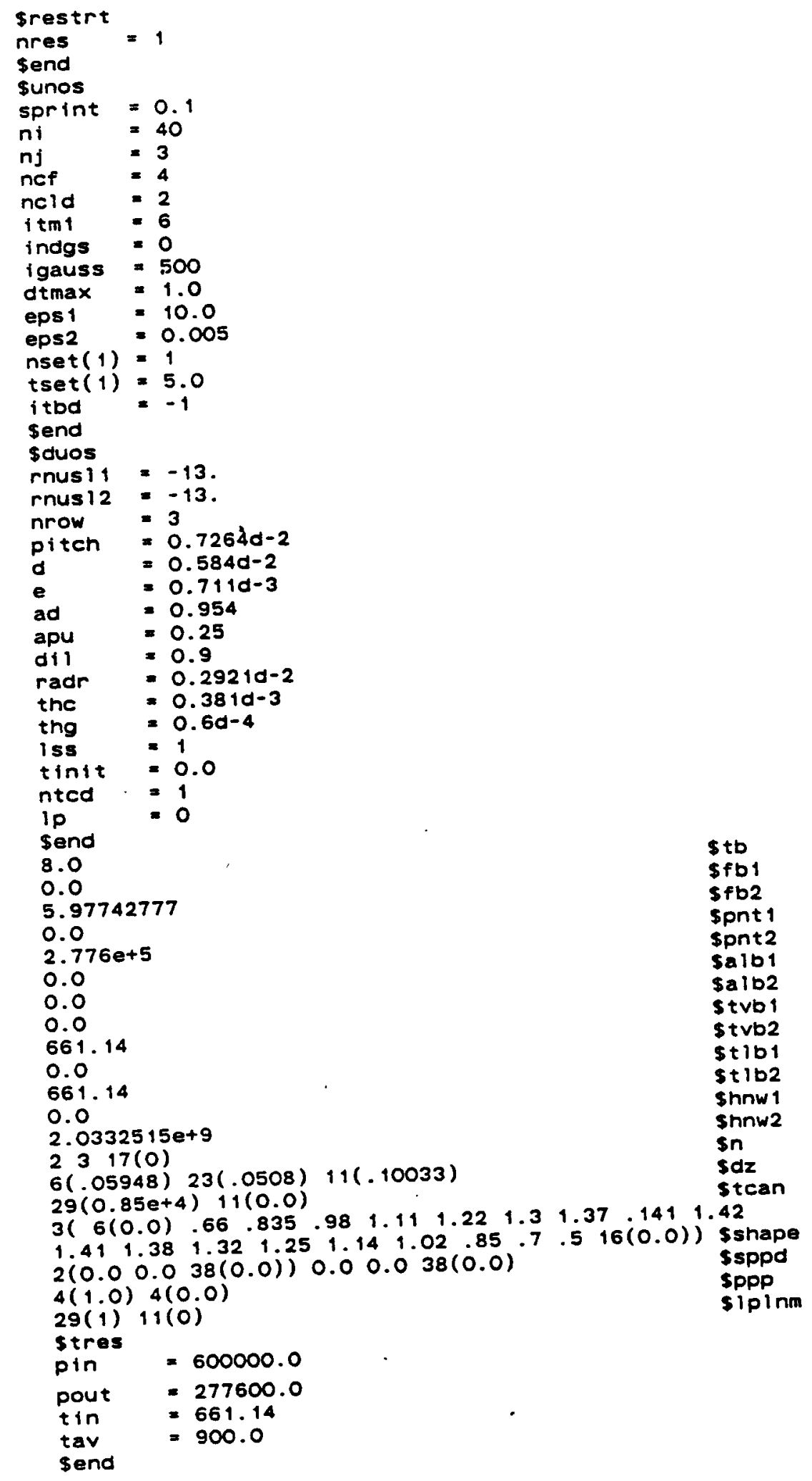


W-I SLSF LOPI 4

- Transient $(0.0-5.0 \mathrm{sec})$

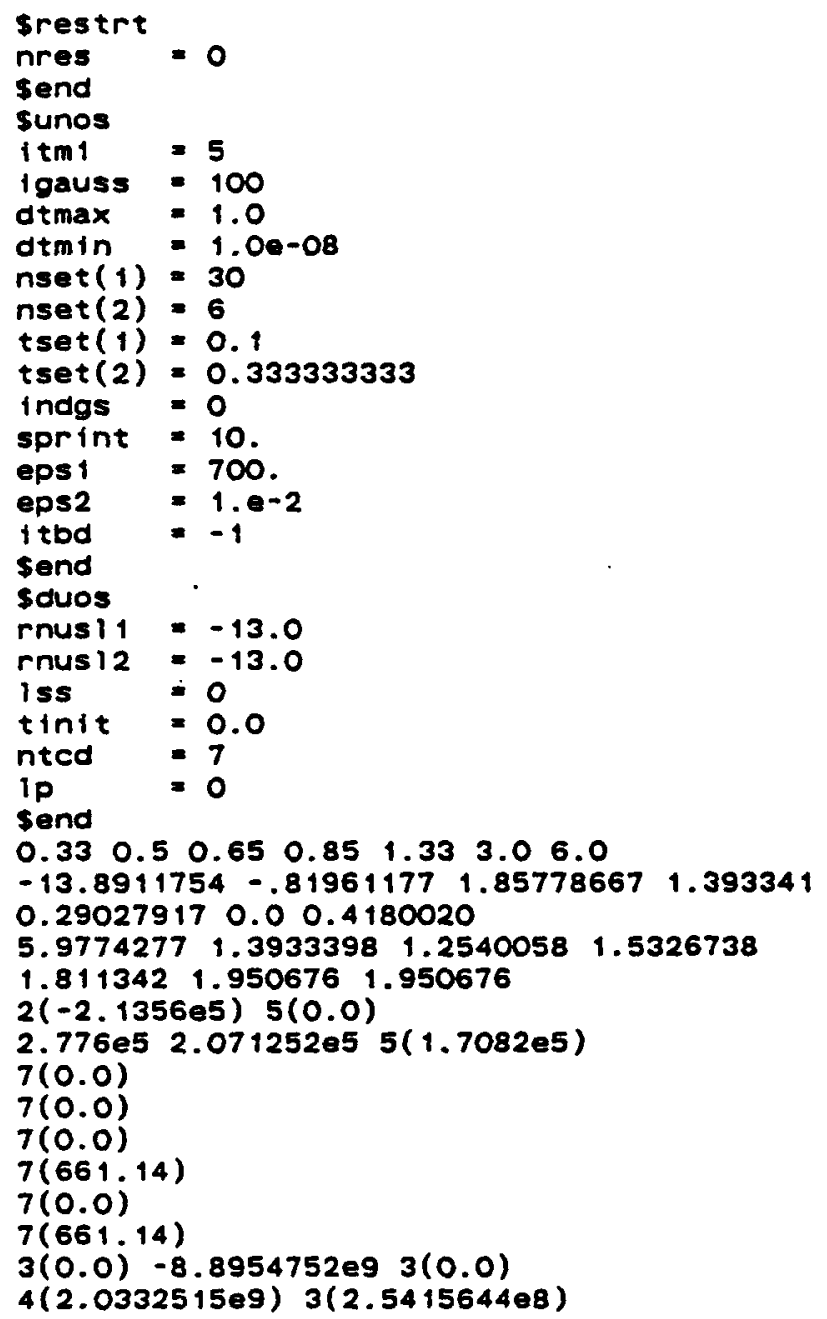

$s+b$

\$Vb 1

Svb1 Spnt 1 Spnt2 Salb1 $\$ a 1 b 2$ Stvb1 stvb2 stlo1 st 162 Shnol Shnb2 
Appendix C.6: W-1 SLSF BWT 2'

- Steady State

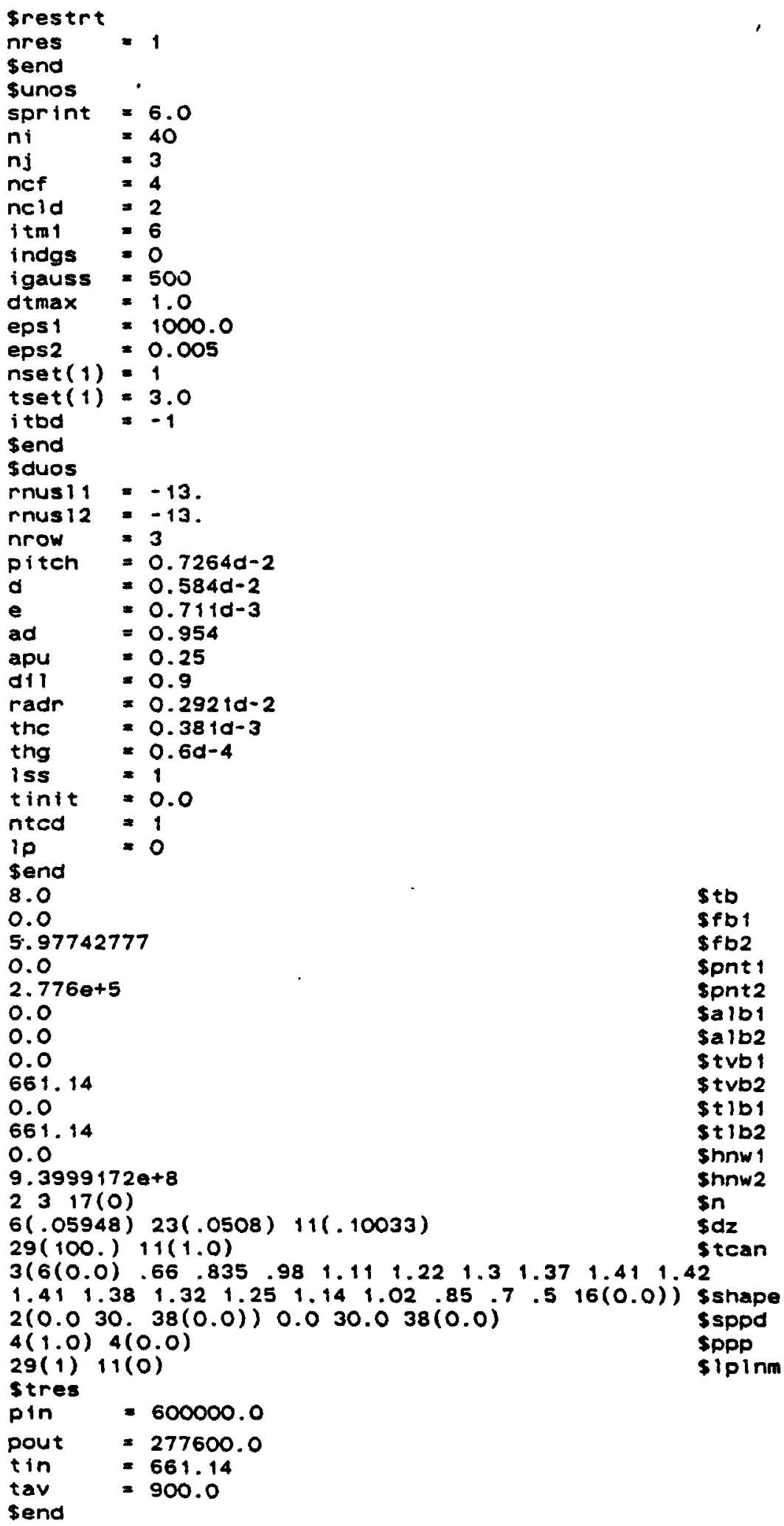


W-I SLSF BWT 2'

- Transient $(0.0-3.0 \mathrm{sec})$

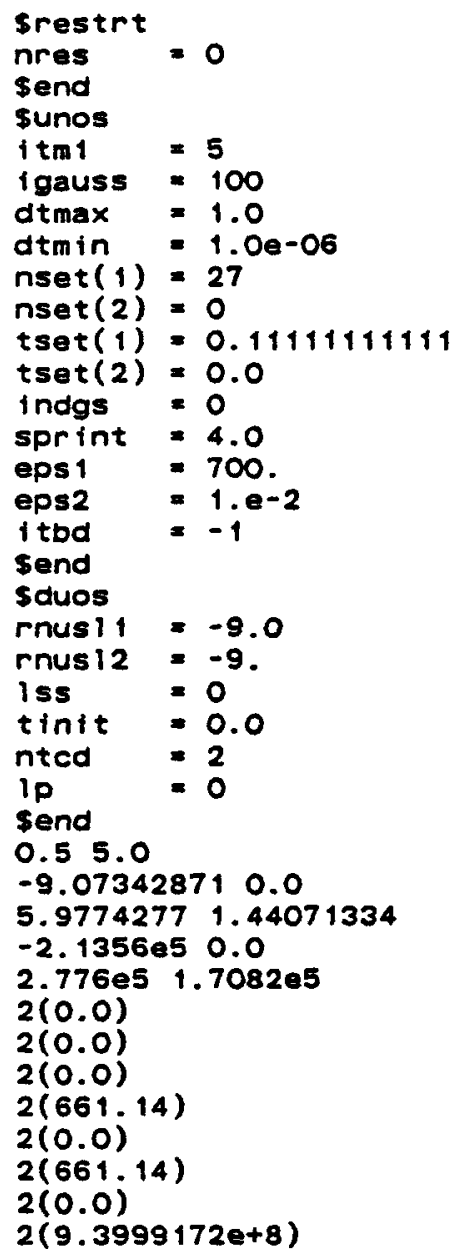

Stb

SVD 1

SVb2

Spnt 1 Spnt2

Salbl

$\$ a 1 b 2$

stvb 1

$\$+v b 2$

$\$+101$

Stib2

\$nnb1

Shnw2 
W-I SLSF BWT 2'

- Transient $(3.0-5.5 \mathrm{sec})$

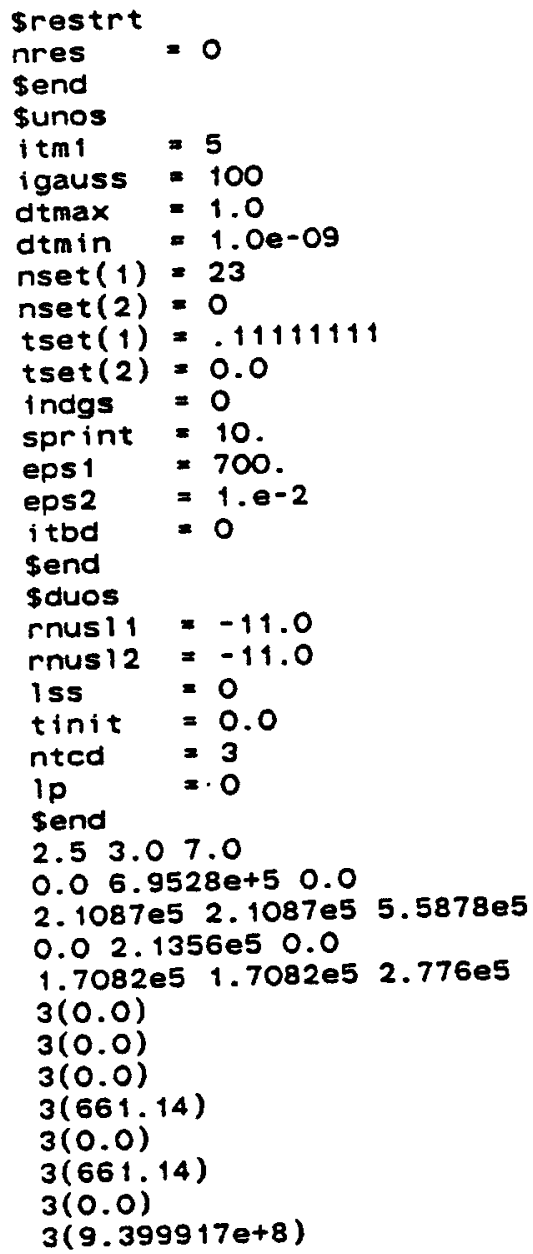

stb

Spnb 1

\$pnb2

Spnt 1

spnt2

Salbi

$\$$ alb2

stvb1

Stvb2

\$t 101

$\$$ t 1 b2

shnbl

Shnb2 
Appendix C.7: W-I SLSF BWT 7 $\mathrm{B}^{\prime}$

- Steady State

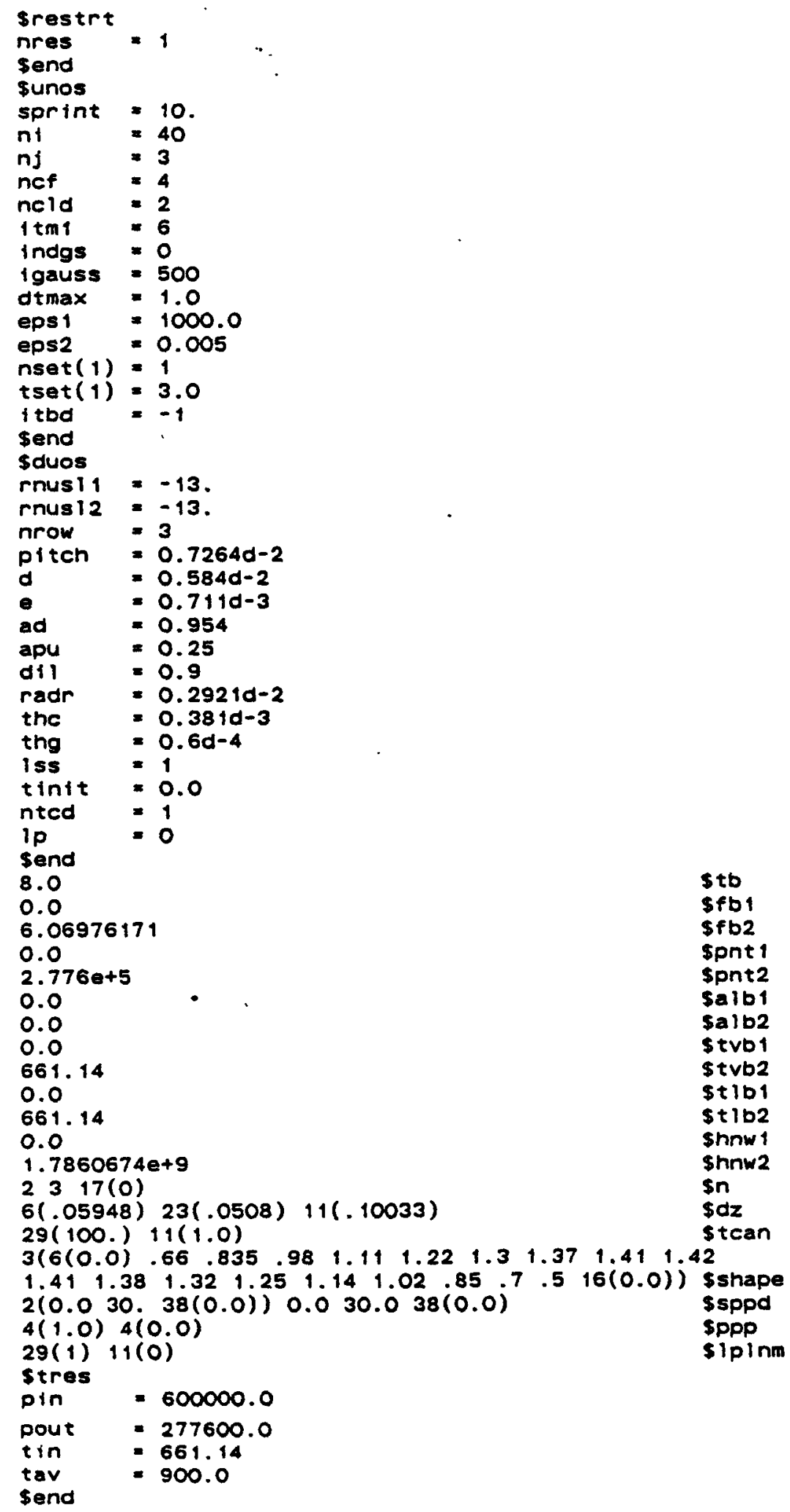


W-I SLSF BWT 7B'

- Transient $(0.0-1.5 \mathrm{sec})$

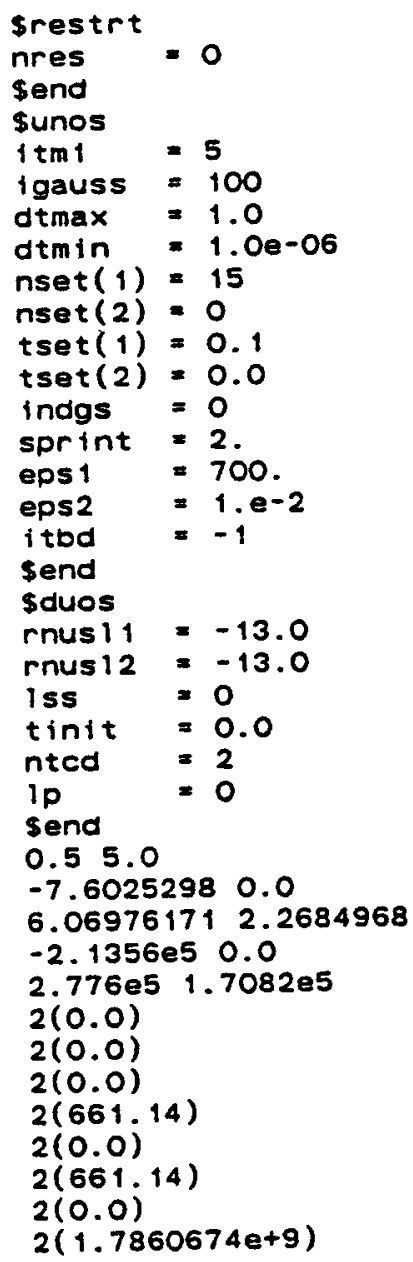

\$tb

Svb1

$\$ v b 2$

Spnt 1

Spnt2

Salb1

$\$ a 1 b 2$

\$tvb 1

$\$$ tvb2

Stibl

st 102

Shnb 1

snnw2 
W-I SLSF BWT 7B'

- Transient ( 1.5 - 5.0 sec)

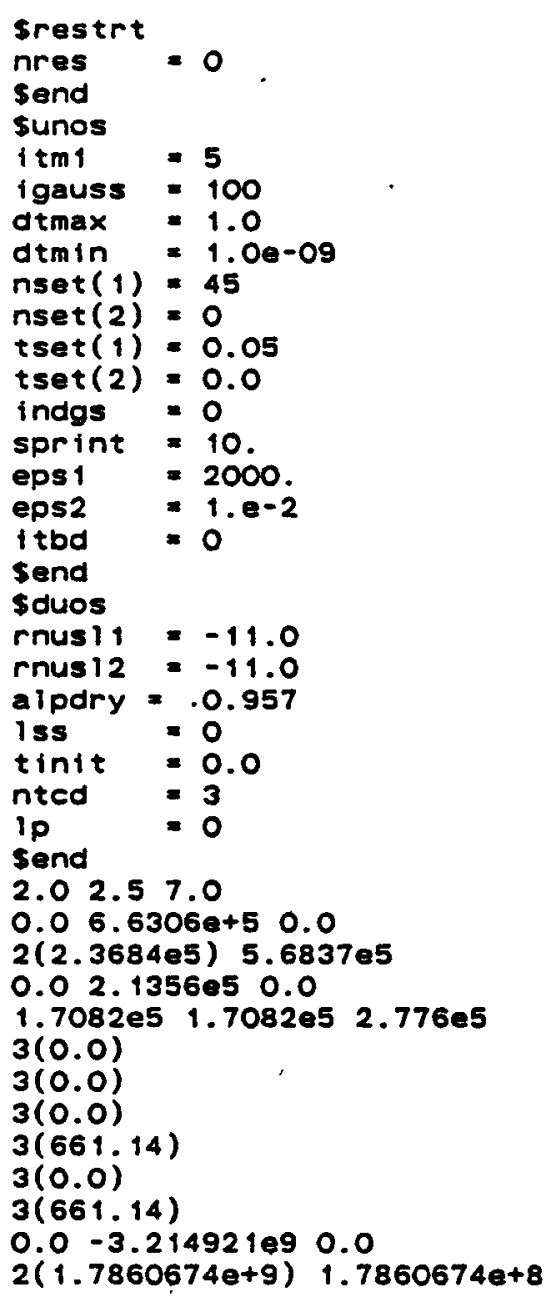

stb

Spnb 1 spnb2 Spnt 1 spnt2 \$alb1 $\$ a 1 b 2$ stvb 1 Stvb2 \$tibi st 162 Shnb 1 snnb2 
Appendix D

NATOF-2D HEXCAN MODEL

In NATOF-2D, the user specifies the heat capacity of the hexcan per unit area $\left(\mathrm{J} / \mathrm{m}^{2}{ }^{\circ} \mathrm{K}\right)$ for each axial level. Presently, this value is estimated by the user based on the properties of the can. However, this simple model has many limitations since it cannot take into account varying properties or dimensions of the hexcan, or heat losses to the environment.

The value chosen for the hexcan heat capacity has a pronounced effect of the temperature profile of the coolant. As an example, two cases were run with NATOF-2D for different values of the hexcan heat capacity. The simulation chosen for the test was BWT 2' (described in Chapter 6) and the input can be found in Appendix C.6. Table D.1 gives the liquid temperature at the end of the heated zone for the central channel at various points of time. The radial heat conduction nusselt numbers were $N u_{1}=13$, and $\mathrm{Nu}_{2}=13$. 


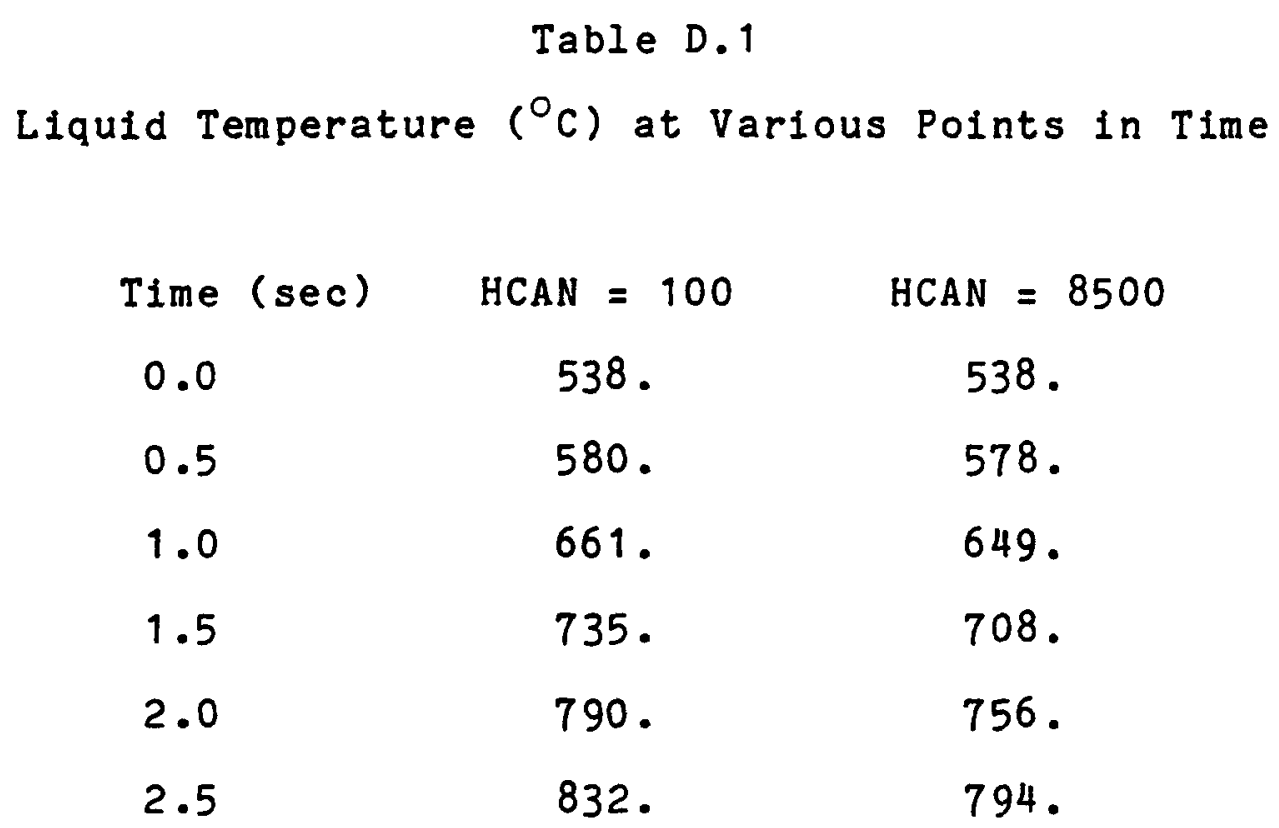

As can be seen, an inaccurate choice of the hexcan heat capacity can cause large liquid temperature differences. Thus, in two-phase transients, boiling inception time can be drastically altered. 
Appendix E

SPACER PRESSURE DROP MODEL

The spacer pressure drop feature of NATOF-2D allows the user to simulate pressure drops which occur in the bundle due to valve throttling, spacer wires, etc. The spacer pressure drop is calculated as follows:

$$
\Delta P=S P P D * \frac{\rho \cdot U^{2}}{2}
$$

where SPPD is specified for each cell.

Flow reversal occurs in NATOF-2D when the pressure in the first real cell exceeds the pressure at the boundary. Thus, by specifying a large boundary pressure, and a large value of SPPD, it is possible to prevent flow reversal during boiling transients.

As a sample test of this feature, simulations were run in which a constant flow of $2 \mathrm{~kg} / \mathrm{sec}$ was imposed on the bundle, with an outlet pressure of 2.5 bars. The geometric parameters used were from the W-1 SLSF Experiment. The value of SPPD for the bottom row of cells was varied over a wide range, and the inlet pressure necessary to maintain the flow rate was inferred. Table E. 1 gives values of SPPDs and the corresponding inlet pressure. 
Table E.1

Inlet Pressure vS. SPPD for Constant Flow Boundary Condition

$\begin{array}{rc}\text { SPPD } & \text { Inlet Pressure (bars) } \\ 0.0 & 5.16 \\ 10.0 & 5.26 \\ 100.0 & 6.12 \\ 500.0 & 9.96\end{array}$

As can be seen, it is possible to determine flow reversal time by the correct choice of the spacer pressure drop. Furthermore, if the experimental outlet and inlet pressure drops are accurately known, the spacer pressure drop feature can be used to obtain the correct inlet flow rate. 
flow map at time $=11.3415$ sec.

number of time steps $=\quad 161$

number of 1terations :

time step size $=0.26120-02$ sec.

cpu $\operatorname{time}=712.16$

inlet mass low rate $=0.5770330-01 \mathrm{~kg} / \mathrm{sec}$

outlet mass flow rate $=0.7576690+00 \mathrm{~kg} / \mathrm{sec}$

inlet enthalpy flow $-0.5035010+05$ watt outlet ollthalpy flow $0.9823160+06$ watt

channel number 1

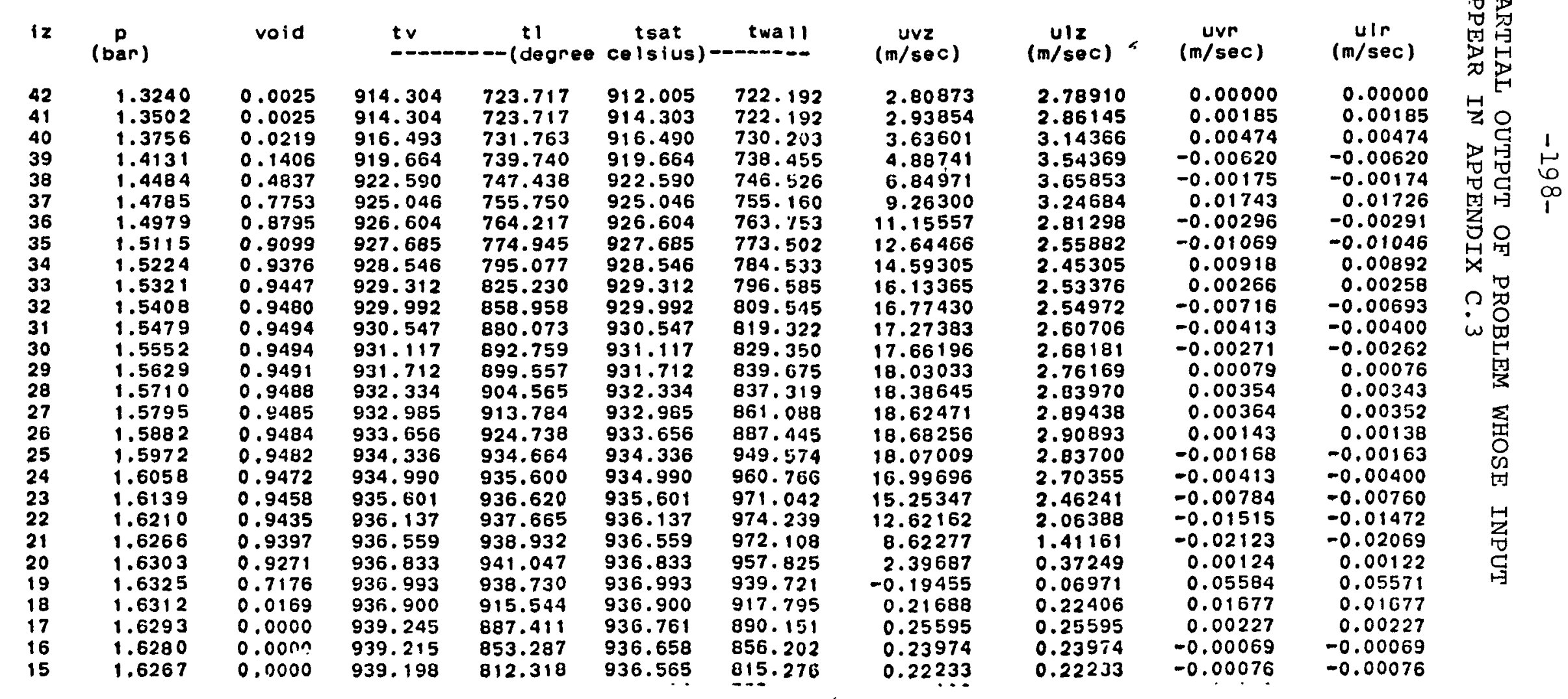




\begin{tabular}{|c|c|c|c|c|c|c|}
\hline $\begin{array}{l}16 \\
15 \\
14 \\
13 \\
12 \\
11 \\
10 \\
9 \\
6 \\
7 \\
6 \\
5 \\
4 \\
3 \\
2 \\
1\end{array}$ & $\begin{array}{l}1.6280 \\
1.6267 \\
1.6255 \\
1.6243 \\
1.6231 \\
1.6220 \\
1.6209 \\
1.6198 \\
1.6187 \\
1.6176 \\
1.6165 \\
1.6154 \\
1.6142 \\
1.6131 \\
1.6120 \\
1.6113\end{array}$ & $\begin{array}{l}0.00 n n \\
0.0000 \\
0.0000 \\
0.0000 \\
0.0000 \\
0.0000 \\
0.0000 \\
0.0000 \\
0.0000 \\
0.0000 \\
0.0000 \\
0.0000 \\
0.0000 \\
0.0000 \\
0.0000 \\
0.0000\end{array}$ & $\begin{array}{l}939.215 \\
939.198 \\
939.136 \\
939.004 \\
938.794 \\
938.507 \\
938.191 \\
937.718 \\
937.517 \\
935.479 \\
935.045 \\
935.666 \\
935.616 \\
935.541 \\
935.457 \\
387.770\end{array}$ & $\begin{array}{l}853.287 \\
812.318 \\
765.934 \\
716.213 \\
664.447 \\
611.886 \\
560.093 \\
509.717 \\
462.993 \\
416.119 \\
393.087 \\
390.952 \\
388.616 \\
387.967 \\
387.808 \\
387.770\end{array}$ & $\begin{array}{l}936.658 \\
936.565 \\
936.474 \\
936.384 \\
936.296 \\
936.210 \\
936.125 \\
936.043 \\
935.962 \\
935.880 \\
935.796 \\
935.712 \\
935.628 \\
935.543 \\
935.457 \\
935.406\end{array}$ & $\begin{array}{l}856.202 \\
815.270 \\
768.824 \\
718.979 \\
667.045 \\
614.262 \\
562.240 \\
511.548 \\
464.089 \\
416.466 \\
398.212 \\
390.990 \\
388.626 \\
387.969 \\
387.008 \\
387.808\end{array}$ \\
\hline
\end{tabular}

$\begin{array}{ll}0.23974 & 0.23974 \\ 0.22233 & 0.22233 \\ 0.21106 & 0.21106 \\ 0.20355 & 0.20355 \\ 0.19773 & 0.19773 \\ 0.19274 & 0.19274 \\ 0.18825 & 0.18825 \\ 0.18441 & 0.18441 \\ 0.18099 & 0.18099 \\ 0.18042 & 0.18042 \\ 0.18100 & 0.18100 \\ 0.18249 & 0.18249 \\ 0.18417 & 0.18417 \\ 0.18467 & 0.18467 \\ 0.18292 & 0.18292 \\ 0.18292 & 0.18292\end{array}$
$-0.00069$
$-0.00076$
$-0.00039$
$-0.00016$
$-0.00007$
$-0.00005$
$-0.00004$
$-0.00003$
$-0.00002$
0.00001
0.00005
0.00009
$0.00010^{\circ}$
0.00003
$-0.00010$
0.00000

$-0.00069$

$-0.00076$

$-0.00039$

$-0.00016$

$-0.00007$

$-0.00005$

$-0.00004$

$-0.00003$

$-0.00002$

0.00001

0.00005

0.00009

0.00010

0.00003

$-0.00010$

0.00000 (bar)

1.3240

1.3502

1.4133

1.4484

1.4778

1.4980

1.5119

1.5220

1.5320

1.5410

1.5480

1.5553

1.5629

1.5709

1.5793

1.5832

1.5972

1.6059

1.6141

1.6215 vold

0.0032

.0032

0.0246

0.1954

0.5861

0.8179

0.8626

0.9151

0.9400

0.9403

0.9494

0.9498

0.9505

0.9502

.9492

0.9484

0.9479

0.9479

$0.946=$

.9452

0.9421

channel number

tv

$t$

(degr

914. 297

914.297

916.472

919.684

922.596

924.992

926.613

927.716

928.520

929.305

930.011

930.557

931.124

931.710

932.325

932.976

933.653

934.340

935.000

935.620

936.172
724.332

724.332

733.267

742.515

751.942

762.102

771.577

00652

826.719

864.631

882.380

894.599

902.509

907.412

915.767

925.415

934.675

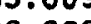

936.669

937.913 tsat

twa I!

$912.005 \quad 722.680$

722.680

$916.469 \quad 731.532$

919.684741 .160

922.596751 .028

$924.992 \quad 761.498$

$926.613 \quad 770.883$

$927.716 \quad 781.281$

$928.520 \quad 792.532$

929.305805 .750

$930.011 \quad 819.832$

$930.557 \quad 829.535$

$931.124 \quad 839.339$

$931.710 \quad 848.931$

932.326844 .384

$932.977 \quad 866.811$

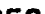

35.000

936.172
891.275

948.597

958.983

968.288

970.950 uvz

$(m / s \theta c)$

2.76187

2.89537

3.70797

5.23751

7.86026

10.16014

10.84887

12.66733

14.66586

15.96186

16.77386

17.34419

17.78258

18.14940

18.45993

18.64648

18.67160

18.05545

17.01460

15.26988

12.35471 $u 12$
$(m / s e c)$

2.73827

2.79878

3.08188

3.54216

3.73747

3.32169

2.80917

2.49359

2.48910

2.57973

2.53586

2.59818

2.66169

2.74732

2.84136

2.90881

2.92745

2.85070

2.71860

2.47962

2.03568 uvr
$(\mathrm{m} / \mathrm{sec})$

0.00000

0.00251

$-0.00313$

$-0.00152$

0.03048

0.02046

$-0.06563$

0.00142

0.02238

$-0.00466$

0,00442

0.00128

0.00143

0.00406

0.00571

0.00460

0.00092

$-0.00240$

0.0055

-0.01208
-0.02705

$-0.00313$

-0.00152
0.03039

0.02028

$-0.06462$

0.00138

0.02176

-0.00452
0.00427

0.00124

0.00139

0.00392

0.00553

0.00445

0.00089

$-0.00232$

$-0.00537$

$-0.01173$

$-0.02630$ 


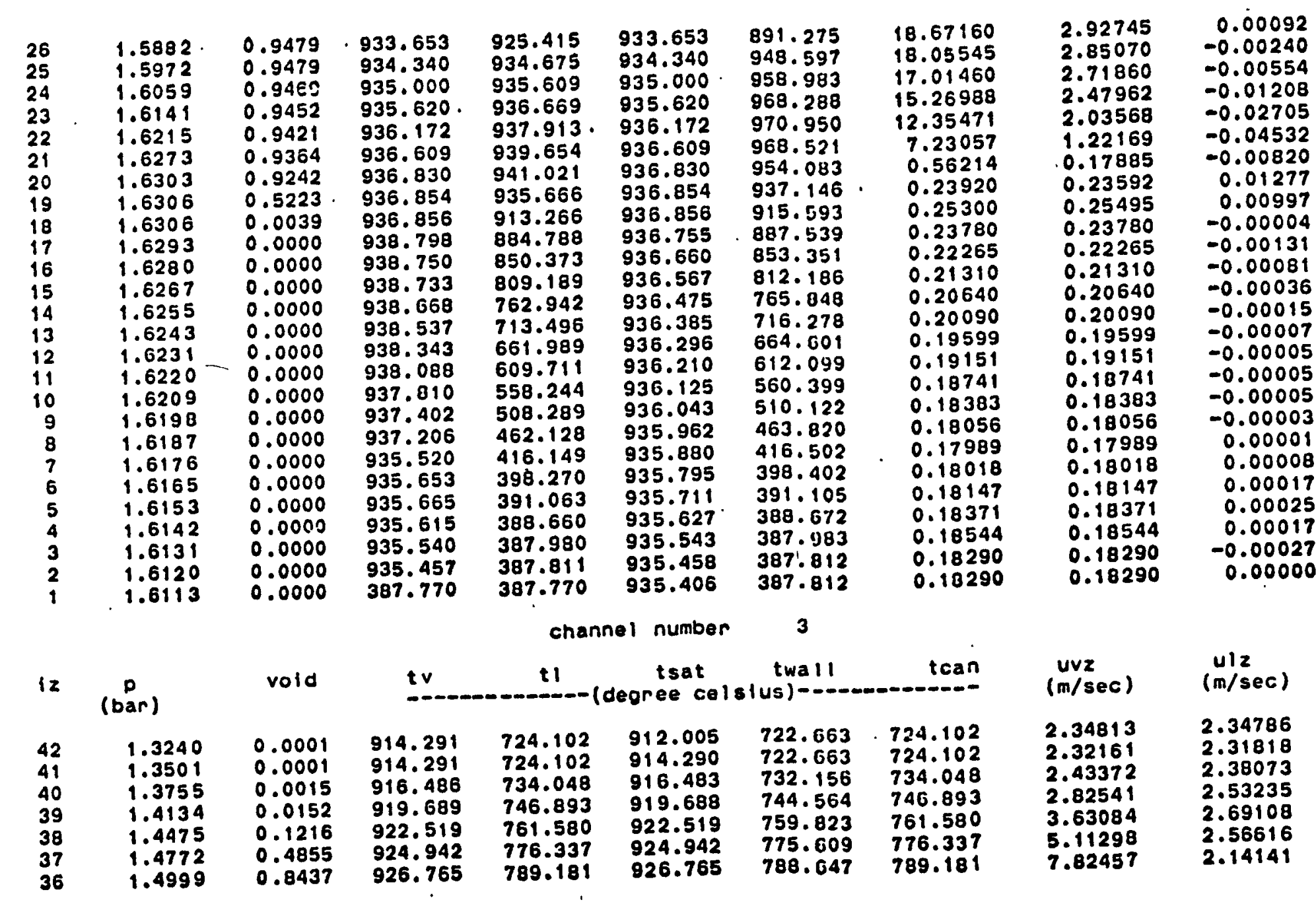




\begin{tabular}{|c|c|c|c|c|c|c|c|c|c|}
\hline $\begin{array}{l}35 \\
34 \\
33 \\
32 \\
31 \\
30 \\
29 \\
28 \\
27 \\
26 \\
25 \\
24 \\
23 \\
22 \\
21 \\
20 \\
19 \\
18 \\
17 \\
16 \\
15 \\
14 \\
13 \\
12 \\
11 \\
10 \\
9 \\
8 \\
7 \\
6 \\
5 \\
4 \\
3 \\
2 \\
1\end{array}$ & $\begin{array}{l}1.5118 \\
1.5214 \\
1.5321 \\
1.5409 \\
1.5480 \\
1.5553 \\
1.5628 \\
1.5707 \\
1.5792 \\
1.5882 \\
1.5973 \\
1.6061 \\
1.6144 \\
1.6221 \\
1.6284 \\
1.6305 \\
1.6303 \\
1.6303 \\
1.6293 \\
1.6280 \\
1.6268 \\
1.6255 \\
1.6243 \\
1.6231 \\
1.6220 \\
1.6209 \\
1.6198 \\
1.6187 \\
1.6176 \\
1.6165 \\
1.6153 \\
1.6142 \\
1.6131 \\
1.6120 \\
1.6113\end{array}$ & $\begin{array}{l}0.9269 \\
0.9180 \\
0.9375 \\
0.9439 \\
0.9453 \\
0.9472 \\
0.9468 \\
0.9458 \\
0.9447 \\
0.9443 \\
0.9447 \\
0.9442 \\
0.9412 \\
0.9333 \\
0.9067 \\
0.7020 \\
0.2680 \\
0.0019 \\
0.0000 \\
0.0000 \\
0.0000 \\
0.0000 \\
0.0000 \\
0.0000 \\
0.0000 \\
0.0000 \\
0.0000 \\
0.0000 \\
0.0000 \\
0.0000 \\
0.0000 \\
0.0000 \\
0.0000 \\
0.0000 \\
0.0000\end{array}$ & $\begin{array}{l}927.713 \\
928.472 \\
929.315 \\
930.002 \\
930.555 \\
931.121 \\
931.702 \\
932.314 \\
932.968 \\
933.651 \\
934.344 \\
935.010 \\
935.642 \\
936.222 \\
936.693 \\
936.847 \\
936.831 \\
936.836 \\
937.786 \\
937.716 \\
937.595 \\
937.452 \\
937.308 \\
937.153 \\
936.976 \\
936.790 \\
936.545 \\
936.377 \\
935.680 \\
935.700 \\
935.675 \\
935.615 \\
935.539 \\
935.458 \\
387.770\end{array}$ & $\begin{array}{l}806.221 \\
817.640 \\
841.307 \\
868.943 \\
881.914 \\
894.130 \\
899.128 \\
899.633 \\
909.964 \\
921.601 \\
934.564 \\
935.556 \\
936.799 \\
938.643 \\
941.081 \\
937.777 \\
924.139 \\
904.095 \\
874.505 \\
838.230 \\
797.743 \\
753.030 \\
704.672 \\
654.042 \\
602.703 \\
552.252 \\
503.598 \\
458.816 \\
415.774 \\
398.497 \\
391.241 \\
388.736 \\
388.004 \\
387.816 \\
387.770\end{array}$ & $\begin{array}{l}927.712 \\
928.472 \\
929.315 \\
930.002 \\
930.555 \\
931.121 \\
931.702 \\
932.314 \\
932.968 \\
933.651 \\
934.345 \\
935.010 \\
935.642 \\
936.222 \\
936.693 \\
936.847 \\
936.831 \\
936.836 \\
936.755 \\
936.662 \\
936.568 \\
936.476 \\
936.385 \\
936.297 \\
936.210 \\
936.126 \\
936.043 \\
935.962 \\
935.880 \\
935.795 \\
935.711 \\
935.626 \\
935.542 \\
935.459 \\
935.406\end{array}$ & $\begin{array}{l}801.344 \\
814.924 \\
829.134 \\
843.314 \\
853.189 \\
861.994 \\
870.457 \\
862.147 \\
880.900 \\
899.784 \\
943.994 \\
953.065 \\
960.568 \\
959.247 \\
946.747 \\
939.050 \\
925.918 \\
906.129 \\
876.035 \\
840.926 \\
800.003 \\
755.304 \\
706.882 \\
656.122 \\
604.603 \\
553.963 \\
505.048 \\
460.152 \\
416.088 \\
398.636 \\
391.291 \\
388.751 \\
388.008 \\
387.817 \\
387.817\end{array}$ & $\begin{array}{l}806.221 \\
817.640 \\
841.307 \\
868.943 \\
881.914 \\
894.130 \\
899.128 \\
878.998 \\
896.462 \\
913.215 \\
930.023 \\
932.410 \\
933.121 \\
934.261 \\
940.342 \\
937.468 \\
923.782 \\
903.736 \\
874.105 \\
837.852 \\
797.409 \\
752.745 \\
704.431 \\
653.842 \\
602.545 \\
552.133 \\
503.527 \\
458.768 \\
415.774 \\
398.497 \\
391.241 \\
388.736 \\
388.004 \\
387.816 \\
387.816\end{array}$ & $\begin{array}{r}9.66715 \\
10.81656 \\
12.20306 \\
12.85329 \\
13.38528 \\
13.80544 \\
14.14821 \\
14.47696 \\
14.68455 \\
14.76841 \\
14.38129 \\
13.55669 \\
11.89976 \\
8.51273 \\
2.10520 \\
0.22167 \\
0.28323 \\
0.18297 \\
0.18005 \\
0.18869 \\
0.19229 \\
0.19148 \\
0.18871 \\
0.18541 \\
0.18221 \\
0.17933 \\
0.17694 \\
0.17464 \\
0.17395 \\
0.17292 \\
0.17110 \\
0.16851 \\
0.16678 \\
0.16960 \\
0.16960\end{array}$ & $\begin{array}{l}1.99827 \\
2.13968 \\
2.12147 \\
2.11556 \\
2.15590 \\
2.19861 \\
2.27296 \\
2.36172 \\
2.43020 \\
2.45266 \\
2.39341 \\
2.29605 \\
2.11027 \\
1.67252 \\
0.65164 \\
0.29299 \\
0.27942 \\
0.18343 \\
0.18005 \\
0.18869 \\
0.19229 \\
0.19148 \\
0.18871 \\
0.18541 \\
0.18221 \\
0.17933 \\
0.17694 \\
0.17464 \\
0.17395 \\
0.17292 \\
0.17110 \\
0.16851 \\
0.16678 \\
0.16960 \\
0.16960\end{array}$ \\
\hline
\end{tabular}




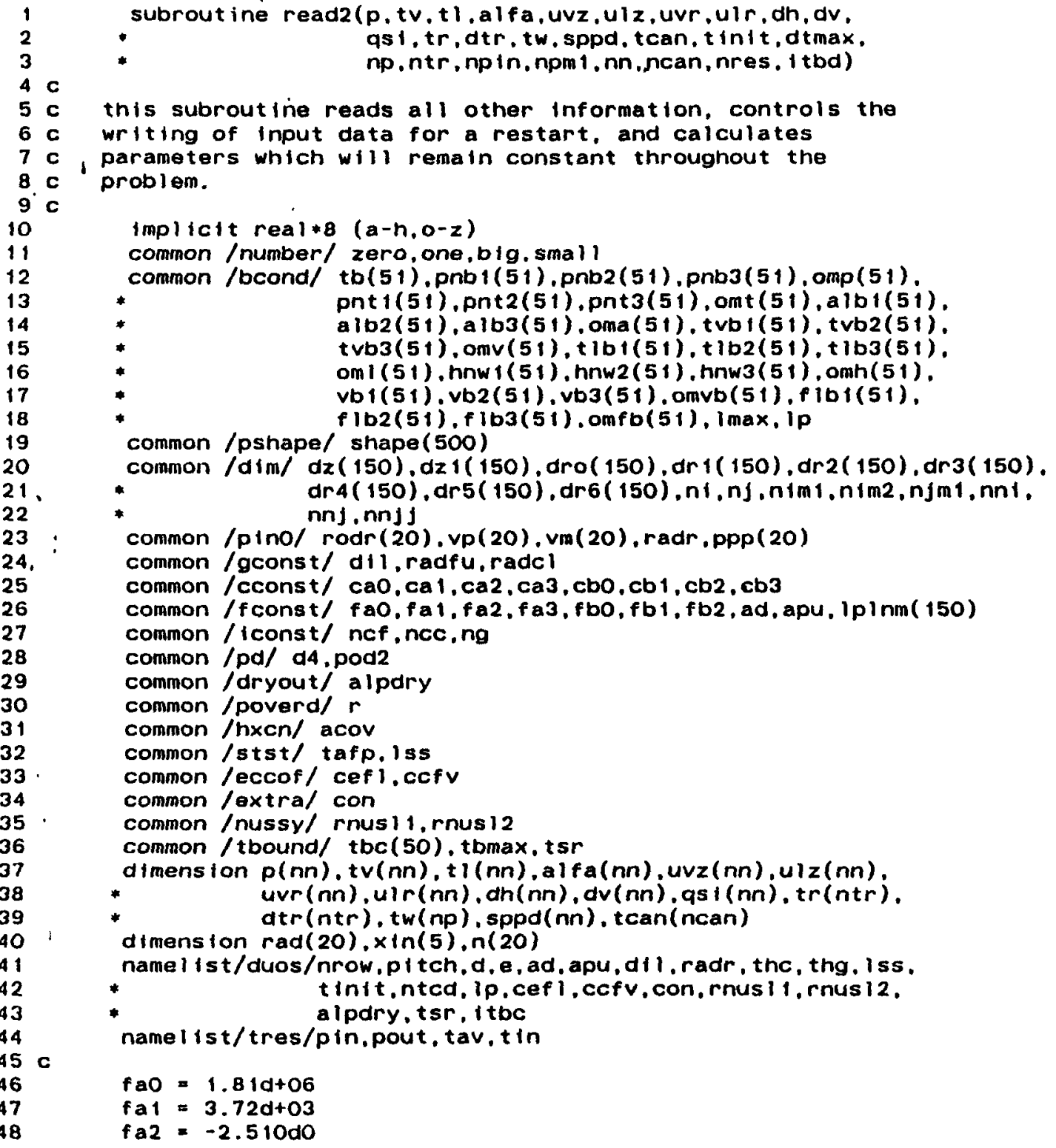




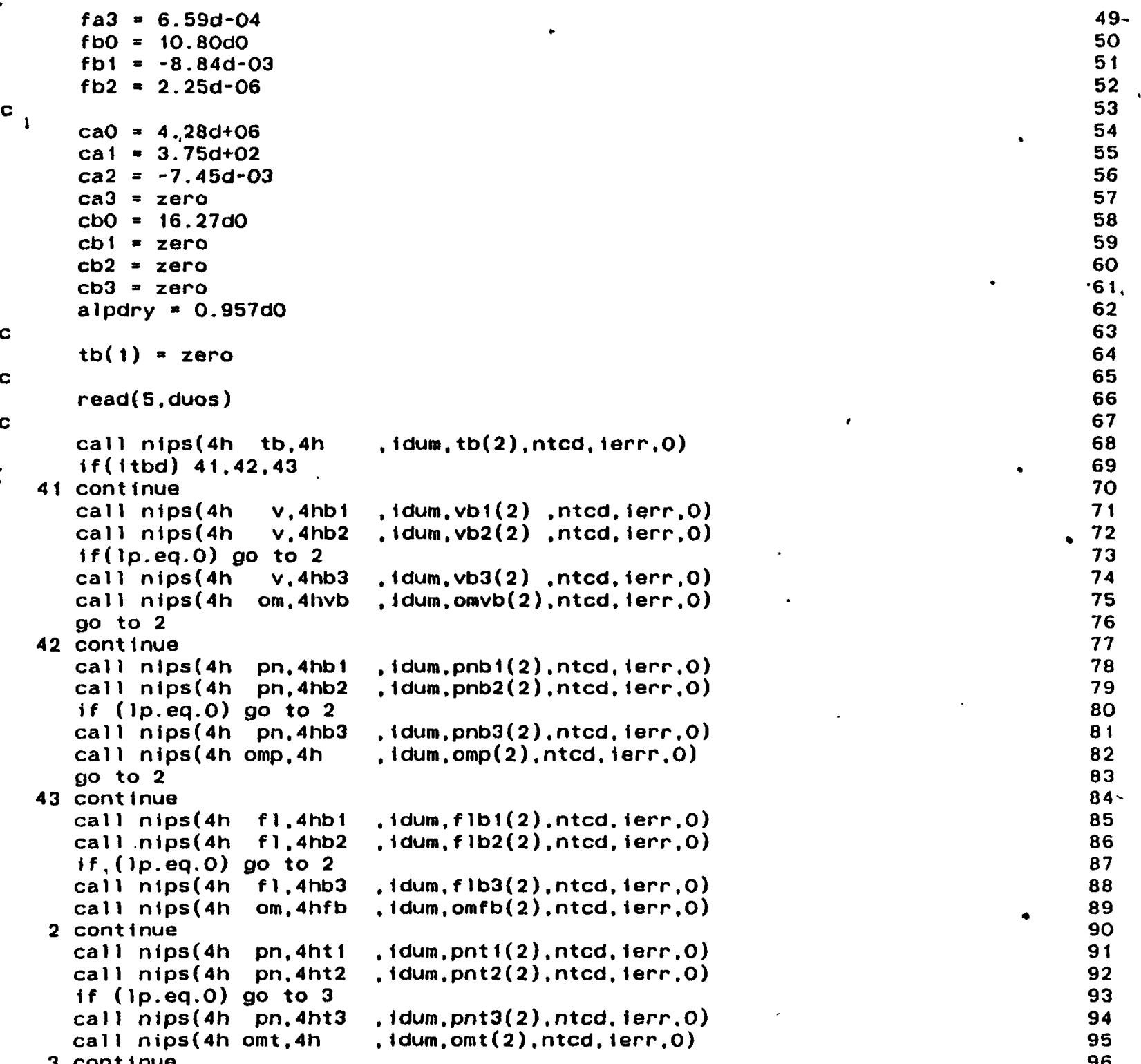

49.

50 


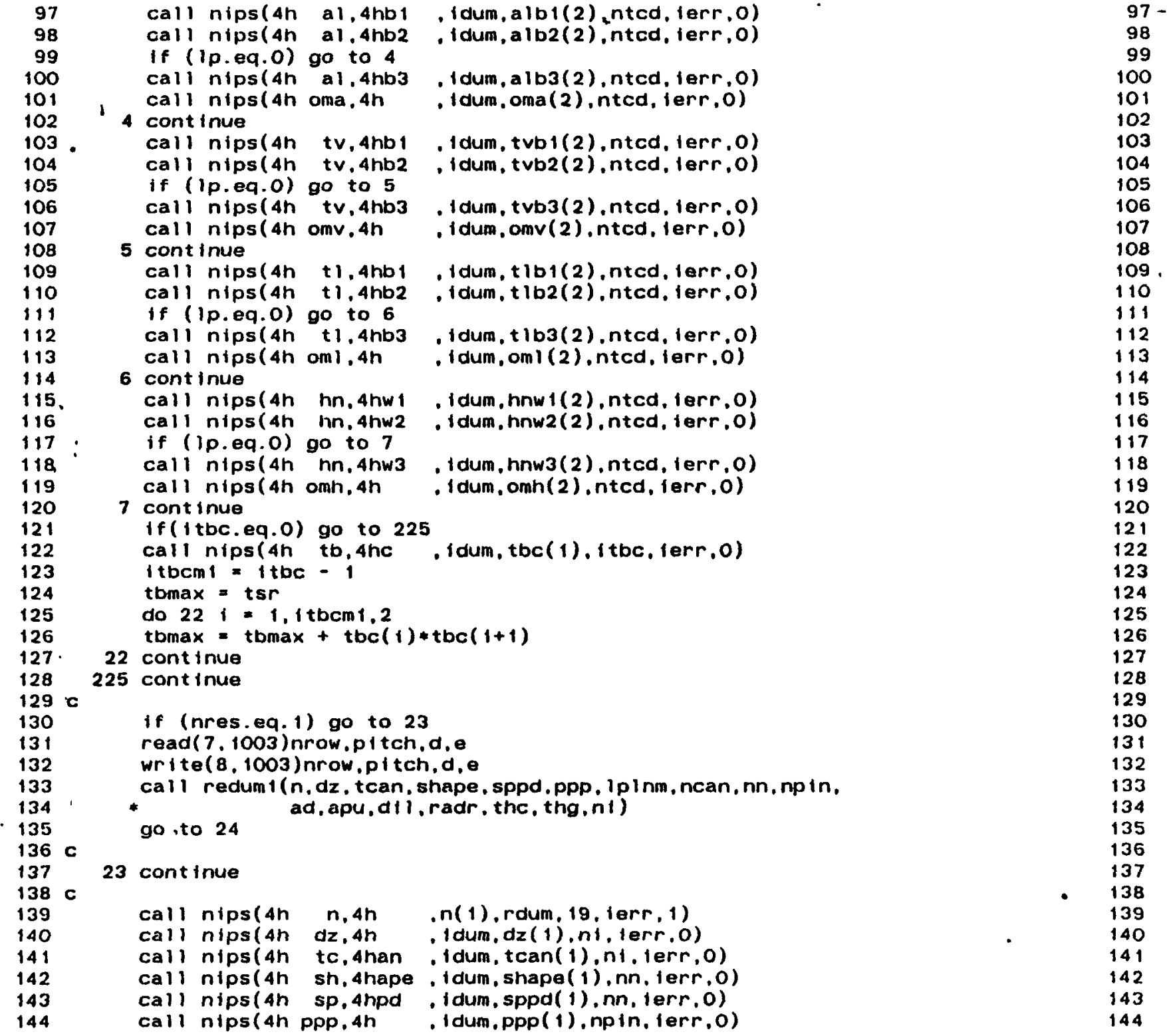


call nips(4h $1 \mathrm{pl}, 4 \mathrm{hnm}, 1 \mathrm{plnm}(1)$, rdup, $n 1,1 \mathrm{err}, 1)$

$1 \max =n t c d+2$

do $25 \mathrm{ko}=1, \mathrm{nn}$

$q$ si $(k o)=(4 . * d /(p i t c h-d)) * * 2$

25 continue

povd $=p i t c h / d$

pod2 $=$ povd $*$ povd

$d 4=4 . / d$

$r=-16.15+24.96 *$ povd $-8.55 *$ povd*povd

$d z 1(1)=d z(1)$

do $111 i=2, n i$

111 cont inue

$=(d z(1)+d z(1-1)) / 2.0$

c

a1 $=\operatorname{dsart}(3.0 \mathrm{do}) / 2.0$

$a_{2}=3.1415927 / 4.0$

$w=p i t c h-d$

$x=(p i t c h * p \mid t c h * a 1-(d * d+w * w) * a 2) / a 2 / d$.

$x i=4.0 / x$

$x i x=x / a 1 / p \mid t c h * 6$.

do $8 j=1, \mathrm{nJmt}$

do $81=1 . n 1$

ko $=(1-1) * n 1+t$

$\operatorname{dh}(k o)=x$

8 continue

$\operatorname{dr} 5(1)=x i x *(n(1)-1) * d * a 2$

do $9 j=2, n j m 1$

$n 41=n(j)-1$

$n 42=n(j-1)-1$

dn4 $=n 41 * n 41-n 42 * n 42$

$\operatorname{dr} 4(j)=\operatorname{dn} 4 * x * a 2 * d * 3.0$

$\operatorname{dr} 5(j)=x i x * d * a 2 * n 41$

$\operatorname{dr} 6(j)=\operatorname{dr} 4(j) / 1.5 /(n 41+n 42) / w$

$n x=n(j)-n(j-1)$

$n \times 1=2 * n 41$

$n \times 2=(2 * n 42+n x) * n x$

$\mathrm{dn} \times 1=n \times 1$

$\operatorname{dr} 1(j)=a n \times 1 / n \times 2 / p 1 t c h / a_{1}$ 


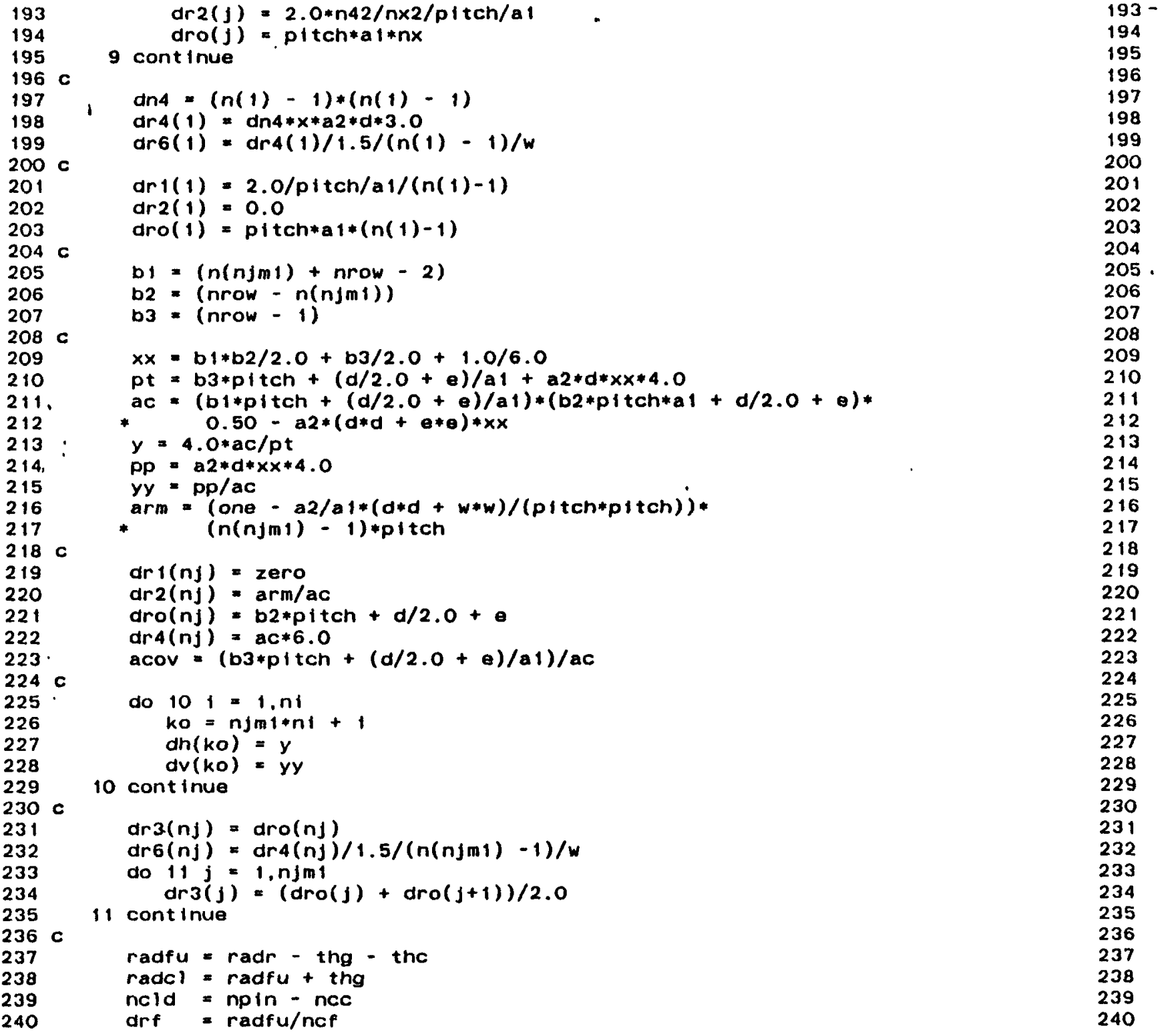




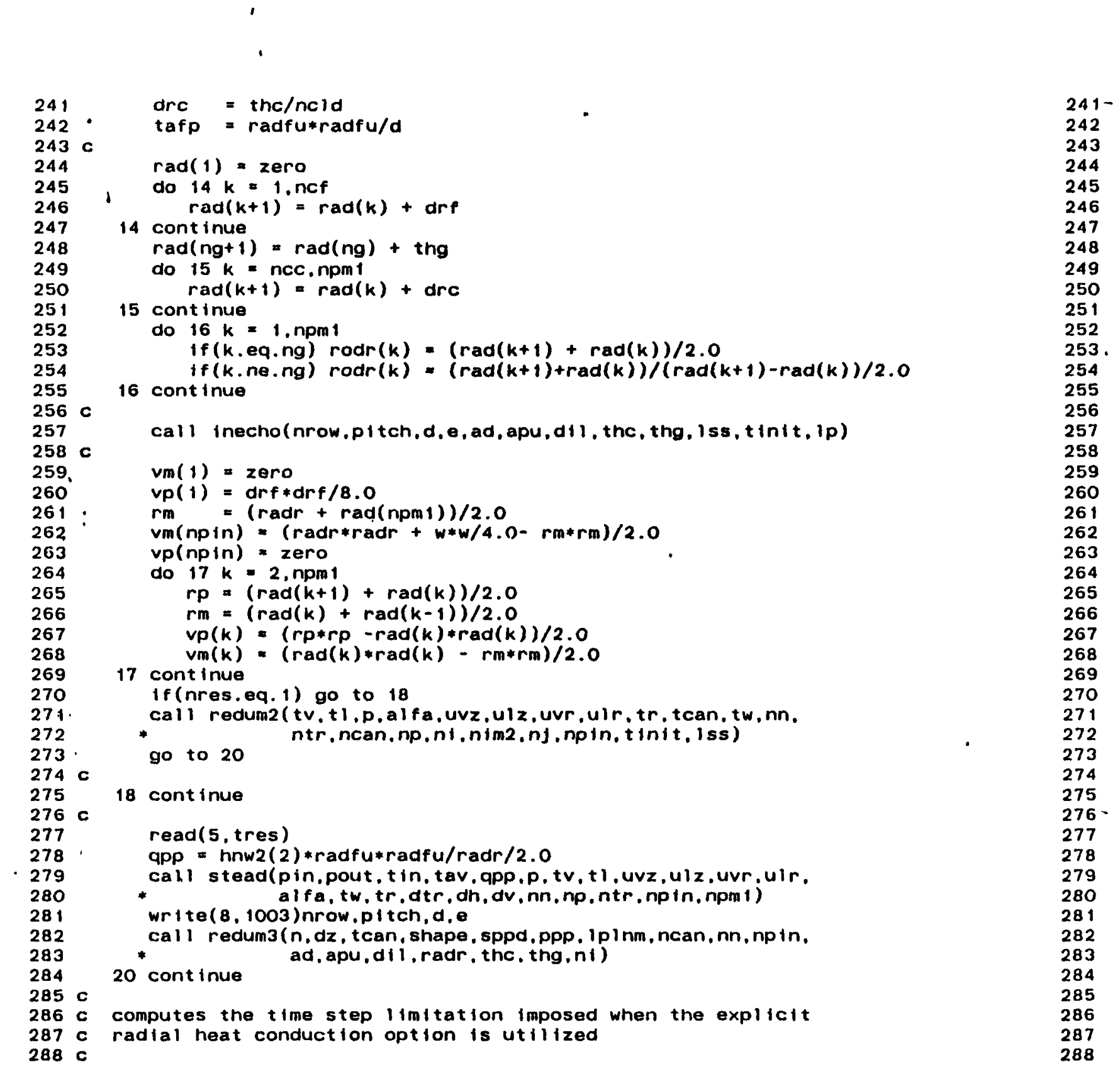


If (rnusil. le.zero) return

289$\operatorname{drmin}=\operatorname{dr} 6(1)$ do $37 t=2 . n j$

37

drmin a dmint(drmin, dr6(i))

290

291

292

$\operatorname{drmin}=d r m i n * d r m i n / d m a x 1($ rnus 11, rnus 12$) * 1.8623 d+03$ dtmax $=d m i n 1(d t \max , d r m i n)$

$$
\text { return }
$$

2961003 format $(15.3 d 15.9)$

format $(15,3 d 15.9)$ 


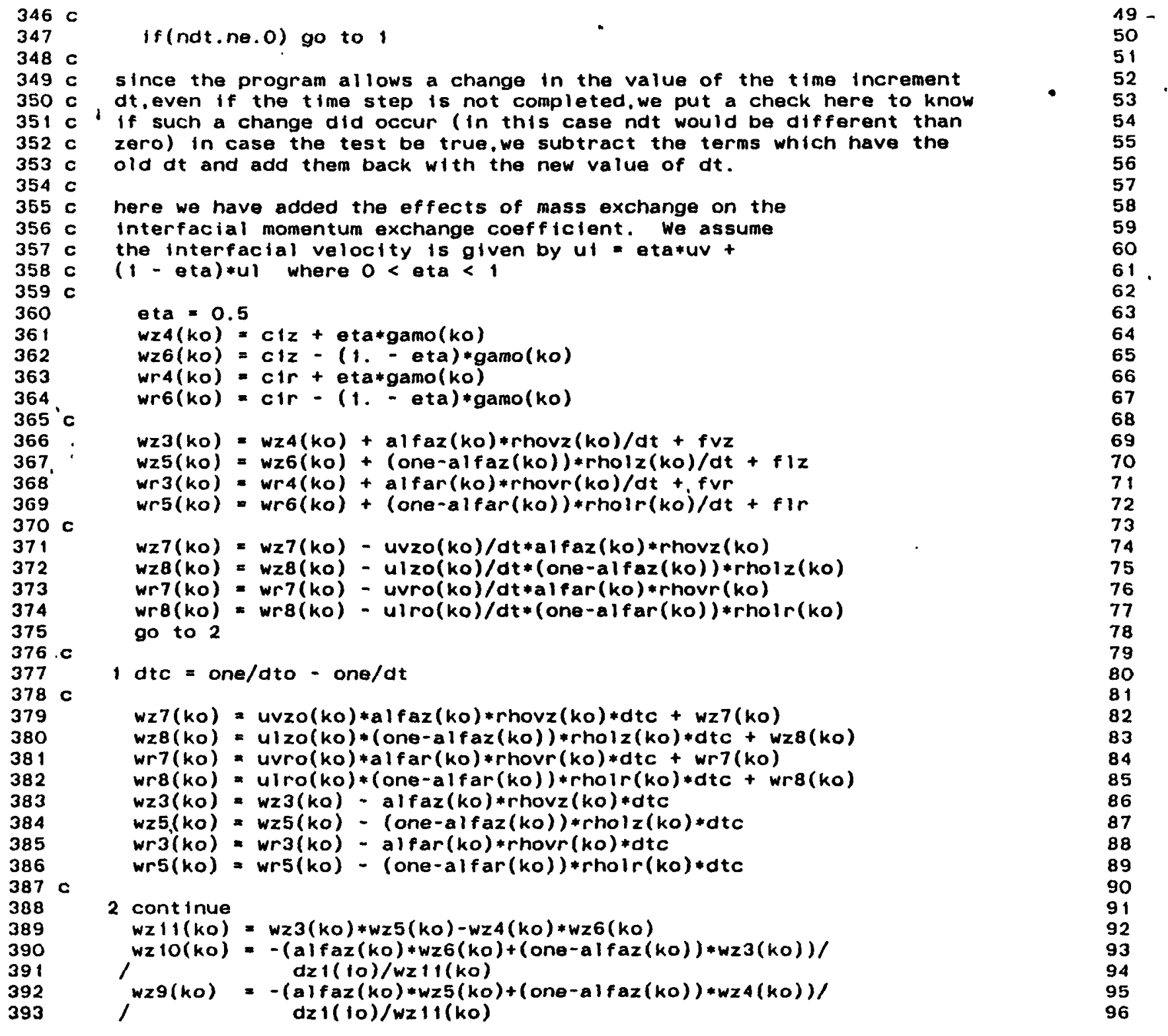


these terms are only used for the plow boundary condition they are the explicit terms of the non-discretized Iiquid and vapor momentum equations

do 5 i $1 . n j$

ko $=(i-1) * n i+.2$

$\exp \lim (1)=-(w z 7(k 0) * w z 6(k 0)+w z 3(k 0) * w z 8(k 0)) / w z 11(k 0)$

$\operatorname{expvm}(i)=-(w z 7(k 0) * w z 5(k 0)+w z 4(k 0) * w z 8(k 0)) / w z 11(k 0)$

5 cont inue

return

end 


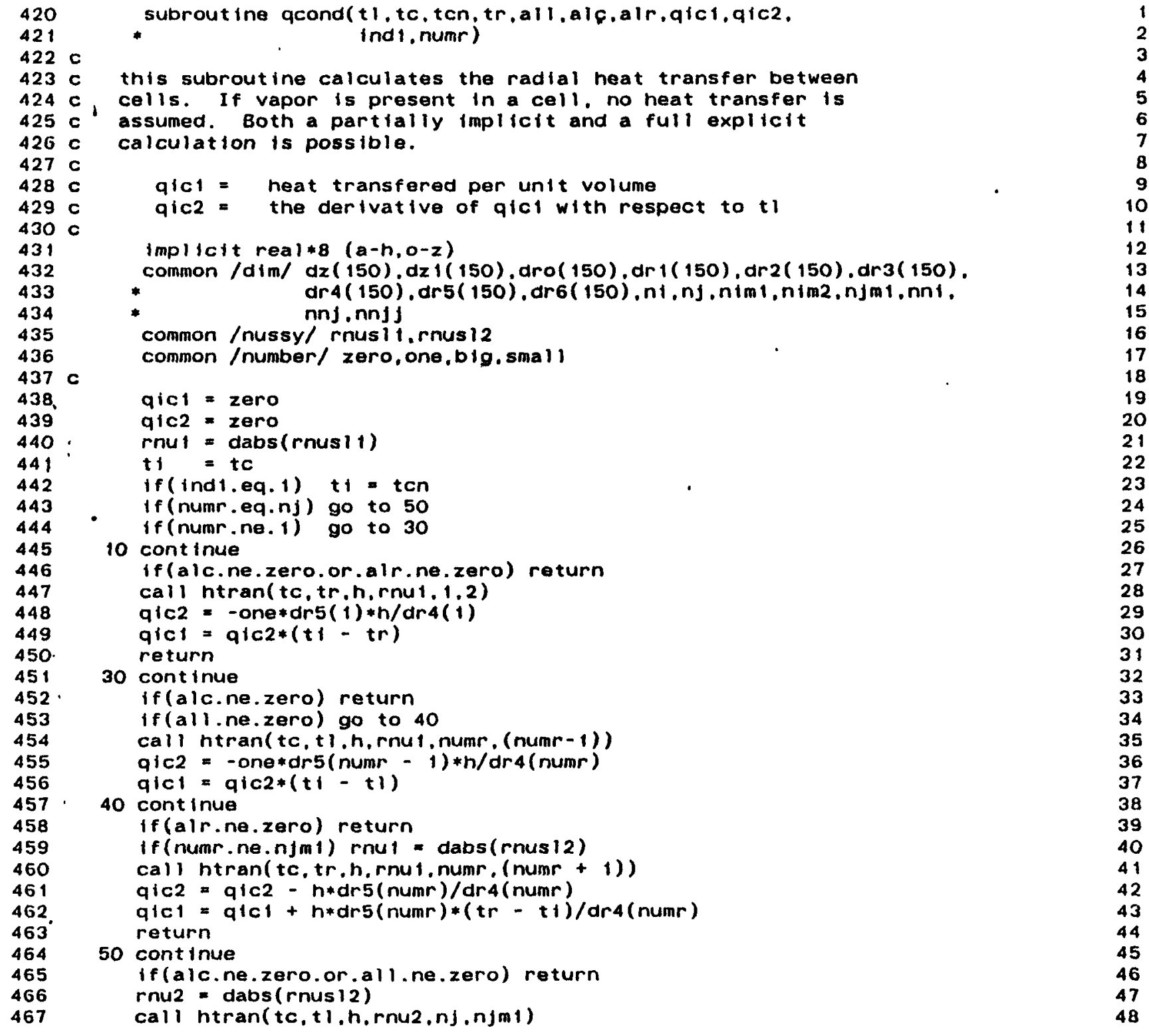


468

468

469

470
471

$q\{c 2=-o n \theta * d r 5(n j m 1) * n / d r 4(n j)$

qict $=q 102 *(t)-t 1)$

return

end

$49-$

50

51

1 
472 subroutine ntran(t1,t2,h,rnu, nut.nu2)

$473 \mathrm{C}$

$474 \mathrm{c}$ this subroutine calculates the intercell heat transfer coefficient

$475 \mathrm{c}$ for subrout ine acond

476 C implicit real*o $(a-n, 0-z)$

479

480

$481 \mathrm{C}$

482

483

$484 \mathrm{C}$

485

486

comnion /dim/ dz(150),dzl(150), dro( 150), dr1 (150), dr2(150), dr3 (150)

* $\operatorname{dr4}(150), \operatorname{dr} 5(150), \operatorname{dr6}(150), n 1, n\}, n i m 1, n i m 2, n j m 1, n n 1$,

conv1 $=\operatorname{cond} 1(t 1) / d r 6(n u 1)$

conv2 $=$ condi $($ t2)/dr6(nu2)

$n=2 . * r n u * \operatorname{conv} 1 * \operatorname{conv} 2 /(\operatorname{conv} 1+\operatorname{conv} 2)$

return

end 


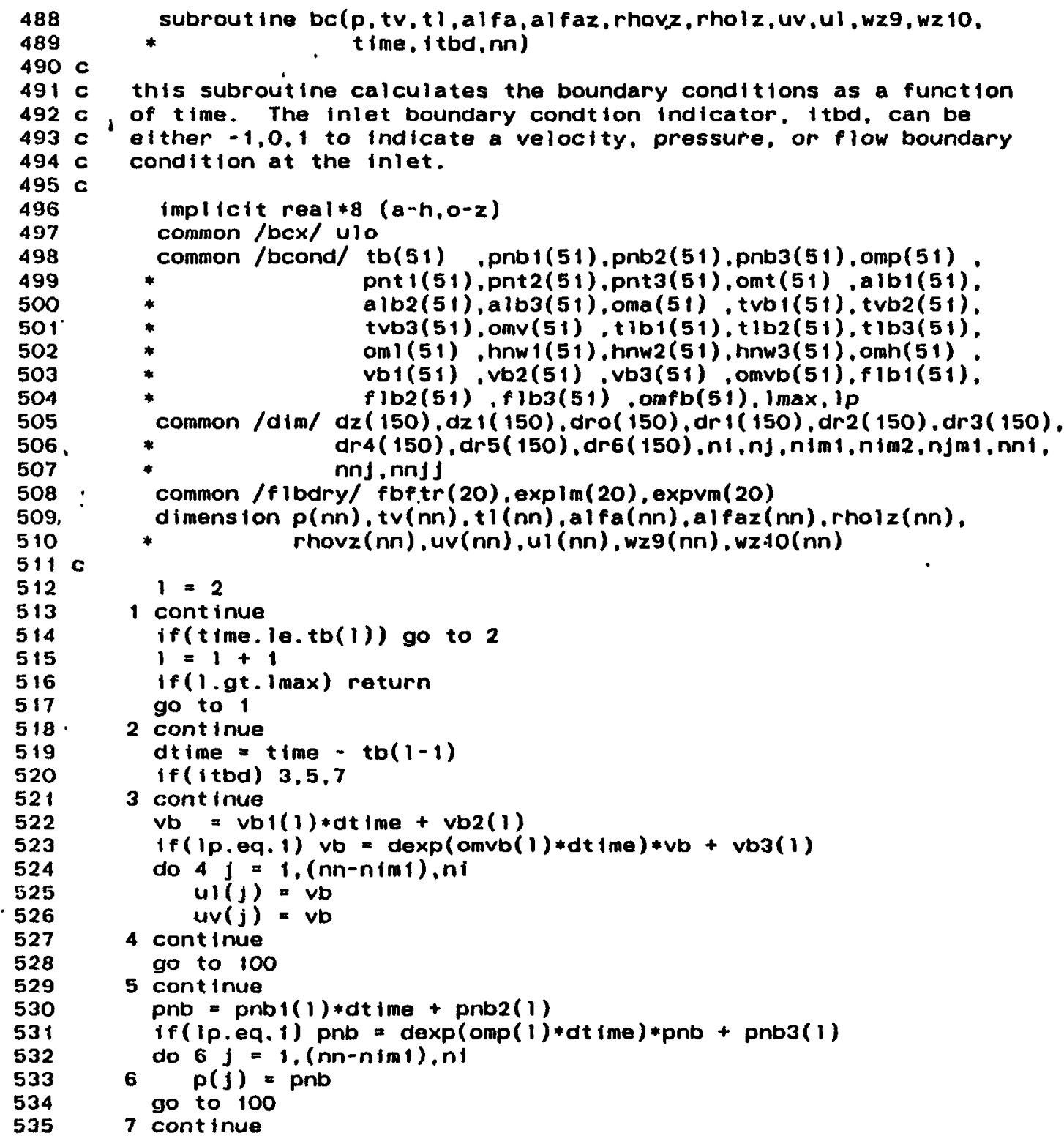

this subrout ine calculates the boundary conditions as a function

of time. The inlet boundary condtion indicator. itbd, can be

either $-1,0,1$ to indicate a velocity. pressure, or flow boundary condition at the inlet.

implicit real*8 $(a-h, o-z)$

common /bcx/ ulo

common /bcond/ tb(51) ,pnb1(51).pnb2(51).pnb3(51). omp (51) $*$

*

alb2(51), alb3(51), oma(51), tvb1(51), tvb2(51)

trb3(51), omv(51), tib1(51),t1b2(51),t tb3(51)

om1 (51). hnw1(51).hnw2(51).hnw3(51). Omh(51)

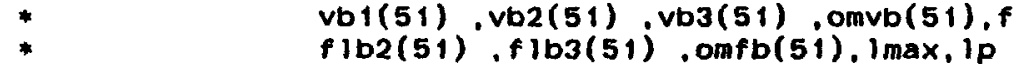

common /dim/ dz (150),dzi(150),dro(i50),dri(i50),dr2(150),dr3(150).

* dr4 (150) dr5(150) dr6(150), ni,nj, nim1, nim2, njm1, nni,

nnj, innjj

/fibdry/ fbftr(20), explm(20), expvm(20)

dimension $p(n n)$, tv(nn), tl (nn), al fa(nn), alfaz $(n n)$, rholz $(n n)$, * rhovz $(n n), u v(n n), u l(n n), w z 9(n n), w z \cdot 10(n n)$

$1=2$

1 cont inue

if (time.le.tb(1)) go to 2

$1=1+1$

if (1.gt. I max ) return

go to 1

2 cont inue

dtime $=t$ ime $-t b(1-1)$

if (itbd) $3,5,7$

3 cont inue

$v b=v b 1(1) * d t 1 m e+v b 2(1)$

if (Ip.eq. 1) vb $=\operatorname{dexp}($ omvb $(1) * d t 1 m e) * v b+v b 3(1)$

do 4 i $=1(n n-n i m i)$ ni

$u(j)=v b$

$u v(j)=v b$

4 cont inue

go to 100

5 cont inue

pnb $=$ pnb1(1)*dtime + pnb2 (1)

if (ip.eq. 1) pnb $=\operatorname{dexp}($ omp $(1) * \operatorname{dt}$ ime $) * p n b+\operatorname{pnb} 3(1)$

do $6 j=1,(n n-n i m 1) \cdot n 1$

$6 p(j)=$ pnb

go to 100

7 cont inue 


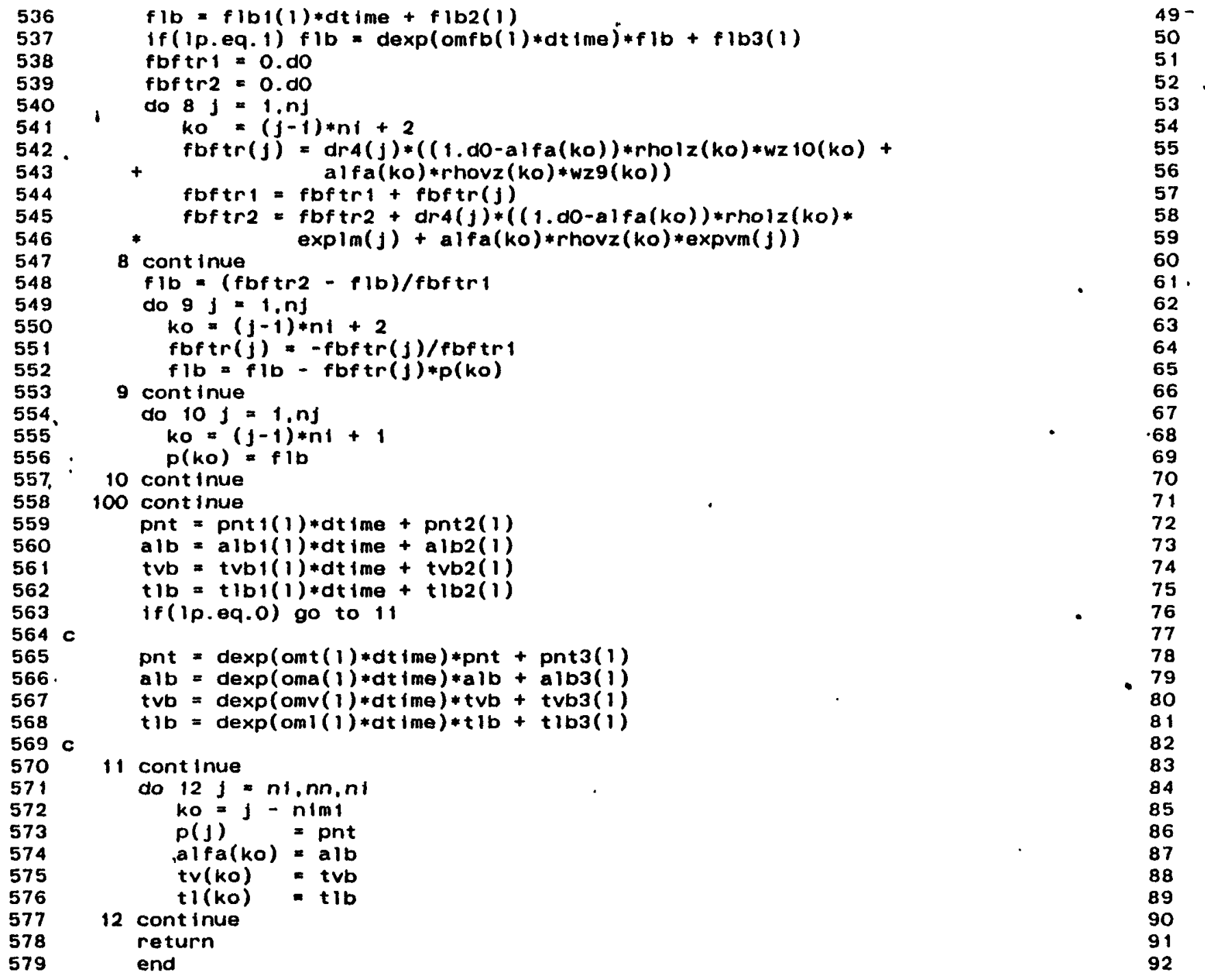




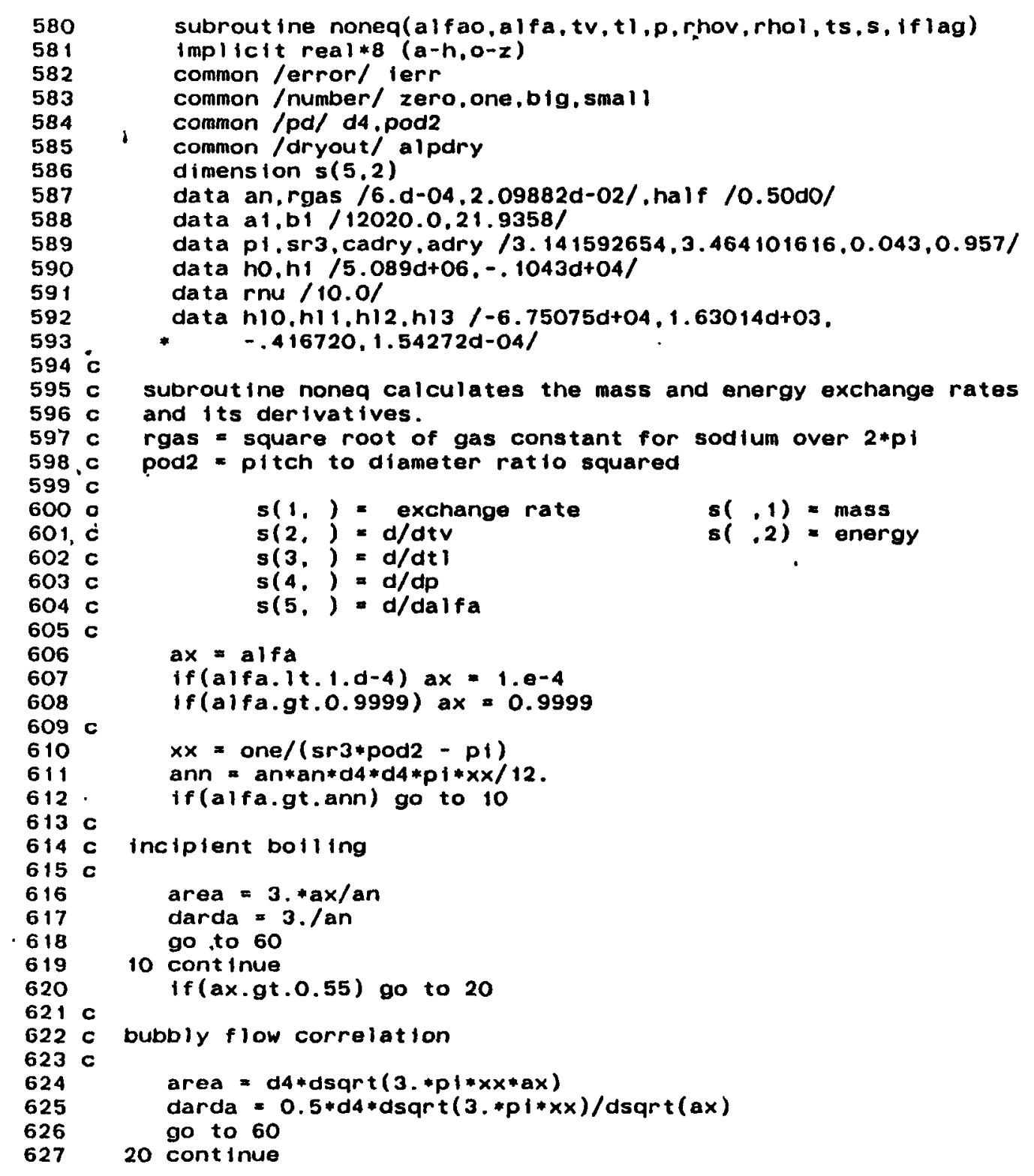




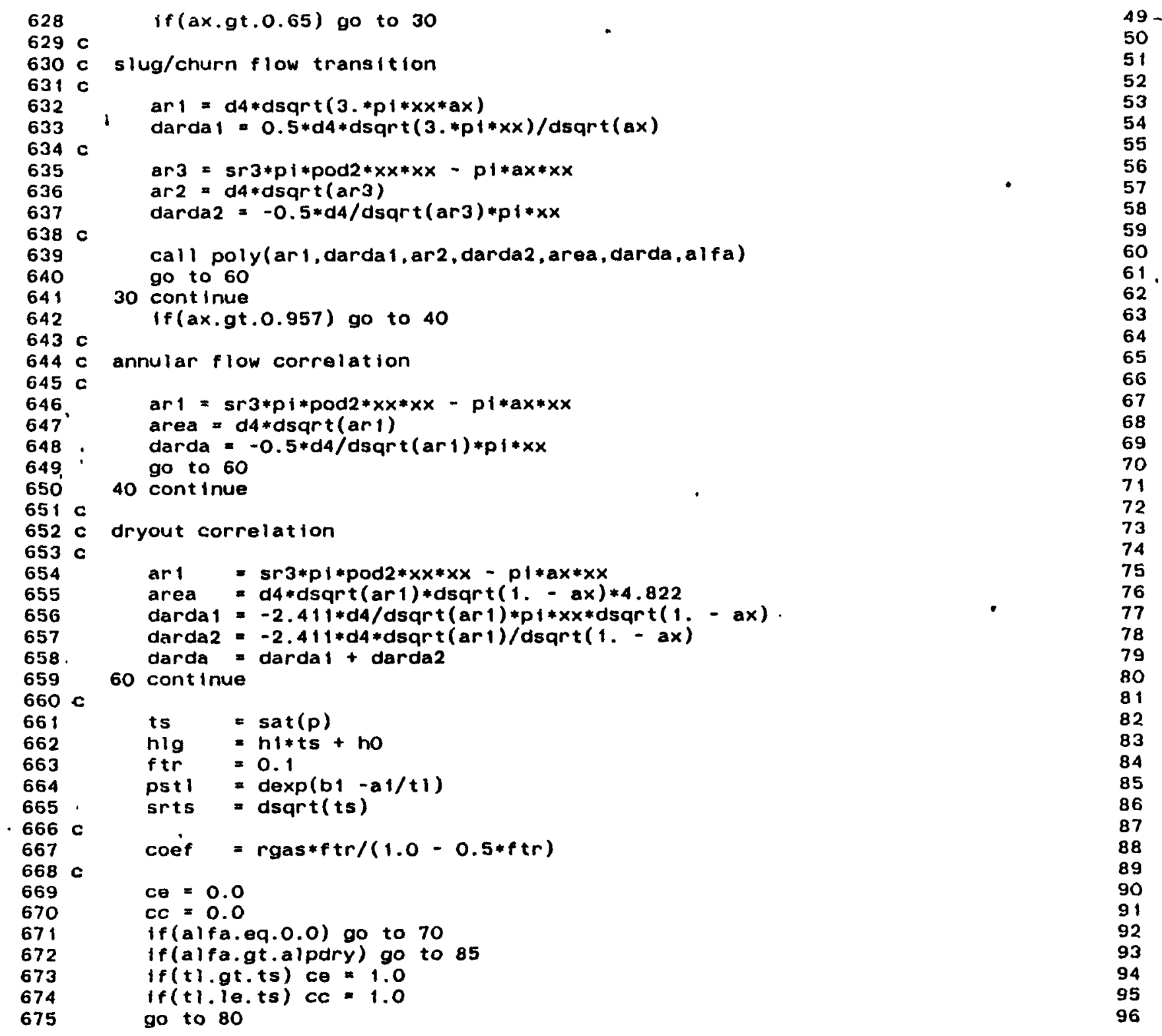




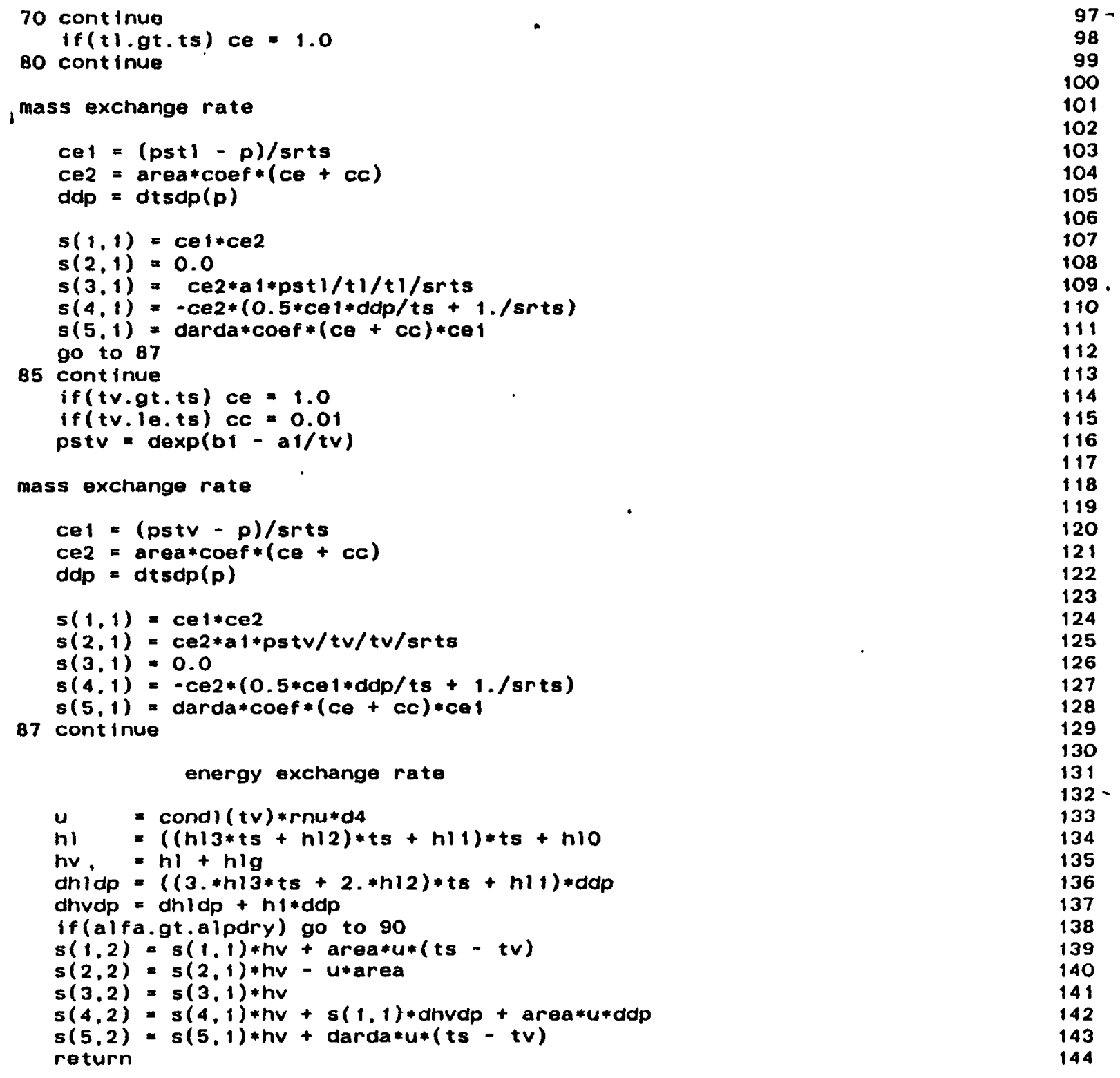

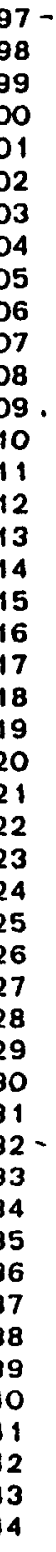


$s(2,2)=s(2,1) * h 1$

$s(3,2)=s(3,1) * h 1+$ area*u

$s(4,2)=s(4,1) * h 1+s(1,1) * d h l d p$ - area*u*ddp

$s(4,2)=s(4,1) *$ hi t s(1,1)*dhldp

$s(5,2)=s(5,1) * h 1+\operatorname{darda} * u *(t)-t s)$

return

end 
735 c this subroutine performs a polynomial fit for the area and the $736 \mathrm{c}$ the derivative of the area in the bubbly/annular flow transition

$737 \mathrm{c}$

738 


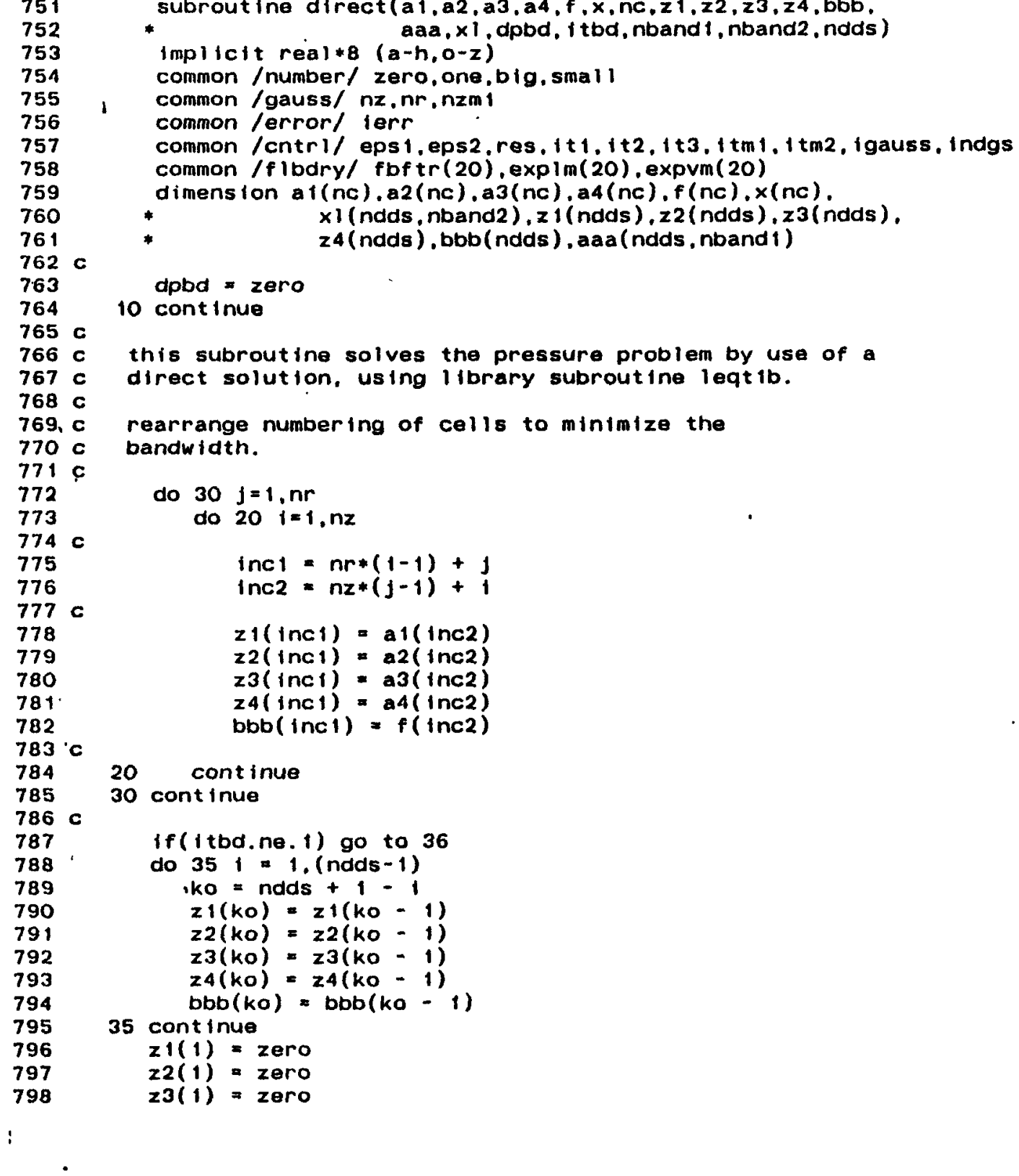




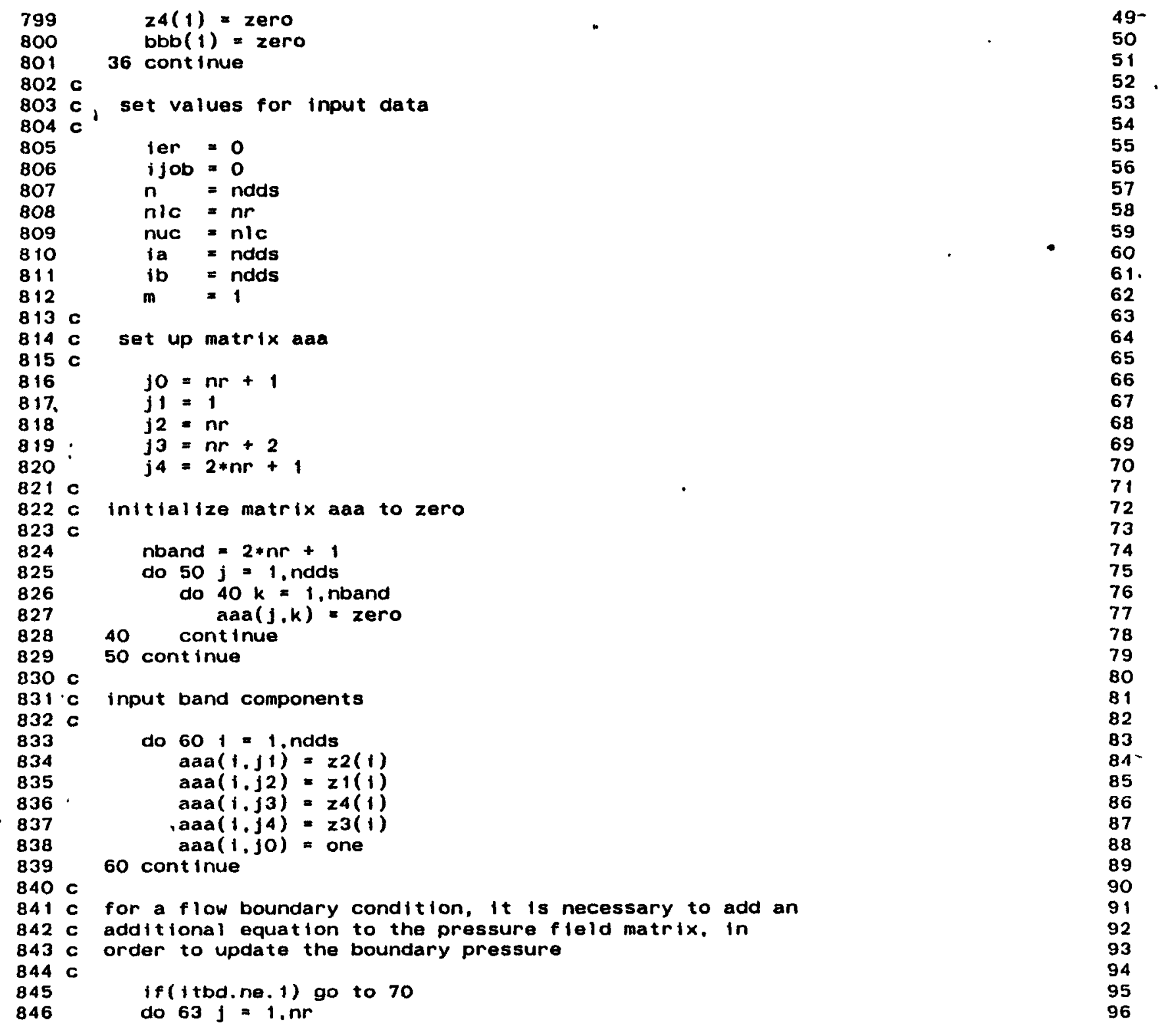




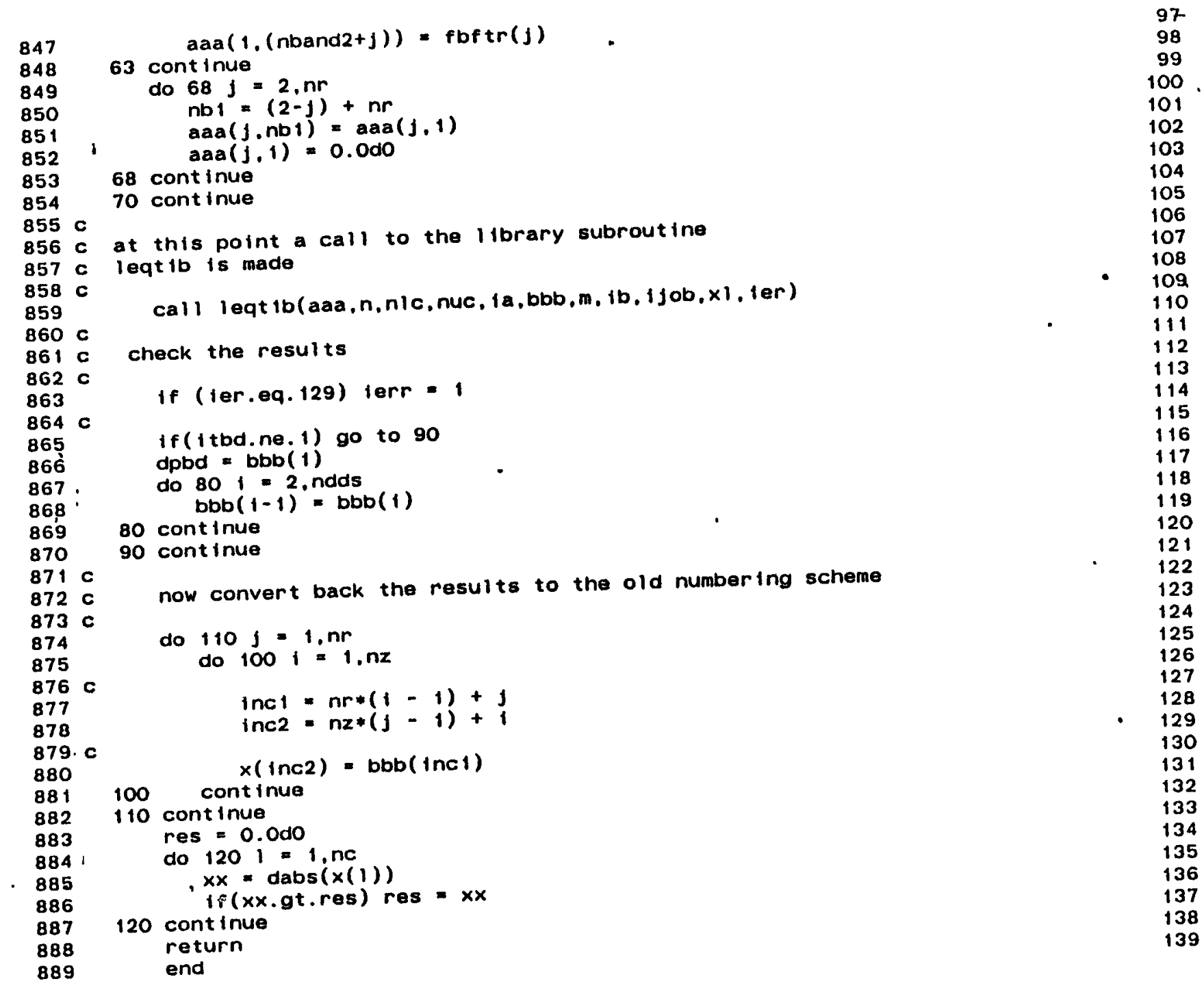

